\title{
Direct Formation of Oxocarbenium Ions under Weakly Acidic Conditions: Catalytic Enantioselective Oxa-Pictet-Spengler Reactions
}

\author{
Chenfei Zhao, Shawn B. Chen and Daniel Seidel* \\ Department of Chemistry and Chemical Biology, Rutgers, The State University of New Jersey, \\ Piscataway, New Jersey 08854, United States
}

\section{Supporting Information}

\section{Table of Contents}

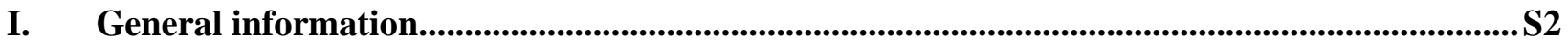

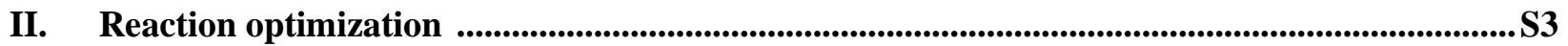

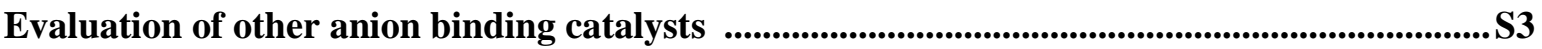

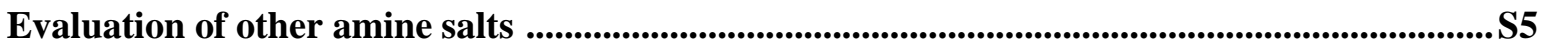

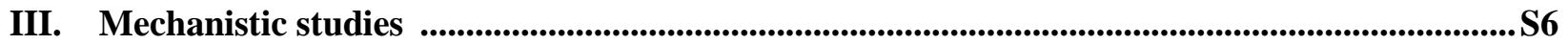

Evaluation of pyridinium salts with different $\mathrm{pKa}$ values ..........................................................6

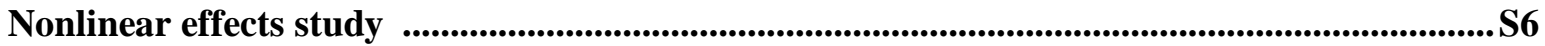

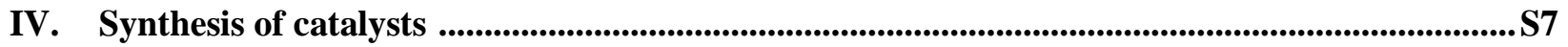

V. Preparation and characterization data of products .................................................................

VI. HPLC profiles of products ..........................................................................................................................S17

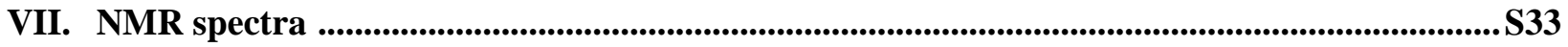

VIII. References ..............................................................................................................................................................S93 


\section{General information:}

Reagents and solvents were purchased from commercial sources and were purified by distillation or recrystallization prior to use. Toluene was freshly distilled from sodium under nitrogen prior to use. Reactions were run under a nitrogen atmosphere. Purification of reaction products was carried out by flash column chromatography using EM Reagent silica gel 60 (230-400 mesh). Analytical thin layer chromatography was performed on EM Reagent $0.25 \mathrm{~mm}$ silica gel $60 \mathrm{~F}_{254}$ plates. Visualization was accomplished with UV light, and potassium permanganate or Dragendorff-Munier stains, followed by heating. Melting points were recorded on a Thomas Hoover capillary melting point apparatus and are uncorrected. Infrared spectra were recorded on an ATI Mattson Genesis Series FT-Infrared spectrophotometer. Proton nuclear magnetic resonance spectra $\left({ }^{1} \mathrm{H}-\mathrm{NMR}\right)$ were recorded on a Varian VNMRS-500 MHz instrument and are reported in ppm using solvent as an internal standard $\left(\mathrm{CDCl}_{3}\right.$ at $7.26 \mathrm{ppm},\left(\mathrm{CD}_{3}\right)_{2} \mathrm{SO}$ at $2.50 \mathrm{ppm}, \mathrm{CD}_{3} \mathrm{OD}$ at $\left.3.31 \mathrm{ppm}\right)$. Data are reported as app = apparent, $\mathrm{s}=$ singlet, $\mathrm{d}=$ doublet, $\mathrm{t}=$ triplet, $\mathrm{dd}=$ doublet of doublets, $\mathrm{ddd}=$ doublet of doublet of doublets, dddd $=$ doublet of doublet of doublet of doublets, $\mathrm{m}=$ multiplet, comp = complex; integration; coupling constant(s) in Hz. Proton-decoupled carbon nuclear magnetic resonance spectra $\left({ }^{13} \mathrm{C}-\mathrm{NMR}\right)$ were recorded on a Varian VNMRS-500 MHz instrument and are reported in ppm using solvent as an internal standard $\left(\mathrm{CDCl}_{3}\right.$ at $77.16 \mathrm{ppm},\left(\mathrm{CD}_{3}\right)_{2} \mathrm{SO}$ at $39.52 \mathrm{ppm}, \mathrm{CD}_{3} \mathrm{OD}$ at $\left.49.00 \mathrm{ppm}\right)$. Mass spectra were recorded on a Finnigan LCQ-DUO mass spectrometer. HPLC analysis was carried out on an Agilent 1100 series instrument with auto sampler and multiple wavelength detectors. Optical rotations were measured using a $1 \mathrm{~mL}$ cell with a $1 \mathrm{dm}$ path length on a Jasco P-2000 polarimeter at $589 \mathrm{~nm}$ and at $20{ }^{\circ} \mathrm{C}$. Compounds $\mathbf{1 c},{ }^{1} \mathbf{1 d},{ }^{2} \mathbf{1 i},{ }^{3} \mathbf{2 a},{ }^{4}$ $\mathbf{2 b},{ }^{5} \mathbf{2 c},{ }^{6} \mathbf{2 d},{ }^{7} \mathbf{2} \mathbf{e}^{8}$ were prepared according to reported procedures. 


\section{Reaction optimization}

\section{Evaluation of other anion binding catalysts}
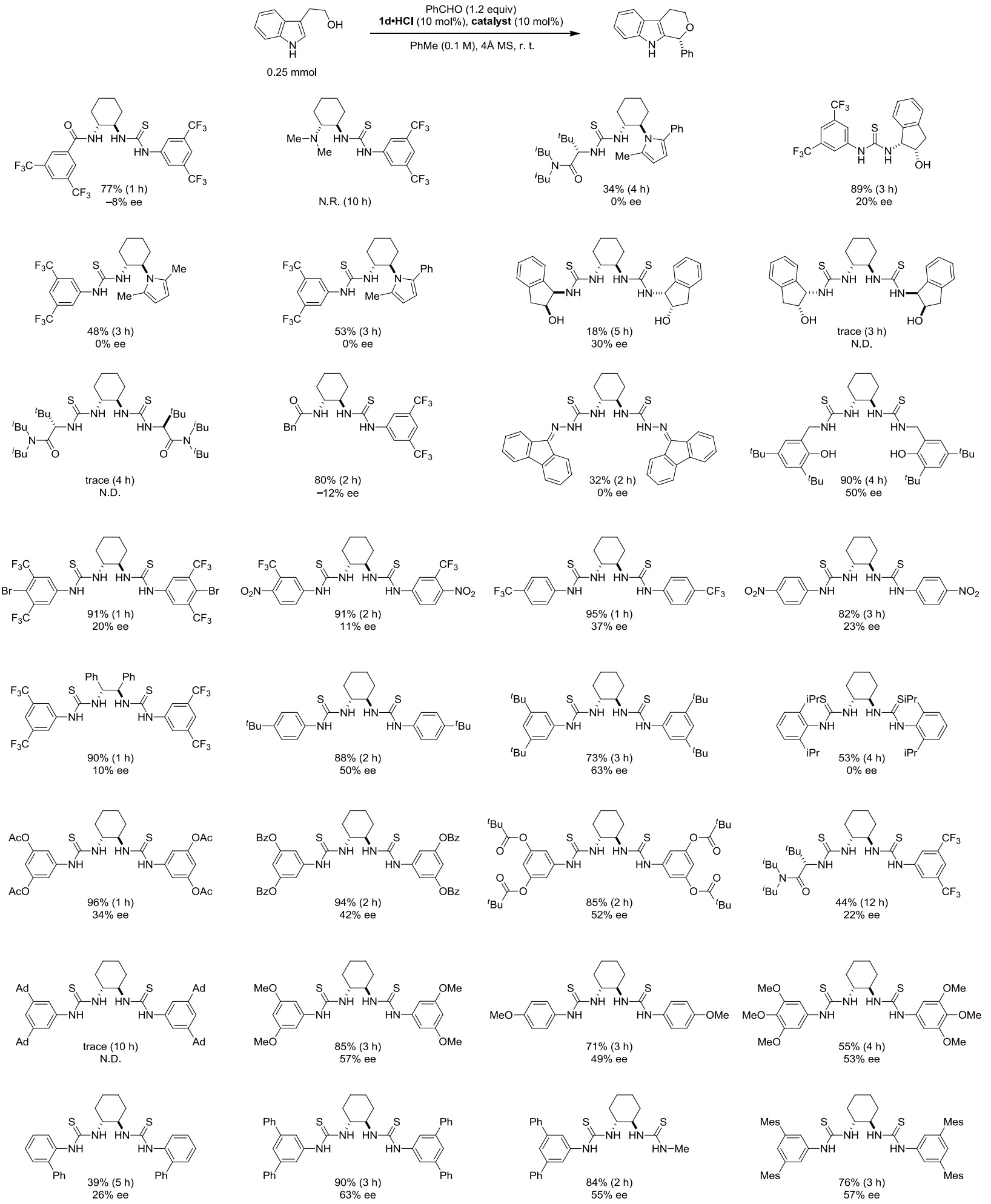

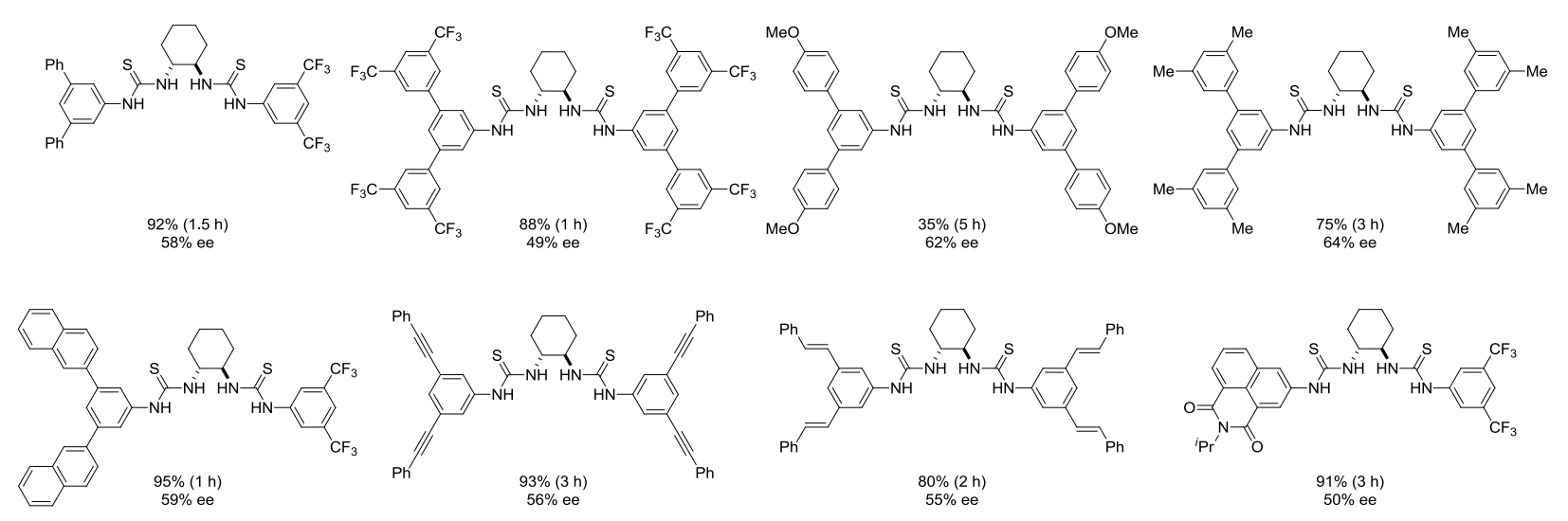

(1)
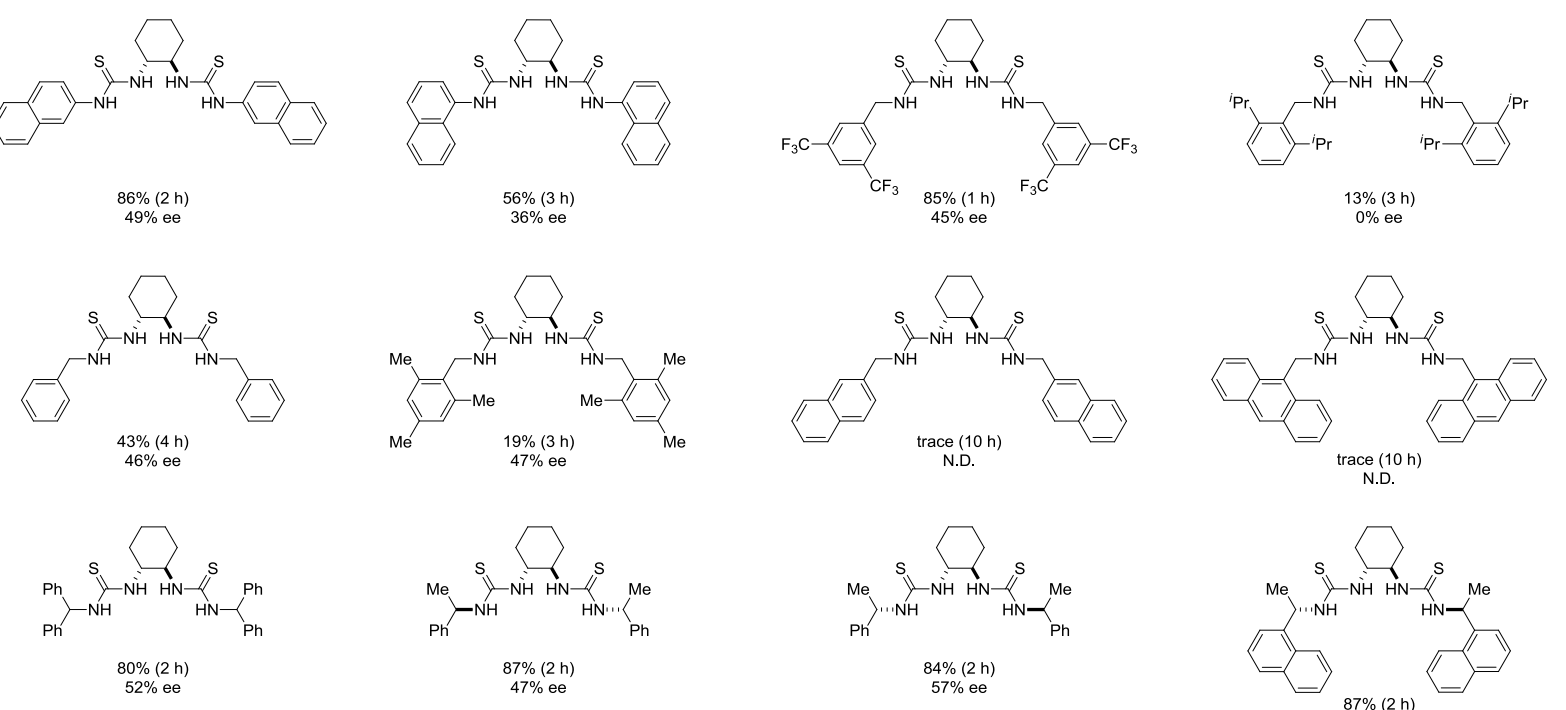

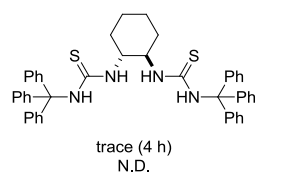
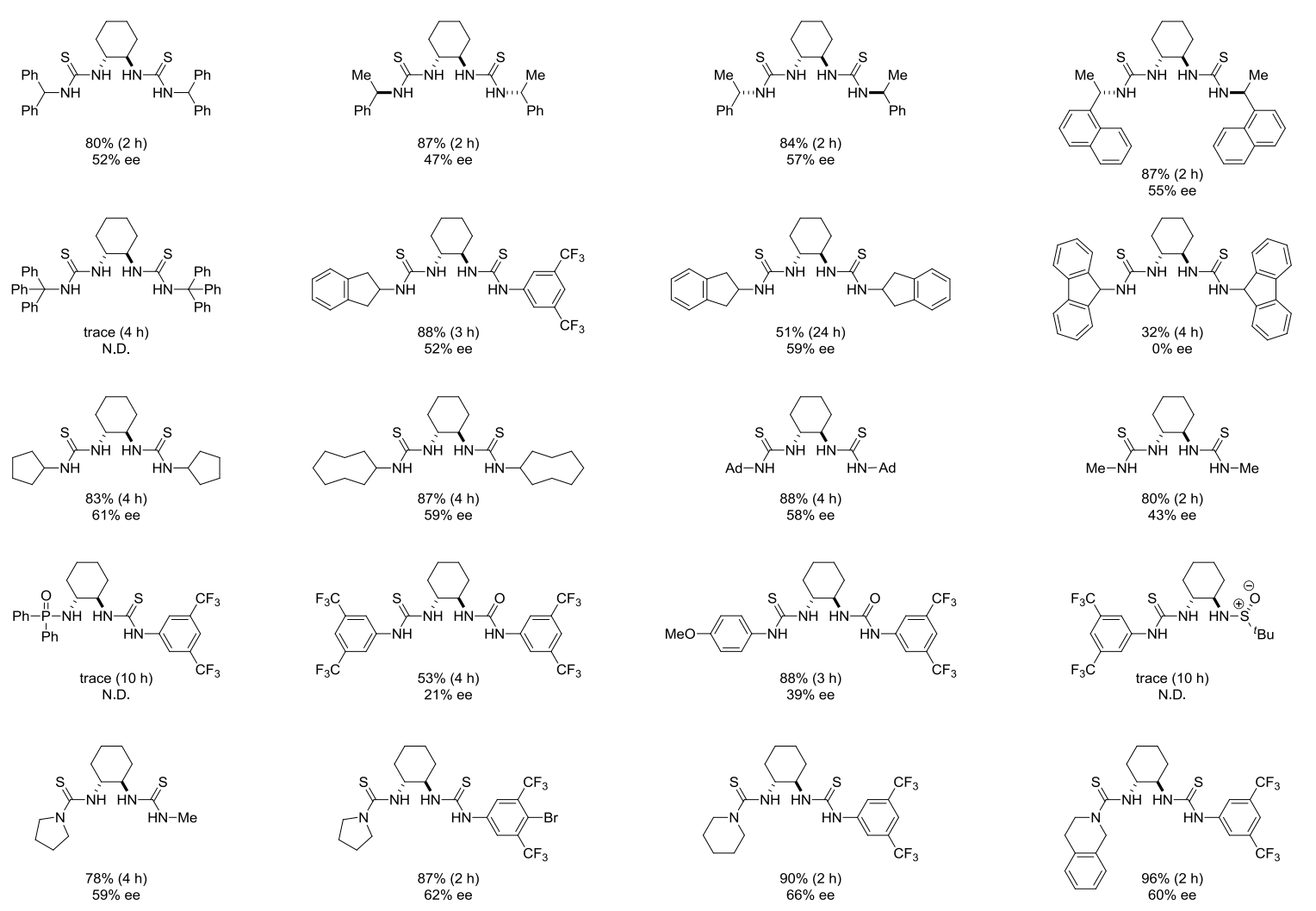

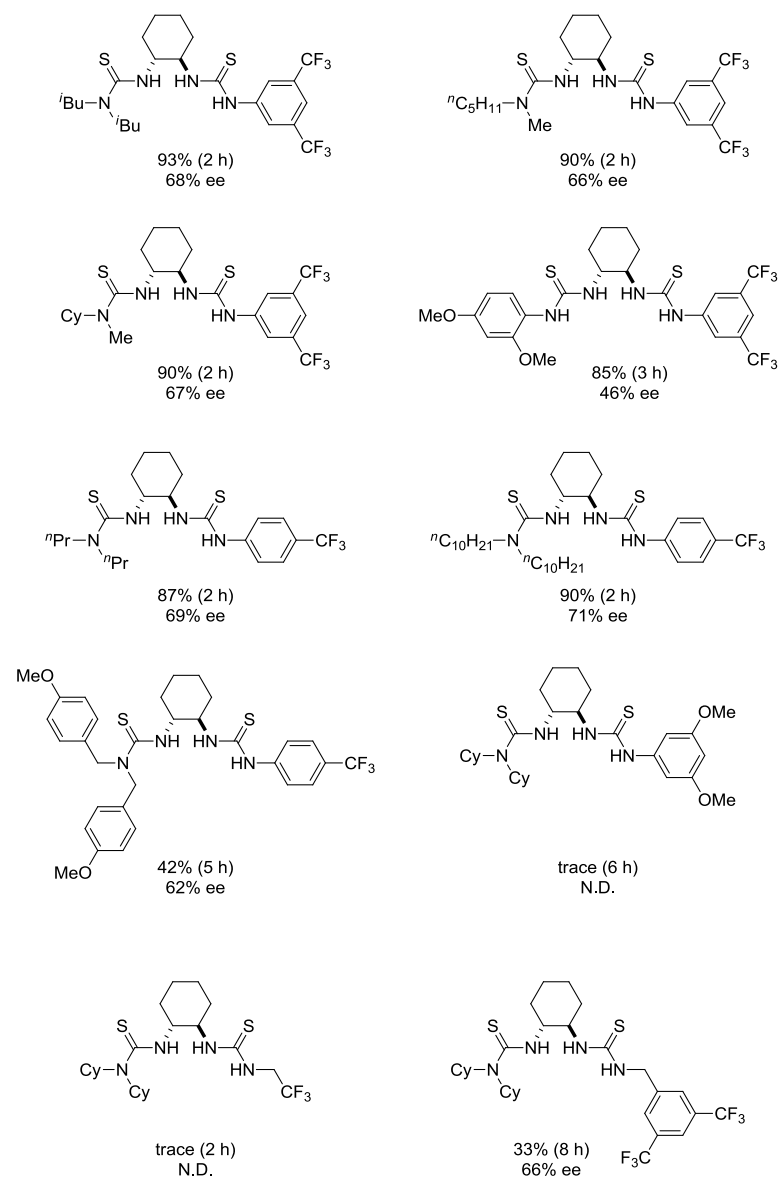
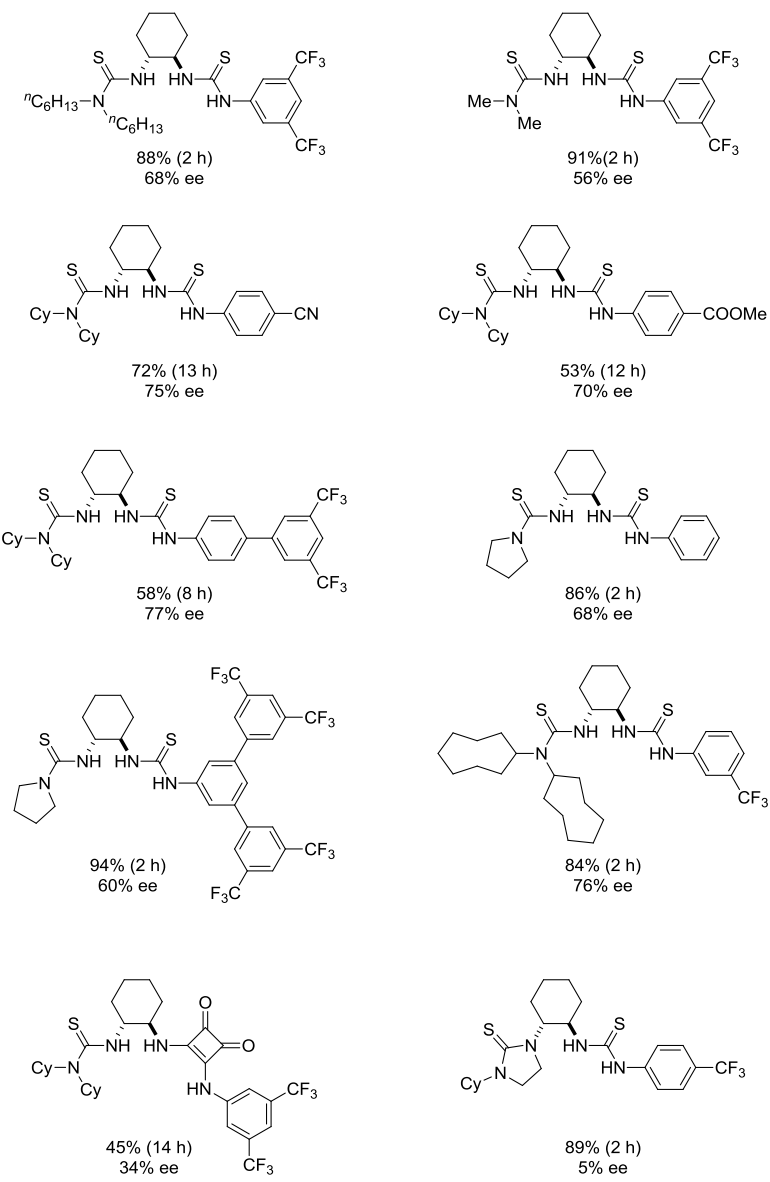

\section{Evaluation of other amine salts}

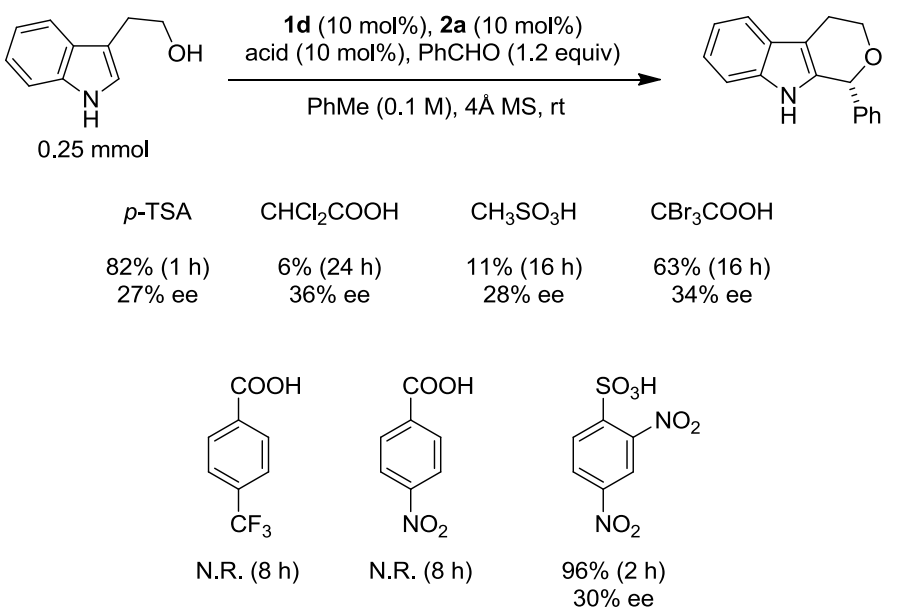




\section{Mechanistic studies}

\section{Evaluation of pyridinium salts with different $p K_{\mathrm{a}}$ values $^{9-12}$}

To a flame dried vial was added the pyridine derivative $(0.0275 \mathrm{mmol}, 11 \mathrm{~mol} \%)$, thiourea catalyst $2 \mathrm{a}$ (16 mg, $0.025 \mathrm{mmol}, 10 \mathrm{~mol} \%$ ), tryptophol (40 mg, $0.25 \mathrm{mmol}, 1$ equiv), $4 \AA \mathrm{MS}$ (100 mg) and dry toluene $(2.5 \mathrm{~mL}, 0.1 \mathrm{M})$. The resulting mixture was stirred under nitrogen and $\mathrm{HCl}(10 \mathrm{~mol} \%, 3.5 \mathrm{M}$ in dioxane) was added followed by benzaldehyde ( $30 \mu \mathrm{L}, 0.3 \mathrm{mmol}, 1.2$ equiv). The reaction was stirred for 24 hours before being quenched with triethylamine $(40 \mu \mathrm{L})$. The resulting mixture was directly purified by flash chromatography on silica gel topped with Celite.

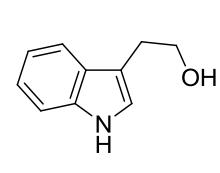

$0.25 \mathrm{mmol}$
0.7<smiles>Cc1ccccn1</smiles>

$45 \%$

$$
2.2
$$<smiles>N#Cc1ccncc1</smiles>

$3 \%$
$25 \%$ ee 2a (10 mol\%), $\mathrm{PhCHO}$ (1.2 equiv) pyridine derivative (11 mol\%) $\mathrm{HCl}$ (10 mol\%, 3.5 $\mathrm{M}$ in dioxane)

PhMe (0.1 M), $4 \AA$ MS, r.t, 24 h

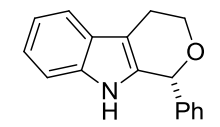

4.5

5.2

not reported<smiles>Clc1cccnc1</smiles>

$2 \%$
$27 \%$ ee
3.3<smiles>COc1ccccn1</smiles>

trace
N.D.<smiles>c1ccc(-c2ccccn2)cc1</smiles>

N.R.<smiles>c1ccncc1</smiles><smiles>COC(=O)c1ccccn1</smiles>

N.R.
$8 \%$

\section{Nonlinear effects study}

Following general procedure $\mathbf{C}$ for the enantioselective oxa-Pictet-Spengler reaction (see below), the reaction was set up using catalyst $\mathbf{2 l}$ with $0 \%, 20 \%, 40 \%, 60 \%, 80 \%,>99 \%$ ee at room temperature.

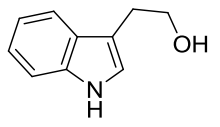

$0.25 \mathrm{mmol}$

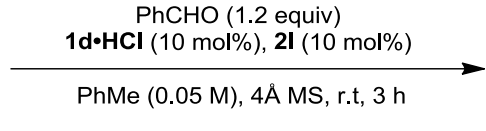

PhMe (0.05 M), 4A MS, r.t, 3 h

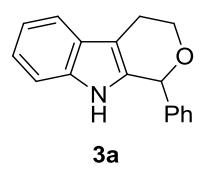

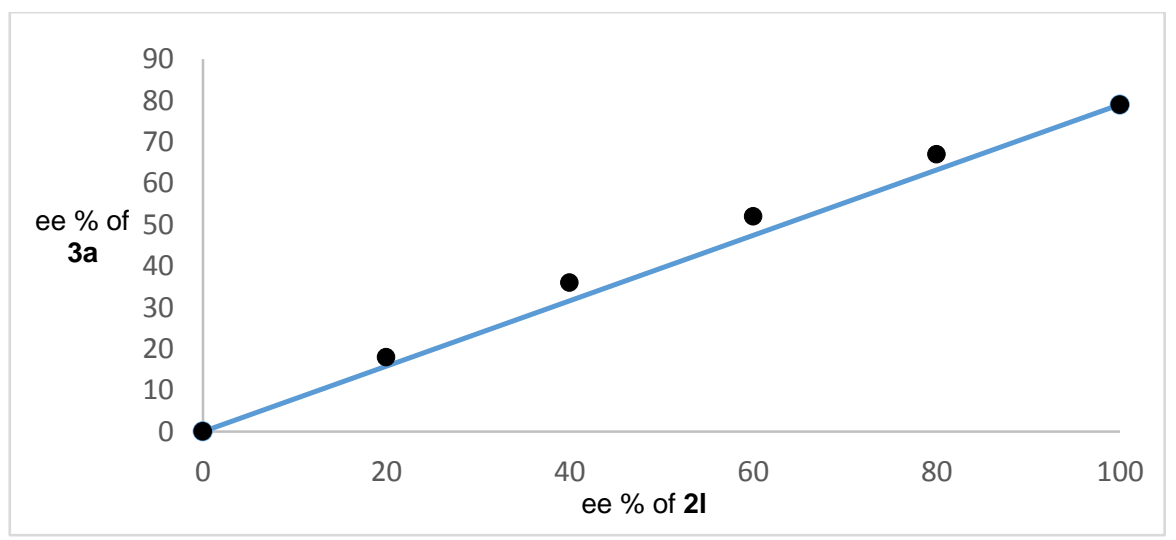




\section{Synthesis of catalysts}

\section{General procedure A for the synthesis of amine salts}

To a solution of the corresponding amine $(5.33 \mathrm{mmol}, 1$ equiv) in diethyl ether $(10.7 \mathrm{~mL}, 0.5 \mathrm{M})$ was added $\mathrm{HCl}$ ( $4 \mathrm{M}$ in dioxane, $1.33 \mathrm{~mL}, 5.33 \mathrm{mmol}, 1$ equiv) and the resulting mixture was stirred vigorously for $10 \mathrm{~min}$. After filtration, the solid was washed with cold $\left(\sim 0{ }^{\circ} \mathrm{C}\right)$ diethyl ether $(3 \times 5 \mathrm{~mL})$ and dried under vacuum to afford the amine salt.

(S)-2-(methoxycarbonyl)indolin-1-ium chloride $(\mathbf{1 d} \cdot \mathbf{H C l})$ : Following general procedure $\mathbf{A}, \mathbf{1 d} \cdot \mathbf{H C l}$

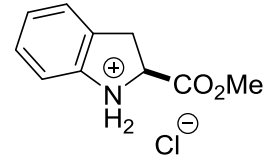
was obtained as a white solid in $95 \%$ yield; $\mathrm{mp}=161-163{ }^{\circ} \mathrm{C} ;[\alpha]_{\mathrm{D}}{ }^{20}-73.2(\mathrm{c} 0.5$, $\mathrm{CHCl}_{3}$ ); IR (KBr) 3119, 3054, 2957, 2359, 1741, 1506, 1391, 1236, 1143, 1021, 860, $758,420 \mathrm{~cm}^{-1}$; ${ }^{1} \mathrm{H}$ NMR $\left(500 \mathrm{MHz},\left(\mathrm{CD}_{3}\right)_{2} \mathrm{SO}\right) \delta 10.61$ (br s, 3H, contains water), 7.28 (app d, $J=7.4 \mathrm{~Hz}, 1 \mathrm{H}), 7.22$ (app td, $J=7.8,1.2 \mathrm{~Hz}, 1 \mathrm{H}), 7.17-7.05$ (comp, 2H), $4.78(\mathrm{dd}, J=9.8,6.9 \mathrm{~Hz}, 1 \mathrm{H}), 3.73(\mathrm{~s}, 3 \mathrm{H}), 3.44(\mathrm{dd}, J=16.2,9.8 \mathrm{~Hz}, 1 \mathrm{H}), 3.28(\mathrm{dd}, J=16.2,6.9 \mathrm{~Hz}$, $1 \mathrm{H}) ;{ }^{13} \mathrm{C}$ NMR $\left(125 \mathrm{MHz},\left(\mathrm{CD}_{3}\right)_{2} \mathrm{SO}\right) \delta 170.56,141.63,131.11,127.96,125.25,125.02,115.68,58.98$, $52.78,32.73 ; \mathrm{m} / z(\mathrm{ESI}-\mathrm{MS}) 178.1[\mathrm{M}-\mathrm{Cl}]^{+}$.

(2S)-2-(methoxycarbonyl)-1-methylindolin-1-ium chloride $(\mathbf{1 i} \cdot \mathbf{H C l})$ : Following general procedure $\mathbf{A}$,

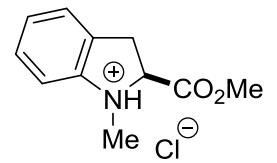

1i·HCl was obtained as a white solid in $70 \%$ yield; $m p=101-103{ }^{\circ} \mathrm{C} ;[\alpha]_{\mathrm{D}}{ }^{20}-58.3(\mathrm{c}$ $0.5, \mathrm{MeOH})$; IR (KBr) 3400, 2904, 1738, 1486, 1428, 1368, 1343, 1251, 1128, 995 , $825,803,763,605,513,428 \mathrm{~cm}^{-1} ;{ }^{1} \mathrm{H}$ NMR $\left(500 \mathrm{MHz},\left(\mathrm{CD}_{3}\right)_{2} \mathrm{SO}\right) \delta 9.83$ (br s, $2 \mathrm{H}$, contains water), 7.07-6.93 (comp, 2H), 6.60 (app td, $J=7.4,1.0 \mathrm{~Hz}, 1 \mathrm{H}), 6.49$ (app d, $J=7.8 \mathrm{~Hz}, 1 \mathrm{H}), 4.14(\mathrm{dd}, J=10.1,8.4 \mathrm{~Hz}, 1 \mathrm{H}), 3.69(\mathrm{~s}, 3 \mathrm{H}), 3.30(\mathrm{dd}, J=16.0,10.2 \mathrm{~Hz}, 1 \mathrm{H}), 2.98(\mathrm{dd}$, $J=15.9,8.4 \mathrm{~Hz}, 1 \mathrm{H}), 2.75(\mathrm{~s}, 3 \mathrm{H}) ;{ }^{13} \mathrm{C}$ NMR $\left(125 \mathrm{MHz},\left(\mathrm{CD}_{3}\right)_{2} \mathrm{SO}\right) \delta 172.52,151.98,127.57,126.89$, $123.89,117.67,106.67,66.72,51.90,34.39,32.75 ; \mathrm{m} / \mathrm{z}(\mathrm{ESI}-\mathrm{MS}) 192.1[\mathrm{M}-\mathrm{Cl}]^{+}$.

(S)-2-(methoxycarbonyl)indolin-1-ium bromide (1d·HBr): Following general procedure A, except for

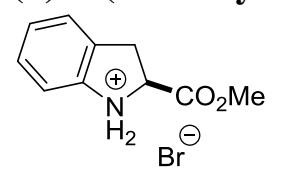
using $\mathrm{HBr}(48 \%$ in water), $\mathbf{1 d} \cdot \mathbf{H B r}$ was obtained as a white solid in $77 \%$ yield; $\mathrm{mp}=$ $172-174{ }^{\circ} \mathrm{C} ;[\alpha]_{\mathrm{D}}{ }^{20}-50.7(\mathrm{c} 0.5, \mathrm{MeOH}) ; \mathrm{IR}(\mathrm{KBr}) 3092,3054,2997,2954,2587$, $2514,1738,1503,1378,1348,1231,1020,860,753 \mathrm{~cm}^{-1}$; ${ }^{1} \mathrm{H}$ NMR $(500 \mathrm{MHz}$, $\left.\mathrm{CD}_{3} \mathrm{OD}\right) 7.54-7.49$ (comp, 3H), 7.49-7.44 (m, 1H), $5.13(\mathrm{dd}, J=9.5,7.3 \mathrm{~Hz}, 1 \mathrm{H})$, $3.91(\mathrm{~s}, 3 \mathrm{H}), 3.73(\mathrm{dd}, J=16.5,9.5 \mathrm{~Hz}, 1 \mathrm{H}), 3.54(\mathrm{dd}, J=16.3,7.3 \mathrm{~Hz}, 1 \mathrm{H}) ;{ }^{13} \mathrm{C}$ NMR $(125 \mathrm{MHz}$, $\left.\mathrm{CD}_{3} \mathrm{OD}\right) \delta 169.91,136.81,134.85,131.27,130.04,127.32,120.19,61.28,54.18,33.93 ; \mathrm{m} / \mathrm{z}(\mathrm{ESI}-\mathrm{MS})$ $178.2[\mathrm{M}-\mathrm{Br}]^{+}$.

(S)-2-(methoxycarbonyl)indolin-1-ium iodide $(\mathbf{1 d} \cdot \mathbf{H I})$ : Following general procedure A, except for

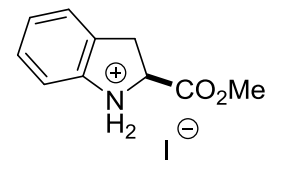
using HI (57\% in water), 1d·HI was obtained as a yellow solid in $87 \%$ yield; $\mathrm{mp}=$ $145-147{ }^{\circ} \mathrm{C} ; \quad[\alpha]_{\mathrm{D}}{ }^{20}-39.8(\mathrm{c} 0.5, \mathrm{MeOH}) ; \mathrm{IR}(\mathrm{KBr}) 3417,3379,2942,2914,2464$, $1756,1428,1351,1283,1246,1208,1161,1001,763 \mathrm{~cm}^{-1}$; ${ }^{1} \mathrm{H}$ NMR $(500 \mathrm{MHz}$, $\left.\left(\mathrm{CD}_{3}\right)_{2} \mathrm{SO}\right) \delta 7.14(\operatorname{app~d}, J=7.2 \mathrm{~Hz}, 1 \mathrm{H}), 7.07(\operatorname{app~td}, J=7.8,1.1 \mathrm{~Hz}, 1 \mathrm{H}), 6.86-$ 6.78 (comp, 2H), 6.50 (br s, $4 \mathrm{H}$, contains water), $4.60(\mathrm{dd}, J=10.2,6.4 \mathrm{~Hz}, 1 \mathrm{H}), 3.70$ (s, 3H), 3.37 (dd, $J$ $=16.2,10.2 \mathrm{~Hz}, 1 \mathrm{H}), 3.17(\mathrm{dd}, J=16.2,6.2 \mathrm{~Hz}, 1 \mathrm{H}) ;{ }^{13} \mathrm{C} \mathrm{NMR}\left(125 \mathrm{MHz},\left(\mathrm{CD}_{3}\right)_{2} \mathrm{SO}\right) \delta 172.51,146.54$, $128.44,127.62,124.66,121.03,111.73,59.18,52.34,32.99 ; \mathrm{m} / z(\mathrm{ESI}-\mathrm{MS}) 178.4[\mathrm{M}-\mathrm{I}]^{+}$. 
General procedure B for the synthesis of thiourea catalysts

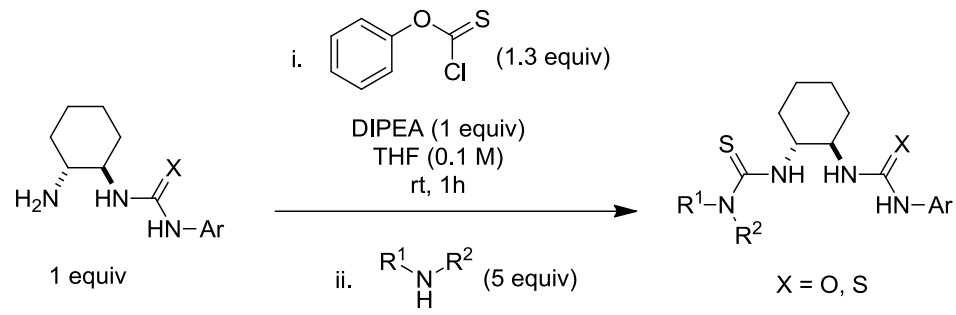

To a solution of amino(thio) urea $^{13,14}(0.39 \mathrm{mmol}, 1$ equiv) in dry THF $(3.9 \mathrm{~mL}, 0.1 \mathrm{M})$ was added $o$ phenyl chlorothionoformate $(70 \mu \mathrm{L}, 0.51 \mathrm{mmol}, 1.3$ equiv) and $N, N$-diisopropylethylamine $(68 \mu \mathrm{L}, 0.39$ mmol, 1 equiv). The resulting solution was stirred at room temperature for $1 \mathrm{~h}$, followed by addition of the amine ( $1.95 \mathrm{mmol}, 5$ equiv). The resulting mixture was stirred at room temperature for the indicated time. Subsequently, saturated aqueous $\mathrm{NaHCO}_{3}(10 \mathrm{~mL})$ was added and the mixture was extracted with EtOAc $(3 \times 10 \mathrm{~mL})$. The combined organic layers were washed with brine $(10 \mathrm{~mL})$, dried over $\mathrm{Na}_{2} \mathrm{SO}_{4}$ and filtered. The resulting solution was then concentrated under reduced pressure and purified by flash chromatography on silica gel.

\section{$N$-((1R,2R)-2-(3-(3,5-bis(trifluoromethyl)phenyl)thioureido)cyclohexyl)pyrrolidine-1-}

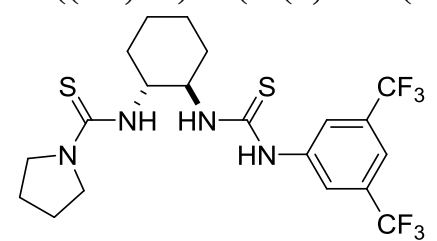

carbothioamide (2f): Following general procedure $\mathbf{B}$, the reaction was stirred at room temperature for 2 hours and $\mathbf{2 f}$ was isolated as a white solid in $88 \%$ yield; $m p=171-173{ }^{\circ} \mathrm{C} ; \quad \mathrm{R}_{\mathrm{f}}=0.30($ Hexanes $/$ EtOAc $70: 30 \mathrm{v} / \mathrm{v})$; $[\alpha]_{\mathrm{D}}^{20}+68.2\left(\mathrm{c} 0.5, \mathrm{CHCl}_{3}\right) ; \mathrm{IR}(\mathrm{KBr}) 3246,3048,2938,2861,1545,1385$, 1277, 1178, 1132, 971, 885, $681 \mathrm{~cm}^{-1} ;{ }^{1} \mathrm{H}$ NMR (500 MHz, $\left.\mathrm{CDCl}_{3}\right) \delta 9.56$ $(\mathrm{s}, 1 \mathrm{H}), 8.37(\mathrm{~d}, J=8.3 \mathrm{~Hz}, 1 \mathrm{H}), 8.25(\mathrm{~s}, 2 \mathrm{H}), 7.57(\mathrm{~s}, 1 \mathrm{H}), 7.09(\mathrm{~d}, J=5.9$ $\mathrm{Hz}, 1 \mathrm{H}), 4.73-4.60$ (m, 1H), 4.07-3.82 (comp, 2H), 3.65-3.42 (comp, 2H), 3.42-3.25 (m, 1H), 2.72-2.58 (m, 1H), 2.28-2.16 (m, 1H), 2.13-1.72 (comp, 6H), 1.73-1.61 (m, 1H), 1.51-1.37 (m, 1H), 1.37-1.17 (comp, 2H); ${ }^{13} \mathrm{C}$ NMR $\left(125 \mathrm{MHz}, \mathrm{CDCl}_{3}\right) \delta 181.42,175.90,140.77,131.79\left(\mathrm{q}, J_{C-F}=33.5 \mathrm{~Hz}\right), 123.23(\mathrm{q}$, $\left.J_{C-F}=272.8 \mathrm{~Hz}\right), 122.25,117.62,62.38,56.43,52.58,48.57,32.77,32.74,26.03,25.07,24.77,24.54$; $\mathrm{m} / z$ (ESI-MS) $497.1[\mathrm{M}-\mathrm{H}]^{-}$.

\section{$N$-((1R,2R)-2-(3-(3,5-bis(trifluoromethyl)phenyl)ureido)cyclohexyl)pyrrolidine-1-carbothioamide}

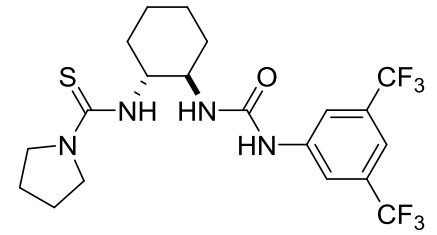

(2g): Following general procedure $\mathbf{B}$, the reaction was stirred at room temperature for 2 hours and $\mathbf{2 g}$ was isolated as a white solid in $66 \%$ yield; $\mathrm{mp}>200{ }^{\circ} \mathrm{C} ; \mathrm{R}_{\mathrm{f}}=0.28($ Hexanes$/$ EtOAc $70: 30 \mathrm{v} / \mathrm{v}) ;[\alpha]_{\mathrm{D}}{ }^{20}+31.9(\mathrm{c} 0.5$, $\mathrm{CHCl}_{3}$ ); IR (KBr) 3349, 3307, 3282, 3207, 3087, 2938, 1697, 1571, 1474, $1391,1275,1186,1130,1023,895,870,703,673 \mathrm{~cm}^{-1} ;{ }^{1} \mathrm{H}$ NMR $(500$ $\left.\mathrm{MHz}, \mathrm{CDCl}_{3}\right) \delta 8.56(\mathrm{~s}, 1 \mathrm{H}), 8.00(\mathrm{~s}, 2 \mathrm{H}), 7.41(\mathrm{~s}, 1 \mathrm{H}), 6.95(\mathrm{~d}, J=5.5 \mathrm{~Hz}$, $1 \mathrm{H}), 6.90(\mathrm{~d}, J=7.3 \mathrm{~Hz}, 1 \mathrm{H}), 4.14-3.92(\mathrm{~m}, 1 \mathrm{H}), 3.91-3.71$ (comp, 2H), 3.66-3.50 (m, 1H), 3.49-3.28 (comp, 2H), 2.69-2.58 (m, 1H), 2.21-2.14 (m, 1H), 2.14-1.96 (m, 1H), 1.95-1.75 (comp, 5H), 1.64-1.52 $(\mathrm{m}, 1 \mathrm{H}), 1.47-1.37(\mathrm{~m}, 1 \mathrm{H}), 1.37-1.24(\mathrm{~m}, 1 \mathrm{H}), 1.23-1.10(\mathrm{~m}, 1 \mathrm{H}) ;{ }^{13} \mathrm{C}$ NMR $\left(125 \mathrm{MHz}, \mathrm{CDCl}_{3}\right) \delta$ $176.22,156.72,141.52,132.25\left(\mathrm{q}, J_{C-F}=33.2 \mathrm{~Hz}\right), 123.39$ (q, $\left.J_{C-F}=272.7 \mathrm{~Hz}\right), 117.48,115.03,62.88$, 53.01, 52.36, 47.42, 32.74, 32.63, 25.77, 25.31, 24.79, 24.58; $\mathrm{m} / z$ (ESI-MS) $481.2[\mathrm{M}-\mathrm{H}]^{-}$. 


\section{$N$-((1R,2R)-2-(3-(3,5-bis(trifluoromethyl)phenyl)thioureido)cyclohexyl)pyrrolidine-1-carboxamide}

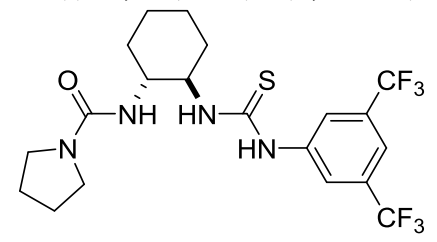

(2h): Following general procedure B, except for using phenyl chloroformate, the reaction was stirred at room temperature for 2 days and 2h was isolated as a white solid in $44 \%$ yield; $\mathrm{mp}=188-190{ }^{\circ} \mathrm{C} ; \mathrm{R}_{\mathrm{f}}=0.18$ (Hexanes/EtOAc 40:60 v/v); $[\alpha]_{\mathrm{D}}{ }^{20}+41.7\left(\mathrm{c} 0.5, \mathrm{CHCl}_{3}\right) ; \mathrm{IR}(\mathrm{KBr}) 3300$, 3097, 2938, 2859, 1618, 1535, 1474, 1396, 1278, 1181, 1131, 968, 880, 678 $\mathrm{cm}^{-1} ;{ }^{1} \mathrm{H}$ NMR $\left(500 \mathrm{MHz}, \mathrm{CDCl}_{3}\right) \delta 9.74(\mathrm{~s}, 1 \mathrm{H}), 8.21(\mathrm{~d}, J=9.1 \mathrm{~Hz}, 1 \mathrm{H})$, $7.97(\mathrm{~s}, 2 \mathrm{H}), 7.56(\mathrm{~s}, 1 \mathrm{H}), 5.00(\mathrm{~d}, J=8.8 \mathrm{~Hz}, 1 \mathrm{H}), 4.69-4.54(\mathrm{~m}, 1 \mathrm{H}), 3.68-3.58(\mathrm{~m}, 1 \mathrm{H}), 3.46-3.30$ (comp, 2H), 3.21-3.02 (comp, 2H), 2.28-2.18 (m, 1H), 2.14-2.05 (m, 1H), 1.98-1.67 (comp, 6H), 1.551.28 (comp, 4H); ${ }^{13} \mathrm{C}$ NMR $\left(125 \mathrm{MHz}, \mathrm{CDCl}_{3}\right) \delta 182.08,157.40,141.11,131.53\left(\mathrm{q}, J_{C-F}=33.4 \mathrm{~Hz}\right)$, $124.27,123.26\left(\mathrm{q}, J_{C-F}=271.3 \mathrm{~Hz}\right), 117.87,57.39,56.25,46.33,33.77,32.65,25.34,24.94 ; \mathrm{m} / z$ (ESIMS) $481.2[\mathrm{M}-\mathrm{H}]^{-}$.

$N$-((1R,2R)-2-(3-(3,5-bis(trifluoromethyl)phenyl)thioureido)cyclohexyl)piperidine-1-carbothioamide

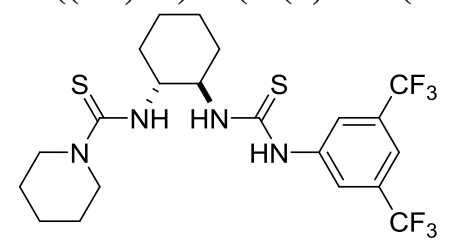

(2i): Following general procedure $\mathbf{B}$, the reaction was stirred at room temperature for 5 hours and $\mathbf{2 i}$ was isolated as a white solid in $69 \%$ yield; $\mathrm{mp}=96-98^{\circ} \mathrm{C} ; \mathrm{R}_{\mathrm{f}}=0.21\left(\right.$ Hexanes/EtOAc 80:20 v/v); $[\alpha]_{\mathrm{D}}{ }^{20}+93.1(\mathrm{c} 0.5$, $\mathrm{CHCl}_{3}$ ); IR (KBr) 3266, 3042, 2939, 2859, 1541, 1474, 1385, 1333, 1277, $1178,1132,968,885,681 \mathrm{~cm}^{-1}$; ${ }^{1} \mathrm{H}$ NMR $\left(500 \mathrm{MHz}, \mathrm{CDCl}_{3}\right) \delta 9.42(\mathrm{~s}$, $1 \mathrm{H}), 8.31(\mathrm{~d}, J=8.3 \mathrm{~Hz}, 1 \mathrm{H}), 8.18(\mathrm{~s}, 2 \mathrm{H}), 7.57(\mathrm{~s}, 1 \mathrm{H}), 7.33(\mathrm{~d}, J=5.8 \mathrm{~Hz}$, 1H), 4.71-4.59 (m, 1H), 4.18-4.06 (m, 1H), 3.94-3.78 (comp, 2H), 3.78-3.66 (comp, 2H), 2.69-2.57 (m, 1H), 2.27-2.17 (m, 1H), 1.97-1.79 (comp, 2H), 1.76-1.38 (comp, 8H), 1.38-1.18 (comp, 2H); ${ }^{13} \mathrm{C} \mathrm{NMR}$ $\left(125 \mathrm{MHz}, \mathrm{CDCl}_{3}\right) \delta 181.49,177.86,140.65,131.82\left(\mathrm{q}, J_{C-F}=33.5 \mathrm{~Hz}\right), 123.22\left(\mathrm{q}, J_{C-F}=272.9 \mathrm{~Hz}\right)$, $122.53,117.81,62.76,56.63,49.61,32.63,32.51,25.54,25.07,24.76,24.17 ; \mathrm{m} / z$ (ESI-MS) 511.1 [M $\mathrm{H}]^{-}$.

3-((1R,2R)-2-(3-(3,5-bis(trifluoromethyl)phenyl)thioureido)cyclohexyl)-1,1-dicyclohexylthiourea (2j):

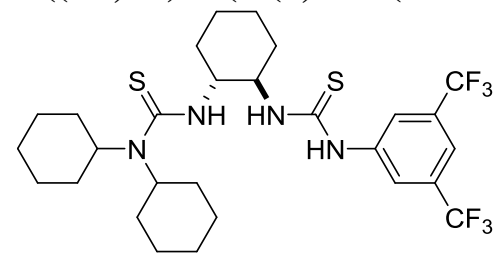
Following general procedure $\mathbf{B}$, the reaction was stirred at room temperature for 24 hours and $\mathbf{2} \mathbf{j}$ was isolated as a white solid in $66 \%$ yield; $\mathrm{mp}=98-100{ }^{\circ} \mathrm{C} ; \mathrm{R}_{\mathrm{f}}=0.36($ Hexanes $/$ EtOAc $90: 10 \mathrm{v} / \mathrm{v}) ;[\alpha]_{\mathrm{D}}{ }^{20}$ -27.1 (c $\left.0.5, \mathrm{CHCl}_{3}\right)$; IR (KBr) 3442, 3247, 2935, 2857, 1534, 1473, $1383,1323,1278,1178,1135,968,885,675 \mathrm{~cm}^{-1} ;{ }^{1} \mathrm{H}$ NMR $(500 \mathrm{MHz}$, $\left.\mathrm{CDCl}_{3}\right) \delta 8.40-8.17$ (comp, 2H), $7.91(\mathrm{~s}, 2 \mathrm{H}), 7.60(\mathrm{~s}, 1 \mathrm{H}), 5.61(\mathrm{~d}, J=$ $8.4 \mathrm{~Hz}, 1 \mathrm{H}), 4.72-4.58(\mathrm{~m}, 1 \mathrm{H}), 4.49-3.94$ (comp, 3H), 2.43-2.28 (m, 1H), 2.25-2.13 (m, 1H), 1.95-1.46 (comp, 16H), 1.46-1.15 (comp, 8H), 1.12-1.00 (comp, 2H); ${ }^{13} \mathrm{C}$ NMR $\left(125 \mathrm{MHz}, \mathrm{CDCl}_{3}\right) \delta 180.72$, $180.50,140.24,132.05\left(\mathrm{q}, J_{C-F}=36.6 \mathrm{~Hz}\right), 123.58,123.18\left(\mathrm{q}, J_{C-F}=272.7 \mathrm{~Hz}\right), 118.33,61.17,59.75$, $57.86,32.76,32.05,31.55,30.94,26.16,25.50,25.05,24.48 ; \mathrm{m} / z$ (ESI-MS) $607.1[\mathrm{M}-\mathrm{H}]^{-}$.

3-((1R,2R)-2-(3-(3,5-bis(trifluoromethyl)phenyl)thioureido)cyclohexyl)-1,1-dicyclooctylthiourea (2k):

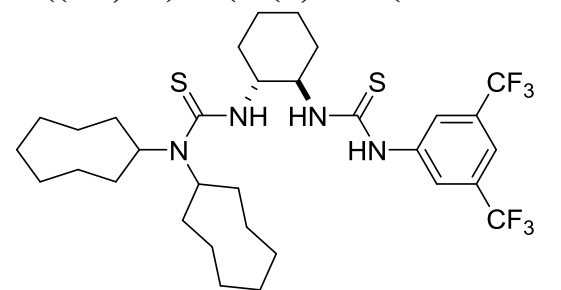
Following general procedure $\mathbf{B}$ (using 2.5 equivalents of dicyclooctylamine ${ }^{15}$ ), the reaction was stirred at room temperature for 2 days to afford $\mathbf{2 k}$ as a white solid in $61 \%$ yield; $\mathrm{mp}=105-108$ ${ }^{\circ} \mathrm{C} ; \quad \mathrm{R}_{\mathrm{f}}=0.33\left(\right.$ Hexanes/EtOAc 85:15 v/v); $[\alpha]_{\mathrm{D}}{ }^{20}-31.4$ (c 0.5 , $\mathrm{CHCl}_{3}$ ); IR (KBr) 3437, 3247, 2926, 2855, 1534, 1473, 1384, 1323, $1277,1178,1135,973,880,680 \mathrm{~cm}^{-1} ;{ }^{1} \mathrm{H}$ NMR (500 MHz, $\left.\mathrm{CDCl}_{3}\right)$ $\delta 8.44(\mathrm{~s}, 1 \mathrm{H}), 8.18(\mathrm{~s}, 1 \mathrm{H}), 7.93(\mathrm{~s}, 2 \mathrm{H}), 7.58(\mathrm{~s}, 1 \mathrm{H}), 5.61(\mathrm{~s}, 1 \mathrm{H})$, 5.20-5.06 (m, 1H), 4.70-4.56 (m, 1H), 4.33-4.19 (m, 1H), 3.66-3.50 (m, 1H), 2.44-2.28 (m, 1H), 2.16$2.07(\mathrm{~m}, 1 \mathrm{H}), 2.05-1.05$ (comp, 34H); ${ }^{13} \mathrm{C} \mathrm{NMR}\left(125 \mathrm{MHz}, \mathrm{CDCl}_{3}\right) \delta 180.38,178.22,140.41,131.99(\mathrm{q}$, $\left.J_{C-F}=33.4 \mathrm{~Hz}\right), 123.33,123.20\left(\mathrm{q}, J_{C-F}=272.9 \mathrm{~Hz}\right), 118.09,62.07,61.65,57.59,56.82,34.42,33.79$, $32.83,32.69,32.28,32.02,26.48,26.23,26.04,25.05,24.80,24.45 ; \mathrm{m} / z\left(\right.$ ESI-MS) $663.2[\mathrm{M}-\mathrm{H}]^{-}$. 
1-((1R,2R)-2-thioureidocyclohexyl)-3-(4-(trifluoromethyl)phenyl)thiourea: Following a procedure

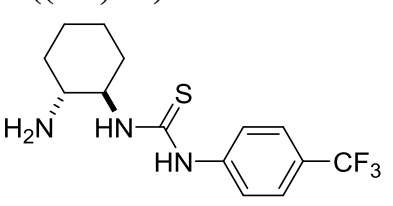

for a closely related product, ${ }^{16}(1 R, 2 R)$-cyclohexane-1,2-diamine $(2.24 \mathrm{~g}, 19.6$ mmol, 1.5 equiv), dissolved in a minimum amount of $\mathrm{CH}_{2} \mathrm{Cl}_{2}$ (ca. $1 \mathrm{~mL}$ ), was cooled to $0{ }^{\circ} \mathrm{C}$. 1-Isothiocyanato-4-(trifluoromethyl)benzene $(2.66 \mathrm{~g}, 13.1$ mmol, 1 equiv), dissolved in $262 \mathrm{~mL} \mathrm{CH}_{2} \mathrm{Cl}_{2}$, was added dropwise over 1

hour. The resulting mixture was allowed to warm to room temperature and concentrated under reduced pressure. Purification by flash chromatography on silica gel afforded the desired product as a white solid in $87 \%$ yield; $\mathrm{mp}=144-146^{\circ} \mathrm{C} ; \mathrm{R}_{\mathrm{f}}=0.10\left(\mathrm{MeOH} / \mathrm{CH}_{2} \mathrm{Cl}_{2} / \mathrm{Et}_{3} \mathrm{~N} \mathrm{10:89:1}\right.$ $\mathrm{v} / \mathrm{v} / \mathrm{v}) ;[\alpha]_{\mathrm{D}}{ }^{20}+125.5\left(\mathrm{c} 0.5, \mathrm{CHCl}_{3}\right) ; \mathrm{IR}(\mathrm{KBr}) 3259,2937,2854,1613,1523,1341,1161,1111,1068$, $1008,825,708 \mathrm{~cm}^{-1} ;{ }^{1} \mathrm{H}$ NMR $\left(500 \mathrm{MHz},\left(\mathrm{CD}_{3}\right)_{2} \mathrm{SO}\right) \delta 7.79(\operatorname{app~d}, \mathrm{J}=8.2 \mathrm{~Hz}, 2 \mathrm{H}), 7.60(\operatorname{app~d}, \mathrm{J}=8.4$ $\mathrm{Hz}, 2 \mathrm{H}), 4.08-3.75(\mathrm{~m}, 1 \mathrm{H}), 2.62-2.52(\mathrm{~m}, 1 \mathrm{H}), 2.23-1.99(\mathrm{~m}, 1 \mathrm{H}), 1.92-1.78(\mathrm{~m}, 1 \mathrm{H}), 1.73-1.50$ (comp, $2 \mathrm{H}), 1.34-0.98$ (comp, $4 \mathrm{H})$; ${ }^{13} \mathrm{C}$ NMR $\left(125 \mathrm{MHz},\left(\mathrm{CD}_{3}\right)_{2} \mathrm{SO}\right) \delta 180.31,143.76,125.36,124.46\left(\mathrm{q}, J_{C-F}=\right.$ $271.3 \mathrm{~Hz}), 123.25\left(\mathrm{q}, J_{C-F}=31.9 \mathrm{~Hz}\right), 121.57,60.08,54.02,35.05,31.05,24.62$; $\mathrm{m} / z$ (ESI-MS) $318.1[\mathrm{M}$ $+\mathrm{H}]^{-}$.

1,1-dicyclooctyl-3-((1R,2R)-2-(3-(4-(trifluoromethyl)phenyl)thioureido)cyclohexyl)thiourea (21):

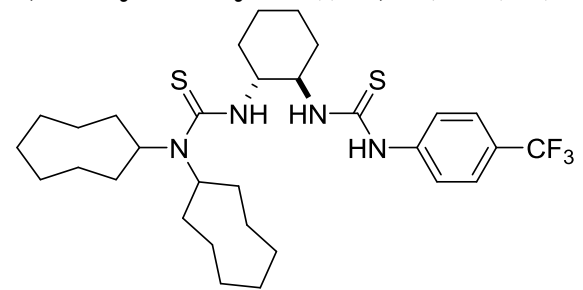
Following general procedure $\mathbf{B}$ (using 2.5 equivalents of dicyclooctylamine ${ }^{15}$ ), the reaction was stirred at $50^{\circ} \mathrm{C}$ for 24 hours to afford $\mathbf{2 l}$ as a white solid in $65 \%$ yield; $\mathrm{mp}=110-112^{\circ} \mathrm{C} ; \mathrm{R}_{\mathrm{f}}=$ $0.31\left(\right.$ Hexanes/EtOAc 85:15 v/v); $[\alpha]_{\mathrm{D}}{ }^{20}-25.1\left(\mathrm{c} 0.5, \mathrm{CHCl}_{3}\right) ;$ IR (KBr) 3432, 3222, 2924, 2847, 1614, 1522, 1441, 1324, 1124, $1067,1011,948,828 \mathrm{~cm}^{-1} ;{ }^{1} \mathrm{H}$ NMR $\left(500 \mathrm{MHz}, \mathrm{CDCl}_{3}\right) \delta 7.96(\mathrm{~s}$, $1 \mathrm{H}), 7.71(\mathrm{~s}, 1 \mathrm{H}), 7.59(\operatorname{app~d}, J=8.3 \mathrm{~Hz}, 2 \mathrm{H}), 7.44(\operatorname{app~d}, J=$ $8.3 \mathrm{~Hz}, 2 \mathrm{H}), 5.65(\mathrm{~s}, 1 \mathrm{H}), 5.18-5.05(\mathrm{~m}, 1 \mathrm{H}), 4.81-4.68(\mathrm{~m}, 1 \mathrm{H}), 4.35-4.22(\mathrm{~m}, 1 \mathrm{H}), 3.67-3.50(\mathrm{~m}, 1 \mathrm{H})$, 2.44-2.31 (m, 1H), 2.15-2.06 (m, 1H), 2.05-1.23 (m, 34H); ${ }^{13} \mathrm{C} \mathrm{NMR}\left(125 \mathrm{MHz}, \mathrm{CDCl}_{3}\right) \delta 179.85$, $178.51,140.74,127.17$ (q, $\left.J_{C-F}=33.3 \mathrm{~Hz}\right), 126.56,124.06$ (q, $\left.J_{C-F}=270 \mathrm{~Hz}\right), 123.36,61.71,61.48,57.33$, $56.60,34.33,33.82,32.94,32.66,32.36,32.07,26.59,26.27,25.05,24.90,24.52 ; \mathrm{m} / \mathrm{z}$ (ESI-MS) 595.3 $[\mathrm{M}-\mathrm{H}]^{-}$. 


\section{Preparation and characterization data of products:}

\section{General procedure $\mathrm{C}$ for the enantioselective oxa-Pictet-Spengler reaction}

To a flame dried vial was added amine salt $\mathbf{1 d} \cdot \mathbf{H C l}(5.3 \mathrm{mg}, 0.025 \mathrm{mmol}, 10 \mathrm{~mol} \%)$, thiourea catalyst $\mathbf{2 l}$ (15 mg, $0.025 \mathrm{mmol}, 10 \mathrm{~mol} \%)$, tryptophol (40 mg, $0.25 \mathrm{mmol}, 1$ equiv), $4 \AA \mathrm{MS}$ (100 mg) and dry toluene $(5 \mathrm{~mL}, 0.05 \mathrm{M})$. The resulting mixture was stirred under nitrogen and cooled to $-30{ }^{\circ} \mathrm{C}$ over 15 minutes. Aldehyde ( $0.3 \mathrm{mmol}, 1.2$ equiv) was then added and the reaction was stirred for the indicated time before being quenched with triethylamine $(40 \mu \mathrm{L})$. The resulting mixture was directly purified by flash chromatography on silica gel topped with Celite.

(R)-1-(4-chlorophenyl)-1,3,4,9-tetrahydropyrano[3,4-b]indole (3a): Following general procedure C,

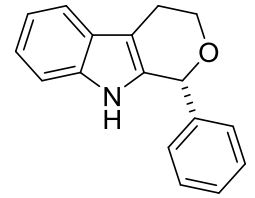
the reaction was run for 2 days and 3a was obtained as a white solid in $90 \%$ yield; $\mathrm{mp}$ $=153-155^{\circ} \mathrm{C} ; \mathrm{R}_{\mathrm{f}}=0.32($ Hexanes $/$ EtOAc $90: 10 \mathrm{v} / \mathrm{v}) ;[\alpha]^{\mathrm{D}}{ }_{20}-15.7\left(\mathrm{c} 0.5, \mathrm{CHCl}_{3}, 91 \%\right.$ ee); IR (KBr) 3397, 3032, 2909, 2837, 1446, 1311, 1273, 1251, 1136, 1083, 1048, 978, $738,693,470 \mathrm{~cm}^{-1} ;{ }^{1} \mathrm{H} \mathrm{NMR}\left(500 \mathrm{MHz}, \mathrm{CDCl}_{3}\right) \delta 7.57(\mathrm{app} \mathrm{dd}, J=7.0,2.1 \mathrm{~Hz}, 1 \mathrm{H})$, 7.46 (br s, 1H), 7.42-7.36 (comp, 5H), 7.25-7.19 (m, 1H), 7.19-7.11 (comp, 2H), 5.80 (app t, $J=1.9 \mathrm{~Hz}, 1 \mathrm{H}), 4.34(\mathrm{ddd}, J=11.2,5.4,2.9 \mathrm{~Hz}, 1 \mathrm{H}), 4.00(\mathrm{ddd}, J=11.3,9.6,4.0 \mathrm{~Hz}, 1 \mathrm{H}), 3.12$ (dddd, $J=15.2,9.7,5.4,2.1 \mathrm{~Hz}, 1 \mathrm{H}), 2.84(\mathrm{dddd}, J=15.4,4.1,3.0,1.9 \mathrm{~Hz}, 1 \mathrm{H}) ;{ }^{13} \mathrm{C}$ NMR $(125 \mathrm{MHz}$, $\left.\mathrm{CDCl}_{3}\right) \delta 139.59,136.16,133.74,129.06,128.94,128.57,127.14,122.08,119.78,118.47,111.13,108.91$, 76.28, 65.00, 22.44; $\mathrm{m} / z$ (ESI-MS) $250.2[\mathrm{M}+\mathrm{H}]^{+}$; HPLC: Daicel Chiralpak AD-H, $n$-hexane $/ i-\mathrm{PrOH}=$ $90 / 10$, Flow rate $=1 \mathrm{~mL} / \mathrm{min}, \mathrm{UV}=280.16 \mathrm{~nm}, \mathrm{t}_{\mathrm{R}}=14.7 \mathrm{~min}$ (minor) and $\mathrm{t}_{\mathrm{R}}=19.6 \mathrm{~min}$ (major).

The absolute configuration was assigned by analogy.

(R)-1-(4-fluorophenyl)-1,3,4,9-tetrahydropyrano[3,4-b]indole (3b): Following general procedure C, the reaction was run for 2 days and $\mathbf{3 b}$ was obtained as a white solid in $82 \%$ yield; $\mathrm{mp}$ $=116-118^{\circ} \mathrm{C} ; \mathrm{R}_{\mathrm{f}}=0.21\left(\right.$ Hexanes/EtOAc 90:10 v/v); $[\alpha]_{20}^{\mathrm{D}}-8.1\left(\mathrm{c} 0.5, \mathrm{CHCl}_{3}, 90 \%\right.$ ee); IR (KBr) 3389, 3292, 3047, 2957, 2914, 2847, 1713, 1606, 1509, 1446, 1224, $1153,1071,1043,830,743 \mathrm{~cm}^{-1} ;{ }^{1} \mathrm{H}$ NMR $\left(500 \mathrm{MHz}, \mathrm{CDCl}_{3}\right) \delta 7.59-7.55(\mathrm{~m}, 1 \mathrm{H})$, 7.44 (br s, 1H), 7.38-7.32 (comp, 2H), 7.27-7.23 (m, 1H), 7.21-7.12 (comp, 2H), 7.11$7.04($ comp, $2 \mathrm{H}), 5.78(\mathrm{t}, J=1.9 \mathrm{~Hz}, 1 \mathrm{H}), 4.31(\mathrm{ddd}, J=11.3,5.4,3.0 \mathrm{~Hz}, 1 \mathrm{H}), 3.99$ (ddd, $J=11.3,9.6,4.0 \mathrm{~Hz}, 1 \mathrm{H}), 3.10$ (dddd, $J=15.2,9.6,5.4,2.1 \mathrm{~Hz}, 1 \mathrm{H}), 2.84$ (dddd, $J=15.4,4.0,3.0$, $1.8 \mathrm{~Hz}, 1 \mathrm{H}) ;{ }^{13} \mathrm{C}$ NMR $\left(125 \mathrm{MHz}, \mathrm{CDCl}_{3}\right) \delta 163.03\left(\mathrm{~d}, J_{C-F}=247.7 \mathrm{~Hz}\right), 136.05,135.37\left(\mathrm{~d}, J_{C-F}=3.2 \mathrm{~Hz}\right)$, $133.28,130.28\left(\mathrm{~d}, J_{C-F}=8.3 \mathrm{~Hz}\right), 126.96,122.11,119.76,118.39,115.71\left(\mathrm{~d}, J_{C-F}=21.6 \mathrm{~Hz}\right), 111.01$, 109.01, 75.37, 64.82, 22.26; $\mathrm{m} / z$ (ESI-MS) $268.2[\mathrm{M}+\mathrm{H}]^{+}$; HPLC: Daicel Chiralpak AD-H, $n$-hexane $/ i$ $\mathrm{PrOH}=90 / 10$, Flow rate $=1 \mathrm{~mL} / \mathrm{min}, \mathrm{UV}=280.16 \mathrm{~nm}, \mathrm{t}_{\mathrm{R}}=15.7$ min (major) and $\mathrm{t}_{\mathrm{R}}=18.2 \mathrm{~min}$ (minor).

The absolute configuration was assigned by analogy.

(R)-1-(4-chlorophenyl)-1,3,4,9-tetrahydropyrano[3,4-b]indole (3c): Following general procedure C,

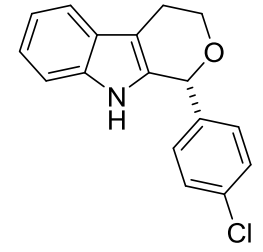
the reaction was run for 2 days and $3 \mathbf{c}$ was obtained as a white solid in $91 \%$ yield; $\mathrm{mp}$ $=101-103{ }^{\circ} \mathrm{C} ; \mathrm{R}_{\mathrm{f}}=0.28($ Hexanes $/$ EtOAc $90: 10 \mathrm{v} / \mathrm{v}) ;[\alpha]_{20}^{\mathrm{D}}-9.3\left(\mathrm{c} 0.5, \mathrm{CHCl}_{3}, 94 \%\right.$ ee); IR (KBr) 3393, 3307, 3052, 2919, 2834, 1713, 1611, 1490, 1451, 1246, 1087, $1015,815,743,510 \mathrm{~cm}^{-1} ;{ }^{1} \mathrm{H} \mathrm{NMR}\left(500 \mathrm{MHz}, \mathrm{CDCl}_{3}\right) \delta 7.57(\operatorname{app~dd}, J=7.6,1.2 \mathrm{~Hz}$, 1H), 7.45 (br s, 1H), 7.38-7.34 (comp, 2H), 7.33-7.29 (comp, 2H), 7.26-7.22 (m, 1H), 7.20-7.13 (comp, 2H), 5.76 (app t, $J=1.9 \mathrm{~Hz}, 1 \mathrm{H}), 4.30(\mathrm{ddd}, J=11.3,5.4,3.1 \mathrm{~Hz}$, $1 \mathrm{H}), 3.98$ (ddd, $J=11.4,9.5,4.1 \mathrm{~Hz}, 1 \mathrm{H}), 3.09$ (dddd, $J=15.0,9.5,5.3,2.0 \mathrm{~Hz}, 1 \mathrm{H}), 2.90-2.79(\mathrm{~m}, 1 \mathrm{H})$; ${ }^{13} \mathrm{C}$ NMR $\left(125 \mathrm{MHz}, \mathrm{CDCl}_{3}\right) \delta 138.14,136.20,134.91,133.14,129.93,129.11,127.06,122.29,119.92$, 118.54, 111.16, 109.13, 75.47, 64.90, 22.37; $\mathrm{m} / z$ (ESI-MS) $284.2[\mathrm{M}+\mathrm{H}]^{+}$; HPLC: Daicel Chiralpak 
AS-H, $n$-hexane $/ i-\mathrm{PrOH}=93 / 7$, Flow rate $=0.2 \mathrm{~mL} / \mathrm{min}, \mathrm{UV}=280.16 \mathrm{~nm}, \mathrm{t}_{\mathrm{R}}=66.4 \mathrm{~min}($ minor $)$ and $\mathrm{t}_{\mathrm{R}}=$ 69.8 min (major).

The absolute configuration was assigned by analogy.

(R)-1-(4-bromophenyl)-1,3,4,9-tetrahydropyrano[3,4-b]indole (3d): Following general procedure C,

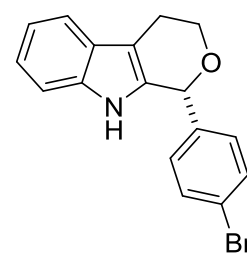
the reaction was run for 2 days and $\mathbf{3 d}$ was obtained as a white solid in $91 \%$ yield; $\mathrm{mp}$ $=116-118^{\circ} \mathrm{C} ; \mathrm{R}_{\mathrm{f}}=0.25\left(\right.$ Hexanes/EtOAc 90:10 v/v); $[\alpha]^{\mathrm{D}}{ }_{20}-7.2\left(\mathrm{c} 0.5, \mathrm{CHCl}_{3}, 93 \%\right.$ ee); IR (KBr) 3394, 3312, 3062, 2962, 2914, 2852, 2369, 2341, 1716, 1506, 1481, $1451,1253,1073,1043,1011,810,735,508 \mathrm{~cm}^{-1}$; ${ }^{1} \mathrm{H}$ NMR $\left(500 \mathrm{MHz}, \mathrm{CDCl}_{3}\right) \delta$ 7.59-7.55 (m, 1H), 7.53-7.49 (comp, 2H), 7.44 (br s, 1H), 7.26-7.23 (comp, 3H), $\mathrm{Br} \quad 7.20-7.12(\mathrm{comp}, 2 \mathrm{H}), 5.75(\operatorname{app} \mathrm{t}, J=1.9 \mathrm{~Hz}, 1 \mathrm{H}), 4.30(\mathrm{ddd}, J=11.3,5.4,3.0 \mathrm{~Hz}$, $1 \mathrm{H}), 3.98$ (ddd, $J=11.3,9.5,4.1 \mathrm{~Hz}, 1 \mathrm{H}), 3.09$ (dddd, $J=15.4,9.5,5.4,2.0 \mathrm{~Hz}, 1 \mathrm{H}$ ), 2.84 (dddd, $J=$ 15.5, 4.1, 3.1, $1.9 \mathrm{~Hz}, 1 \mathrm{H}) ;{ }^{13} \mathrm{C}$ NMR $\left(125 \mathrm{MHz}, \mathrm{CDCl}_{3}\right) \delta 138.65,136.20,133.07,132.08,130.23$, $127.05,123.11,122.31,119.92,118.55,111.16,109.13,75.53,64.91,22.37 ; \mathrm{m} / \mathrm{z}$ (ESI-MS) $328.3\left({ }^{79} \mathrm{Br}\right)$ $[\mathrm{M}+\mathrm{H}]^{+}, 330.3\left({ }^{81} \mathrm{Br}\right)[\mathrm{M}+\mathrm{H}]^{+}$; HPLC: Daicel Chiralpak AS-H, $n$-hexane/i-PrOH $=90 / 10$, Flow rate $=$ $0.2 \mathrm{~mL} / \mathrm{min}, \mathrm{UV}=280.16 \mathrm{~nm}, \mathrm{t}_{\mathrm{R}}=55.0 \mathrm{~min}$ (minor) and $\mathrm{t}_{\mathrm{R}}=59.5 \mathrm{~min}$ (major).

The absolute configuration of $\mathbf{3 d}$ was assigned by X-ray crystallography:

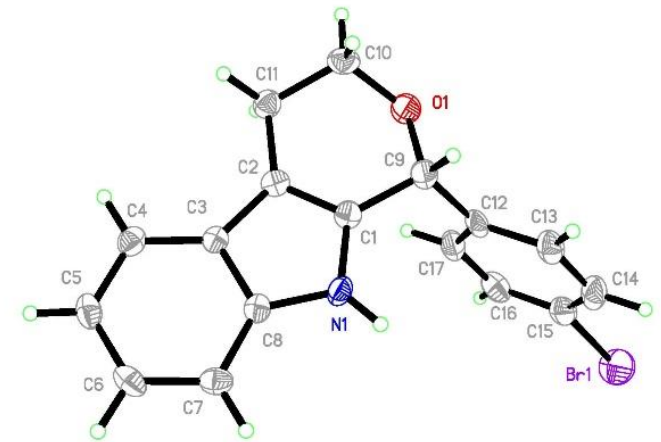

Compound 3d was crystallized from $\mathrm{MeOH}$ through slow evaporation at room temperature. The requisite CIF has been submitted to the journal.

(R)-1-(p-tolyl)-1,3,4,9-tetrahydropyrano[3,4-b]indole (3e): Following general procedure $\mathbf{C}$, the reaction was run for 2 days and $\mathbf{3 e}$ was obtained as a white solid in $90 \%$ yield; $\mathrm{mp}=$ $179-180^{\circ} \mathrm{C} ; \mathrm{R}_{\mathrm{f}}=0.24$ (Hexanes/EtOAc 90:10 v/v); $[\alpha]_{20}^{\mathrm{D}}-2.4\left(\mathrm{c} 0.5, \mathrm{CHCl}_{3}, 95 \%\right.$ ee); IR (KBr) 3394, 2947, 2909, 2859, 2819, 1446, 1298, 1081, 1041, 808, 740, 455 $\mathrm{cm}^{-1} ;{ }^{1} \mathrm{H}$ NMR $\left(500 \mathrm{MHz}, \mathrm{CDCl}_{3}\right) \delta 7.59(\operatorname{app~d}, J=6.5 \mathrm{~Hz}, 1 \mathrm{H}), 7.49$ (br s, $\left.1 \mathrm{H}\right)$, 7.33-7.24 (comp, 2H), 7.24-7.19 (comp, 3H), 7.19-7.13 (comp, 2H), 5.76 (s, 1H), 4.32 (ddd, $J=11.4,5.5,3.2 \mathrm{~Hz}, 1 \mathrm{H}), 3.99$ (ddd, $J=11.3,9.6,4.1 \mathrm{~Hz}, 1 \mathrm{H}), 3.16-3.06$ $(\mathrm{m}, 1 \mathrm{H}), 2.89-2.81(\mathrm{~m}, 1 \mathrm{H}), 2.40(\mathrm{~s}, 3 \mathrm{H}) ;{ }^{13} \mathrm{C} \mathrm{NMR}\left(125 \mathrm{MHz}, \mathrm{CDCl}_{3}\right) \delta 138.86,136.61,136.11,133.90$, 129.53, 128.55, 127.13, 121.96, 119.69, 118.40, 111.10, 108.82, 75.98, 64.78, 22.42, 21.35; $\mathrm{m} / \mathrm{z}(\mathrm{ESI}-\mathrm{MS})$ $264.1[\mathrm{M}+\mathrm{H}]^{+}$; HPLC: Daicel Chiralpak AD-H, $n$-hexane $/ i-\mathrm{PrOH}=90 / 10$, Flow rate $=1 \mathrm{~mL} / \mathrm{min}$, UV $=280.16 \mathrm{~nm}, \mathrm{t}_{\mathrm{R}}=11.9 \min \left(\right.$ minor) and $\mathrm{t}_{\mathrm{R}}=18.3 \min$ (major).

The absolute configuration was assigned by analogy. 
(R)-1-(4-methoxyphenyl)-1,3,4,9-tetrahydropyrano[3,4-b]indole (3f): Following general procedure C,

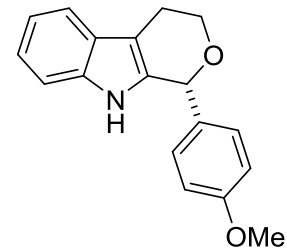
the reaction was run for 3 days and $\mathbf{3 f}$ was obtained as a white solid in $84 \%$ yield; $\mathrm{mp}=115-117^{\circ} \mathrm{C} ; \mathrm{R}_{\mathrm{f}}=0.26\left(\right.$ Hexanes/EtOAc 80:20 v/v); $[\alpha]_{20}^{\mathrm{D}}+0.6\left(\mathrm{c} 0.5, \mathrm{CHCl}_{3}\right.$, $84 \%$ ee); IR (KBr) 3392, 2949, 2907, 2837, 1611, 1448, 1301, 1243, 1173, 1078, $1033,968,830,735,465,508,473 \mathrm{~cm}^{-1} ;{ }^{1} \mathrm{H}$ NMR (500 MHz, $\left.\mathrm{CDCl}_{3}\right) \delta 7.59-7.54$ (m, 1H), 7.46 (br s, 1H), 7.32-7.27 (comp, 2H), 7.25-7.22 (m, 1H), 7.19-7.11 (comp, 2H), 6.92-6.88 (comp, 2H), 5.76 (app t, $J=1.9 \mathrm{~Hz}, 1 \mathrm{H}), 4.31$ (ddd, $J=11.2,5.3,3.1$

$\mathrm{Hz}, 1 \mathrm{H}$ ), 3.98 (ddd, $J=11.2,9.4,4.1 \mathrm{~Hz}, 1 \mathrm{H}), 3.82$ (s, 3H), 3.09 (dddd, $J=15.0,9.5,5.4,2.1 \mathrm{~Hz}, 1 \mathrm{H}$ ), 2.842 (dddd, $J=15.4,4.0,3.1,1.8 \mathrm{~Hz}, 1 \mathrm{H}) ;{ }^{13} \mathrm{C} \mathrm{NMR}\left(125 \mathrm{MHz}, \mathrm{CDCl}_{3}\right) \delta 160.17,136.11,133.98$, $131.75,129.99,127.14,121.99,119.71,118.41,114.22,111.10,108.94,75.73,64.79,55.45,22.44 ; \mathrm{m} / \mathrm{z}$ (ESI-MS) $280.1[\mathrm{M}+\mathrm{H}]^{+}$; HPLC: Daicel Chiralpak AD-H, $n$-hexane $/ i-\mathrm{PrOH}=90 / 10$, Flow rate $=1$ $\mathrm{mL} / \mathrm{min}, \mathrm{UV}=280.16 \mathrm{~nm}, \mathrm{t}_{\mathrm{R}}=20.8 \min$ (minor) and $\mathrm{t}_{\mathrm{R}}=31.3 \min$ (major).

The absolute configuration was assigned by analogy.

(R)-1-(3-chlorophenyl)-1,3,4,9-tetrahydropyrano[3,4-b]indole (3g): Following general procedure C, the reaction was run for 2 days and $\mathbf{3 g}$ was obtained as a white solid in $73 \%$ yield; $\mathrm{mp}$ $=157-160{ }^{\circ} \mathrm{C} ; \mathrm{R}_{\mathrm{f}}=0.18($ Hexanes$/$ EtOAc $90: 10 \mathrm{v} / \mathrm{v}) ;[\alpha]^{\mathrm{D}}{ }_{20}-3.6\left(\mathrm{c} 0.5, \mathrm{CHCl}_{3}, 82 \%\right.$ ee); IR (KBr) 3394, 3052, 2957, 2922, 2837, 1718, 1621, 1596, 1571, 1461, 1433, $1296,1253,1078,1041,855,790,740,705,690 \mathrm{~cm}^{-1} ;{ }^{1} \mathrm{H}$ NMR $\left(500 \mathrm{MHz}, \mathrm{CDCl}_{3}\right) \delta$ 7.57 (ddd, $J=7.4,1.5,0.8 \mathrm{~Hz}, 1 \mathrm{H}), 7.45$ (br s, 1H), 7.39-7.34 (comp, 2H), 7.34-7.30 (m, 1H), 7.29-7.23 (comp, 2H), 7.21-7.11 (comp, 2H), 5.77 (app t, $J=2.0 \mathrm{~Hz}, 1 \mathrm{H})$, 4.32 (ddd, $J=11.3,5.4,3.0 \mathrm{~Hz}, 1 \mathrm{H}), 3.99$ (ddd, $J=11.3,9.5,4.1 \mathrm{~Hz}, 1 \mathrm{H}), 3.10$ (dddd, $J=15.1,9.5,5.4$, $2.0 \mathrm{~Hz}, 1 \mathrm{H}), 2.89-2.78(\mathrm{~m}, 1 \mathrm{H}) ;{ }^{13} \mathrm{C}$ NMR $\left(125 \mathrm{MHz}, \mathrm{CDCl}_{3}\right) \delta 141.75,136.29,134.95,132.94,130.22$, $129.23,128.59,127.10,126.62,122.36,119.96,118.58,111.21,109.19,75.61,65.00,22.38$; $\mathrm{m} / \mathrm{z}$ (ESIMS) $284.1[\mathrm{M}+\mathrm{H}]^{+}$; HPLC: Daicel Chiralpak AS-H, $n$-hexane $/ i-\mathrm{PrOH}=90 / 10$, Flow rate $=1 \mathrm{~mL} / \mathrm{min}$, $\mathrm{UV}=280.16 \mathrm{~nm}, \mathrm{t}_{\mathrm{R}}=8.3 \mathrm{~min}$ (minor) and $\mathrm{t}_{\mathrm{R}}=10.6 \mathrm{~min}$ (major).

The absolute configuration was assigned by analogy.

(R)-1-(3-iodophenyl)-1,3,4,9-tetrahydropyrano[3,4-b]indole (3h): Following general procedure $\mathbf{C}$, the

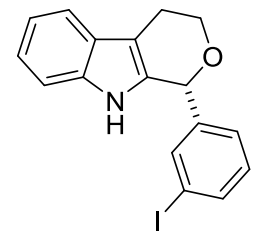
reaction was run for 3 days and $\mathbf{3 h}$ was obtained as a white solid in $78 \%$ yield; $\mathrm{mp}=$ $158-160{ }^{\circ} \mathrm{C} ; \mathrm{R}_{\mathrm{f}}=0.17($ Hexanes/EtOAc $90: 10 \mathrm{v} / \mathrm{v}) ;[\alpha]_{20}^{\mathrm{D}}+3.1\left(\mathrm{c} 0.5, \mathrm{CHCl}_{3}, 85 \%\right.$ ee); IR (KBr) 3399, 2957, 2914, 2864, 2822, 1568, 1468, 1296, 1076, 1056, 800, 745, 675, $465 \mathrm{~cm}^{-1} ;{ }^{1} \mathrm{H}$ NMR $\left(500 \mathrm{MHz}, \mathrm{CDCl}_{3}\right) \delta 7.78-7.67$ (comp, 2H), 7.58 (app d, $J=7.2$ $\mathrm{Hz}, 1 \mathrm{H}), 7.48($ br s, $1 \mathrm{H}), 7.34(\operatorname{app~d}, J=7.8 \mathrm{~Hz}, 1 \mathrm{H}), 7.25(\operatorname{app~d}, J=8.2 \mathrm{~Hz}, 1 \mathrm{H})$, 7.22-7.14 (comp, 2H), 7.12 (app t, $J=7.7 \mathrm{~Hz}, 1 \mathrm{H}), 5.71(\operatorname{app~t}, J=1.9 \mathrm{~Hz}, 1 \mathrm{H}), 4.31$ (ddd, $J=11.3,5.3,2.9 \mathrm{~Hz}, 1 \mathrm{H}), 3.98(\mathrm{ddd}, J=11.3,9.6,4.1 \mathrm{~Hz}, 1 \mathrm{H}), 3.11$ (dddd, $J=15.2,9.7,5.4,2.1$ $\mathrm{Hz}, 1 \mathrm{H}), 2.89-2.79(\mathrm{~m}, 1 \mathrm{H}) ;{ }^{13} \mathrm{C} \mathrm{NMR}\left(125 \mathrm{MHz}, \mathrm{CDCl}_{3}\right) \delta 141.94,138.11,137.32,136.22,132.87$, $130.62,127.77,127.01,122.30,119.90,118.54,111.21,109.10,94.86,75.46,64.99,22.33 ; \mathrm{m} / \mathrm{z}(\mathrm{ESI}-\mathrm{MS})$ $376.0[\mathrm{M}+\mathrm{H}]^{+}$; HPLC: Daicel Chiralpak AD-H, $n$-hexane $/ i-\mathrm{PrOH}=90 / 10$, Flow rate $=1 \mathrm{~mL} / \mathrm{min}$, UV $=280.16 \mathrm{~nm}, \mathrm{t}_{\mathrm{R}}=14.2 \min$ (minor) and $\mathrm{t}_{\mathrm{R}}=17.1 \mathrm{~min}$ (major).

The absolute configuration was assigned by analogy. 
(R)-1-(m-tolyl)-1,3,4,9-tetrahydropyrano[3,4-b]indole (3i): Following general procedure C, the

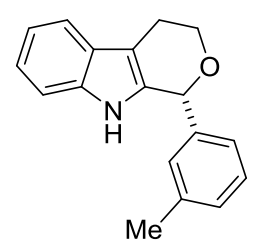
reaction was run for 2 days and $\mathbf{3 i}$ was obtained as a white solid in $86 \%$ yield; $\mathrm{mp}=$ $126-128^{\circ} \mathrm{C} ; \mathrm{R}_{\mathrm{f}}=0.17$ (Hexanes/EtOAc 90:10 v/v); $[\alpha]_{20}^{\mathrm{D}}-4.9\left(\mathrm{c} 0.5, \mathrm{CHCl}_{3}, 86 \%\right.$ ee); IR (KBr) 3399, 3024, 2962, 2917, 2832, 1611, 1466, 1443, 1368, 1291, 1276, 1138, $1076,1043,748,690 \mathrm{~cm}^{-1}$; ${ }^{1} \mathrm{H}$ NMR $\left(500 \mathrm{MHz}, \mathrm{CDCl}_{3}\right) \delta 7.61-7.55(\mathrm{~m}, 1 \mathrm{H}), 7.47(\mathrm{br}$ s, 1H), 7.32-7.27 (m, 1H), 7.27-7.22 (m, 1H), 7.22-7.12 (comp, 5H), 5.76 (app t, $J=$ $2.0 \mathrm{~Hz}, 1 \mathrm{H}), 4.35(\mathrm{ddd}, J=11.5,5.5,2.8 \mathrm{~Hz}, 1 \mathrm{H}), 4.05-3.93(\mathrm{~m}, 1 \mathrm{H}), 3.19-3.05(\mathrm{~m}$, $1 \mathrm{H}), 2.84$ (dddd, $J=15.5,3.9,2.7,1.4 \mathrm{~Hz}, 1 \mathrm{H}), 2.35(\mathrm{~s}, 3 \mathrm{H}) ;{ }^{13} \mathrm{C}$ NMR $\left(125 \mathrm{MHz}, \mathrm{CDCl}_{3}\right) \delta 139.48$, 138.76, 136.14, 133.91, 129.84, 129.10, 128.78, 127.17, 125.67, 122.02, 119.75, 118.45, 111.14, 108.79, 76.37, 65.10, 22.44, 21.51; m/z (ESI-MS) $264.2[\mathrm{M}+\mathrm{H}]^{+}$; HPLC: Daicel Chiralpak AD-H, $n$-hexane/i$\mathrm{PrOH}=90 / 10$, Flow rate $=1 \mathrm{~mL} / \mathrm{min}, \mathrm{UV}=254.16 \mathrm{~nm}, \mathrm{t}_{\mathrm{R}}=11.4 \mathrm{~min}$ (minor) and $\mathrm{t}_{\mathrm{R}}=19.7$ min (major).

The absolute configuration was assigned by analogy.

(R)-1-(3-methoxyphenyl)-1,3,4,9-tetrahydropyrano[3,4-b]indole (3j): Following general procedure $\mathbf{C}$,

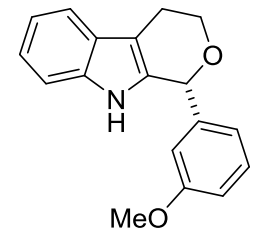
the reaction was run for 2 days and $\mathbf{3 j}$ was obtained as a white solid in $82 \%$ yield; $\mathrm{mp}=$ $107-109^{\circ} \mathrm{C} ; \mathrm{R}_{\mathrm{f}}=0.14($ Hexanes/EtOAc $90: 10 \mathrm{v} / \mathrm{v}) ;[\alpha]_{20}^{\mathrm{D}}-2.6\left(\mathrm{c} 0.5, \mathrm{CHCl}_{3}, 86 \%\right.$ ee $)$; IR (KBr) 3284, 3049, 2939, 2894, 2844, 1606, 1581, 1491, 1448, 1276, 1228, 1151, $1041,875,805,763,690 \mathrm{~cm}^{-1} ;{ }^{1} \mathrm{H}$ NMR $\left(500 \mathrm{MHz}, \mathrm{CDCl}_{3}\right) \delta 7.56($ app dd, $J=7.5$, $1.5 \mathrm{~Hz}, 1 \mathrm{H}), 7.47$ (br s, 1H), 7.34-7.27 (m, 1H), 7.24 (app dd, $J=6.9,1.4 \mathrm{~Hz}, 1 \mathrm{H})$, 7.19-7.11 (comp, 2H), 6.98 (app dt, $J=7.5,1.2 \mathrm{~Hz}, 1 \mathrm{H}), 6.95-6.89$ (comp, 2H), 5.77 (app t, $J=1.9 \mathrm{~Hz}, 1 \mathrm{H}), 4.36(\mathrm{ddd}, J=11.3,5.5,2.8 \mathrm{~Hz}, 1 \mathrm{H}), 4.00(\mathrm{ddd}, J=11.3,9.8,4.0 \mathrm{~Hz}, 1 \mathrm{H}), 3.78$ (s, 3H), 3.12 (dddd, $J=15.3,9.8,5.5,2.1 \mathrm{~Hz}, 1 \mathrm{H}), 2.83$ (dddd, $J=15.4,4.3,2.7,1.8 \mathrm{~Hz}, 1 \mathrm{H}$ ); ${ }^{13} \mathrm{C}$ NMR $\left(125 \mathrm{MHz}, \mathrm{CDCl}_{3}\right) \delta 160.19,141.19,136.20,133.72,129.95,127.22,122.09,120.70,119.78,118.46$, 114.78, 113.67, 111.16, 108.76, 76.27, 65.12, 55.44, 22.43; $\mathrm{m} / \mathrm{z}$ (ESI-MS) $280.3\left[\mathrm{M}+\mathrm{H}^{+}{ }^{+}\right.$; HPLC: Daicel Chiralpak AD-H, $n$-hexane $/ i-\mathrm{PrOH}=90 / 10$, Flow rate $=1 \mathrm{~mL} / \mathrm{min}, \mathrm{UV}=280.16 \mathrm{~nm}, \mathrm{t}_{\mathrm{R}}=15.8$ $\min \left(\right.$ minor) and $t_{R}=30.7 \min$ (major).

The absolute configuration was assigned by analogy.

(R)-1-(3-(benzyloxy)phenyl)-1,3,4,9-tetrahydropyrano[3,4-b]indole (3k): Following general

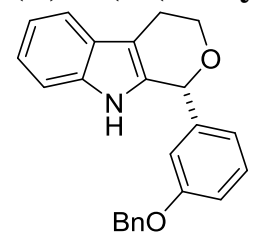
procedure $\mathbf{C}$, the reaction was run for 3 days and $\mathbf{3 k}$ was obtained as a white solid in $87 \%$ yield; $m p=154-156{ }^{\circ} \mathrm{C} ; \quad \mathrm{R}_{\mathrm{f}}=0.15\left(\right.$ Hexanes $/$ EtOAc 90:10 v/v); $[\alpha]_{20}^{\mathrm{D}}-9.0(\mathrm{c} 0.5$, $\mathrm{CHCl}_{3}, 90 \%$ ee); IR (KBr) 3439, 3059, 3032, 2959, 2917, 2857, 1586, 1491, 1446, $1256,1153,1071,1038,735,693 \mathrm{~cm}^{-1} ;{ }^{1} \mathrm{H} \mathrm{NMR}\left(500 \mathrm{MHz}, \mathrm{CDCl}_{3}\right) \delta 7.57-7.54(\mathrm{~m}$, 1H), 7.44-7.37 (comp, 3H), 7.37-7.33 (comp, 2H), 7.33-7.28 (comp, 2H), 7.25-7.22 $(\mathrm{m}, 1 \mathrm{H}), 7.19-7.11$ (comp, 2H), 7.03-6.94 (comp, 3H), 5.77 (app t, $J=1.8 \mathrm{~Hz}, 1 \mathrm{H})$, $5.05(\mathrm{~d}, J=11.6 \mathrm{~Hz}, 1 \mathrm{H}), 5.02(\mathrm{~d}, J=11.5 \mathrm{~Hz}, 1 \mathrm{H}), 4.34(\mathrm{ddd}, J=11.4,5.4,2.8 \mathrm{~Hz}, 1 \mathrm{H}), 4.02-3.95(\mathrm{~m}$, $1 \mathrm{H}), 3.15-3.04(\mathrm{~m}, 1 \mathrm{H}), 2.87-2.76(\mathrm{~m}, 1 \mathrm{H}) ;{ }^{13} \mathrm{C} \mathrm{NMR}\left(125 \mathrm{MHz}, \mathrm{CDCl}_{3}\right) \delta 159.31,141.19,136.90$, 136.17, 133.65, 130.04, 128.70, 128.14, 127.70, 127.19, 122.10, 120.95, 119.80, 118.48, 115.61, 114.61, 111.19, 108.78, 76.21, 70.15, 65.13, 22.43; $\mathrm{m} / \mathrm{z}$ (ESI-MS) $356.3[\mathrm{M}+\mathrm{H}]^{+}$; HPLC: Daicel Chiralpak AS-H, $n$-hexane $/ i$-PrOH $=90 / 10$, Flow rate $=1 \mathrm{~mL} / \mathrm{min}, \mathrm{UV}=254.16 \mathrm{~nm}, \mathrm{t}_{\mathrm{R}}=12.0 \mathrm{~min}($ minor $)$ and $\mathrm{t}_{\mathrm{R}}=$ 15.8 min (major).

The absolute configuration was assigned by analogy. 
(R)-1-(naphthalen-2-yl)-1,3,4,9-tetrahydropyrano[3,4-b]indole (3l): Following general procedure C,

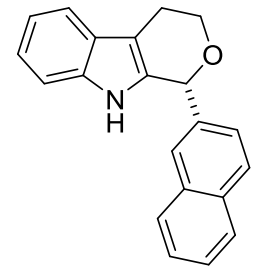
the reaction was run for 2 days and $\mathbf{3 l}$ was obtained as a white solid in $86 \%$ yield; $\mathrm{mp}=$ $185-187^{\circ} \mathrm{C} ; \mathrm{R}_{\mathrm{f}}=0.21($ Hexanes/EtOAc $90: 10 \mathrm{v} / \mathrm{v}) ;[\alpha]_{20}^{\mathrm{D}}-104.6\left(\mathrm{c} 0.5, \mathrm{CHCl}_{3}, 88 \%\right.$ ee); IR (KBr) 3378, 3042, 2961, 2889, 2839, 1506, 1466, 1449, 1370, 1293, 1253, $1141,1073,1048,863,824,739,484 \mathrm{~cm}^{-1} ;{ }^{1} \mathrm{H}$ NMR $\left(500 \mathrm{MHz}, \mathrm{CDCl}_{3}\right) \delta 7.90-7.81$ (comp, 4H), 7.64-7.58 (m, 1H), 7.56-7.50 (comp, 2H), 7.49-7.44 (comp, 2H), 7.237.14 (comp, 3H), 5.94 (app t, $J=1.9 \mathrm{~Hz}, 1 \mathrm{H}$ ), 4.37 (ddd, $J=11.3,5.4,3.0 \mathrm{~Hz}, 1 \mathrm{H}$ ), 4.04 (ddd, $J=11.2,9.5,4.1 \mathrm{~Hz}, 1 \mathrm{H}), 3.16$ (dddd, $J=15.2,9.5,5.4,2.1 \mathrm{~Hz}, 1 \mathrm{H}), 2.95-$ $2.79(\mathrm{~m}, 1 \mathrm{H}) ;{ }^{13} \mathrm{C}$ NMR $\left(125 \mathrm{MHz}, \mathrm{CDCl}_{3}\right) \delta 137.02,136.21,133.73,133.28,128.94,128.22,127.89$, 127.72, 127.18, 126.61, 126.50, 125.99, 122.11, 119.80, 118.50, 111.18, 108.94, 76.36, 64.95, 22.47; m/z (ESI-MS) $300.1[\mathrm{M}+\mathrm{H}]^{+}$; HPLC: Daicel Chiralpak AD-H, $n$-hexane $/ i-\mathrm{PrOH}=90 / 10$, Flow rate $=1$ $\mathrm{mL} / \mathrm{min}, \mathrm{UV}=280.16 \mathrm{~nm}, \mathrm{t}_{\mathrm{R}}=20.0 \min$ (minor) and $\mathrm{t}_{\mathrm{R}}=25.0$ min (major).

The absolute configuration was assigned by analogy.

(R)-1-(o-tolyl)-1,3,4,9-tetrahydropyrano[3,4-b]indole (3m): Following general procedure $\mathrm{C}$, the

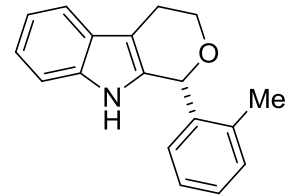
reaction was run for 2 days and $\mathbf{3 m}$ was obtained as a white solid in $91 \%$ yield; $\mathrm{mp}=$ 98-100 ${ }^{\circ} \mathrm{C} ; \mathrm{R}_{\mathrm{f}}=0.26($ Hexanes/EtOAc $90: 10 \mathrm{v} / \mathrm{v}) ;[\alpha]_{20}^{\mathrm{D}}-4.5\left(\mathrm{c} 0.5, \mathrm{CHCl}_{3}, 48 \%\right.$ ee); IR (KBr) 3397, 3289, 3052, 2954, 2909, 2847, 1453, 1303, 1256, 1076, 1041, $740 \mathrm{~cm}^{-1} ;{ }^{1} \mathrm{H}$ NMR $\left(500 \mathrm{MHz}, \mathrm{CDCl}_{3}\right) \delta 7.60-7.54(\mathrm{~m}, 1 \mathrm{H}), 7.47$ (br s, $\left.1 \mathrm{H}\right), 7.30$ 7.22 (comp, 3H), 7.20-7.10 (comp, 4H), 6.02 (app t, $J=2.1 \mathrm{~Hz}, 1 \mathrm{H}$ ), 4.29 (ddd, $J=$ $11.3,5.2,3.5 \mathrm{~Hz}, 1 \mathrm{H}), 3.99$ (ddd, $J=11.4,9.0,4.1 \mathrm{~Hz}, 1 \mathrm{H}), 3.08$ (dddd, $J=16.1,9.0,5.3,1.9 \mathrm{~Hz}, 1 \mathrm{H}$ ), $2.87(\mathrm{app} d \mathrm{dd}, J=15.4,4.0,1.8 \mathrm{~Hz}, 1 \mathrm{H}), 2.42(\mathrm{~s}, 3 \mathrm{H}) ;{ }^{13} \mathrm{C}$ NMR $\left(125 \mathrm{MHz}, \mathrm{CDCl}_{3}\right) \delta 137.83,137.15$, 136.09, 133.58, 131.26, 129.49, 128.95, 127.15, 126.17, 121.99, 119.77, 118.39, 111.11, 109.21, 73.98, 64.70, 22.47, 19.14; $\mathrm{m} / \mathrm{z}$ (ESI-MS) $264.2[\mathrm{M}+\mathrm{H}]^{+}$; HPLC: Daicel Chiralpak AD-H, $n$-hexane $/ i$-PrOH $=90 / 10$, Flow rate $=1 \mathrm{~mL} / \mathrm{min}, \mathrm{UV}=280.16 \mathrm{~nm}, \mathrm{t}_{\mathrm{R}}=10.5 \mathrm{~min}$ (minor) and $\mathrm{t}_{\mathrm{R}}=16.6 \mathrm{~min}$ (major).

The absolute configuration was assigned by analogy.

(R)-7-chloro-1-phenyl-1,3,4,9-tetrahydropyrano[3,4-b]indole (3n): Following general procedure $\mathbf{C}$,<smiles>Clc1ccc2c3c([nH]c2c1)C(c1ccccc1)OCC3</smiles>
the reaction was run for 4 days and $\mathbf{3 n}$ was obtained as a white solid in $89 \%$ yield; $\mathrm{mp}=106-108^{\circ} \mathrm{C} ; \mathrm{R}_{\mathrm{f}}=0.18($ Hexanes $/$ EtOAc $90: 10 \mathrm{v} / \mathrm{v}) ;[\alpha]_{20}^{\mathrm{D}}+25.2\left(\mathrm{c} 0.5, \mathrm{CHCl}_{3}\right.$, $94 \%$ ee); IR (KBr) 3414, 3279, 2964, 2917, 2837, 1451, 1301, 1241, 1146, 1051, 903, 850, 803, 755, $690 \mathrm{~cm}^{-1}$; ${ }^{1} \mathrm{H}$ NMR $(500 \mathrm{MHz}) \delta 7.45(\mathrm{~s}, 1 \mathrm{H}), 7.44(\mathrm{br} \mathrm{s}, 1 \mathrm{H})$, 7.41-7.33 (comp, 5H), 7.22-7.19 (m, 1H), 7.11-7.08 (m, 1H), $5.76($ app t, $J=1.9 \mathrm{~Hz}, 1 \mathrm{H}), 4.32(\mathrm{ddd}, J=$ $11.4,5.5,2.9 \mathrm{~Hz}, 1 \mathrm{H}), 3.98(\mathrm{ddd}, J=11.3,9.8,4.0 \mathrm{~Hz}, 1 \mathrm{H}), 3.15-3.00(\mathrm{~m}, 1 \mathrm{H}), 2.85-2.72(\mathrm{~m}, 1 \mathrm{H}) ;{ }^{13} \mathrm{C}$ NMR (125 MHz, $\left.\mathrm{CDCl}_{3}\right)$ 139.27, 136.48, 134.47, 129.20, 129.03, 128.51, 127.82, 125.79, 120.46, 119.28, 111.14, 109.02, 76.14, 64.89, 22.30; m/z (ESI-MS) $284.2[\mathrm{M}+\mathrm{H}]^{+}$; HPLC: Daicel Chiralpak AD-H, $n$ hexane $/ i-\mathrm{PrOH}=90 / 10$, Flow rate $=1 \mathrm{~mL} / \mathrm{min}, \mathrm{UV}=254.16 \mathrm{~nm}, \mathrm{t}_{\mathrm{R}}=9.9 \mathrm{~min}\left(\right.$ minor) and $\mathrm{t}_{\mathrm{R}}=14.1 \mathrm{~min}$ (major).

The absolute configuration was assigned by analogy.

(R)-6-bromo-1-phenyl-1,3,4,9-tetrahydropyrano[3,4-b]indole (3o): Following general procedure C, $\mathrm{Br}$ the reaction was run for 4 days and $\mathbf{3 o}$ was obtained as a white solid in $91 \%$ yield; $\mathrm{mp}$ $=100-102{ }^{\circ} \mathrm{C} ; \mathrm{R}_{\mathrm{f}}=0.21($ Hexanes $/$ EtOAc $90: 10 \mathrm{v} / \mathrm{v}) ;[\alpha]_{20}^{\mathrm{D}}-62.5\left(\mathrm{c} 0.5, \mathrm{CHCl}_{3}, 92 \%\right.$ ee); IR (KBr) 3414, 3282, 2954, 2917, 2842, 1451, 1296, 1078, 1043, 986, 793 ,698 $\mathrm{cm}^{-1}$; ${ }^{1} \mathrm{H}$ NMR $(500 \mathrm{MHz}) \delta 7.67(\mathrm{~s}, 1 \mathrm{H}), 7.46$ (br s, 1H), 7.43-7.33 (comp, 5H), 7.25-7.21 (m, 1H), 7.12-7.08 (m, 1H), 5.78 (app t, $J=2.0 \mathrm{~Hz}, 1 \mathrm{H}), 4.33$ (ddd, $J=11.5,5.5,2.8 \mathrm{~Hz}, 1 \mathrm{H})$, 4.02-3.94 (m, 1H), 3.06 (dddd, $J=15.5,9.6,5.6,2.0 \mathrm{~Hz}, 1 \mathrm{H}), 2.81-2.74(\mathrm{~m}, 1 \mathrm{H}) ;{ }^{13} \mathrm{C} \mathrm{NMR}(125 \mathrm{MHz}$, $\left.\mathrm{CDCl}_{3}\right) \delta 139.25,135.24,134.78,129.21,129.06,128.98,128.47,124.88,121.22,113.04,112.52,108.66$, 
76.20, 65.00, 22.29; $\mathrm{m} / z$ (ESI-MS) $328.1\left({ }^{79} \mathrm{Br}\right)[\mathrm{M}+\mathrm{H}]^{+}, 330.1\left({ }^{81} \mathrm{Br}\right)[\mathrm{M}+\mathrm{H}]^{+}$; HPLC: Daicel Chiralpak AD-H, $n$-hexane $/ i$-PrOH $=90 / 10$, Flow rate $=1 \mathrm{~mL} / \mathrm{min}, \mathrm{UV}=280.16 \mathrm{~nm}, \mathrm{t}_{\mathrm{R}}=13.9 \mathrm{~min}$ (minor) and $t_{R}=21.1 \mathrm{~min}$ (major).

The absolute configuration was assigned by analogy.

(R)-5-methyl-1-phenyl-1,3,4,9-tetrahydropyrano[3,4-b]indole (3p): Following general procedure C,

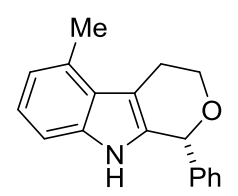
the reaction was run for 4 days and $\mathbf{3 p}$ was obtained as a white solid in $75 \%$ yield; $\mathrm{mp}=$ $155-158^{\circ} \mathrm{C} ; \mathrm{R}_{\mathrm{f}}=0.20($ Hexanes/EtOAc $90: 10 \mathrm{v} / \mathrm{v}) ;[\alpha]_{20}^{\mathrm{D}}-45.9\left(\mathrm{c} 0.5, \mathrm{CHCl}_{3}, 81 \%\right.$ ee); IR (KBr) 3294, 2979, 2937, 2849, 1443, 1373, 1333, 1141, 1073, 1041, 976, 750, 698 $\mathrm{cm}^{-1}$; ${ }^{1} \mathrm{H}$ NMR (500 MHz) $\delta$ 7.47-7.32 (comp, 6H), 7.08-6.98 (comp, 2H), 6.87-6.82 (m, $1 \mathrm{H}), 5.80(\operatorname{app~t}, J=1.9 \mathrm{~Hz}, 1 \mathrm{H}), 4.31(\mathrm{ddd}, J=11.4,5.5,2.9 \mathrm{~Hz}, 1 \mathrm{H}), 3.98(\mathrm{ddd}, J=$ 11.3, 9.7, 4.0 Hz, 1H), 3.35 (dddd, $J=15.3,9.8,5.5,2.2 \mathrm{~Hz}, 1 \mathrm{H}), 3.14-3.06(\mathrm{~m}, 1 \mathrm{H}), 2.69(\mathrm{~s}, 3 \mathrm{H}) ;{ }^{13} \mathrm{C}$ NMR $\left(125 \mathrm{MHz}, \mathrm{CDCl}_{3}\right) \delta 139.69,136.10,133.29,130.74,129.06,128.96,128.58,126.21,122.09$, 120.93, 109.15, 108.86, 76.44, 65.24, 24.95, 19.71; $\mathrm{m} / \mathrm{z}$ (ESI-MS) $264.1[\mathrm{M}+\mathrm{H}]^{+}$; HPLC: Daicel Chiralpak AD-H, $n$-hexane $/ i$-PrOH $=90 / 10$, Flow rate $=1 \mathrm{~mL} / \mathrm{min}, \mathrm{UV}=280.16 \mathrm{~nm}, \mathrm{t}_{\mathrm{R}}=19.1 \mathrm{~min}$ (major) and $t_{R}=26.7$ min (minor).

The absolute configuration was assigned by analogy.

9-methyl-1-phenyl-1,3,4,9-tetrahydropyrano[3,4-b]indole (3q): Following general procedure $\mathbf{C}$, the reaction was run at room temperature for 24 hours and $\mathbf{3 q}$ was obtained as a white solid in $62 \%$ yield; $m p=103-105^{\circ} \mathrm{C} ; \mathrm{R}_{\mathrm{f}}=0.36($ Hexanes/EtOAc 90:10 v/v); IR $(\mathrm{KBr}) 3029$, 2977, 2917, 2854, 1466, 1378, 1258, 1183, 1056, 898, 735, 698, 615, 503 $\mathrm{cm}^{-1}$; ${ }^{1} \mathrm{H}$ NMR (500 MHz) $\delta 7.63-7.60(\mathrm{~m}, 1 \mathrm{H}), 7.41-7.35$ (comp, 3H), 7.34-7.29 (comp, 2H), 7.29$7.28(\mathrm{~m}, 1 \mathrm{H}), 7.28-7.23(\mathrm{~m}, 1 \mathrm{H}), 7.20-7.15(\mathrm{~m}, 1 \mathrm{H}), 5.92($ app t, $J=1.3 \mathrm{~Hz}, 1 \mathrm{H}), 4.04$ (app dt, $J=11.4$, $5.6 \mathrm{~Hz}, 1 \mathrm{H}), 3.94(\mathrm{app} \mathrm{dt}, J=11.3,5.2 \mathrm{~Hz}, 1 \mathrm{H}), 3.28(\mathrm{~s}, 3 \mathrm{H}), 2.99-2.95$ (comp, 2H); ${ }^{13} \mathrm{C}$ NMR $(125$ $\left.\mathrm{MHz}, \mathrm{CDCl}_{3}\right) \delta 139.65,137.30,134.09,129.09,128.85,128.78,126.60,121.61,119.26,118.43,109.00$, 108.59, 74.66, 62.13, 30.29, 22.50; m/z (ESI-MS) $264.2[\mathrm{M}+\mathrm{H}]^{+}$; HPLC: Daicel Chiralpak AD-H, $n$ hexane $/ i-\mathrm{PrOH}=90 / 10$, Flow rate $=1 \mathrm{~mL} / \mathrm{min}, \mathrm{UV}=280.16 \mathrm{~nm}, \mathrm{t}_{\mathrm{R}}=9.8 \mathrm{~min}$ and $13.3 \mathrm{~min}$.

3-(2-(methoxy(phenyl)methoxy)ethyl)-1H-indole (4): To a solution of tryptophol $(0.5 \mathrm{~g}, 3.1 \mathrm{mmol}, 1$ OMe equiv) in $\mathrm{CH}_{2} \mathrm{Cl}_{2}(62 \mathrm{~mL}, 0.05 \mathrm{M})$ was added benzaldehyde dimethyl acetal $(0.70$

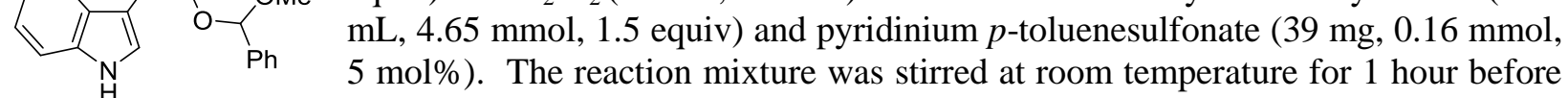
being quenched with trimethylamine $(0.1 \mathrm{~mL})$. The resulting mixture was then concentrated under reduced pressure and purified by flash chromatography on silica gel to afford $\mathbf{4}$ as a colorless oil in $16 \%$ yield. $R_{\mathrm{f}}=0.31$ (Hexanes/EtOAc 85:15 v/v); IR (film) 3409, 3054, 2927, 2872, 2361, 1453, 1418, 1353, 1203, 1098, 1043, 740, $703 \mathrm{~cm}^{-1}$; ${ }^{1} \mathrm{H}$ NMR (500 MHz) $\delta 7.97$ (br s, 1H), 7.637.60 (m, 1H), 7.51-7.46 (comp, 2H), 7.41-7.30 (comp, 4H), 7.20 (ddd, $J=8.1,7.0,1.2 \mathrm{~Hz}, 1 \mathrm{H}), 7.13$ $(\mathrm{ddd}, J=8.0,7.0,1.0 \mathrm{~Hz}, 1 \mathrm{H}), 7.05-7.03(\mathrm{~m}, 1 \mathrm{H}), 5.52(\mathrm{~s}, 1 \mathrm{H}), 3.90($ app dt, $J=9.4,7.2 \mathrm{~Hz}, 1 \mathrm{H}), 3.81$ (app dt, $J=9.5,7.2 \mathrm{~Hz}, 1 \mathrm{H}), 3.32(\mathrm{~s}, 3 \mathrm{H}), 3.15-3.10(\mathrm{comp}, 2 \mathrm{H}) ;{ }^{13} \mathrm{C}$ NMR $\left(125 \mathrm{MHz}, \mathrm{CDCl}_{3}\right) \delta 138.55$, 136.24, 128.51, 128.30, 127.65, 126.82, 122.14, 122.00, 119.33, 118.92, 113.01, 111.17, 102.69, 66.08, $52.80,25.93 ; \mathrm{m} / \mathrm{z}(\mathrm{ESI}-\mathrm{MS}) 250.1[\mathrm{M}-\mathrm{OMe}]^{+}$. 


\section{HPLC profiles of products}

HPLC profile of $\mathbf{3 a}$
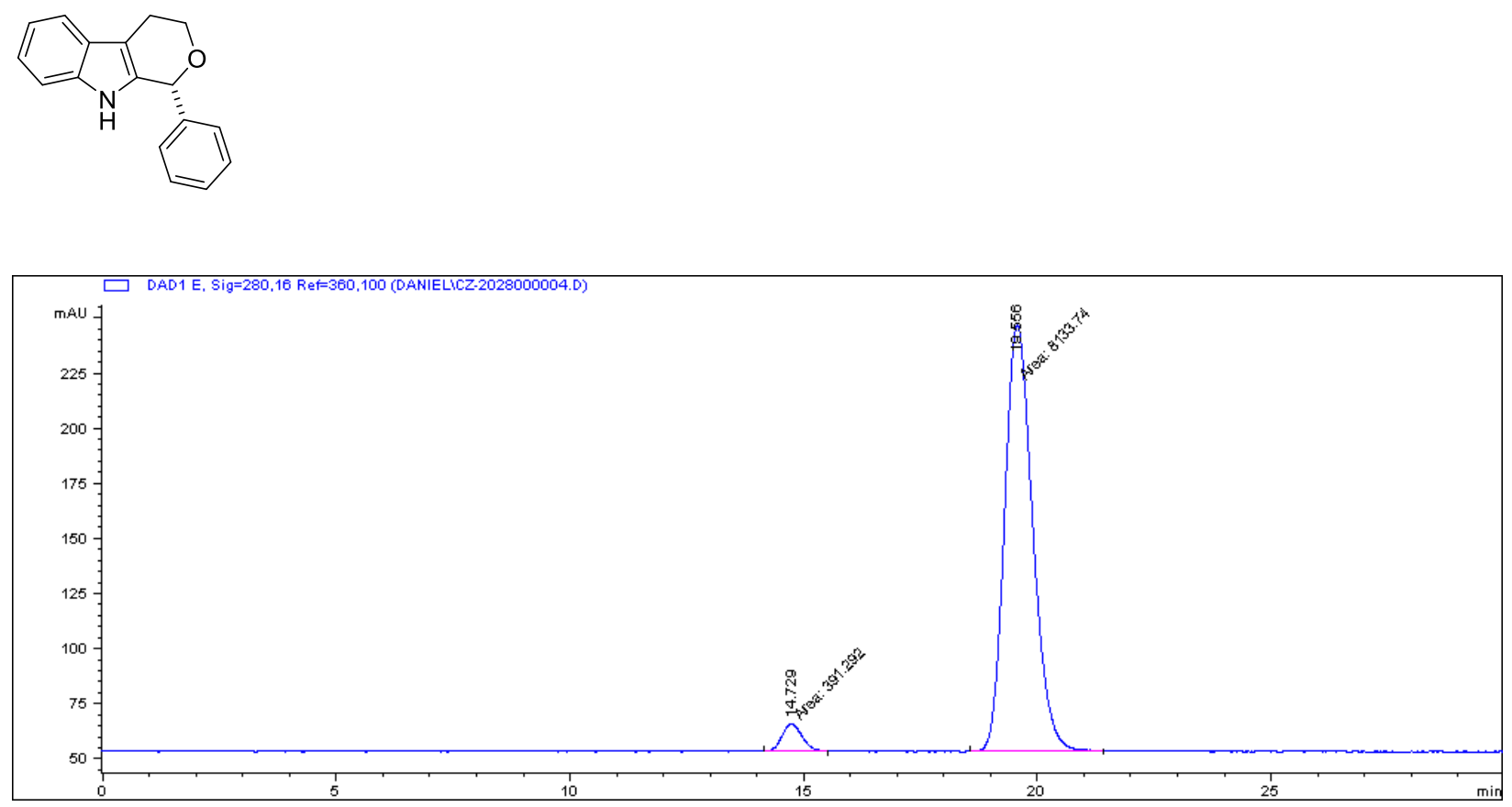

Racemic

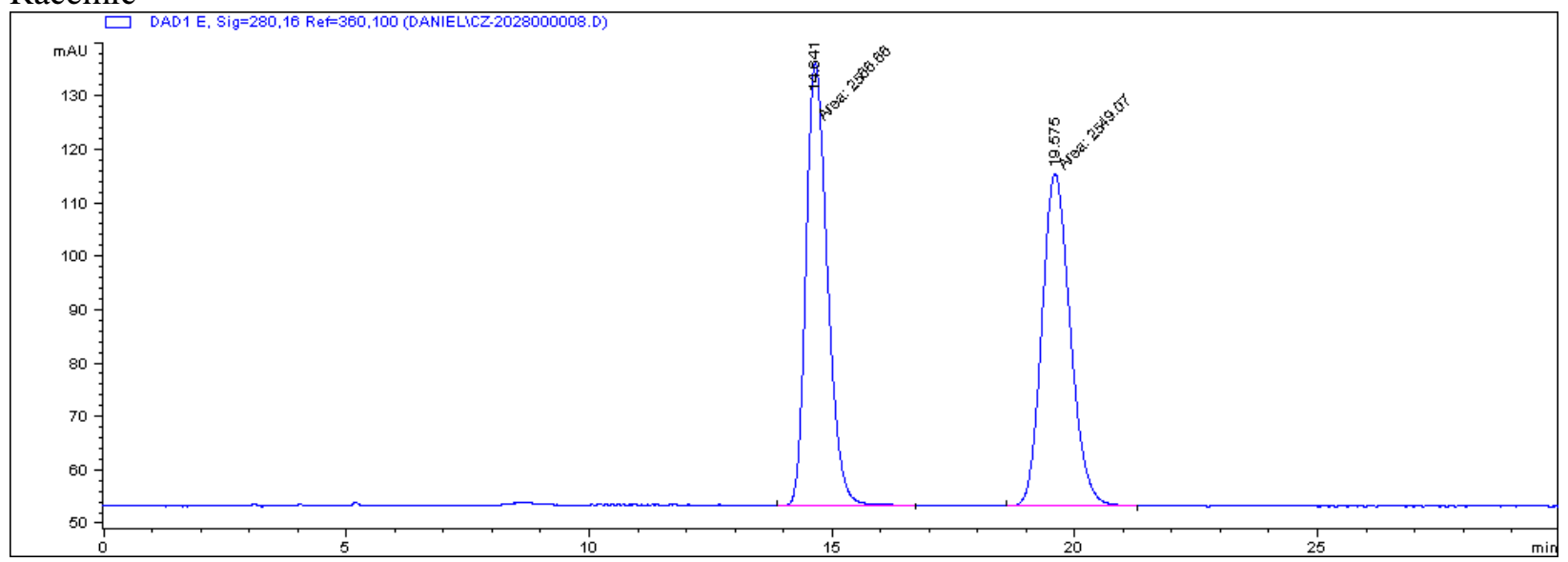


HPLC Profile of $\mathbf{3 b}$<smiles>Fc1ccc([C@H]2OCCc3c2[nH]c2ccccc32)cc1</smiles>

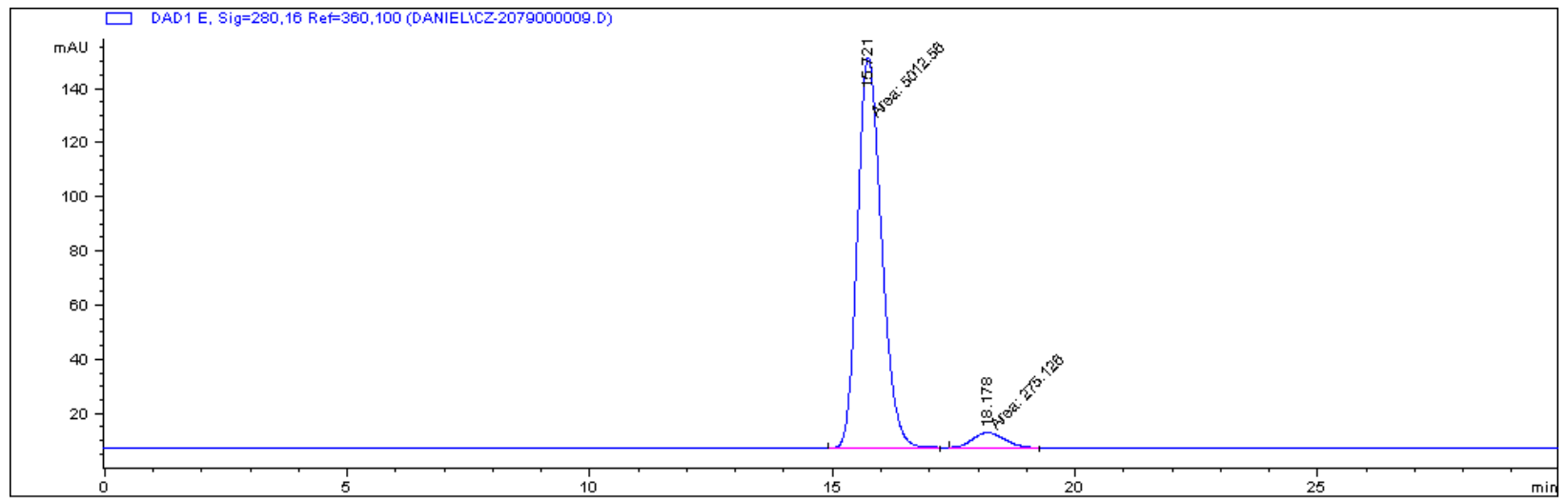

Racemic

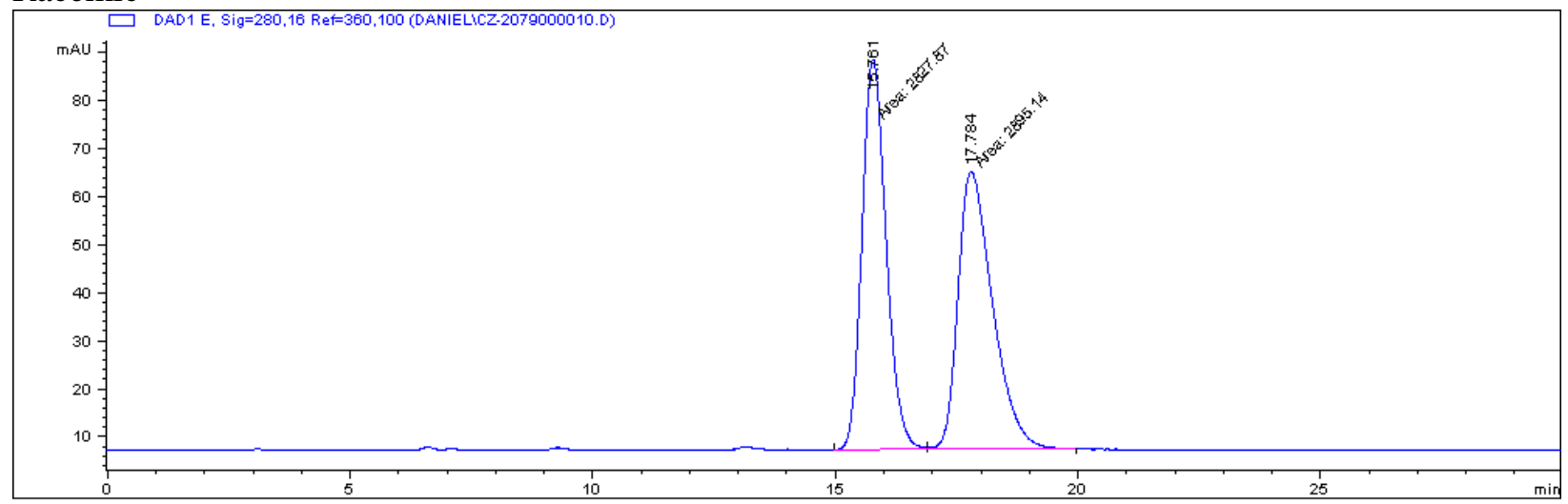


HPLC Profile of $\mathbf{3 c}$<smiles>Clc1ccc([C@@H]2OCCc3c2[nH]c2ccccc32)cc1</smiles>

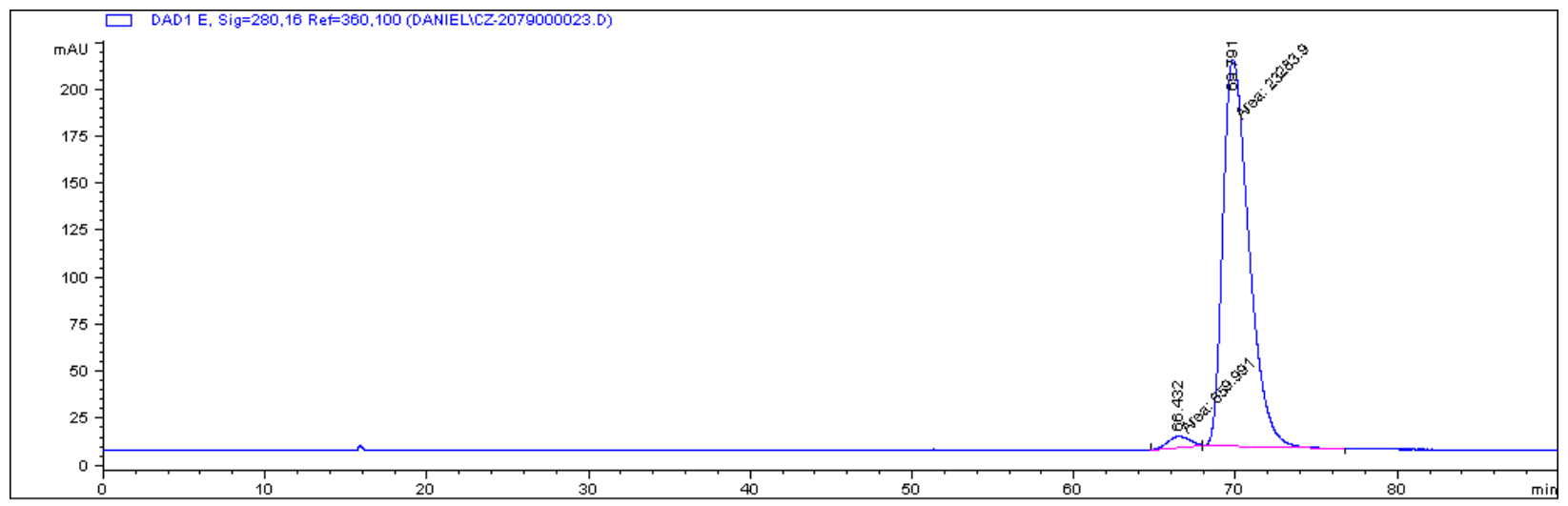

Racemic

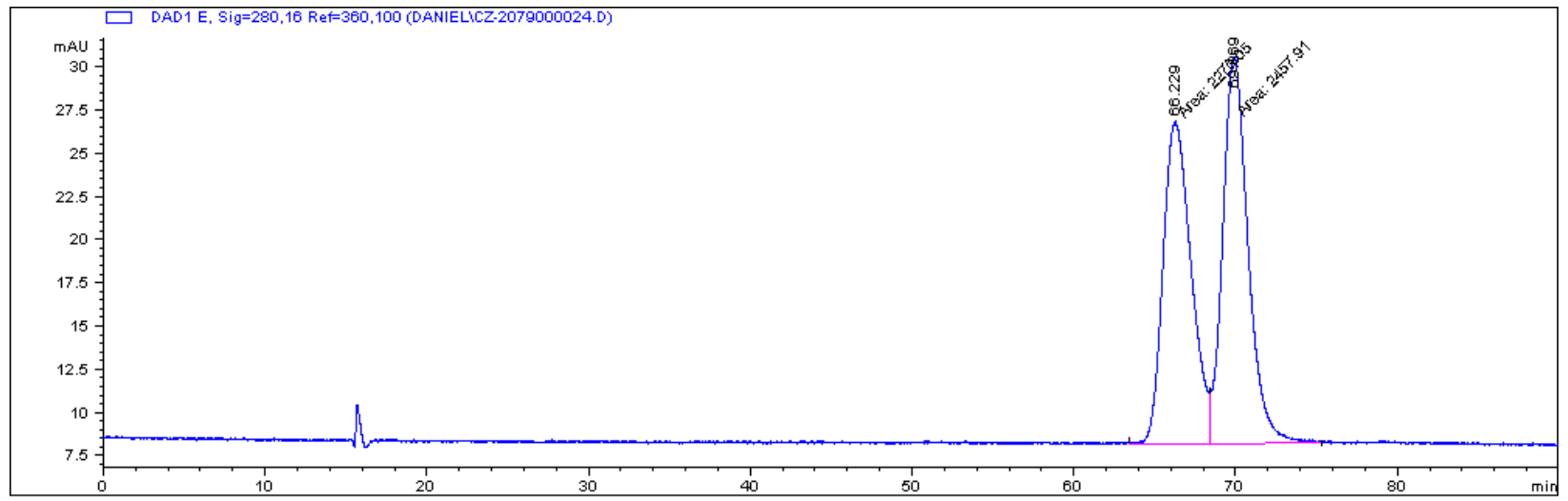


HPLC Profile of $\mathbf{3 d}$<smiles>Brc1ccc([C@@H]2OCCc3c2[nH]c2ccccc32)cc1</smiles>

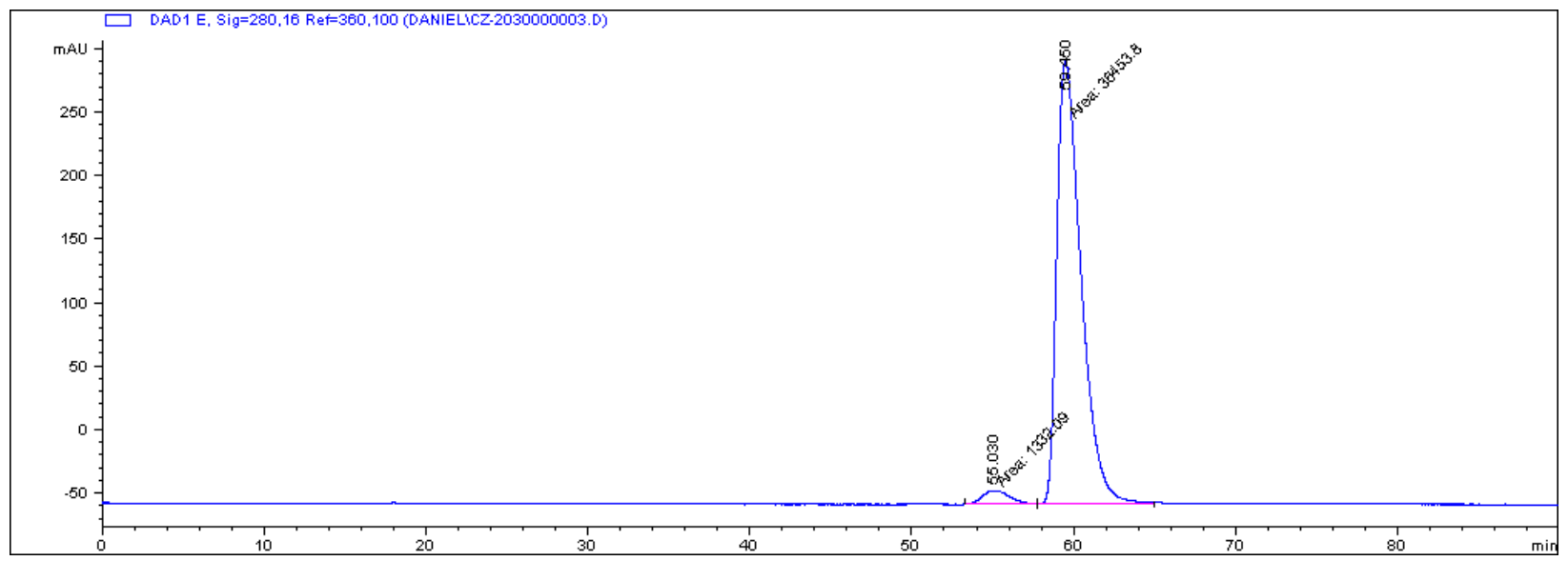

Racemic

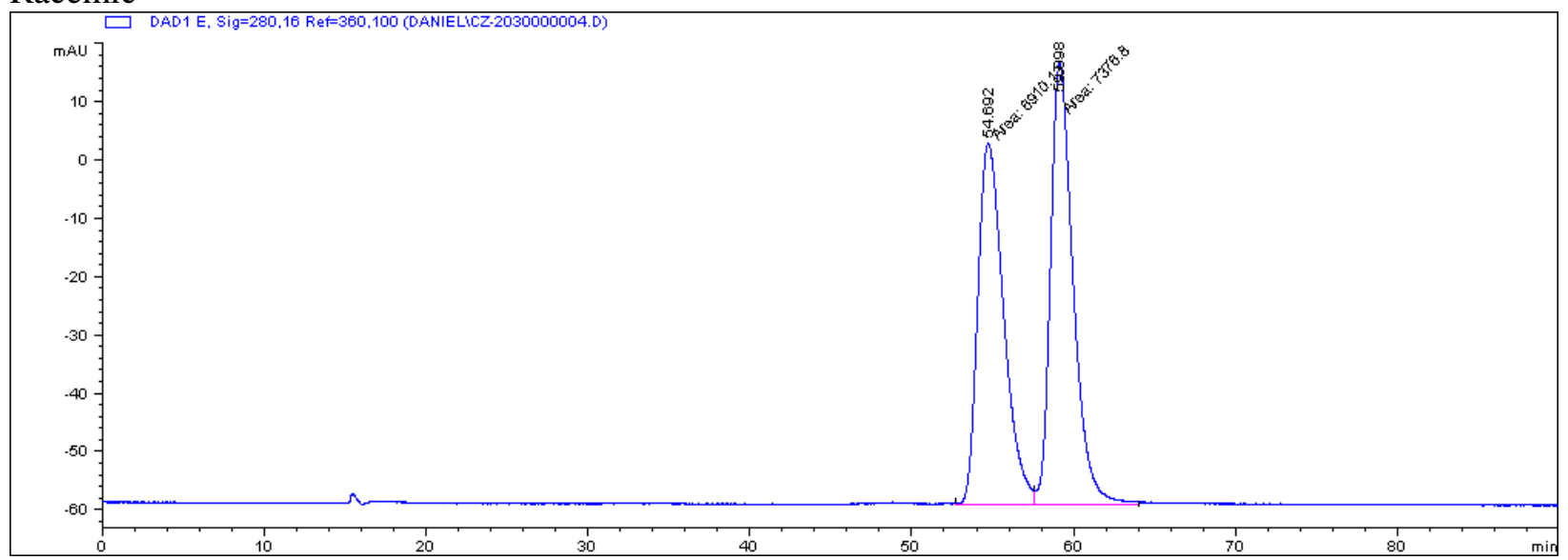


HPLC Profile of $\mathbf{3 e}$
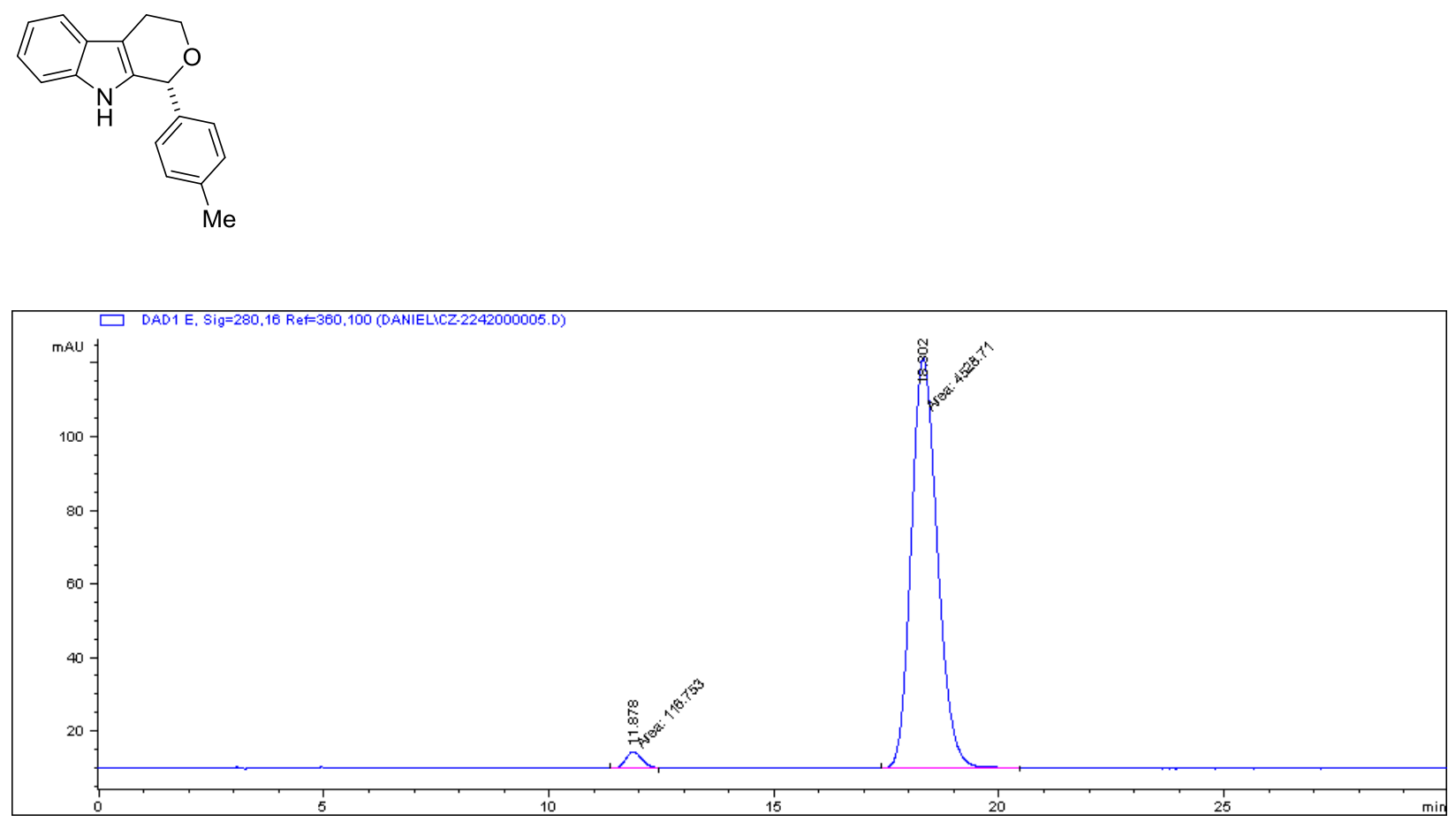

Racemic

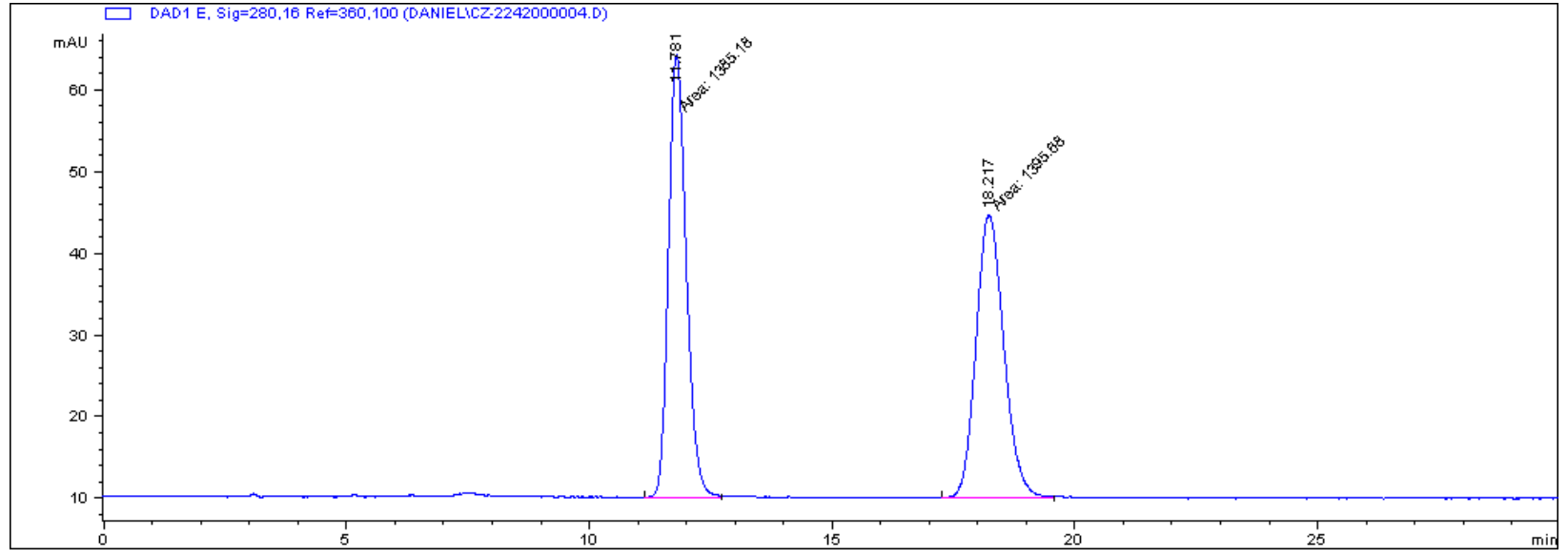


HPLC Profile of $\mathbf{3 f}$<smiles>COc1ccc([C@H]2OCCc3c2[nH]c2ccccc32)cc1</smiles>

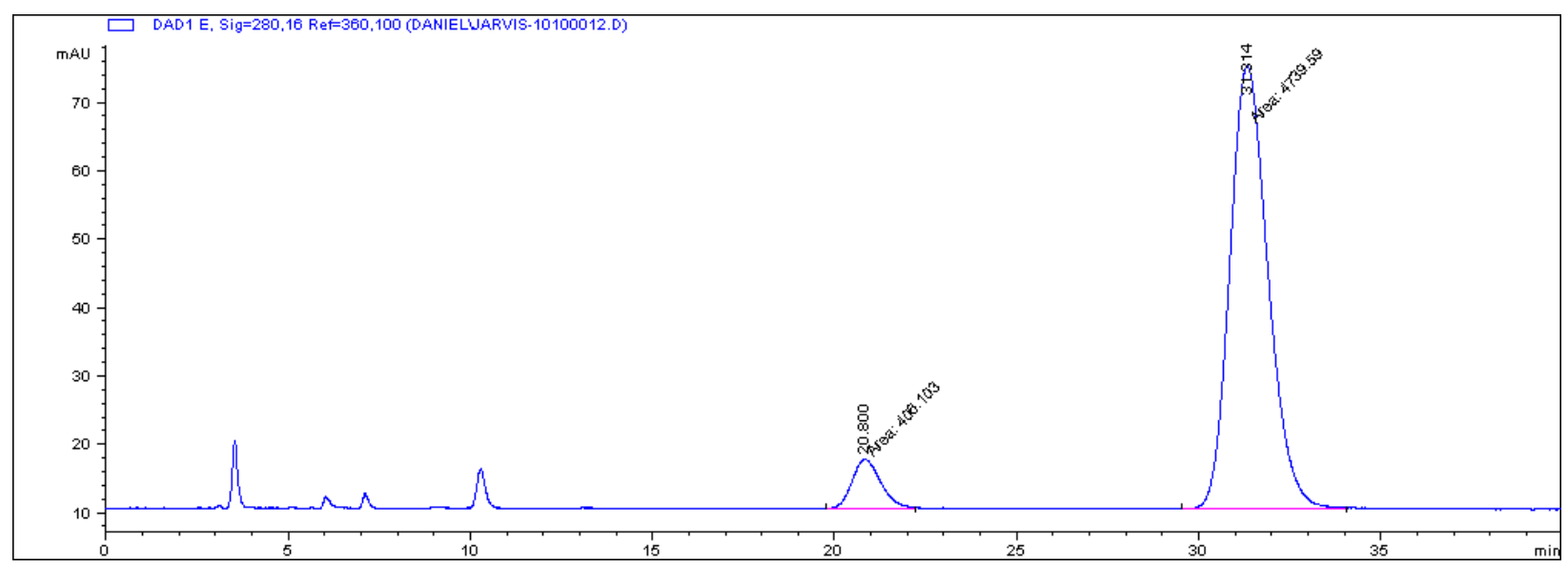

\section{Racemic}

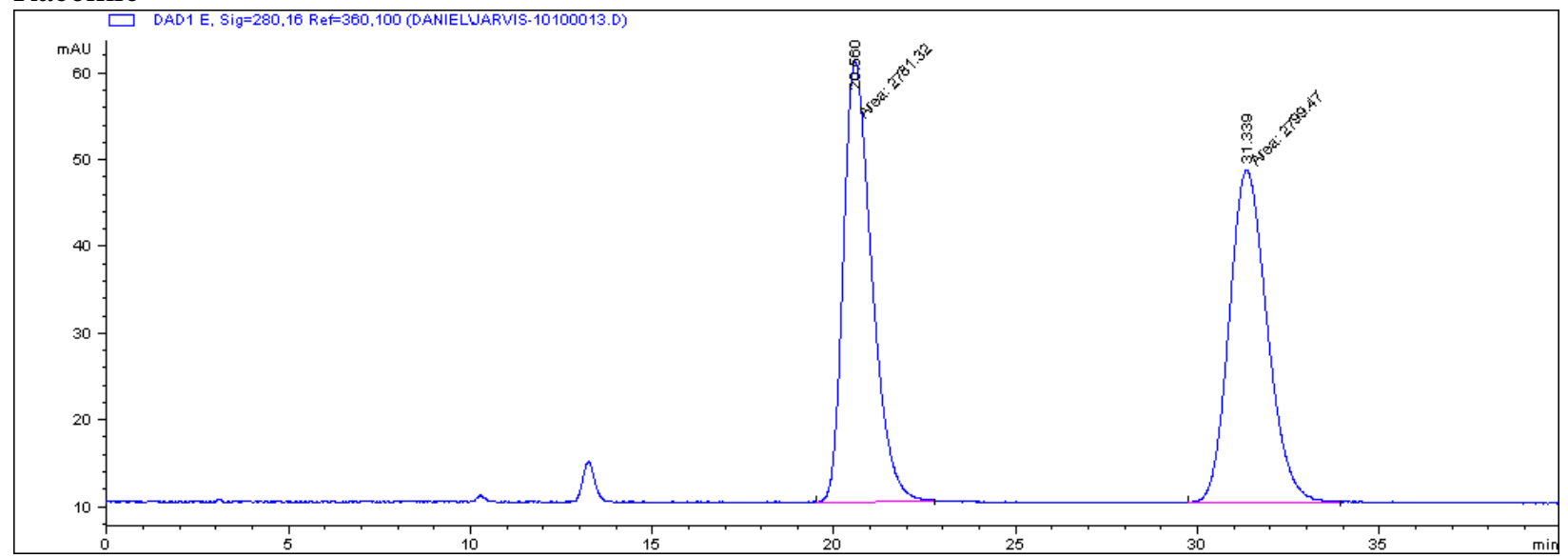


HPLC Profile of $\mathbf{3 g}$
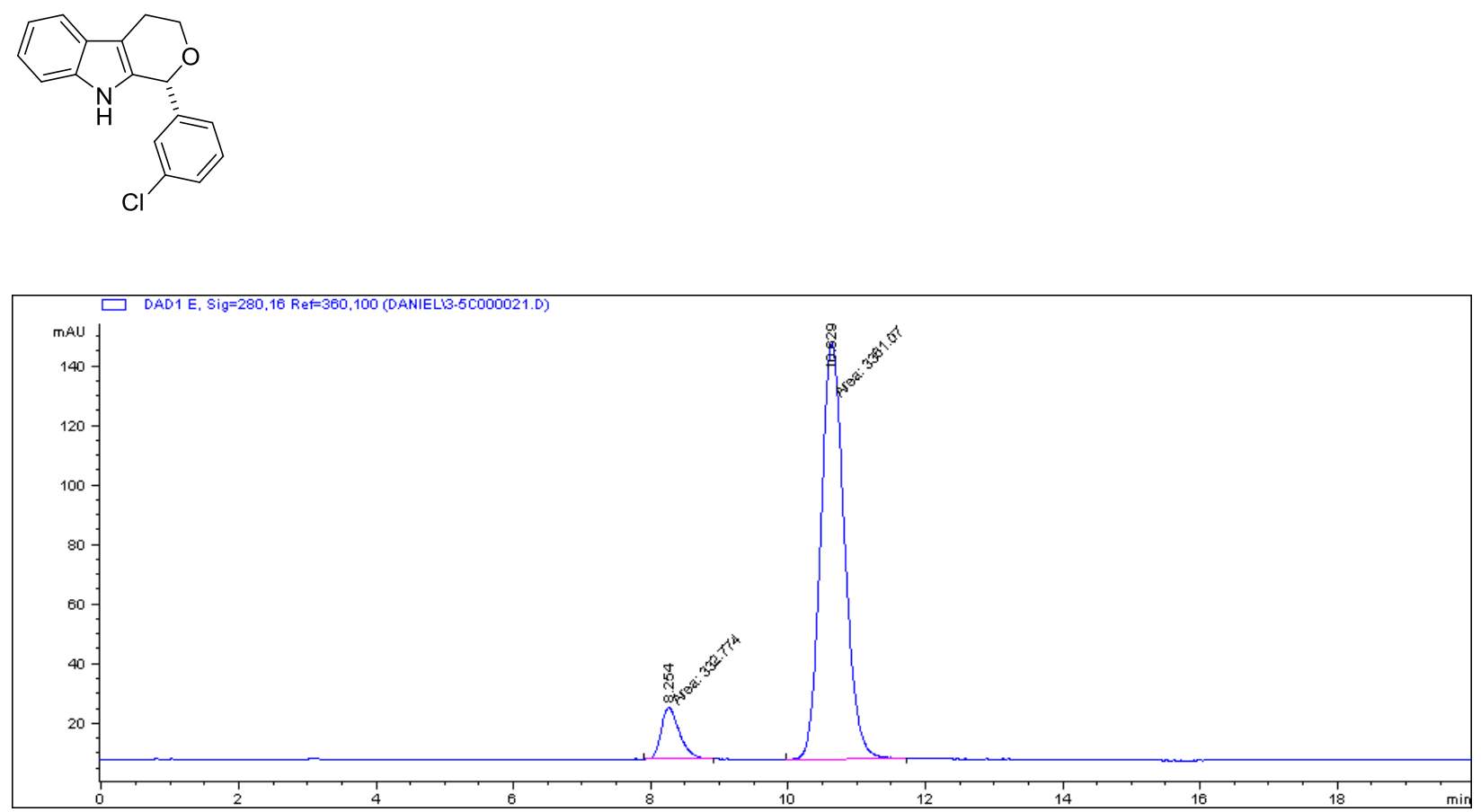

Racemic

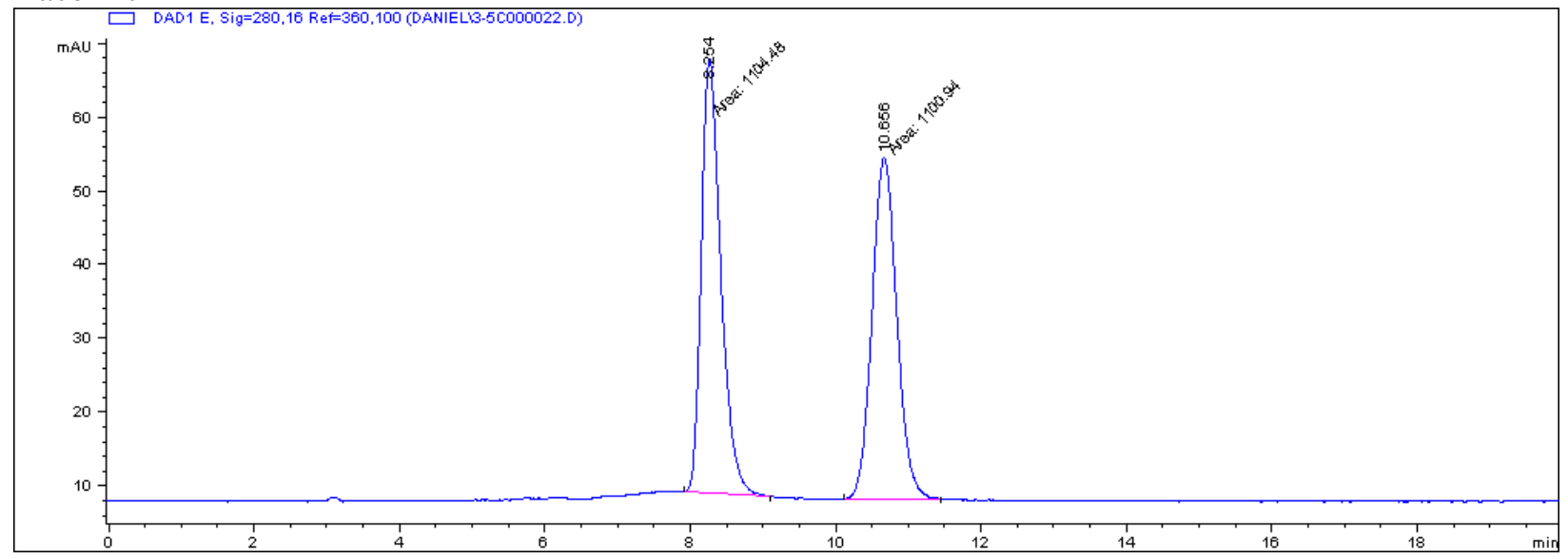


HPLC Profile of $\mathbf{3 h}$
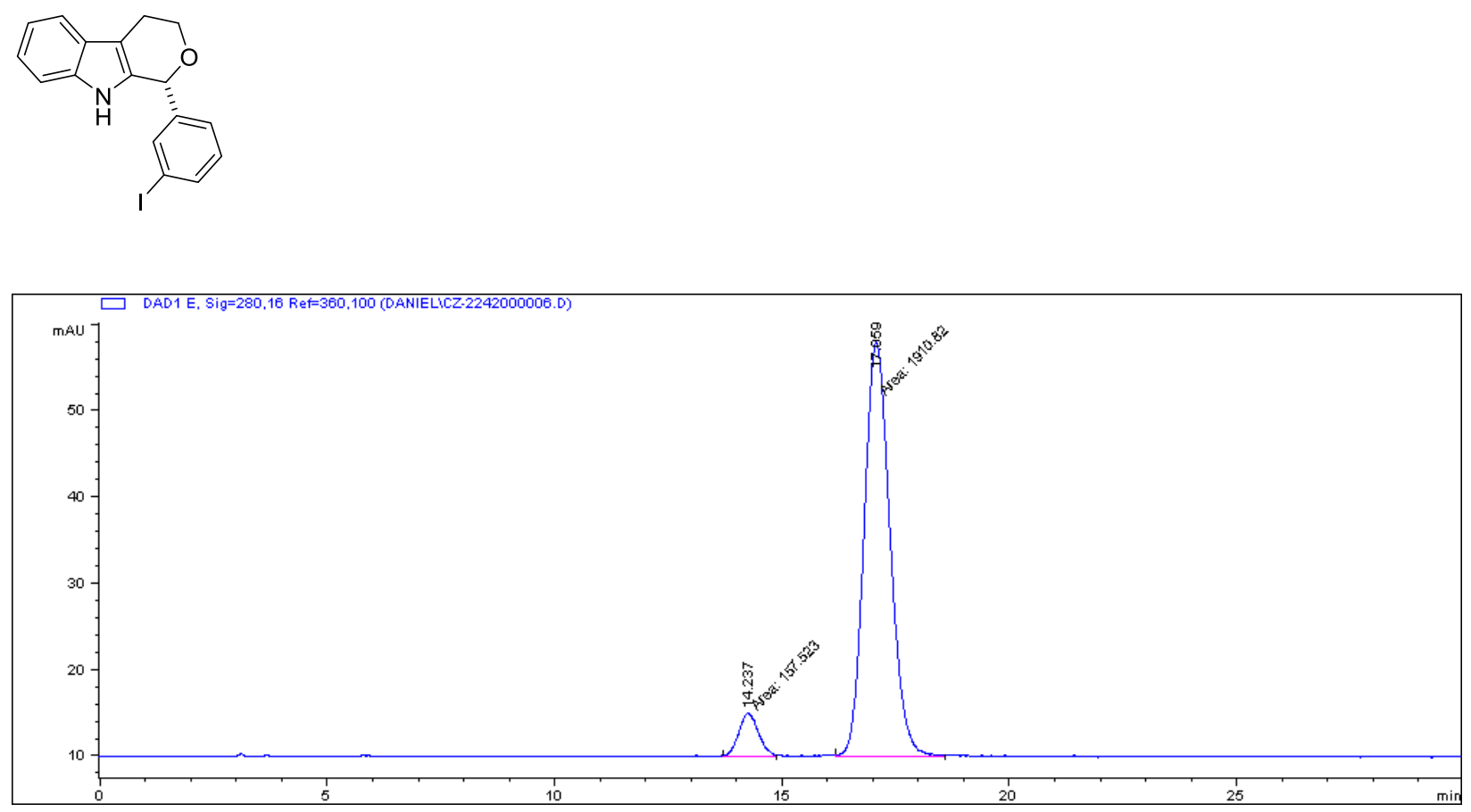

Racemic

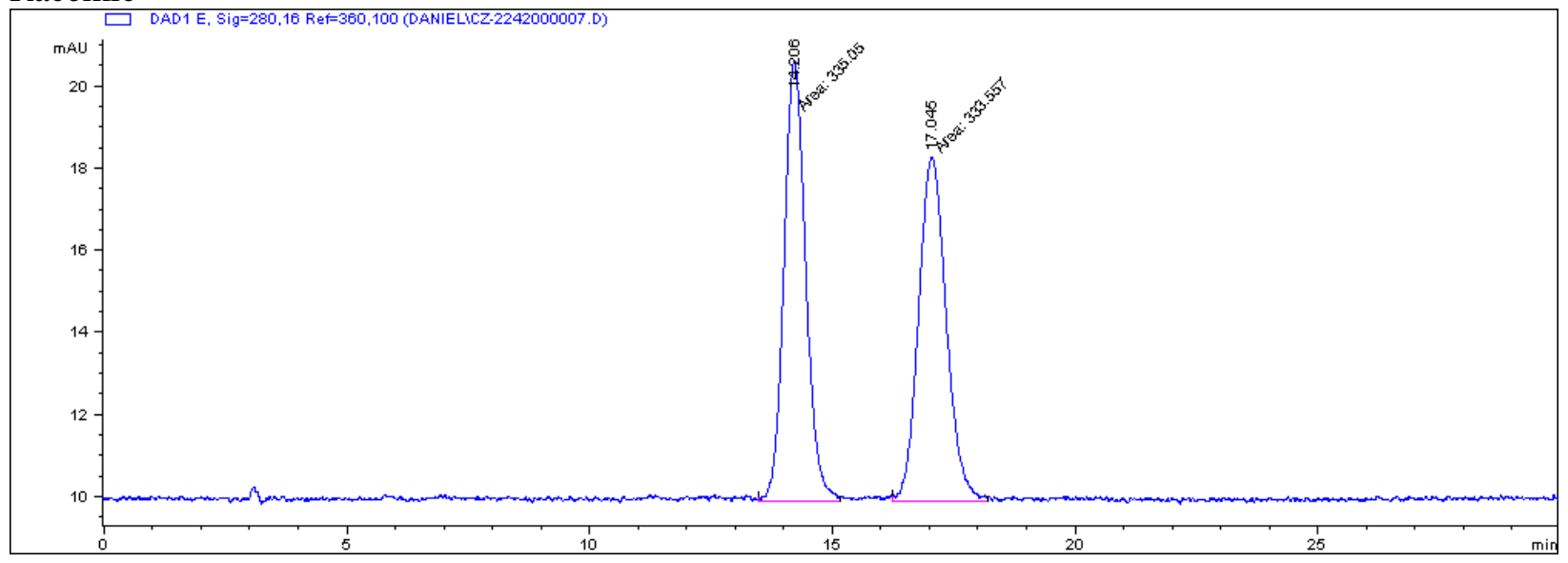


HPLC Profile of $\mathbf{3 i}$
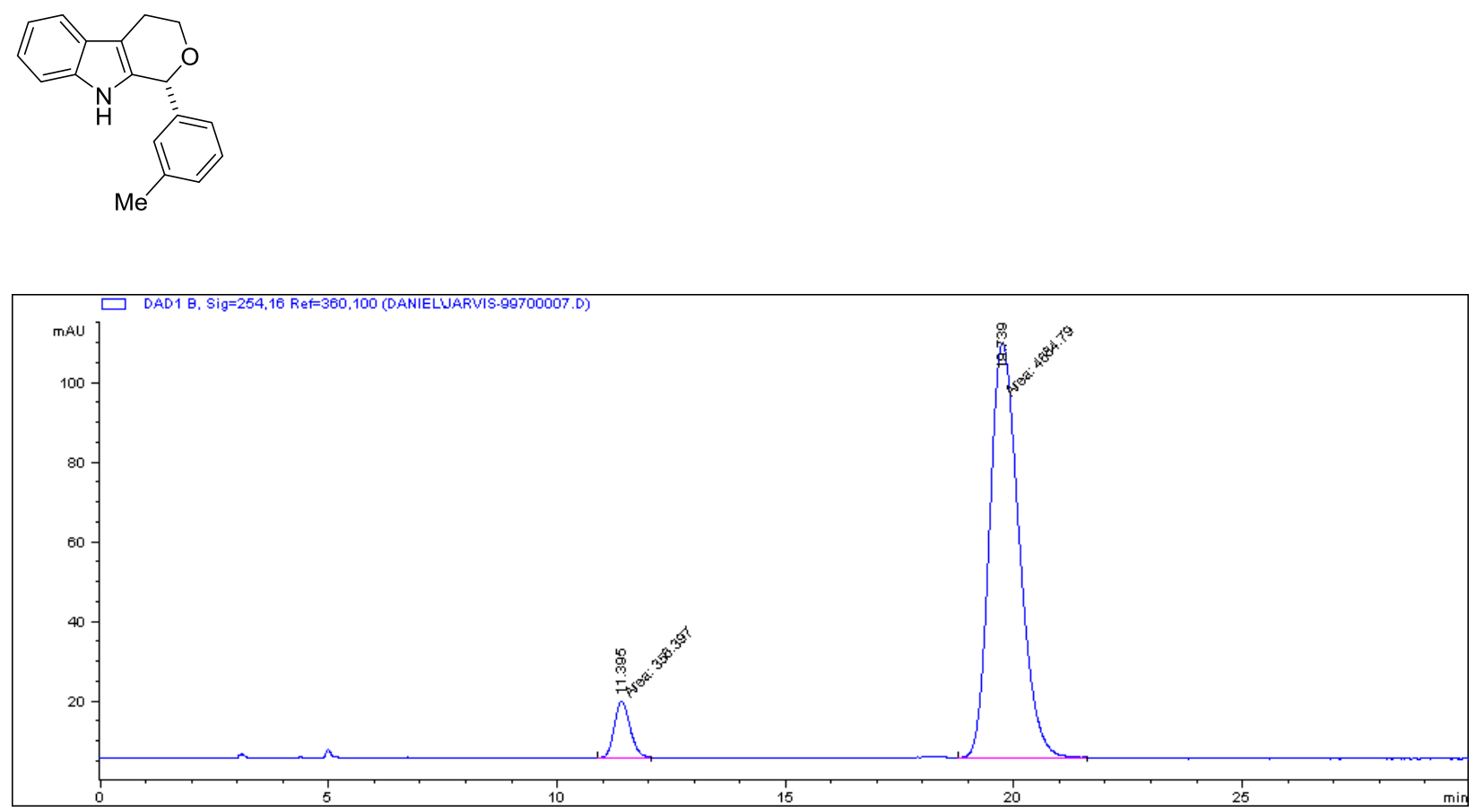

Racemic

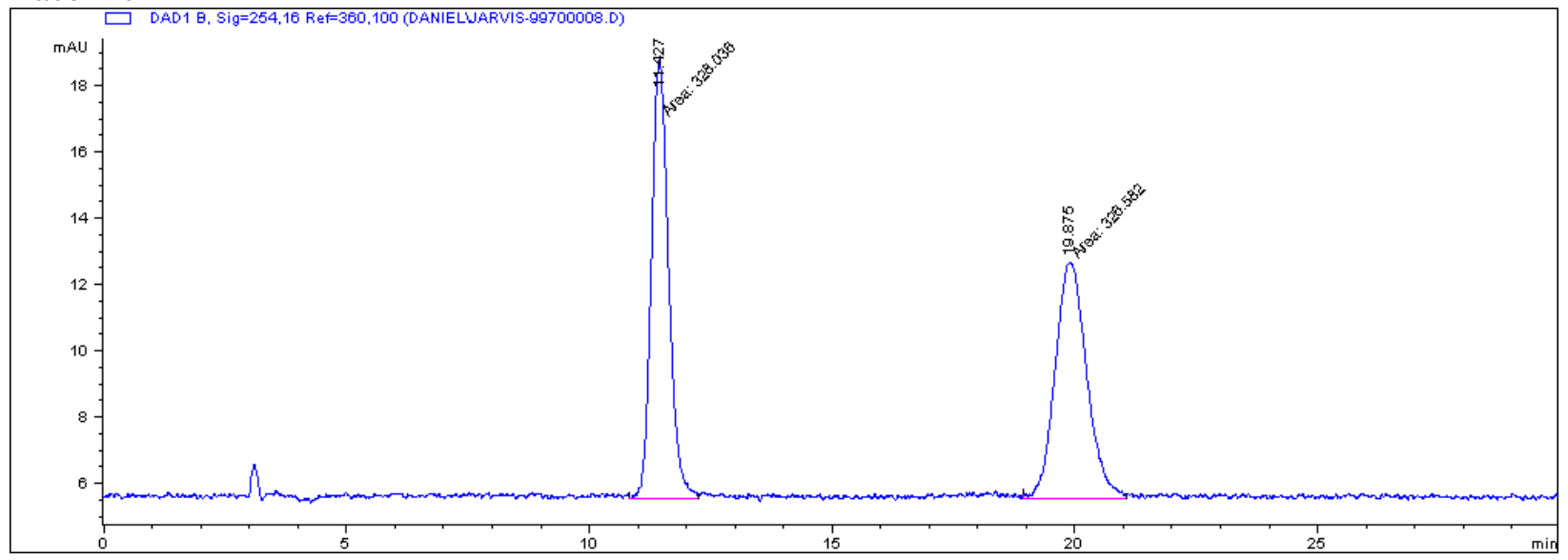


HPLC Profile of $\mathbf{3 j}$
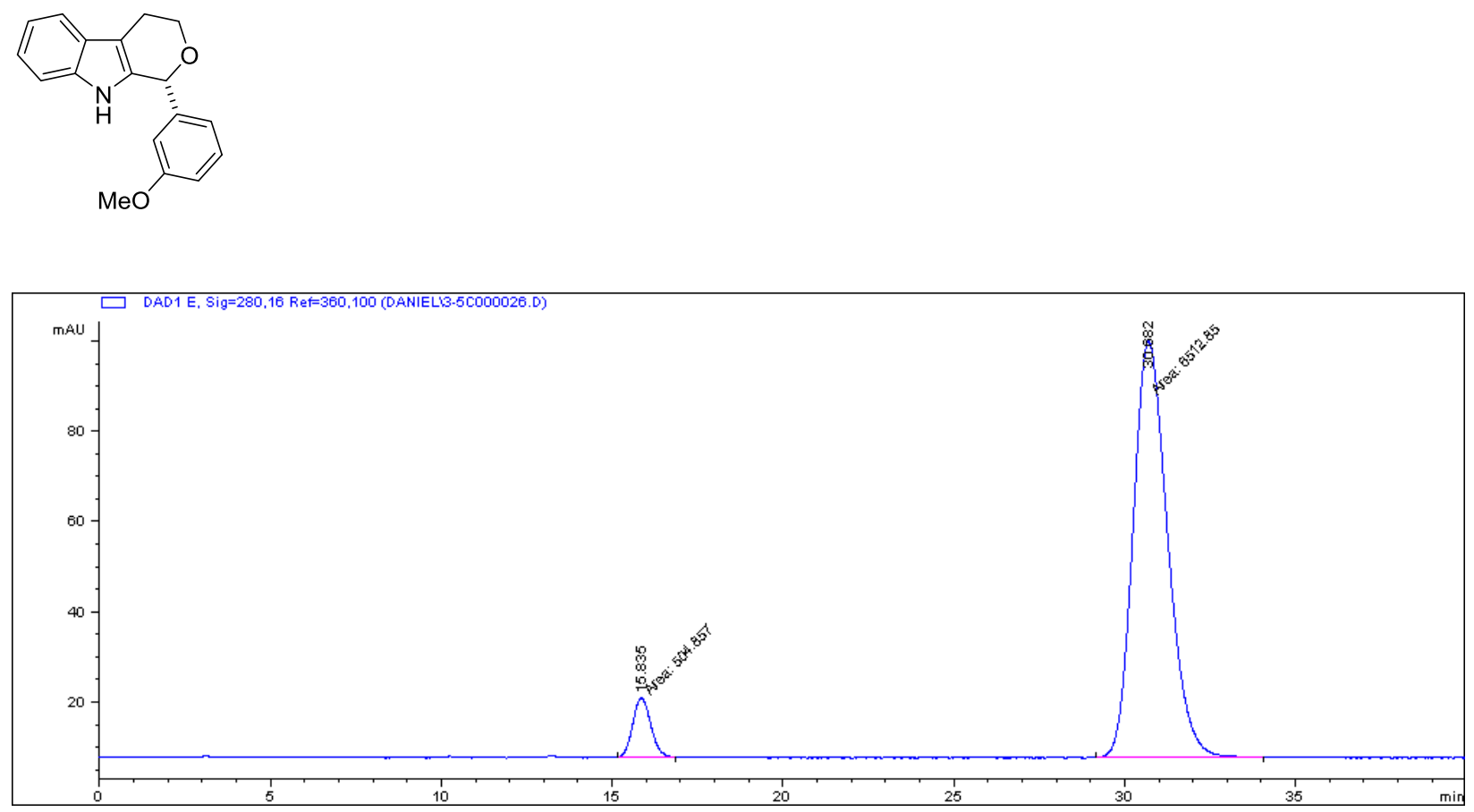

Racemic

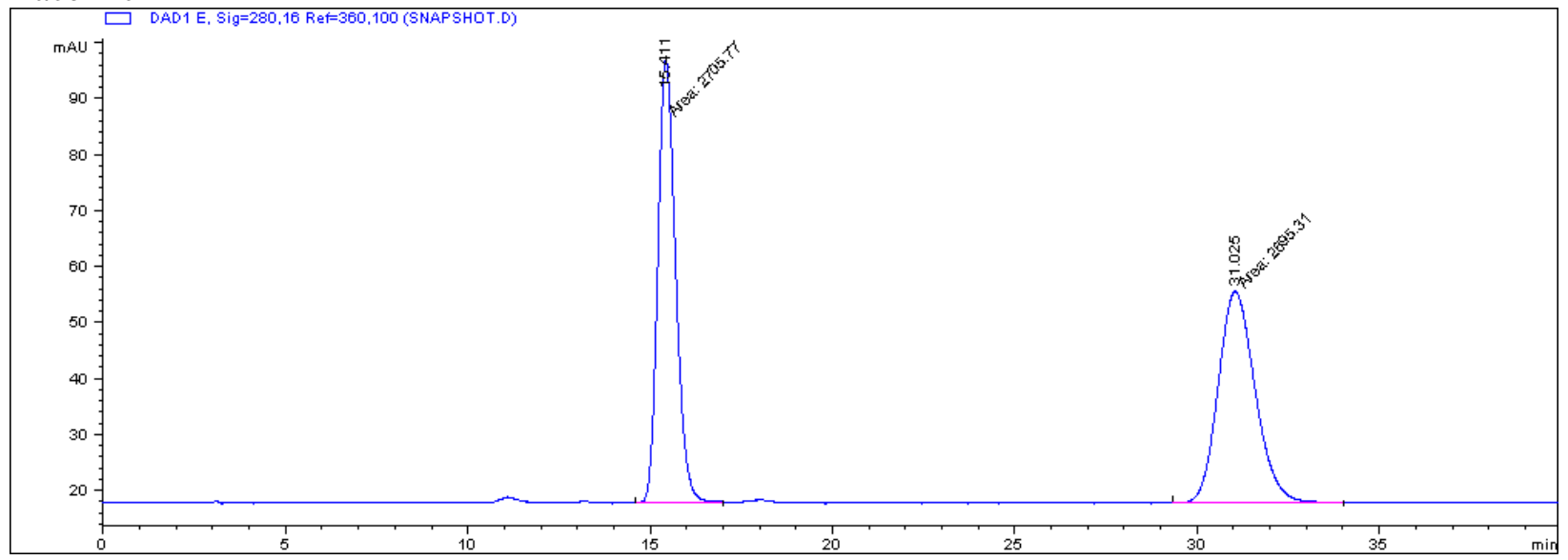


HPLC Profile of $\mathbf{3 k}$
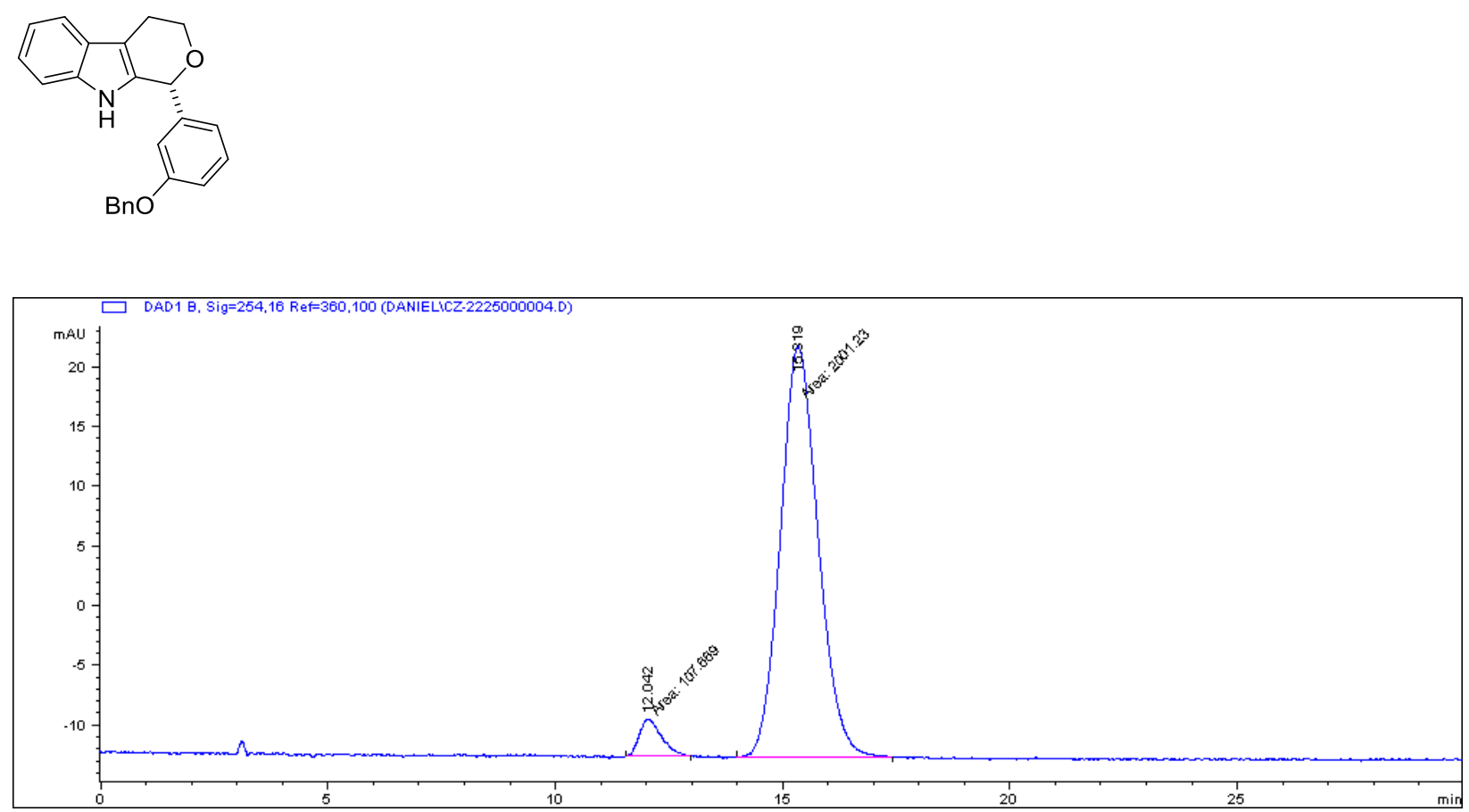

Racemic

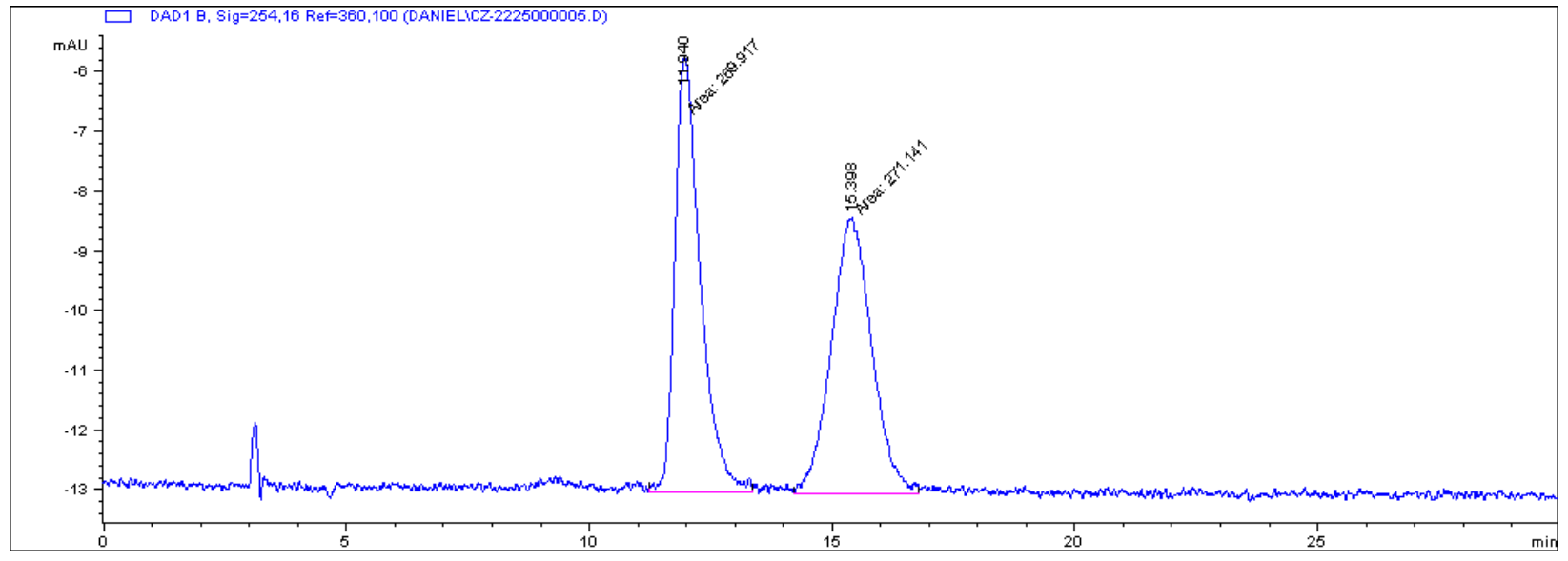


HPLC Profile of $\mathbf{3 l}$
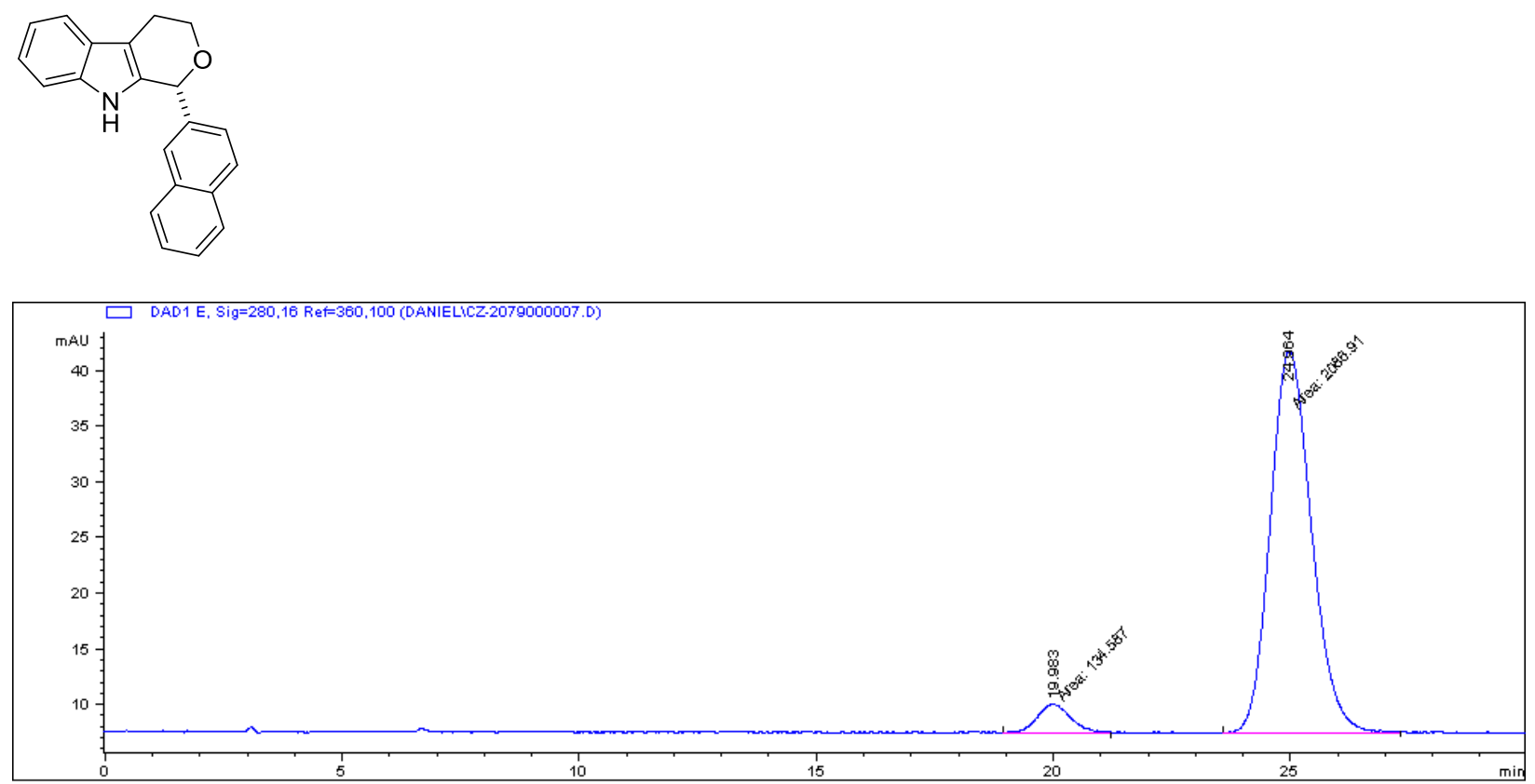

Racemic

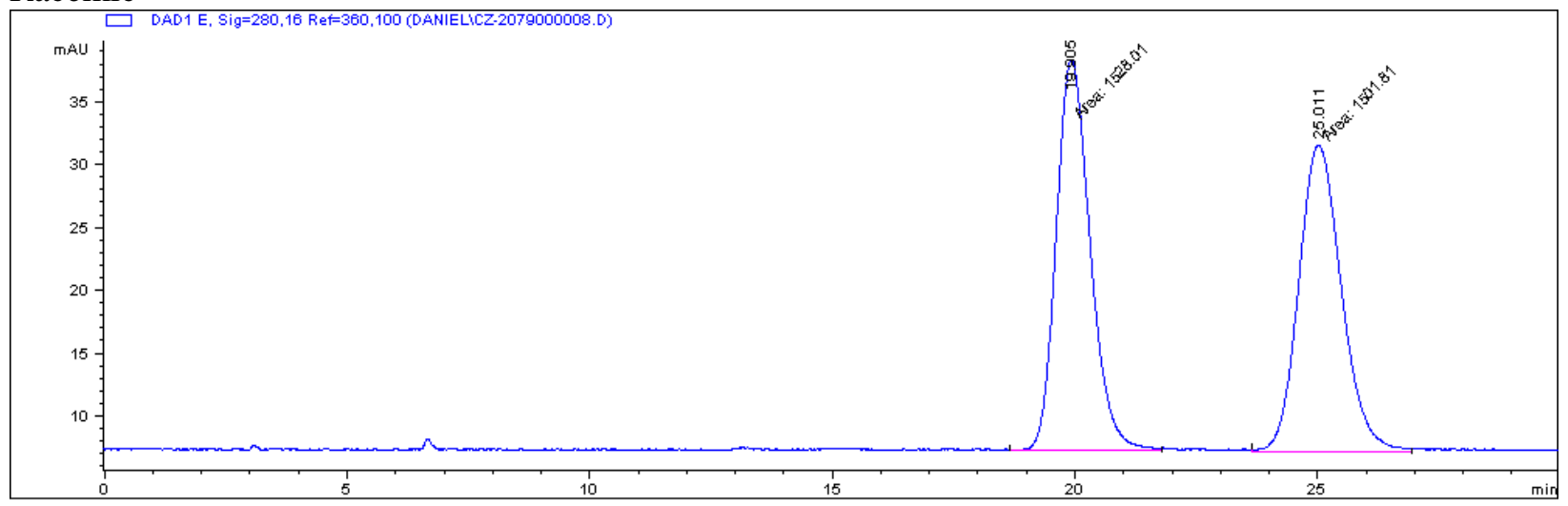


HPLC Profile of $\mathbf{3 m}$
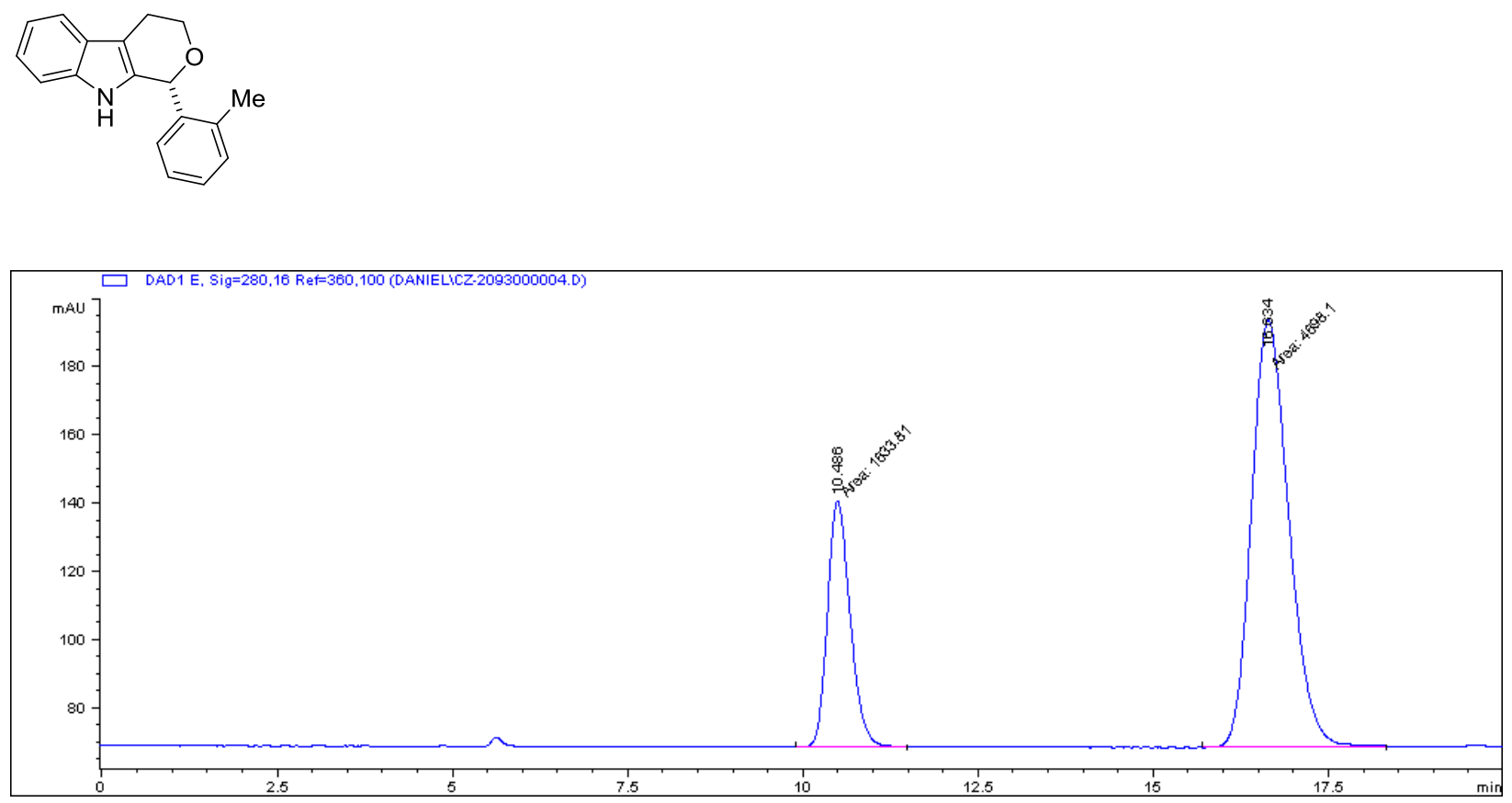

Racemic

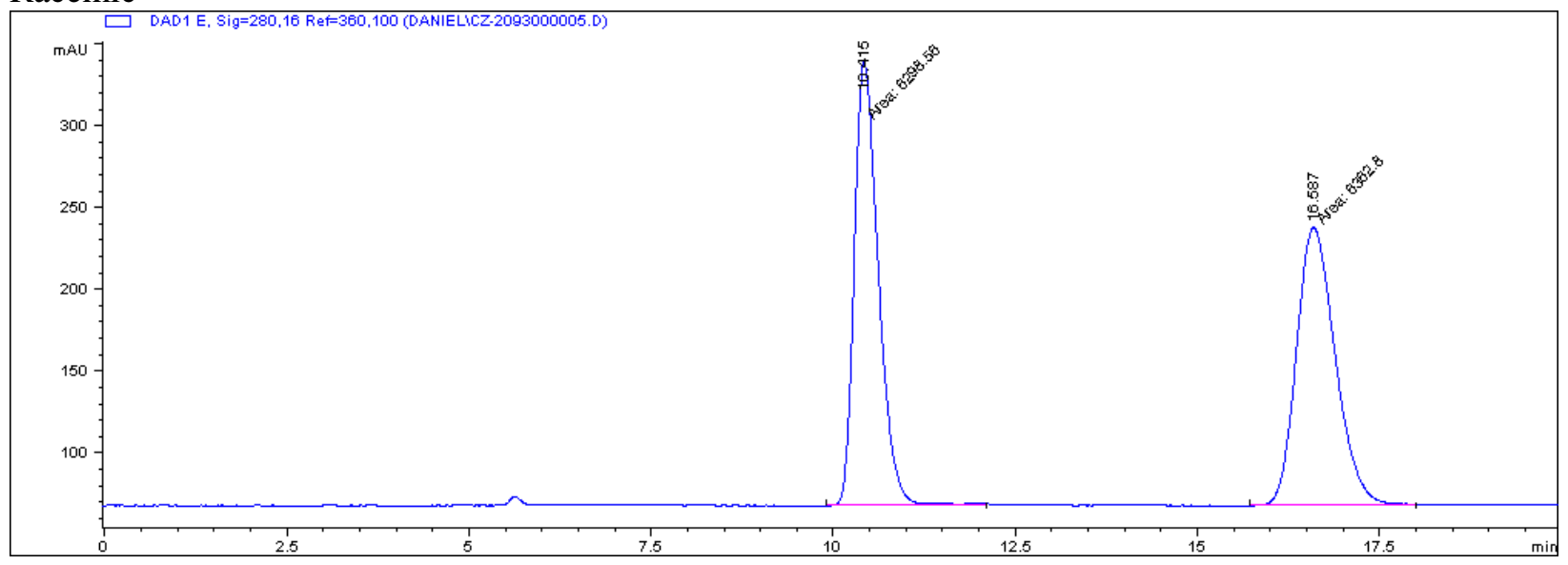


HPLC Profile of $\mathbf{3 n}$
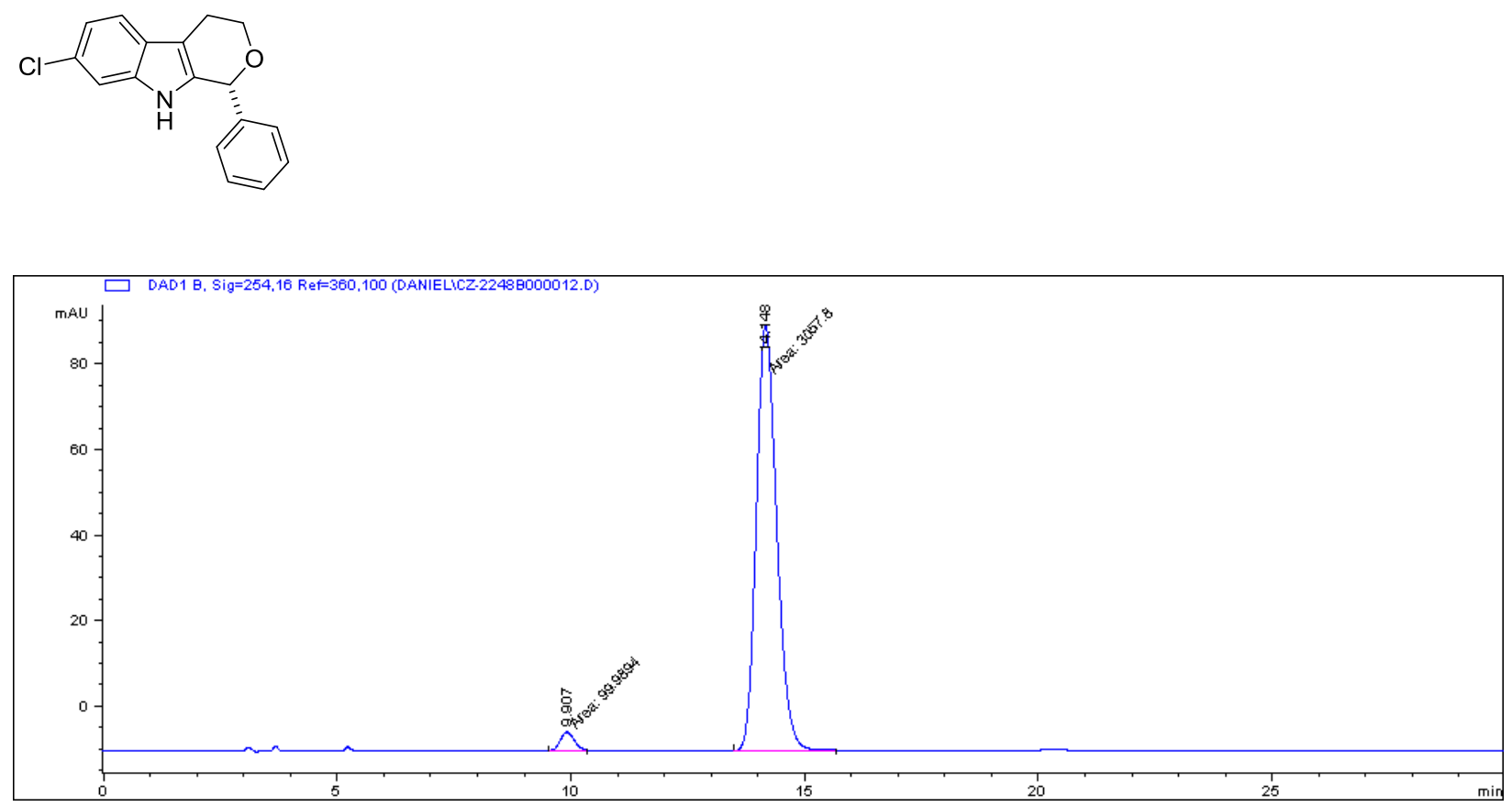

Racemic

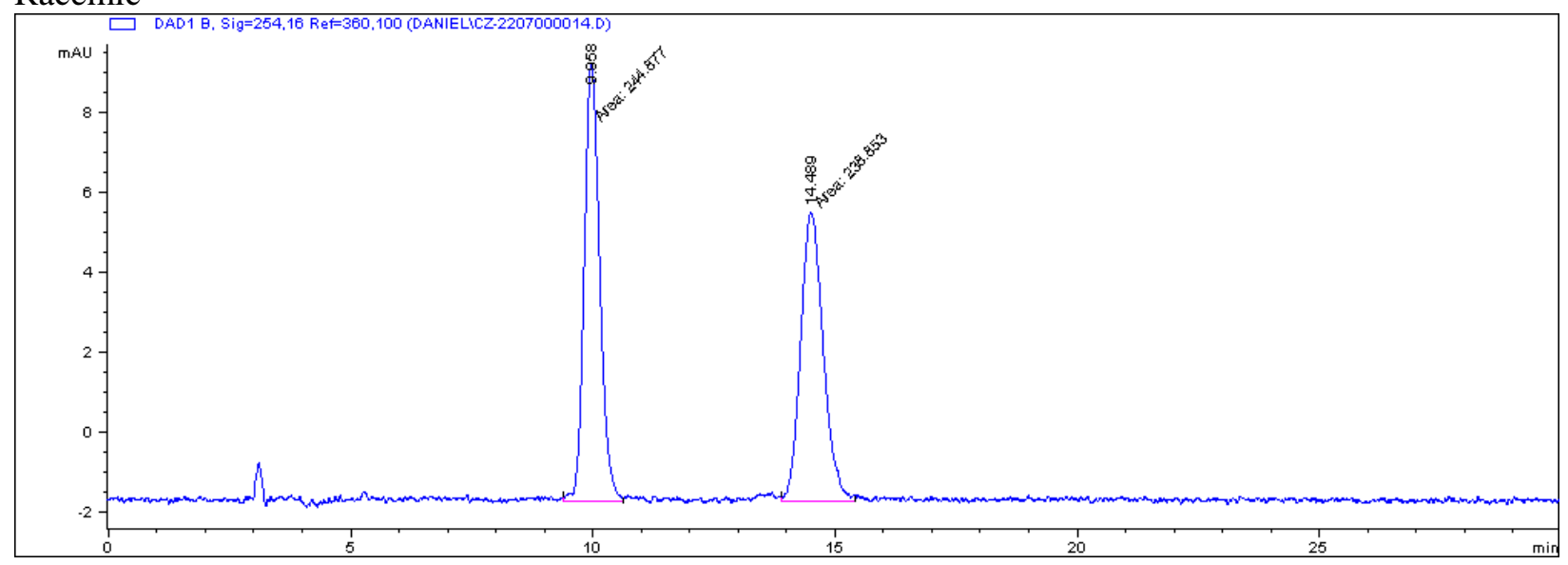




\section{HPLC Profile of $\mathbf{3 o}$}
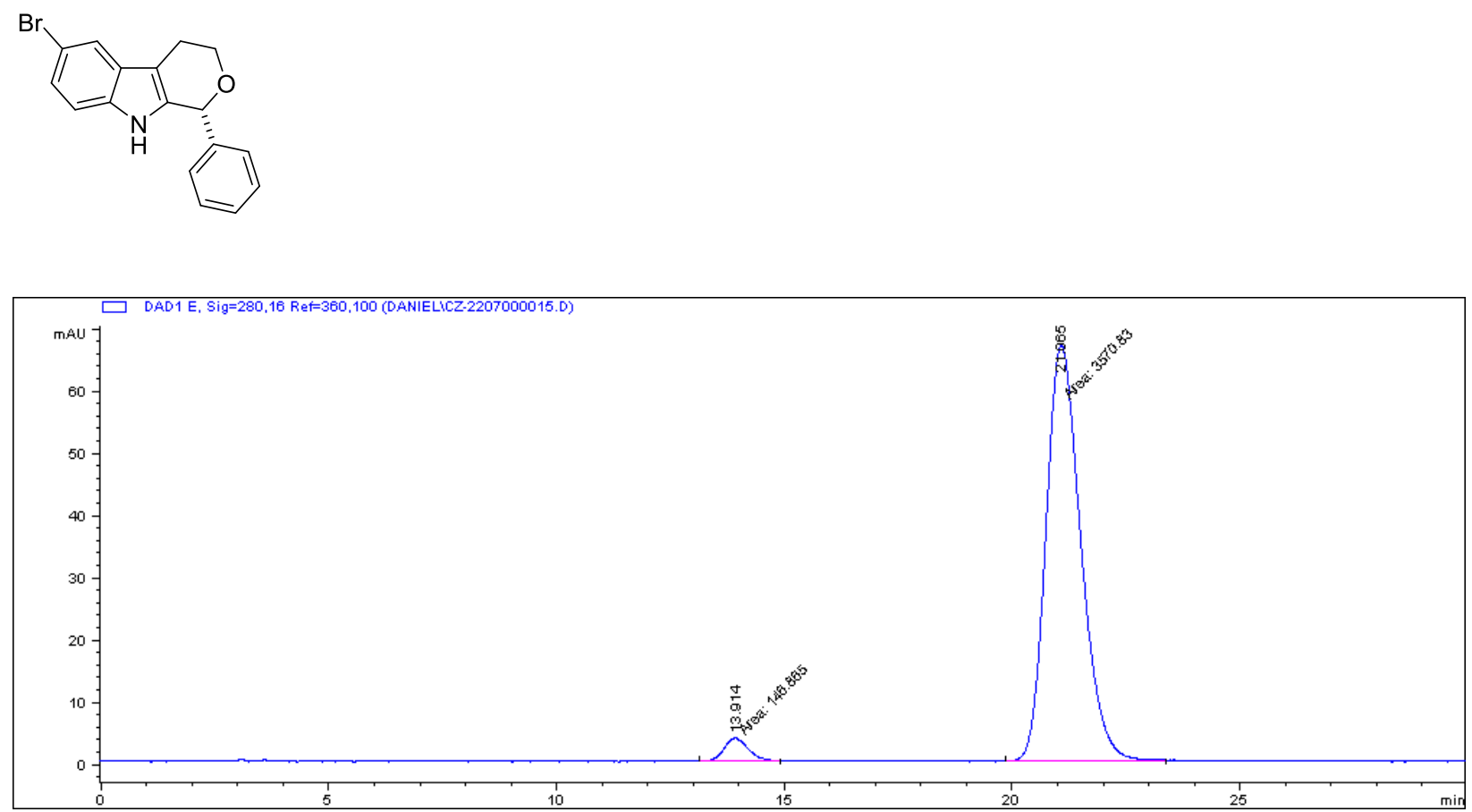

\section{Racemic}

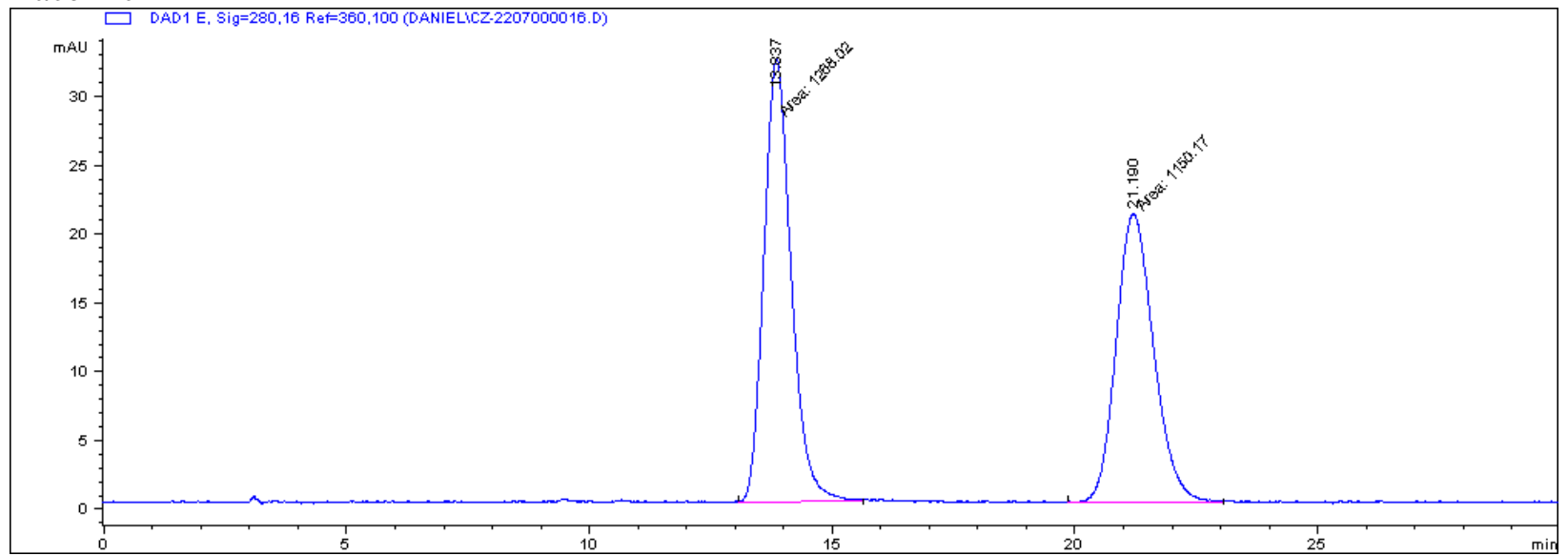




\section{HPLC Profile of $\mathbf{3 p}$}
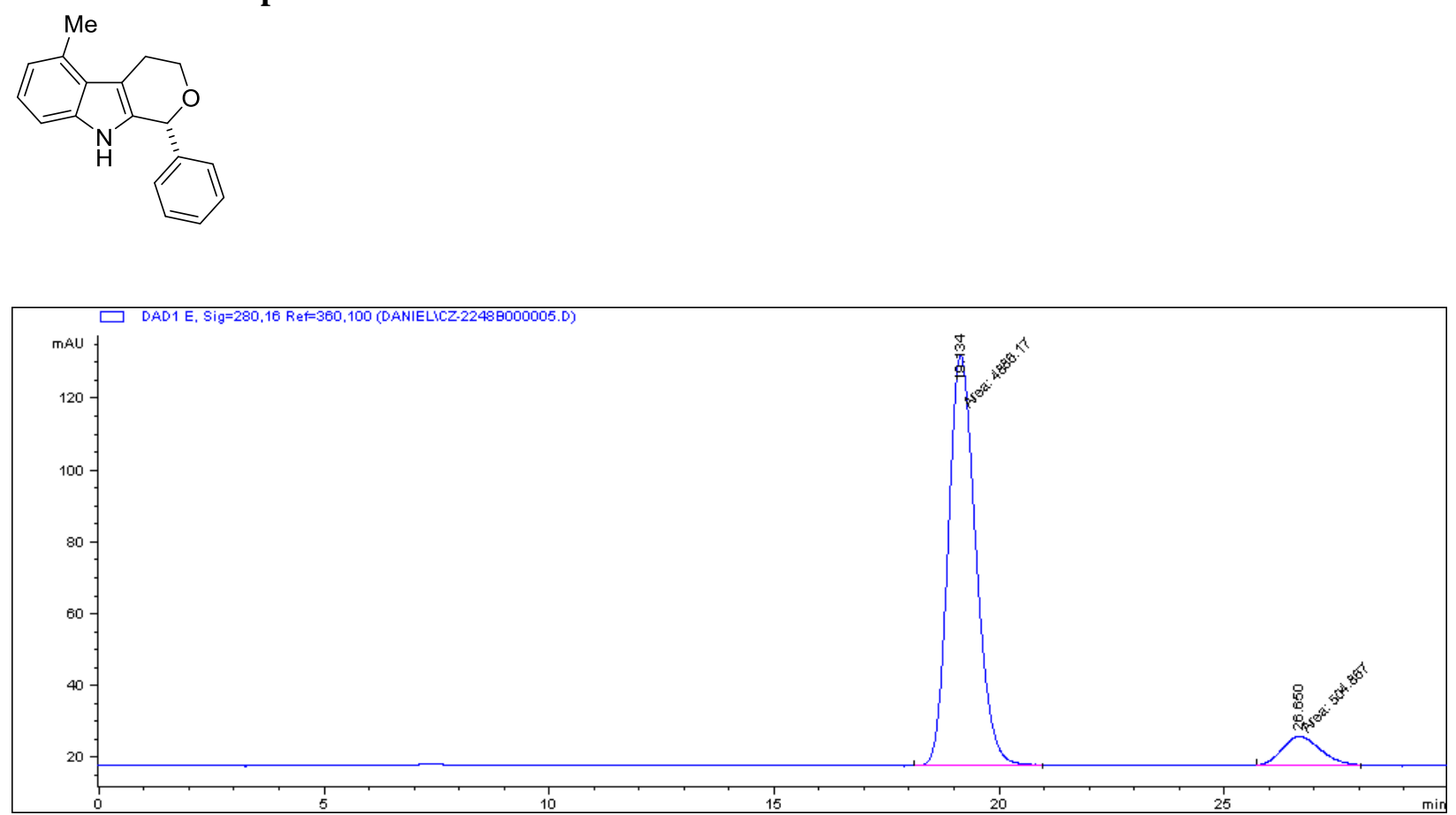

\section{Racemic}

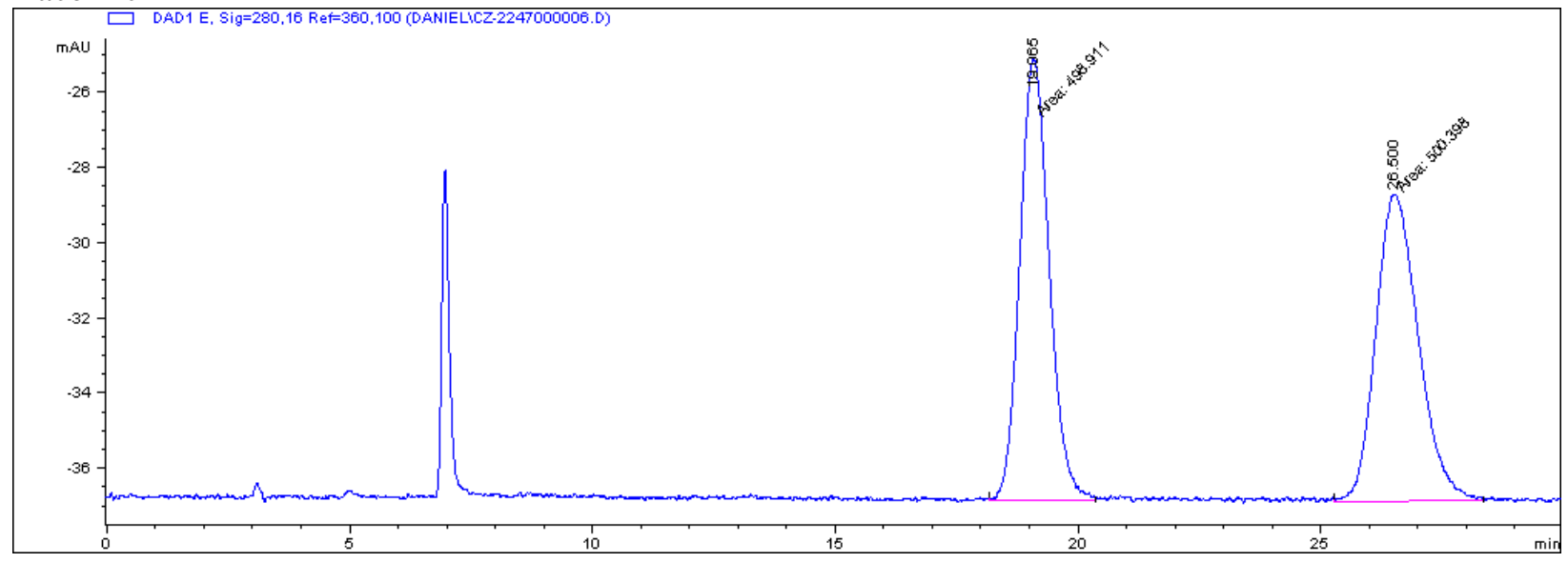


VII. NMR spectra

$$
\text { i. }
$$

${ }^{1} \mathrm{H}$ NMR of $1 \mathrm{~d} \bullet \mathrm{HCl}\left(\mathrm{CD}_{3}\right)_{2} \mathrm{SO}$

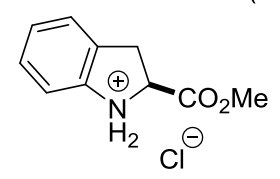

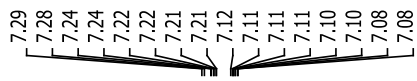

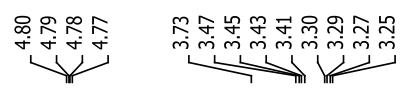

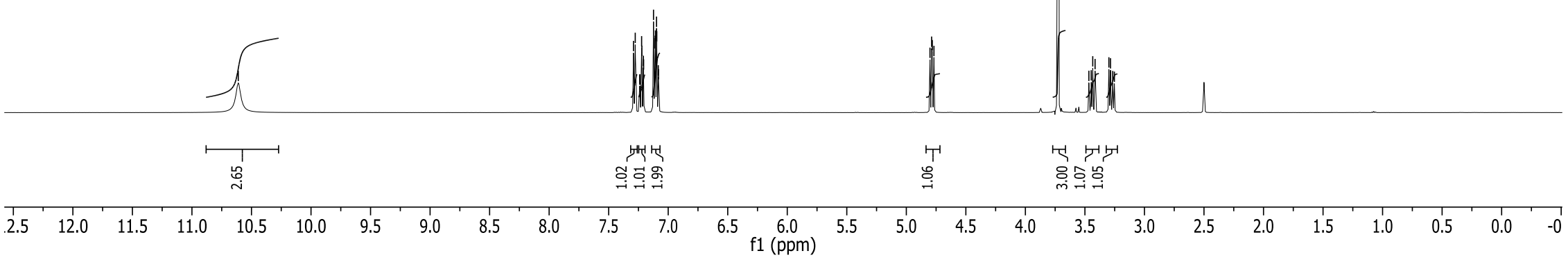


${ }^{13} \mathrm{C} \mathrm{NMR}$ of $\mathbf{1 d} \cdot \mathbf{H C l}\left(\mathrm{CD}_{3}\right)_{2} \mathrm{SO}$
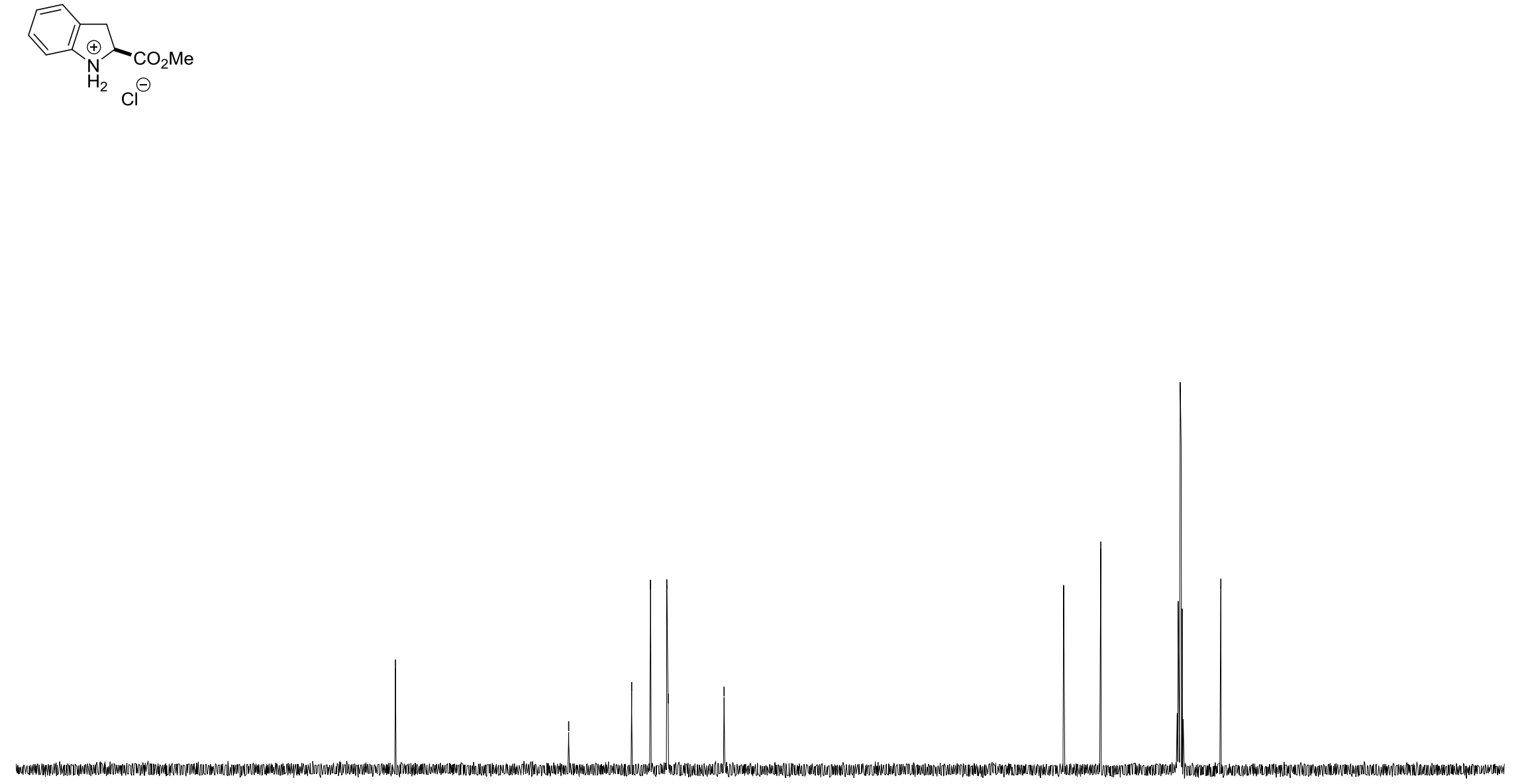

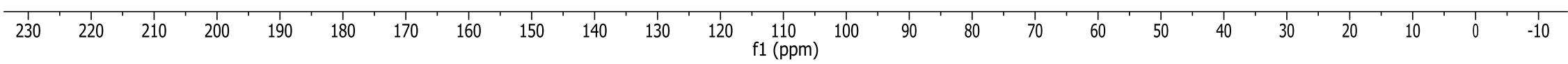




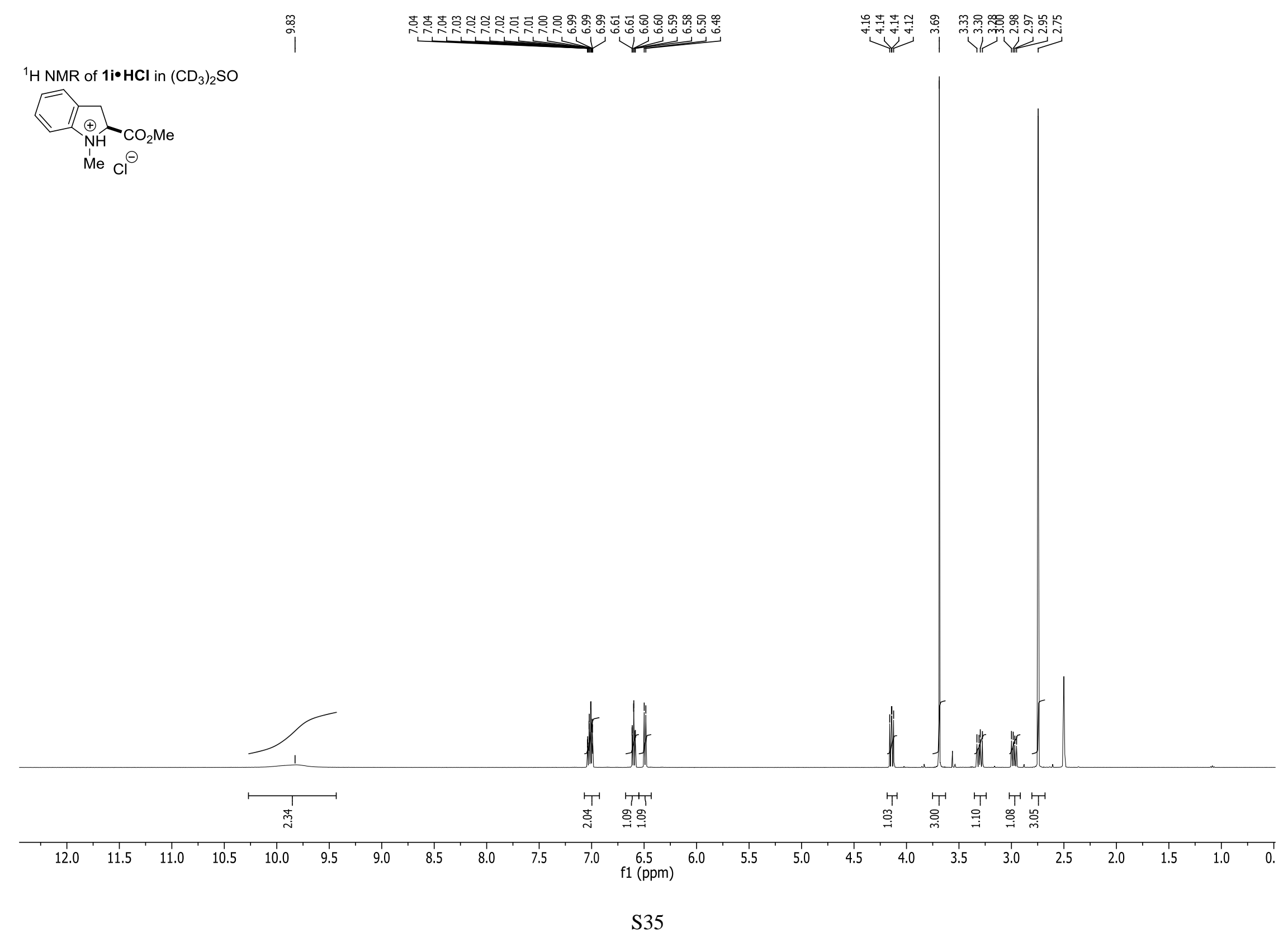


${ }^{13} \mathrm{C} \mathrm{NMR}$ of $1 \mathrm{i} \cdot \mathrm{HCl}$ in $\left(\mathrm{CD}_{3}\right)_{2} \mathrm{SO}$

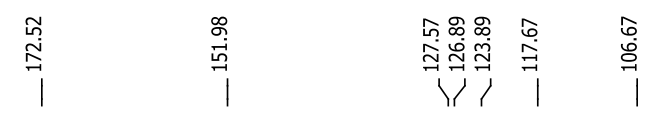

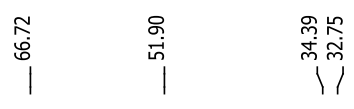

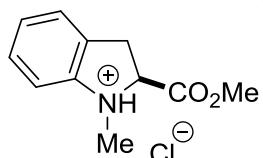

$\mathrm{Me} \mathrm{Cl}^{\ominus}$

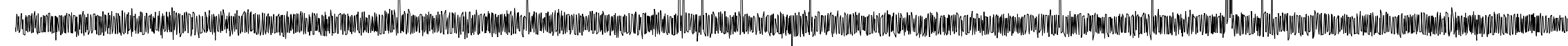

\begin{tabular}{|c|c|c|c|c|c|c|c|c|c|c|c|c|c|c|c|c|c|c|c|c|c|c|c|}
\hline 230 & 220 & 210 & 200 & 190 & 180 & 170 & 160 & 150 & 140 & 130 & 120 & $\begin{array}{c}110 \\
\mathrm{f} 1(\mathrm{ppm})\end{array}$ & 100 & 90 & 80 & 70 & 60 & 50 & 40 & 30 & 20 & 10 & -10 \\
\hline
\end{tabular}




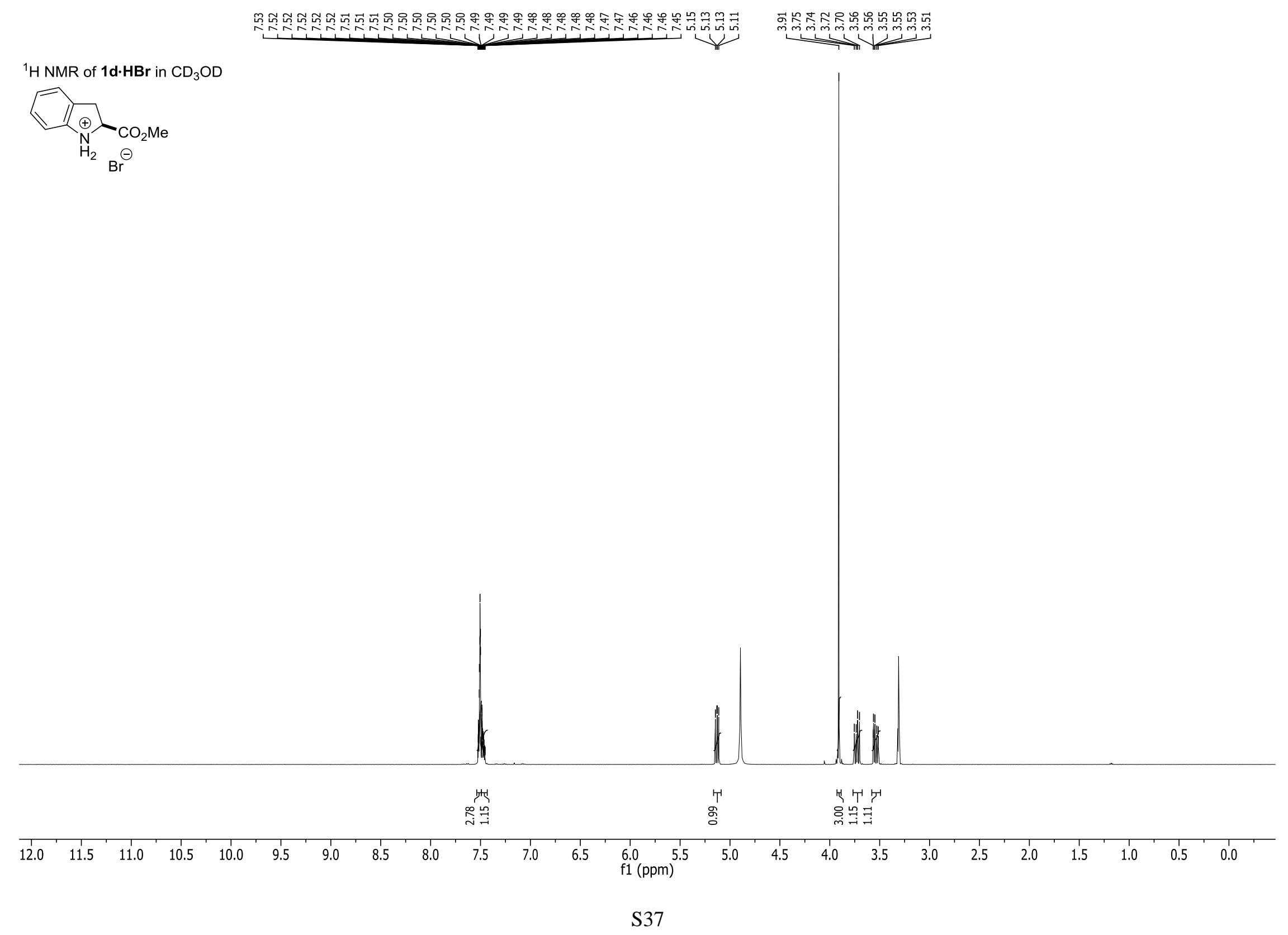



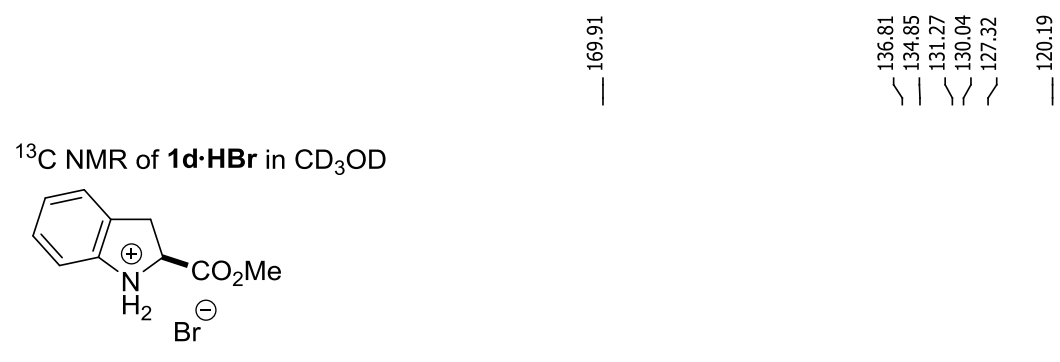

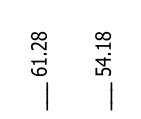

$\stackrel{m}{\stackrel{m}{m}}$

\begin{tabular}{llllllllllllllllllllllllllllll}
\hline 230 & 220 & 210 & 200 & 190 & 180 & 170 & 160 & 150 & 140 & 130 & 120 & 110 & 100 & 90 & 80 & 70 & 60 & 50 & 40 & 30 & 20 & 10 & 0 & -10
\end{tabular}

S38 


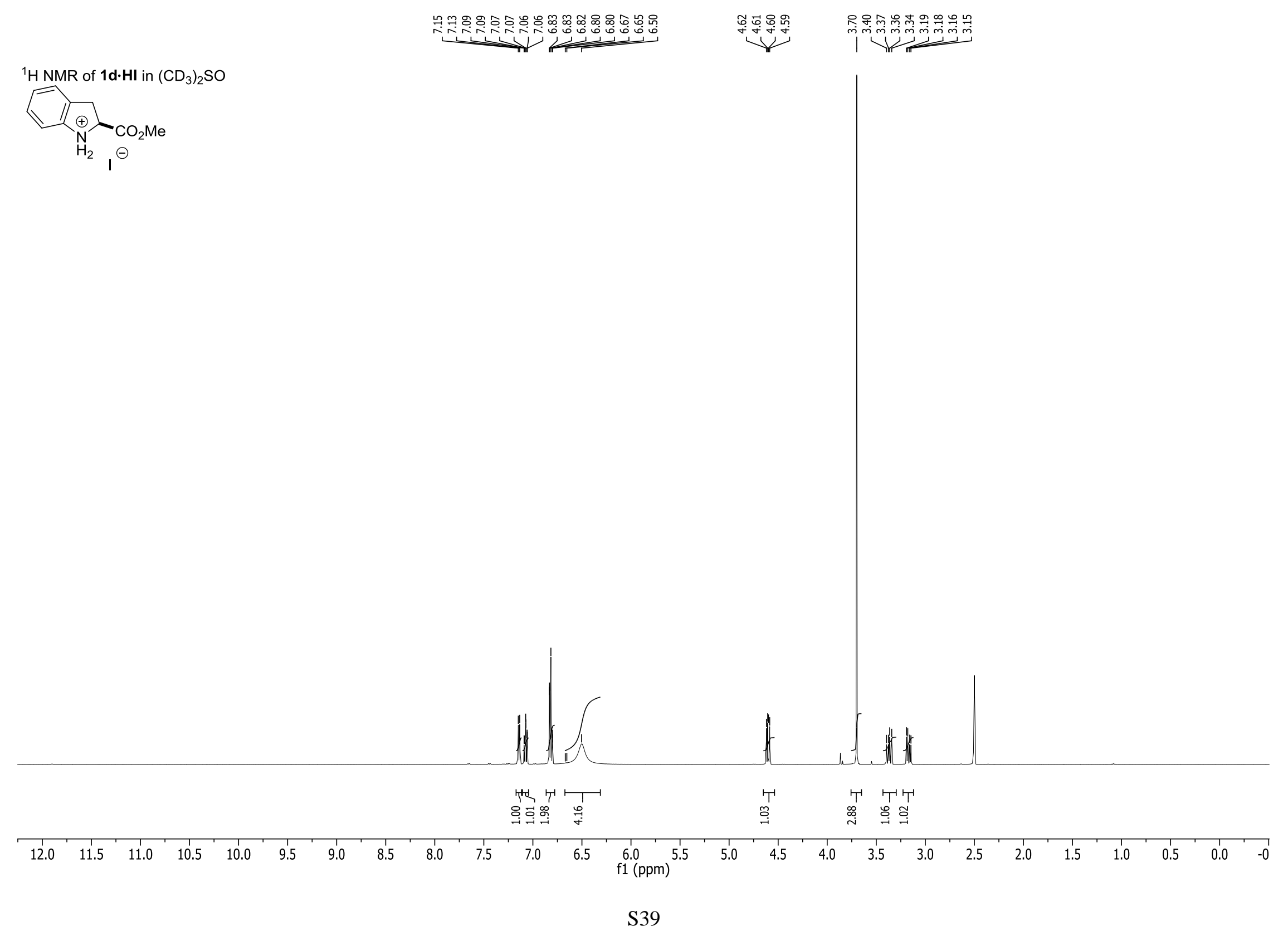




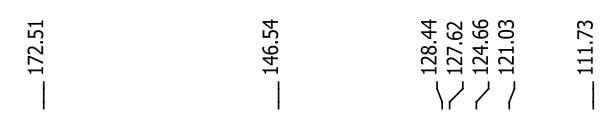

${ }^{13} \mathrm{C} \mathrm{NMR}$ of $\mathbf{1 d} \cdot \mathrm{HI}$ in $\left(\mathrm{CD}_{3}\right)_{2} \mathrm{SO}$

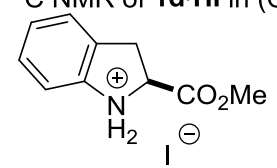

${ }^{\ominus}$

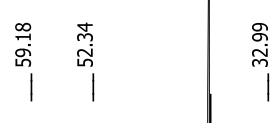

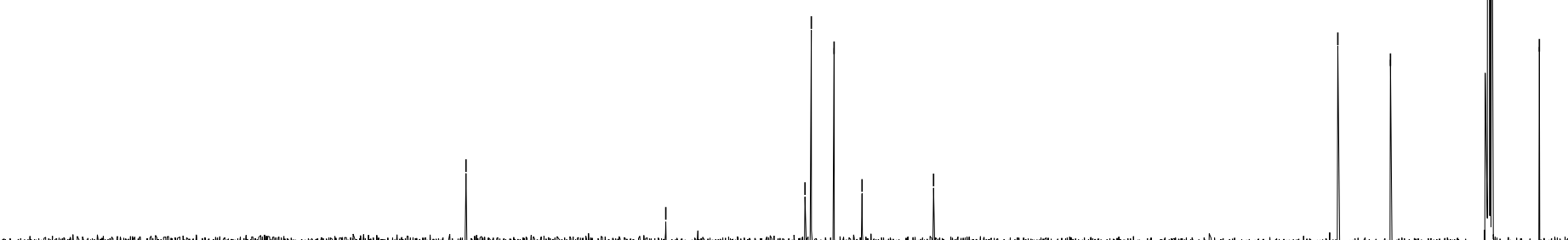

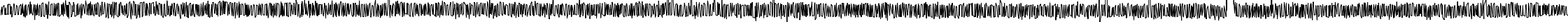

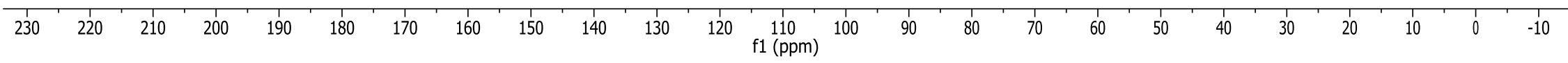




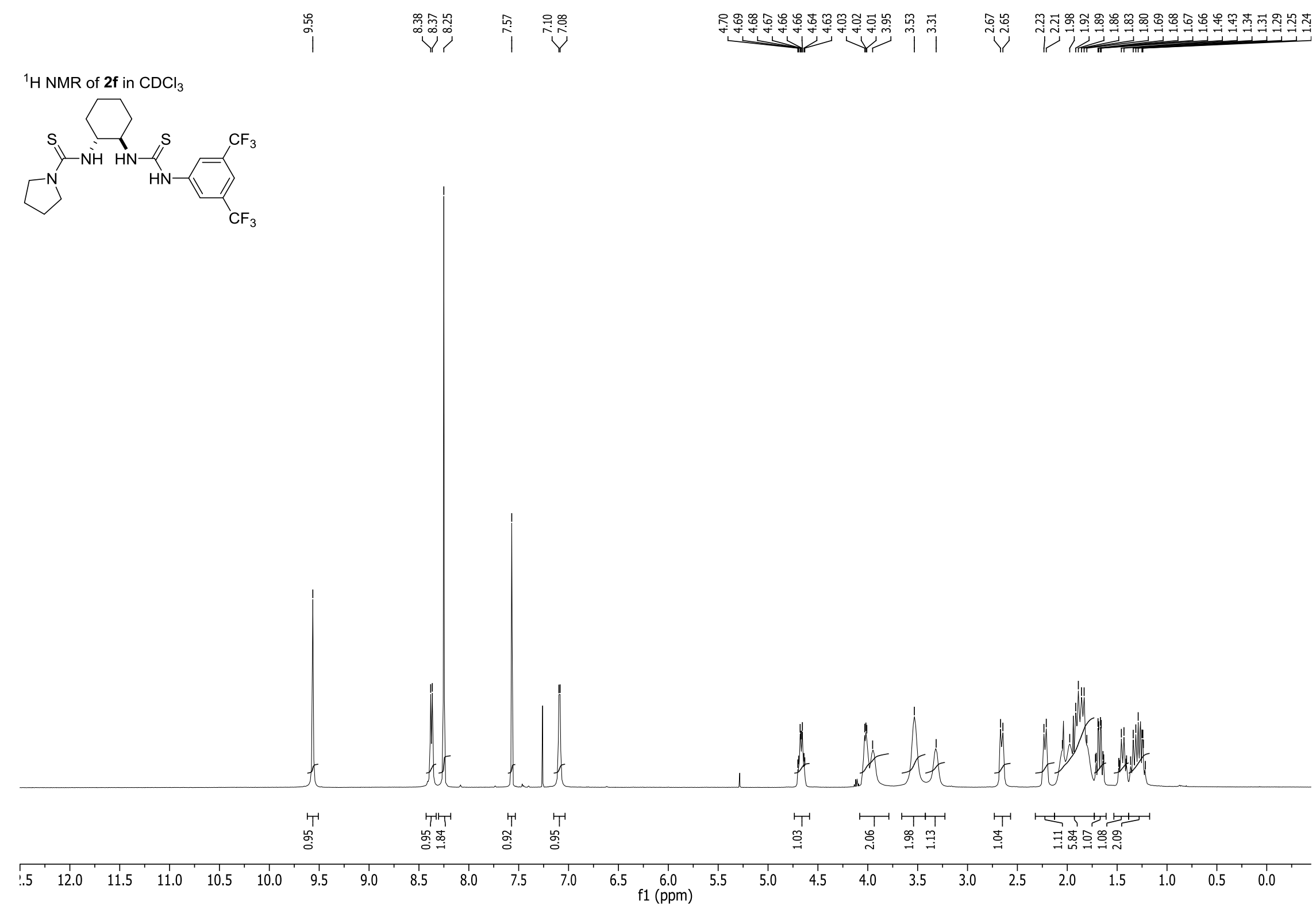




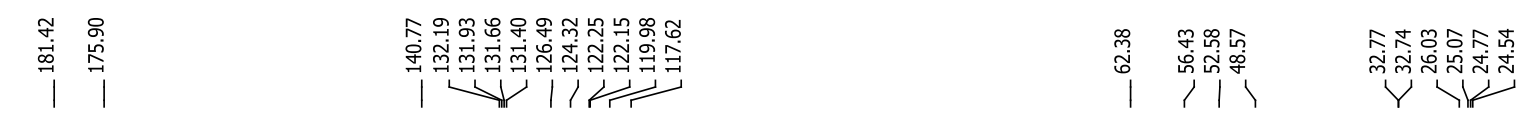

${ }^{13} \mathrm{C} \mathrm{NMR}$ of $\mathbf{2} \mathbf{f}$ in $\mathrm{CDCl}_{3}$
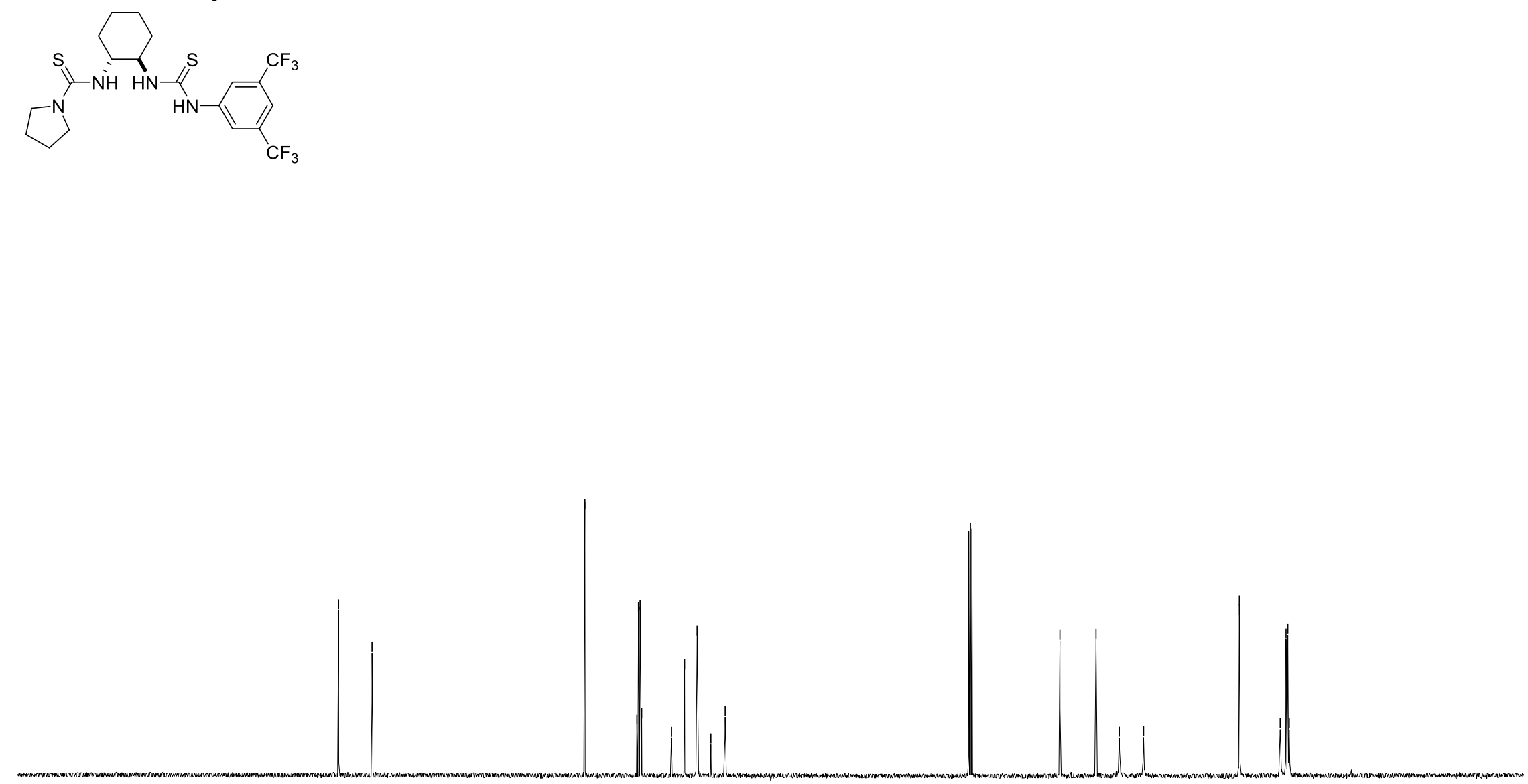

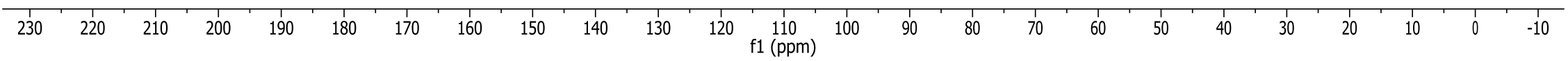


${ }^{1} \mathrm{H}$ NMR of $\mathbf{2 g}$ in $\mathrm{CDCl}_{3}$

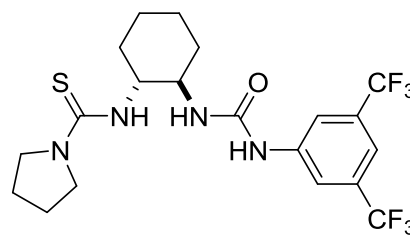

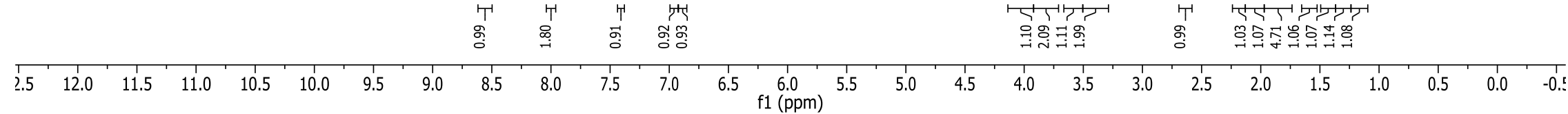




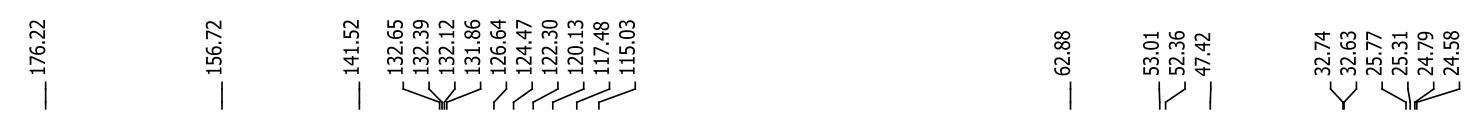

${ }^{13} \mathrm{C} \mathrm{NMR}$ of $\mathbf{2} \mathbf{g}$ in $\mathrm{CDCl}_{3}$
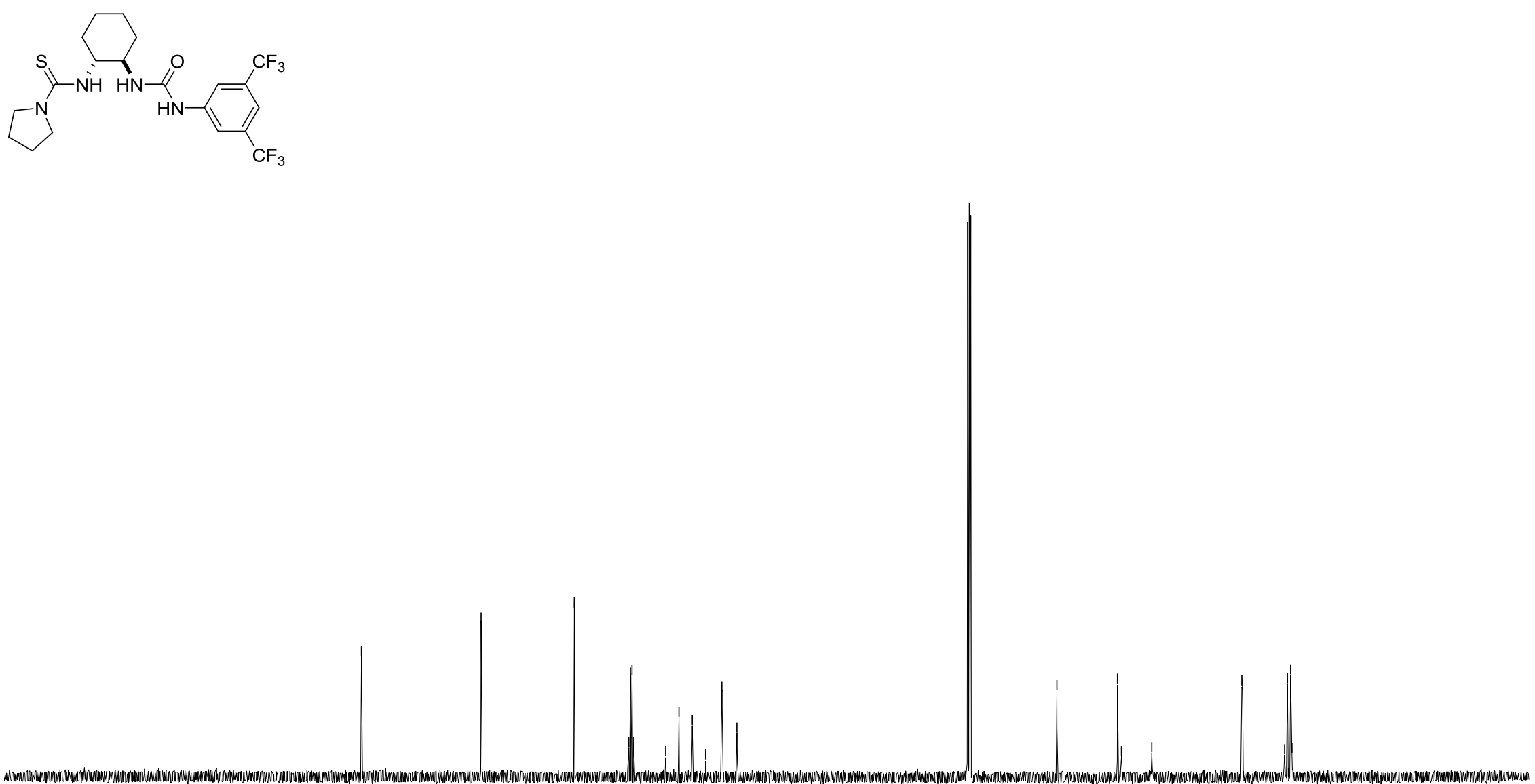

\begin{tabular}{lllllllllllllllllllllllll}
\hline 230 & 220 & 210 & 200 & 190 & 180 & 170 & 160 & 150 & 140 & 130 & 120 & $\begin{array}{l}1 \\
\mathrm{f} 1(\mathrm{ppm})\end{array}$ & 100 & 90 & 80 & 70 & 60 & 50 & 40 & 30 & 20 & 10 & 0 & -10
\end{tabular}


${ }^{1} \mathrm{H}$ NMR of $\mathbf{2 h}$ in $\mathrm{CDCl}_{3}$

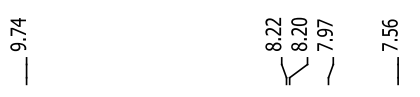

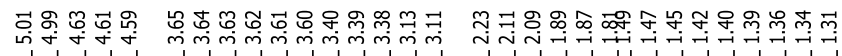<smiles>O=C(NC1CCCCC1NC(=S)Nc1cc(C(F)(F)F)cc(C(F)(F)F)c1)N1CCCC1</smiles>

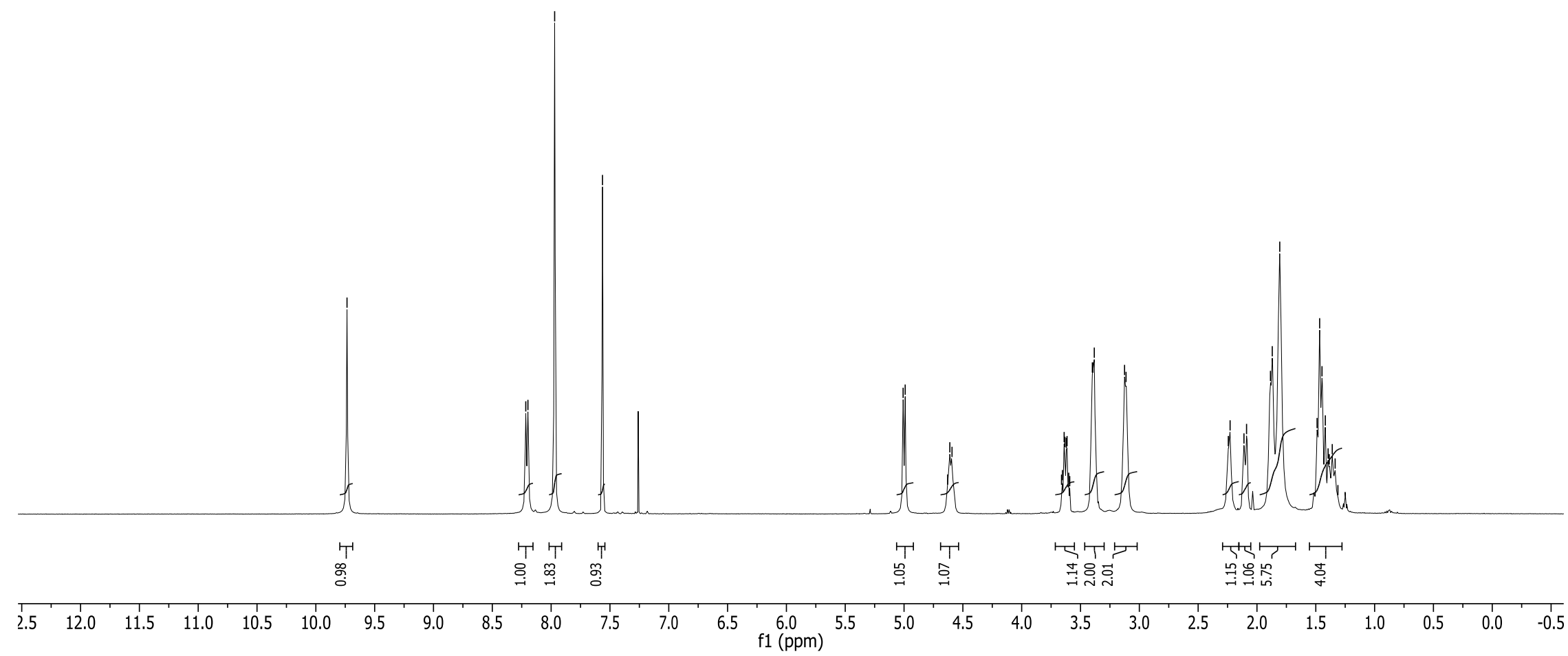



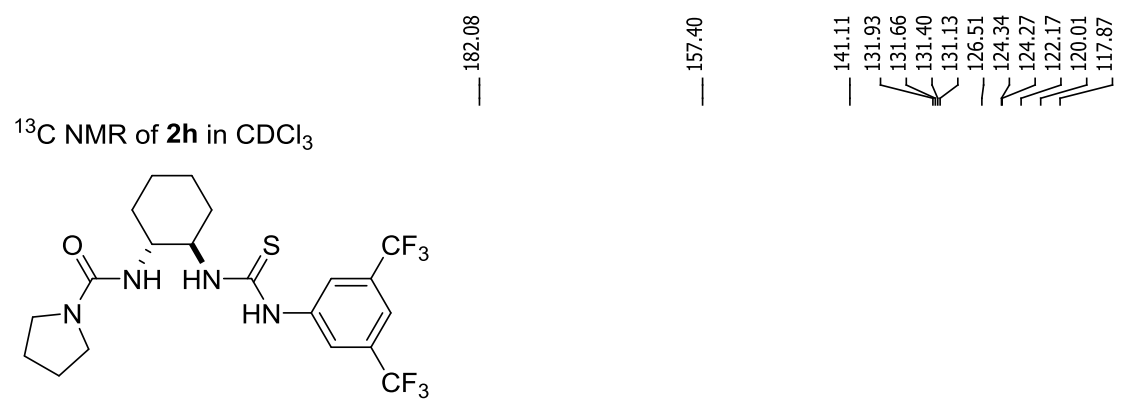

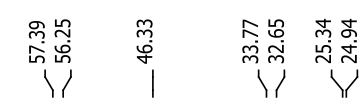

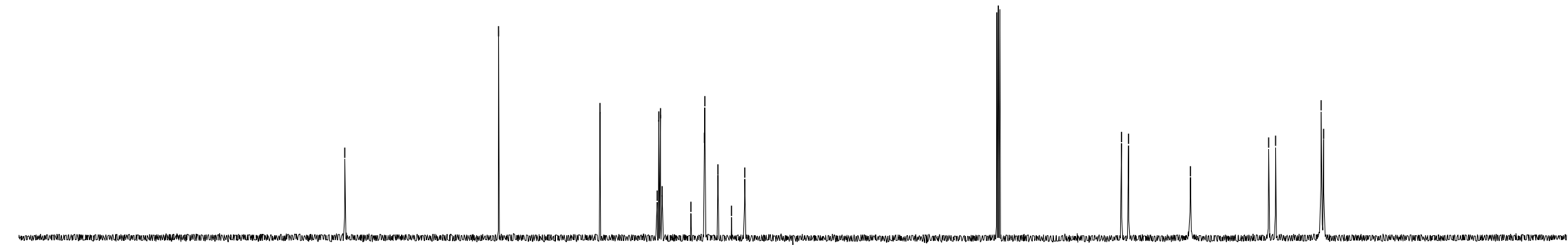

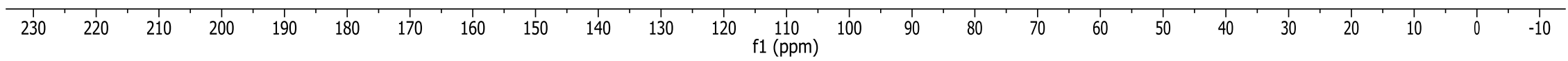




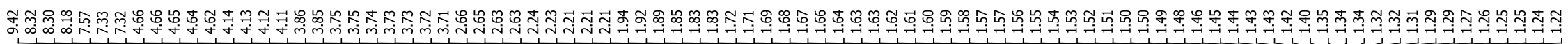
${ }^{1} \mathrm{H} \mathrm{NMR}$ of $\mathbf{2} \mathbf{i}$ in $\mathrm{CDCl}_{3}$<smiles>FC(F)(F)c1cc(NC(=S)NC2CCCCC2NC(=S)N2CCCCC2)cc(C(F)(F)F)c1</smiles>

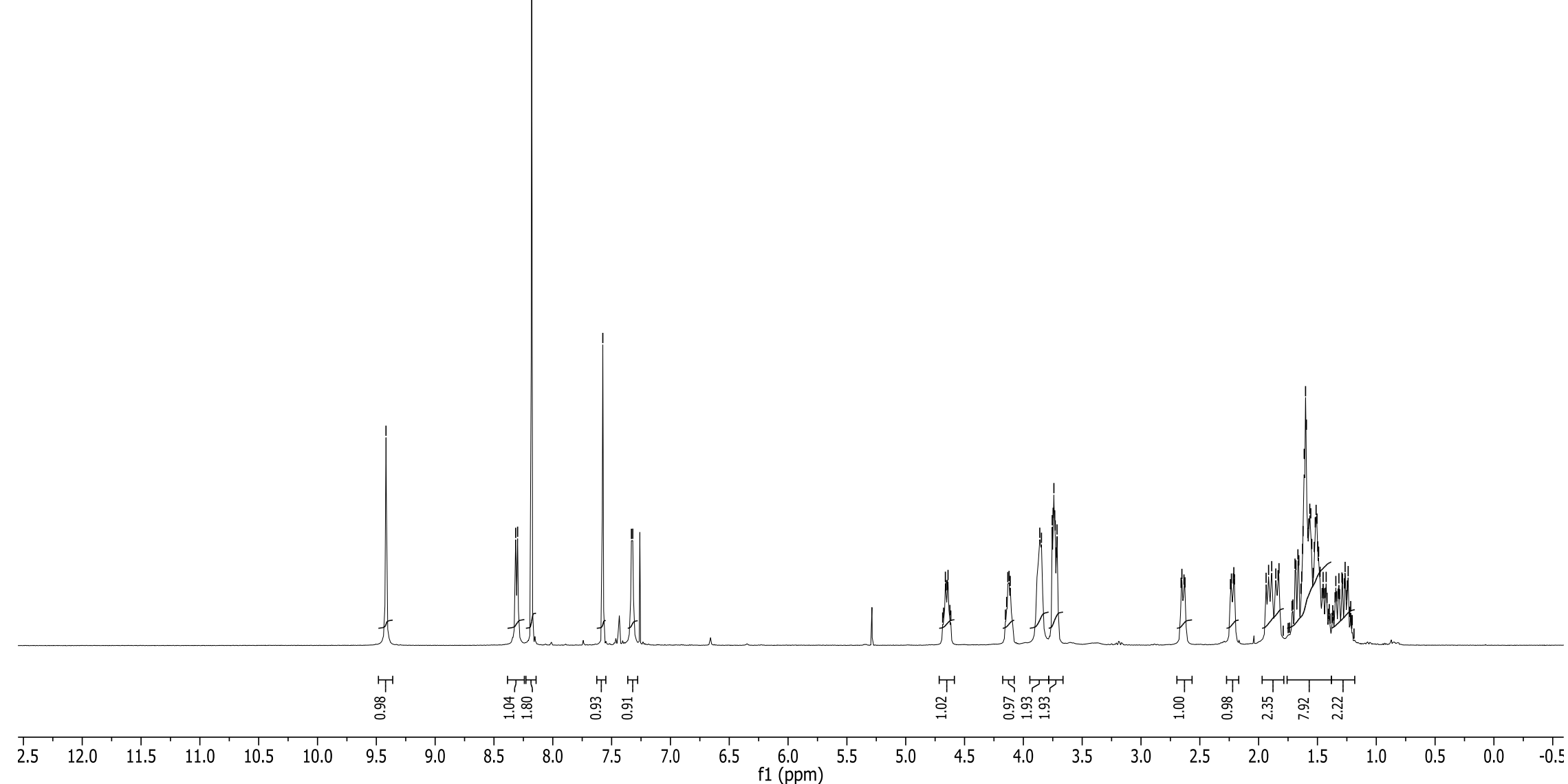




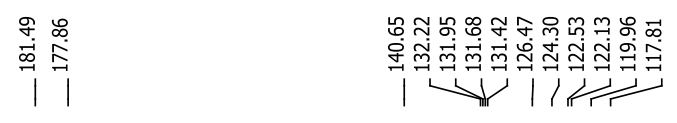

i̊

${ }^{13} \mathrm{C} \mathrm{NMR}$ of $\mathbf{2} \mathbf{i}$ in $\mathrm{CDCl}_{3}$
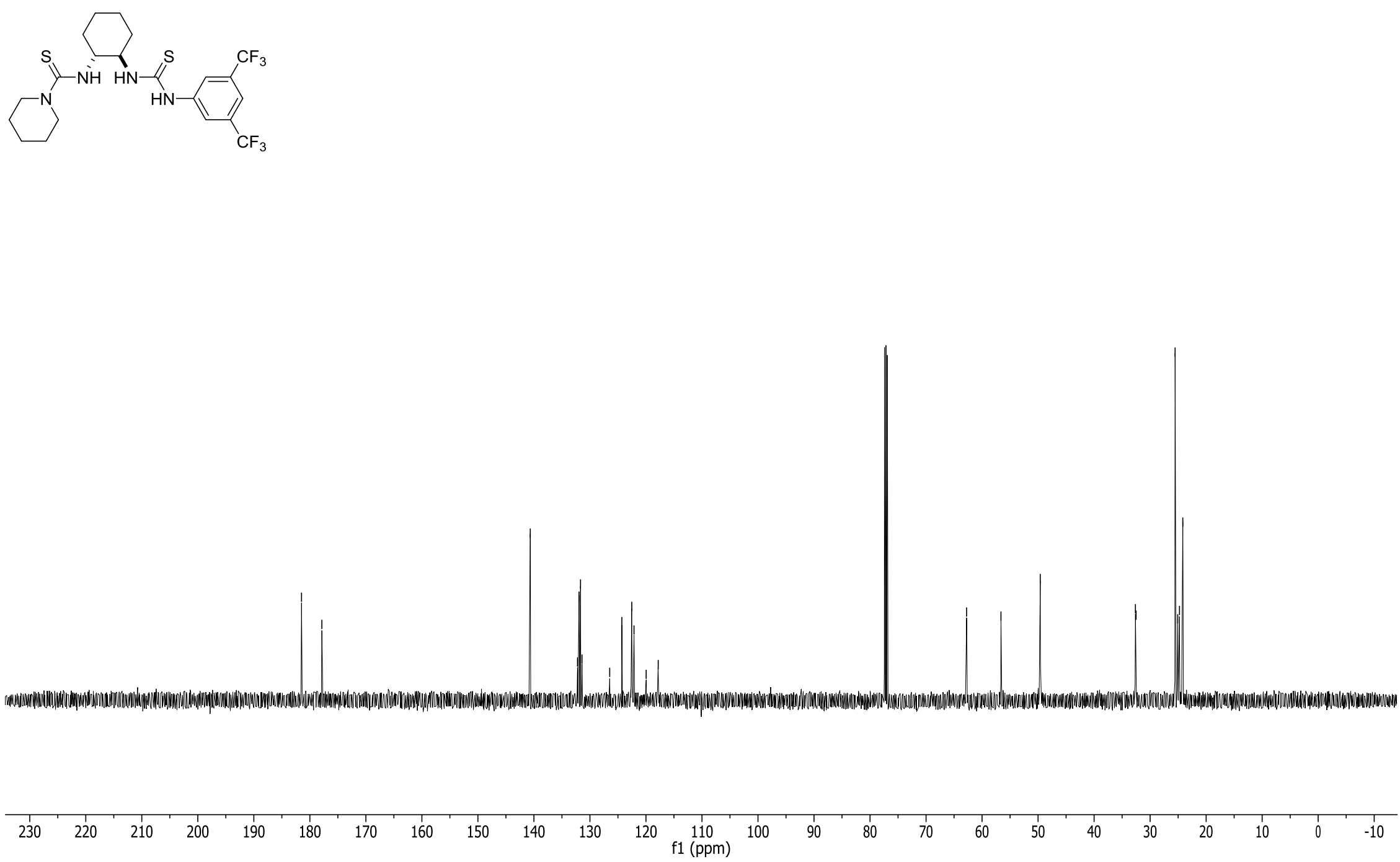
${ }^{1} \mathrm{H}$ NMR of $2 \mathbf{j}$ in $\mathrm{CDCl}_{3}$
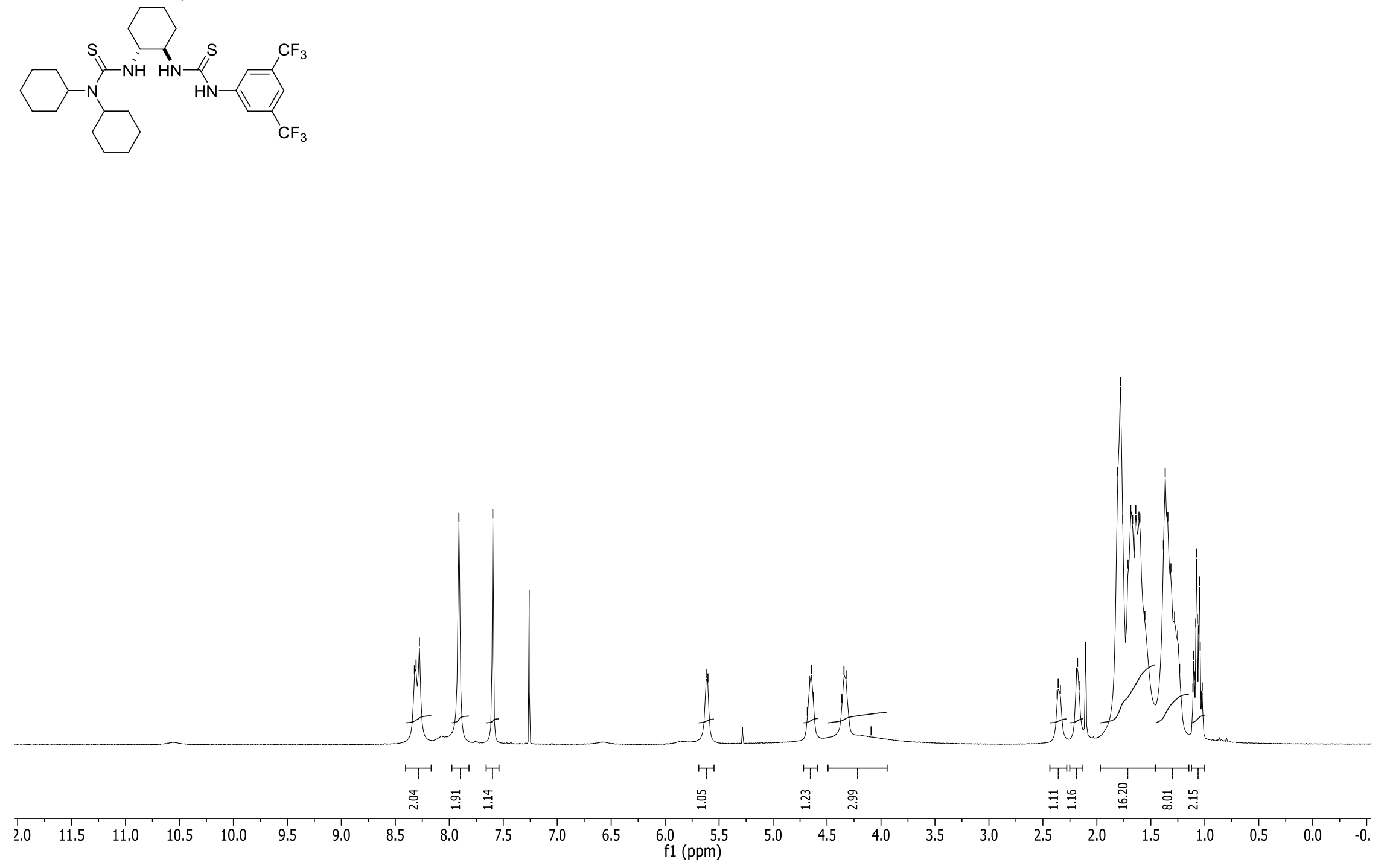

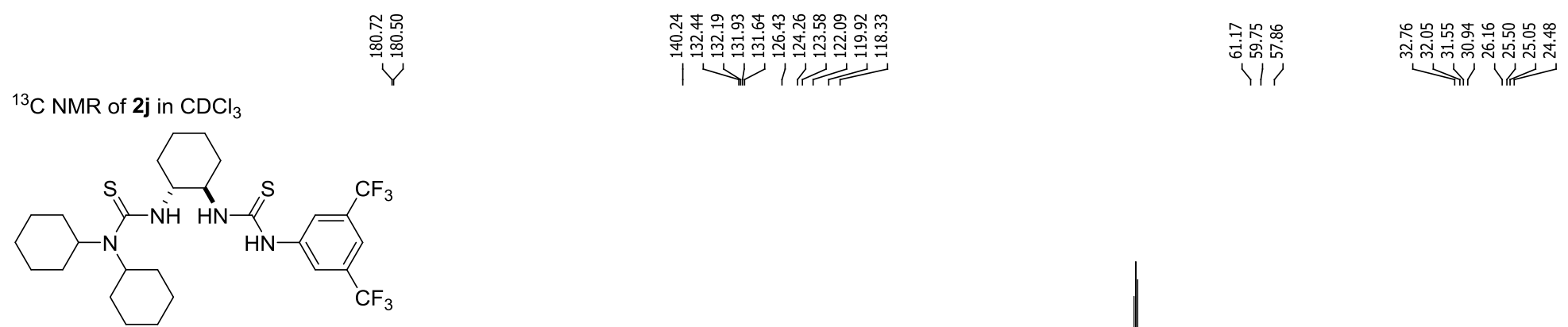


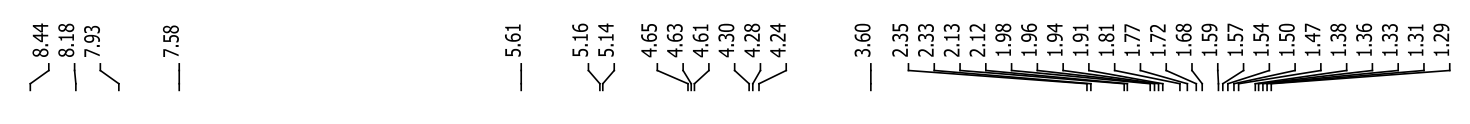

${ }^{1} \mathrm{H}$ NMR of $\mathbf{2 k}$ in $\mathrm{CDCl}_{3}$
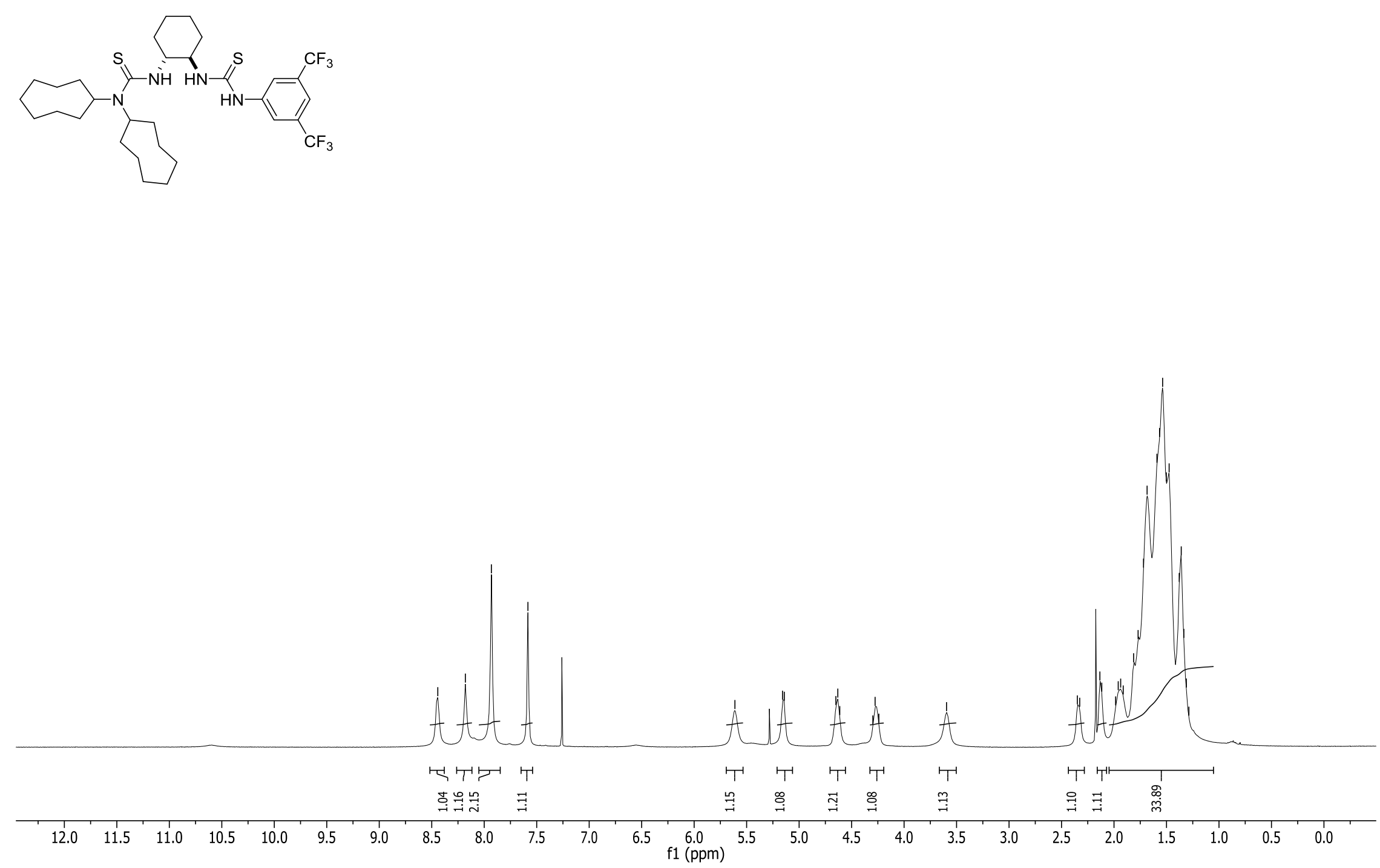


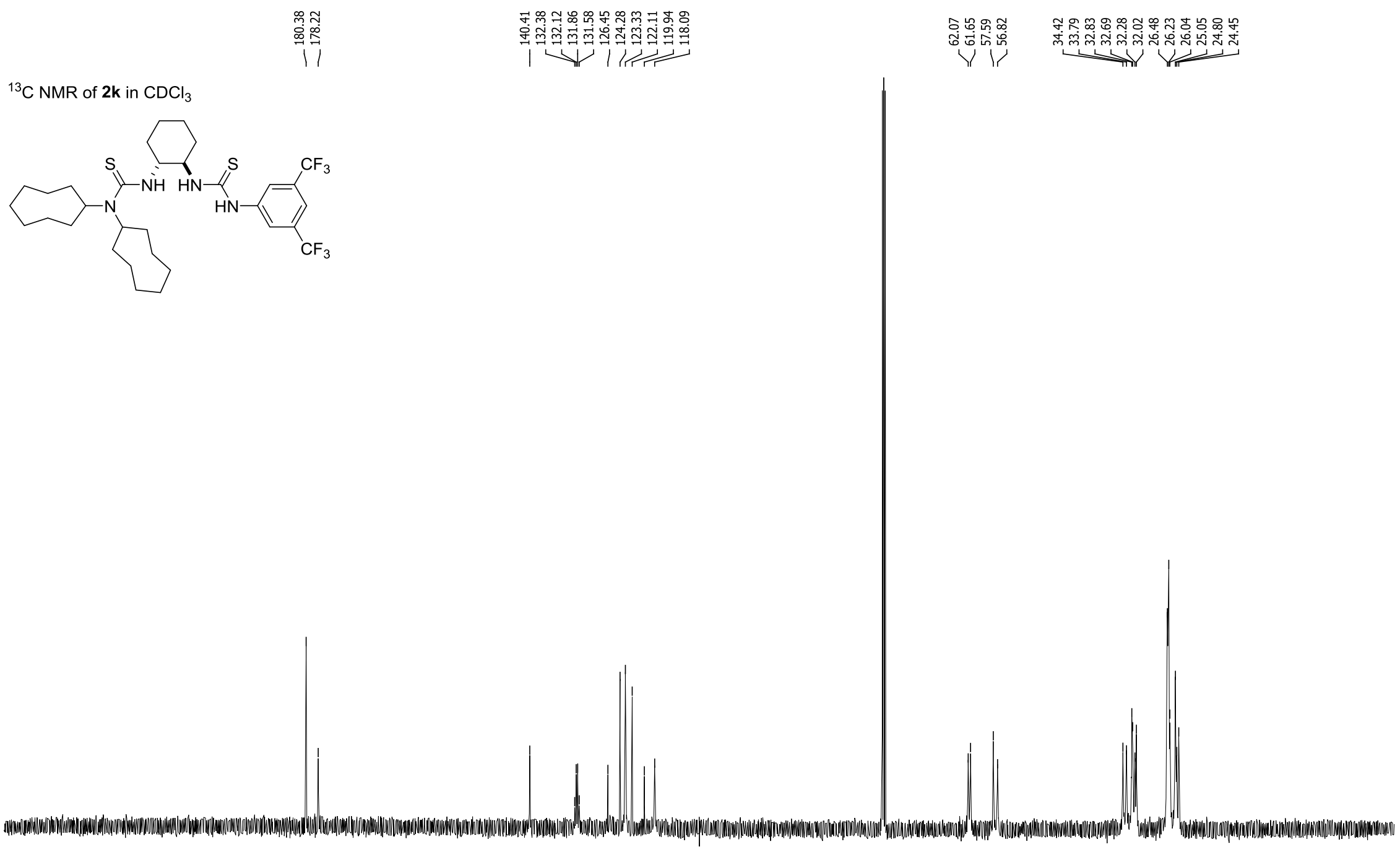

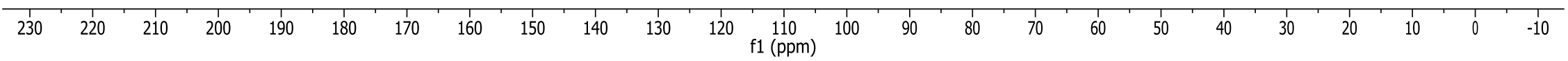


${ }^{1} \mathrm{H} \mathrm{NMR}$ in $\left(\mathrm{CD}_{3}\right)_{2} \mathrm{SO}$
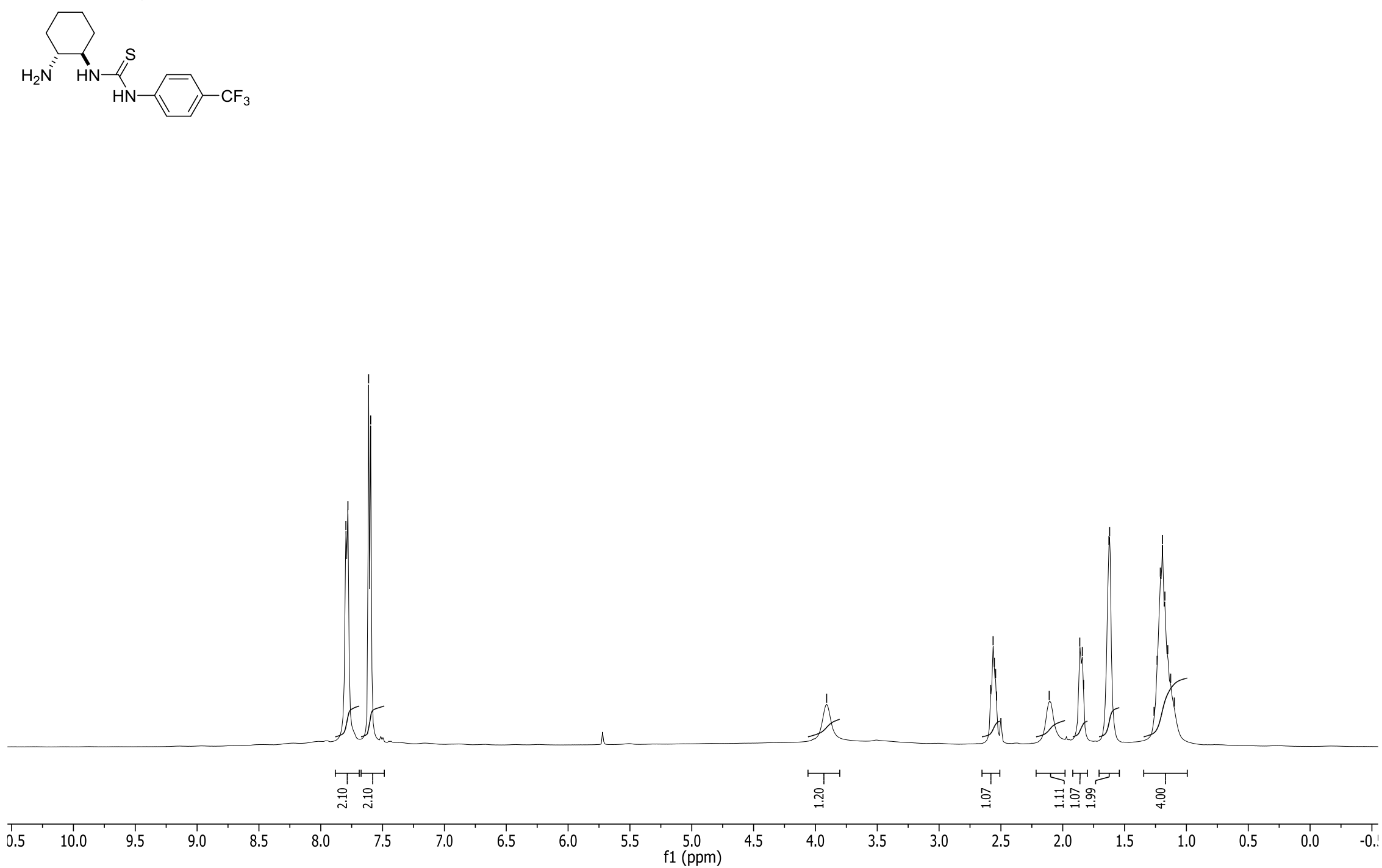
${ }^{13} \mathrm{C}$ NMR in $\left(\mathrm{CD}_{3}\right)_{2} \mathrm{SO}$
$\stackrel{\overrightarrow{0}}{\stackrel{\overrightarrow{0}}{\mid}}$
|

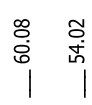

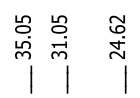

$\mathrm{H}_{2} \mathrm{~N}$

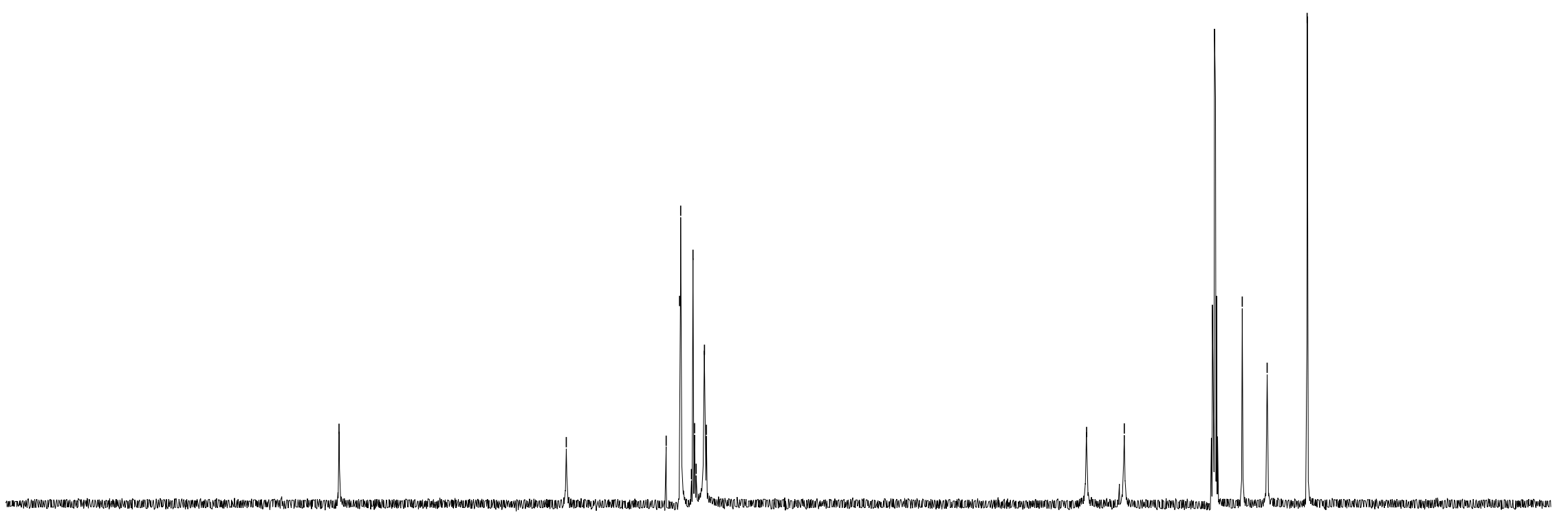

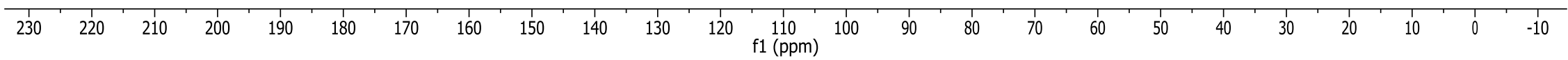




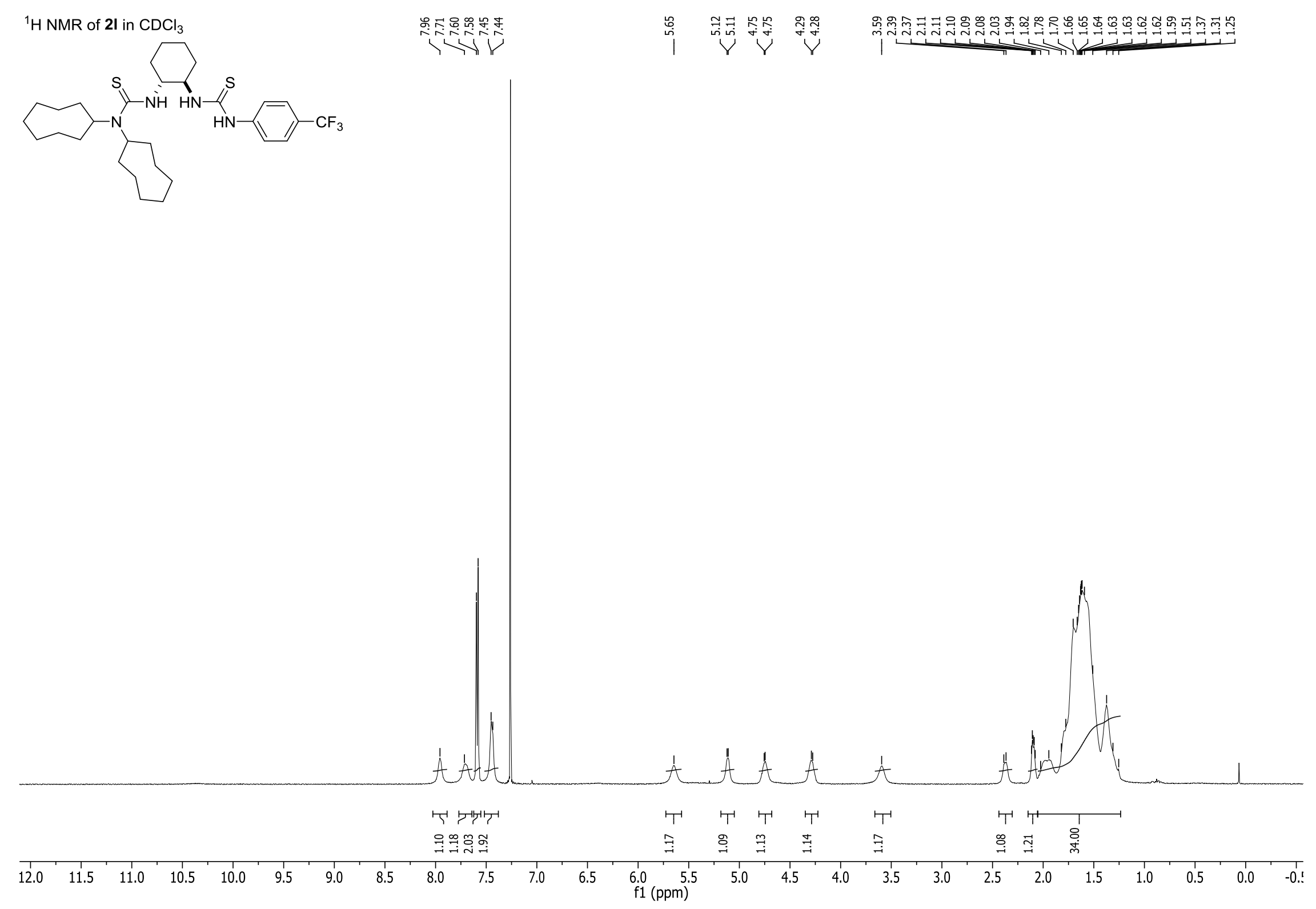



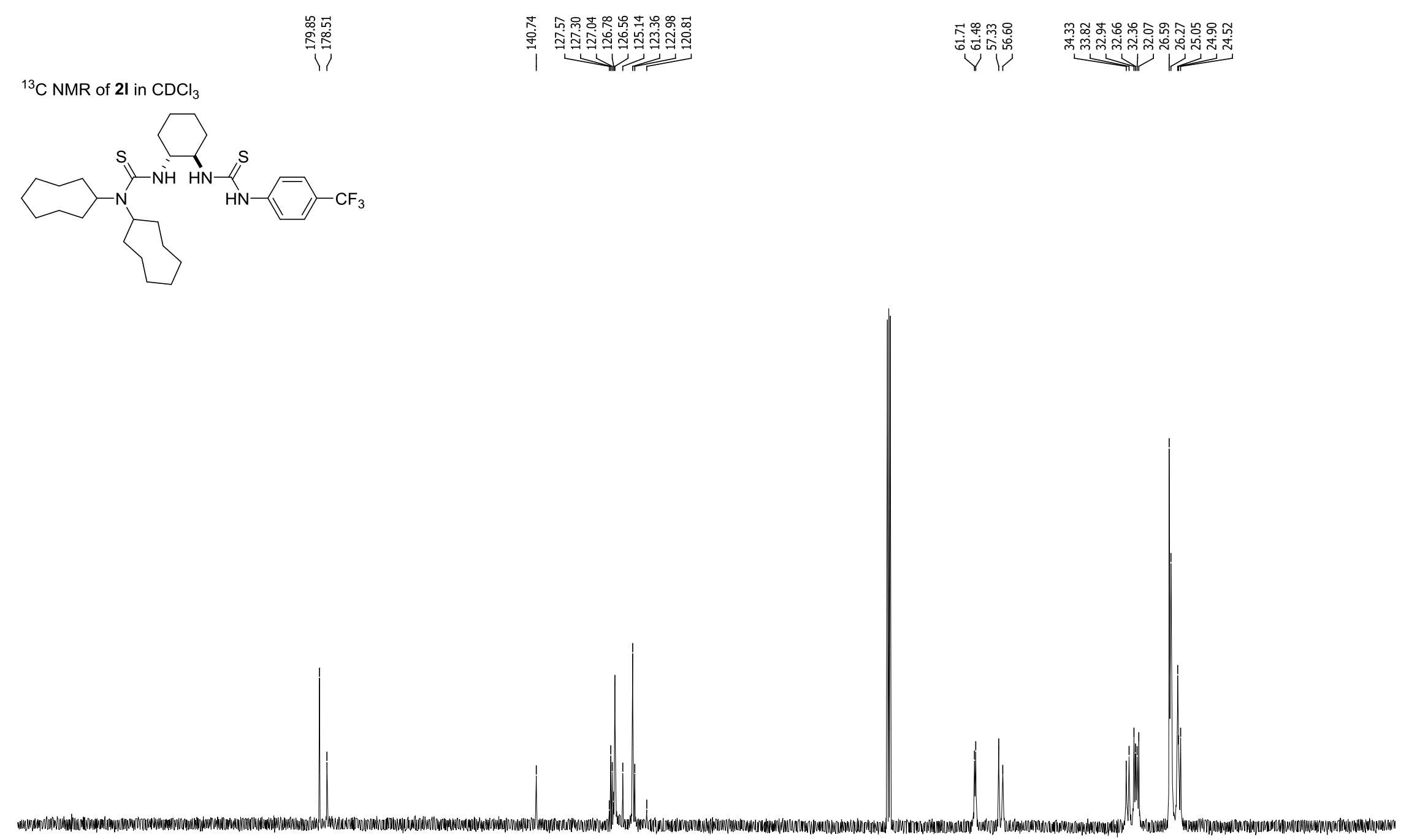

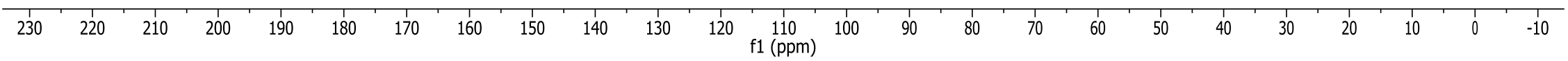




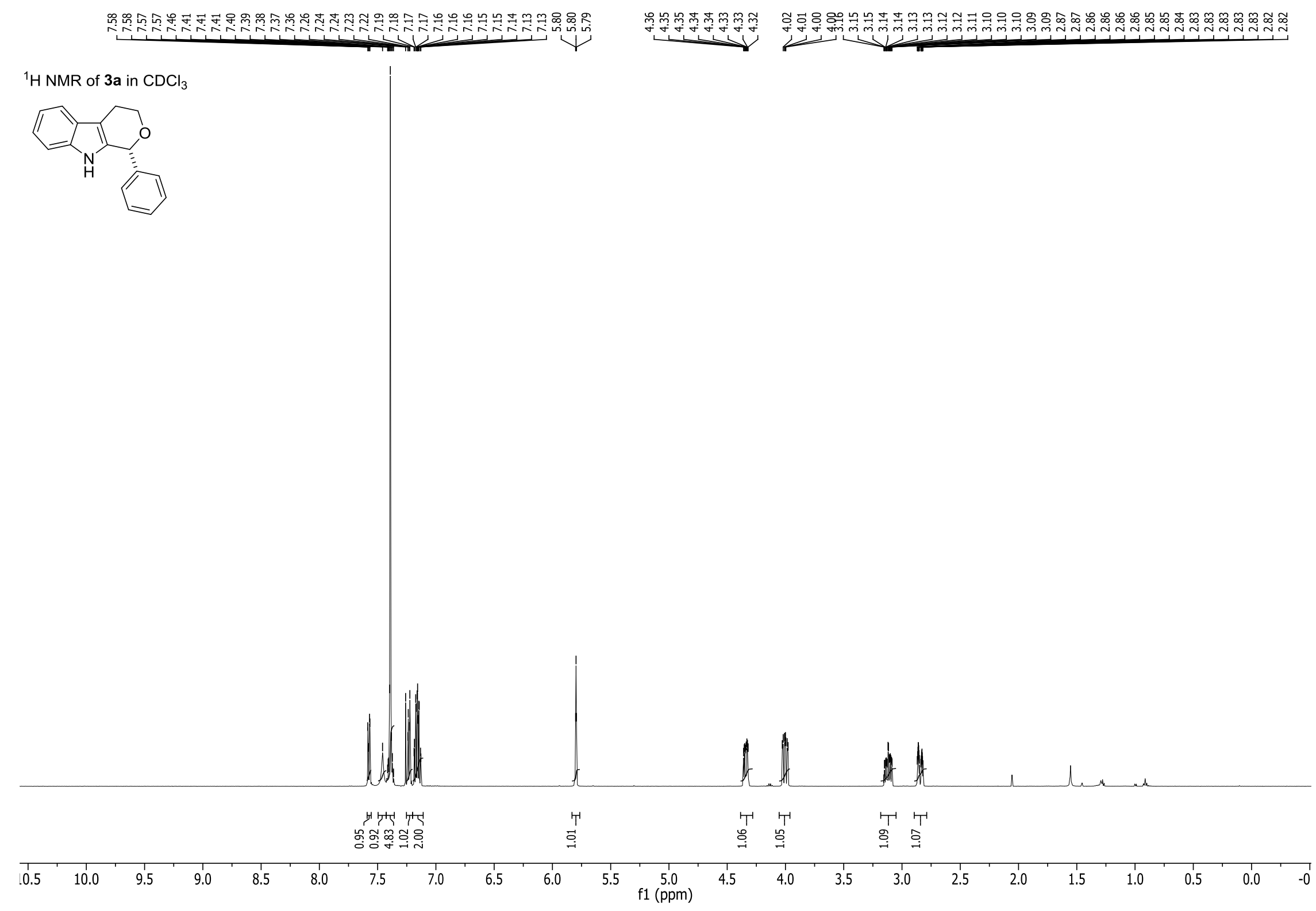




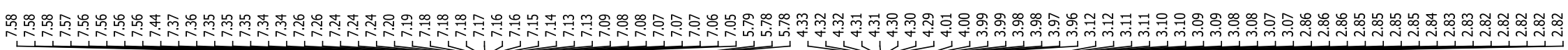
${ }^{1} \mathrm{H} N M R$ of $3 b$ in $\mathrm{CDC}$

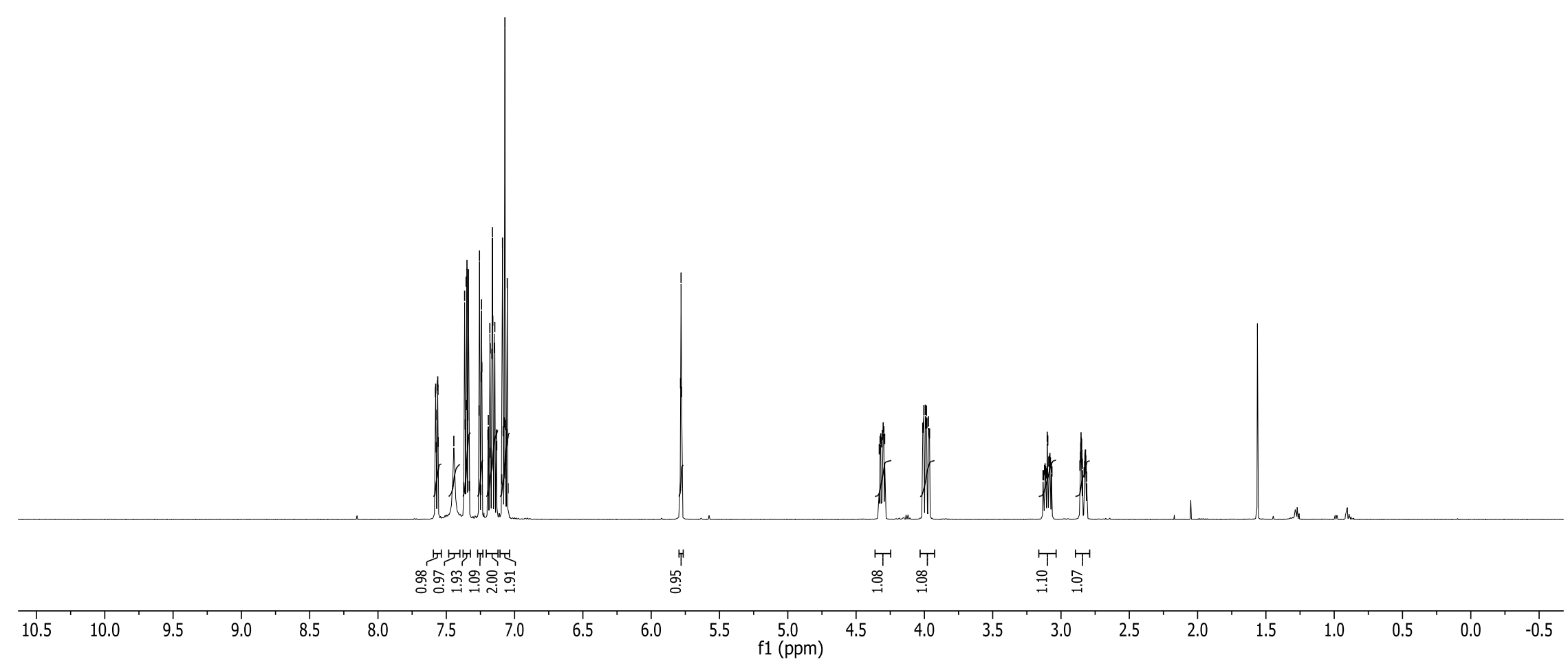


${ }^{13} \mathrm{C} \mathrm{NMR}$ of $\mathbf{3 b}$ in $\mathrm{CDCl}_{3}$

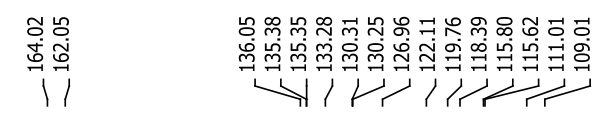

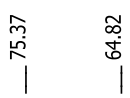

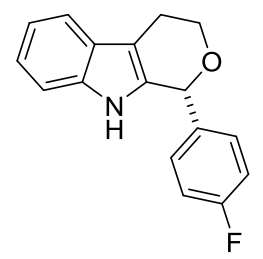

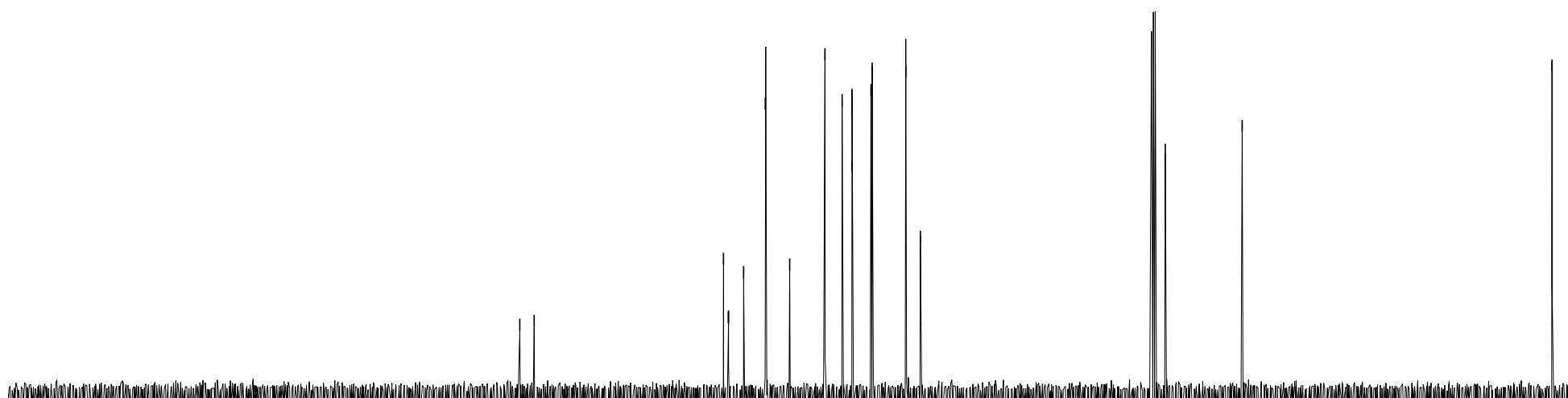

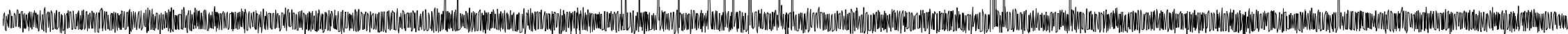

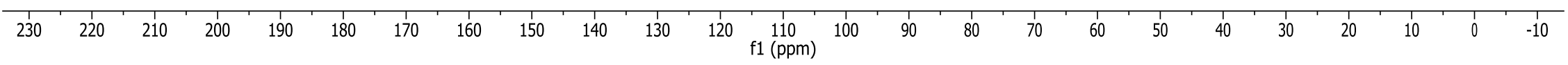


${ }^{1} \mathrm{H} \mathrm{NMR}$ of $3 \mathrm{c}$ in $\mathrm{CDCl}_{3}$
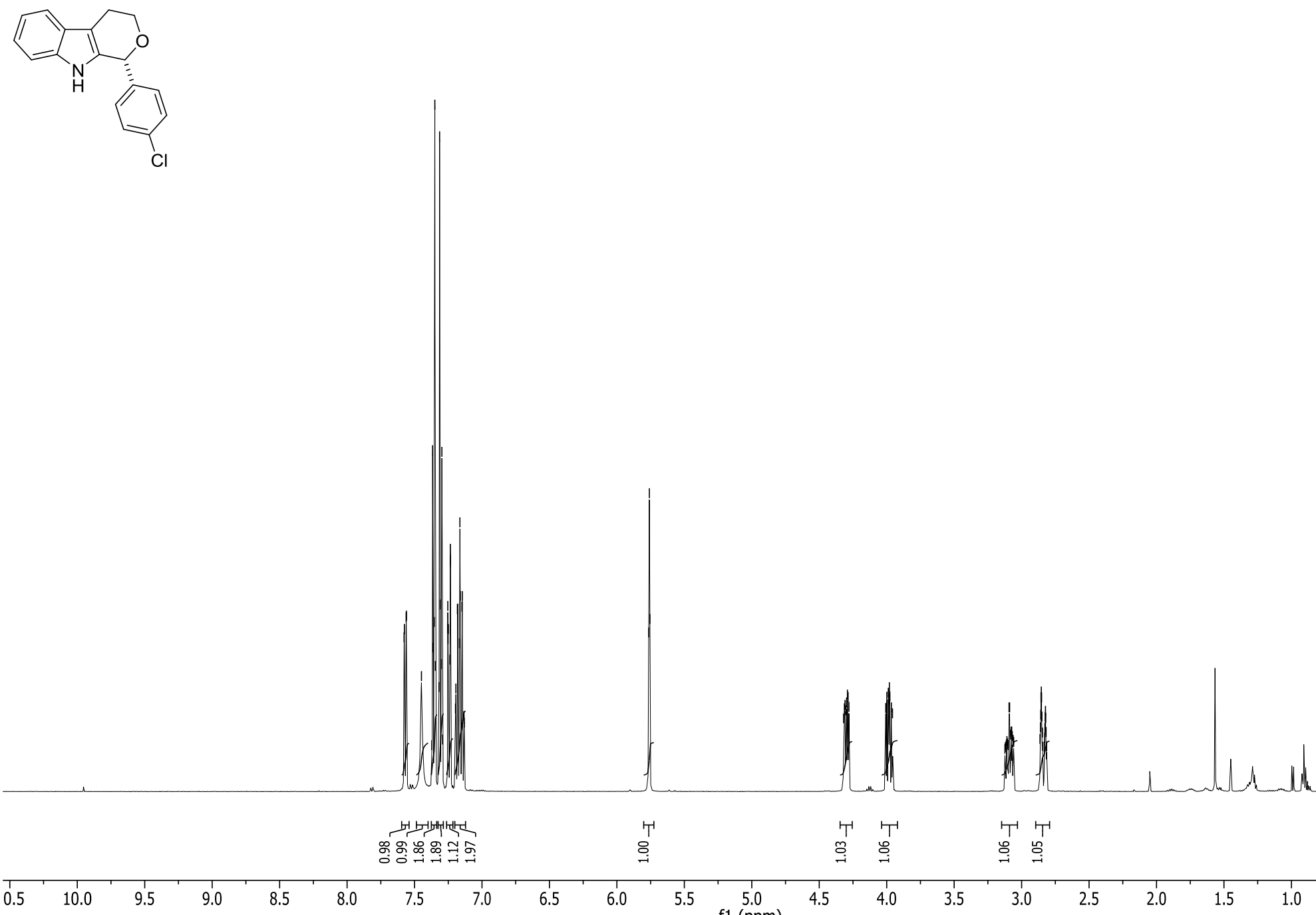

9.0

8.5

8.0

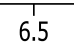

6.0

$\begin{array}{cc}5.5 & 5.0 \\ f 1(\mathrm{ppm})\end{array}$ 


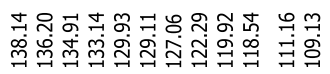

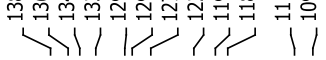

${ }^{13} \mathrm{C} \mathrm{NMR}$ of $3 \mathrm{c}$ in $\mathrm{CDCl}_{3}$

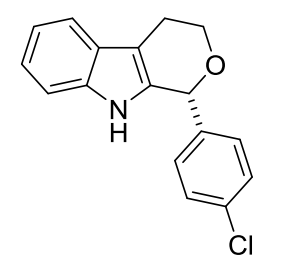

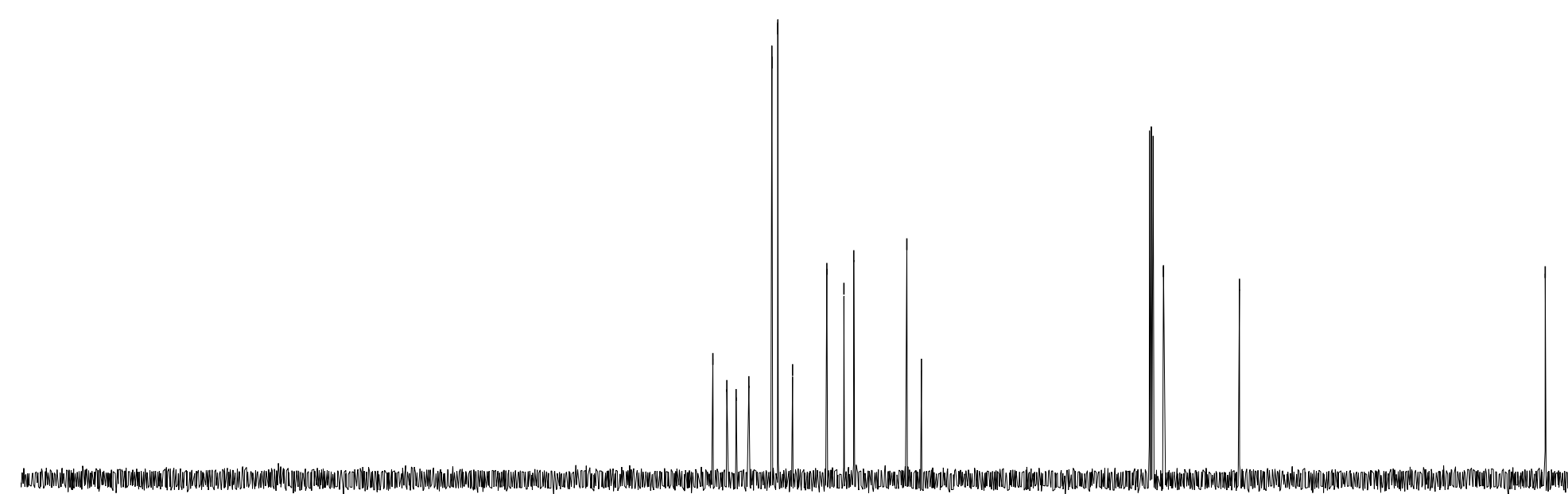

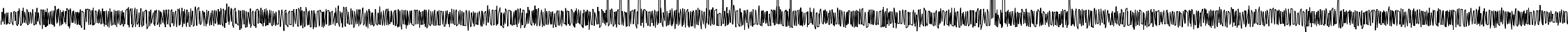

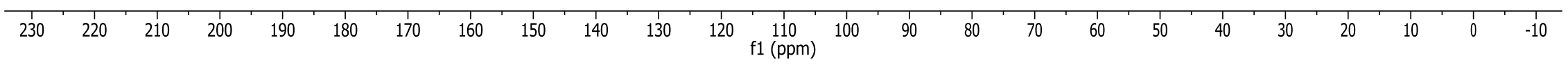




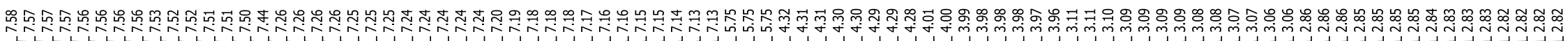
${ }^{1} \mathrm{H} \mathrm{NMR}$ of $3 \mathbf{d}$ in $\mathrm{CDCl}_{3}$

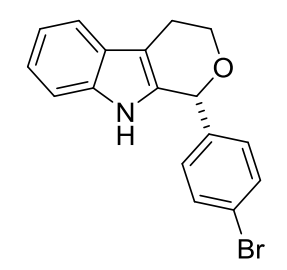

$\mathrm{Br}$

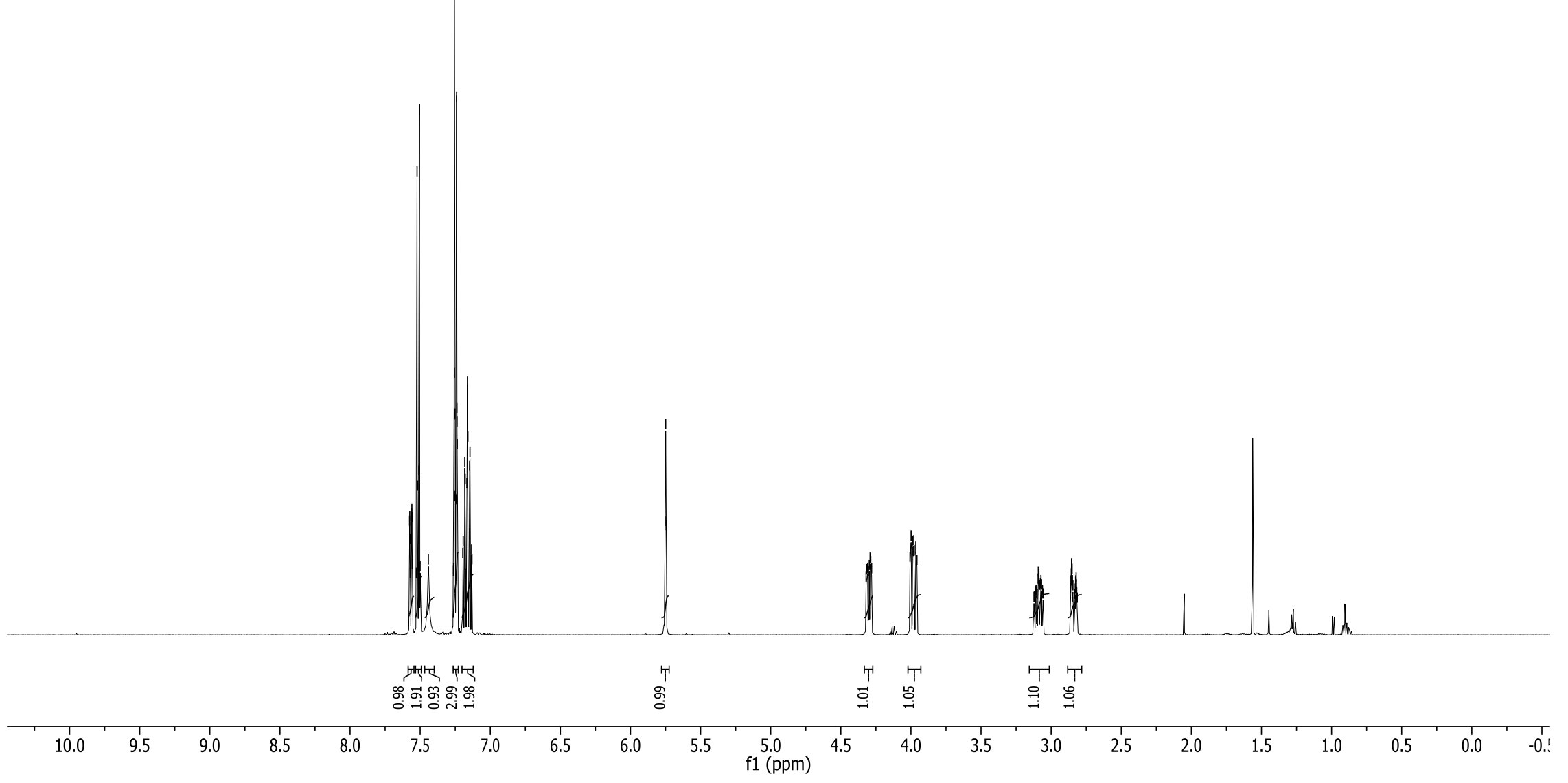


${ }^{13} \mathrm{C} \mathrm{NMR}$ of $\mathbf{3 d}$ in $\mathrm{CDCl}_{3}$

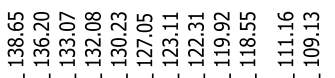<smiles>Brc1ccc([C@@H]2OCCc3c2[nH]c2ccccc32)cc1</smiles>

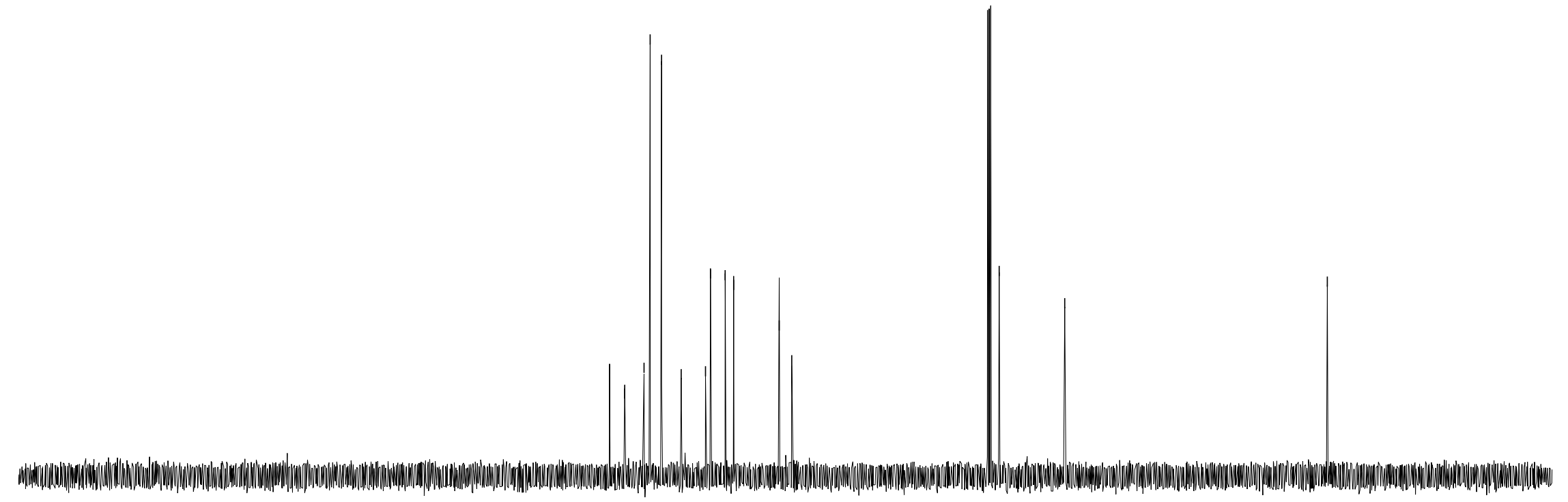

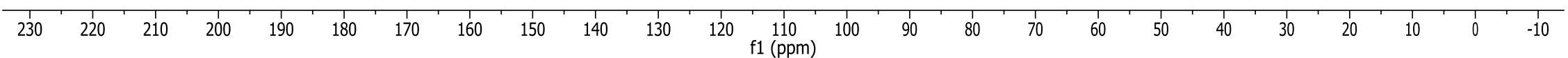




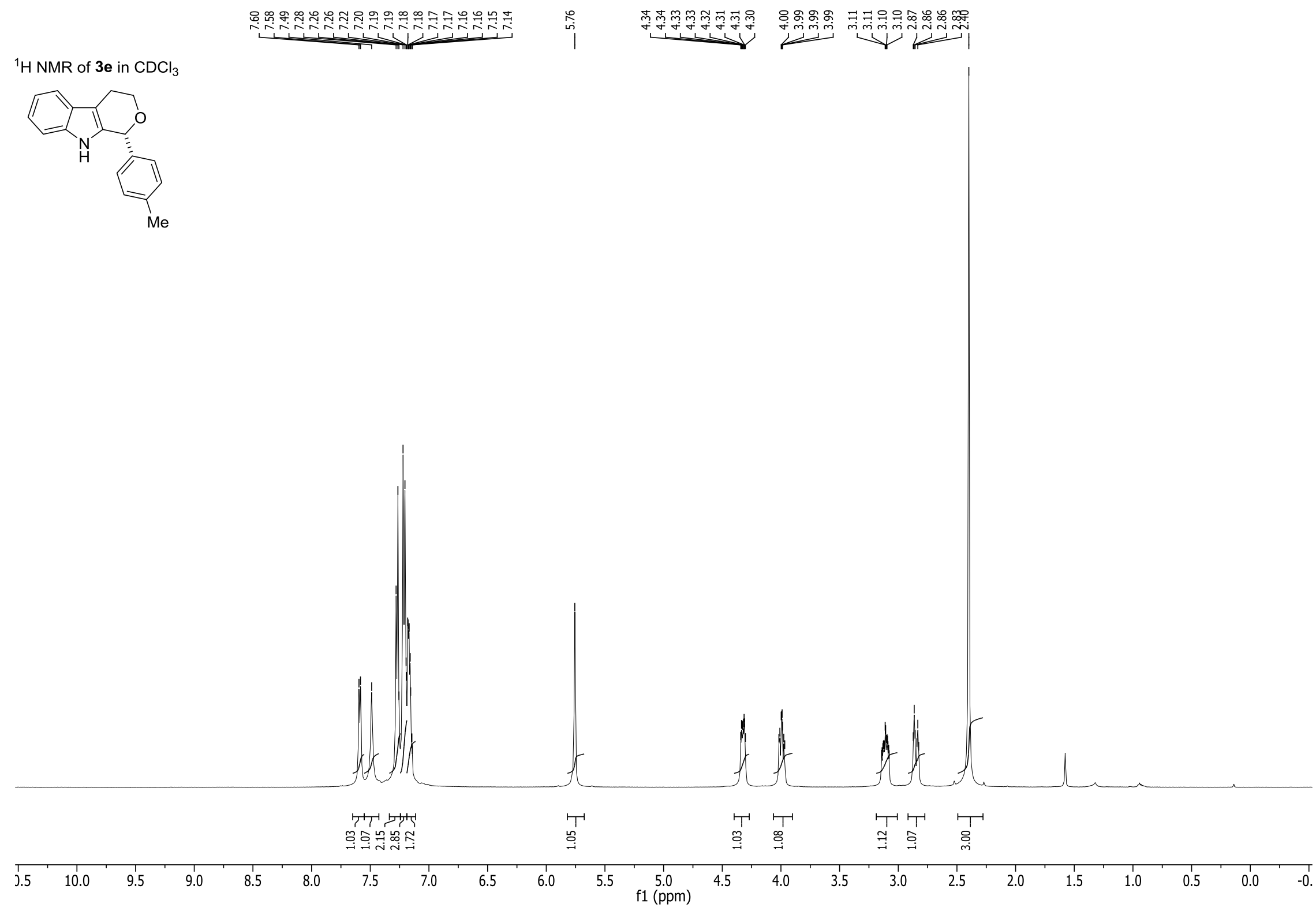


${ }^{13} \mathrm{C} \mathrm{NMR}$ of $3 \mathbf{e}$ in $\mathrm{CDCl}_{3}$
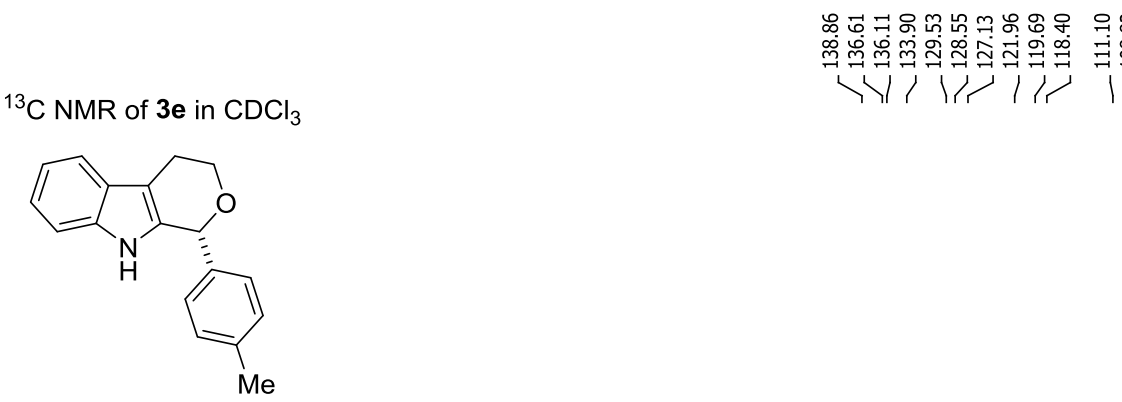

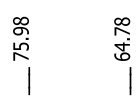

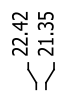

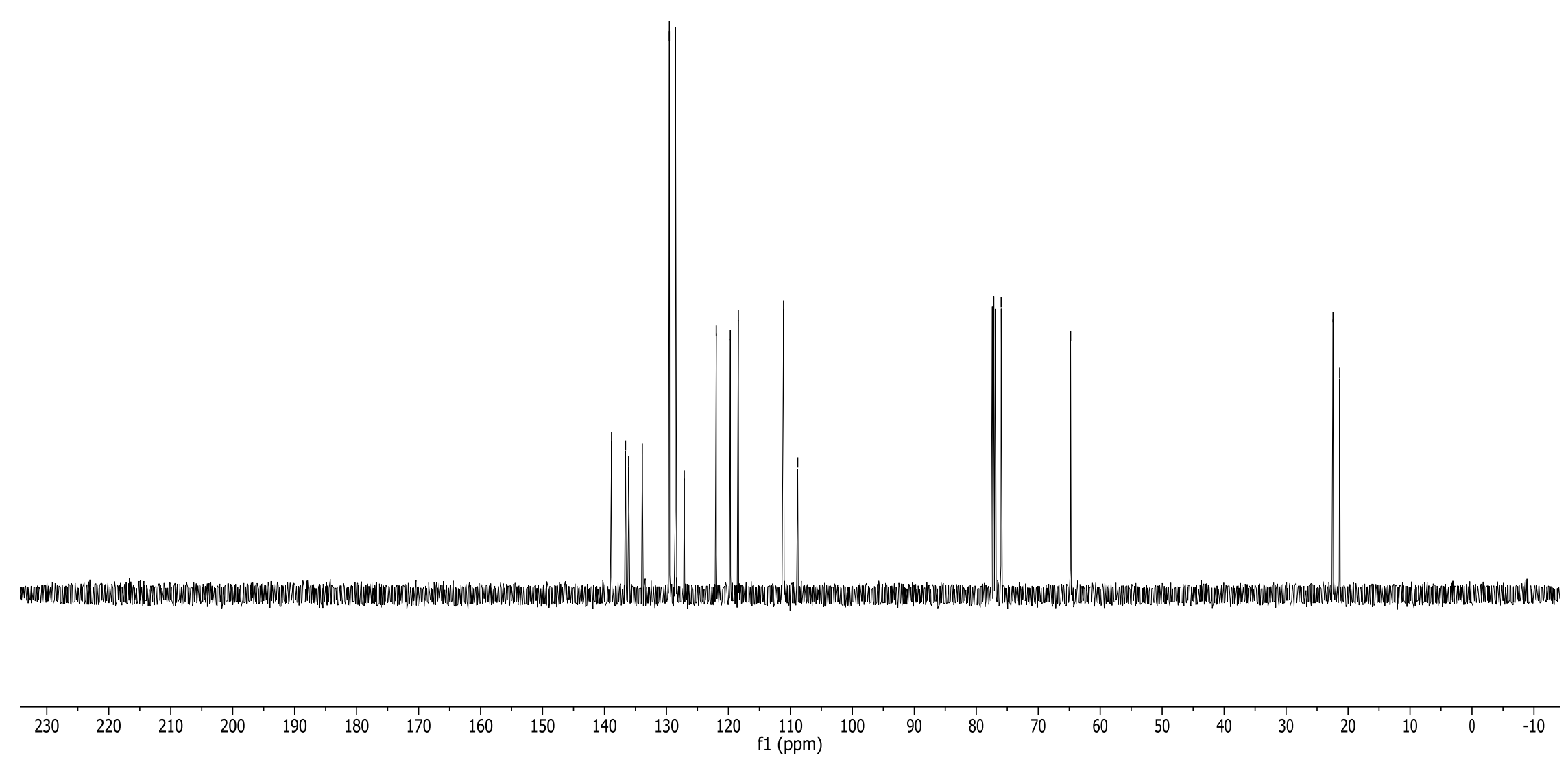




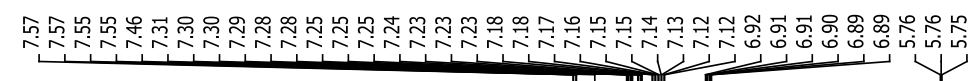

${ }^{1} \mathrm{H} \mathrm{NMR}$ of $\mathbf{3 f}$ in $\mathrm{CDCl}_{3}$

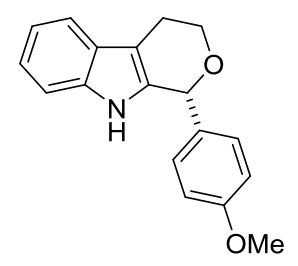

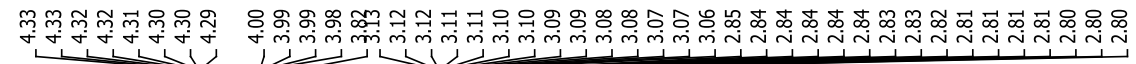

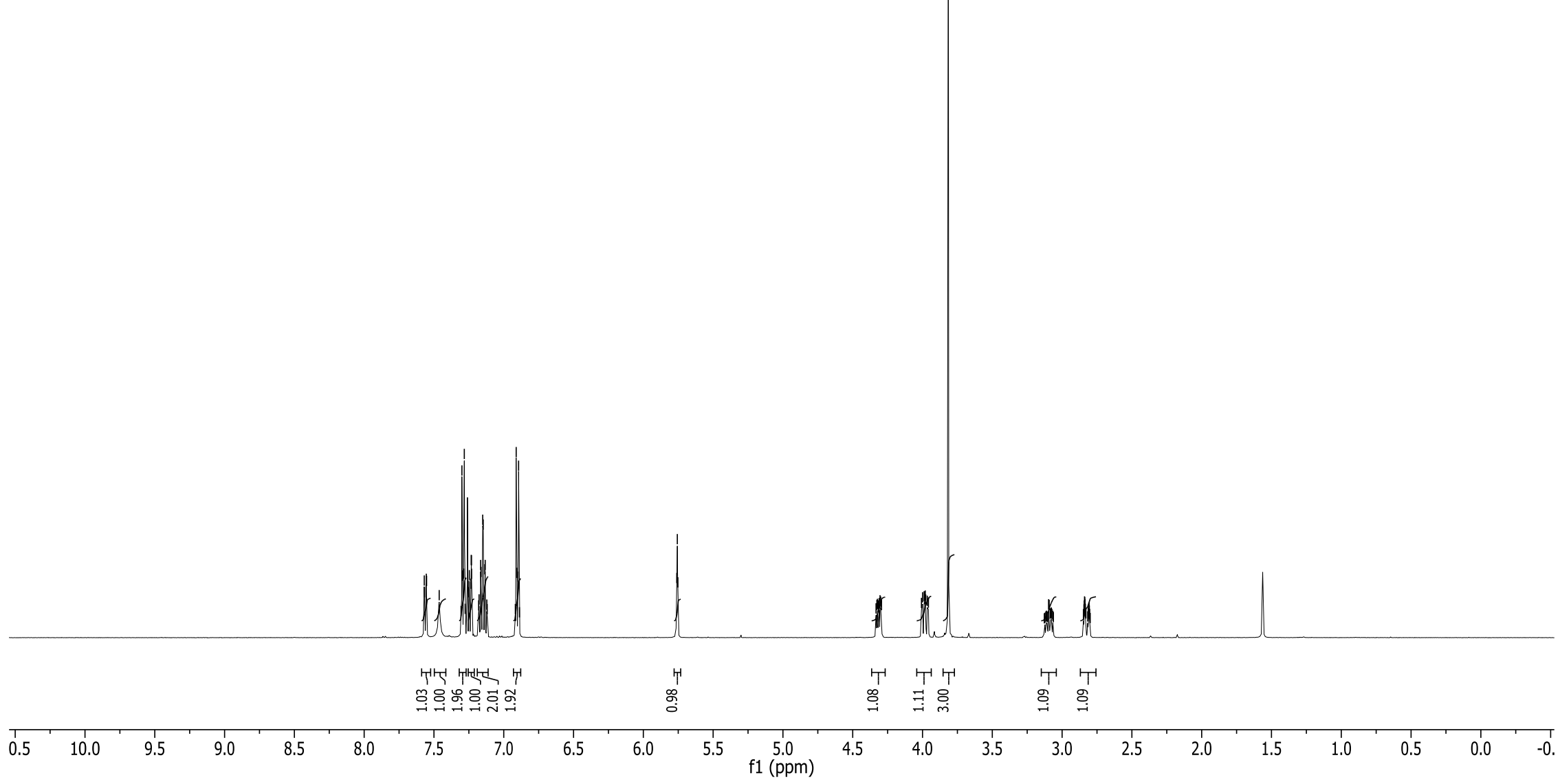


${ }^{13} \mathrm{C} \mathrm{NMR}$ of $3 \mathbf{f}$ in $\mathrm{CDCl}_{3}$

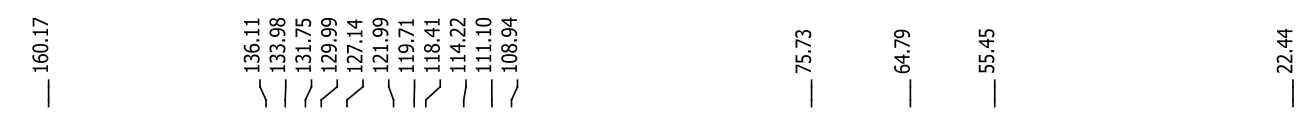

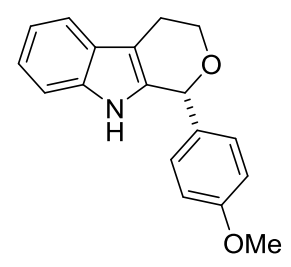

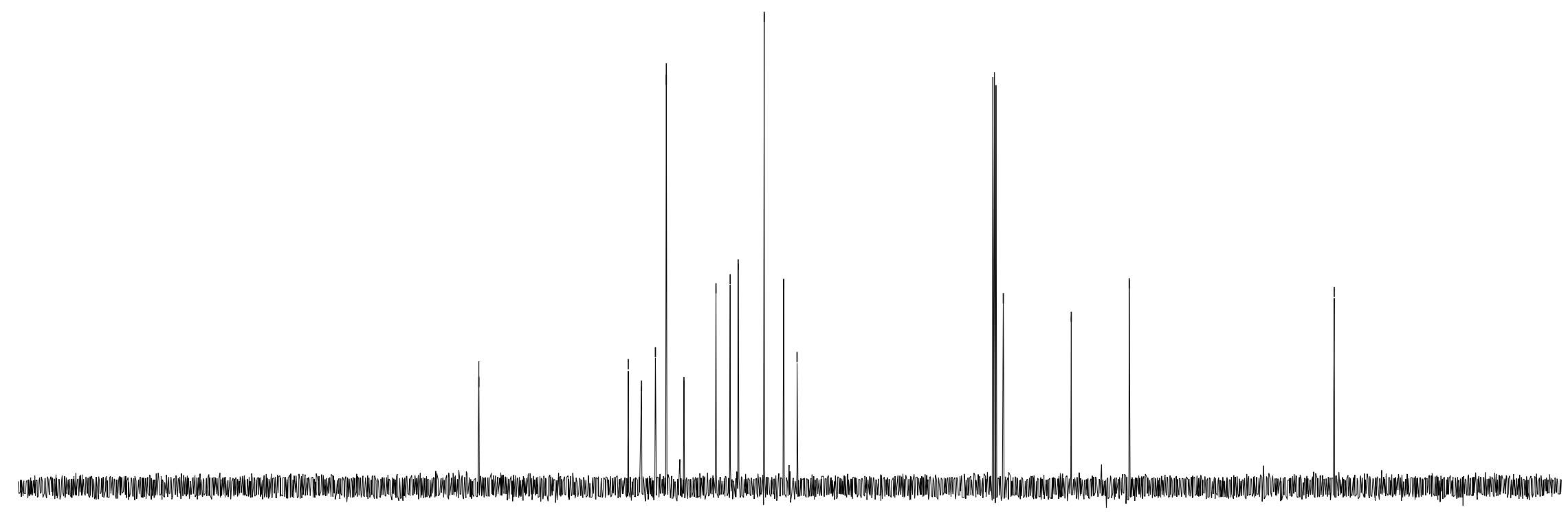

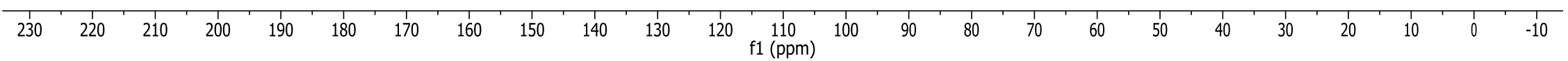




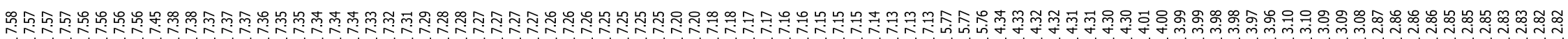
(1) ${ }^{1} \mathrm{H} \mathrm{NMR}$ of $\mathbf{3 g}$ in $\mathrm{CDCl}_{3}$
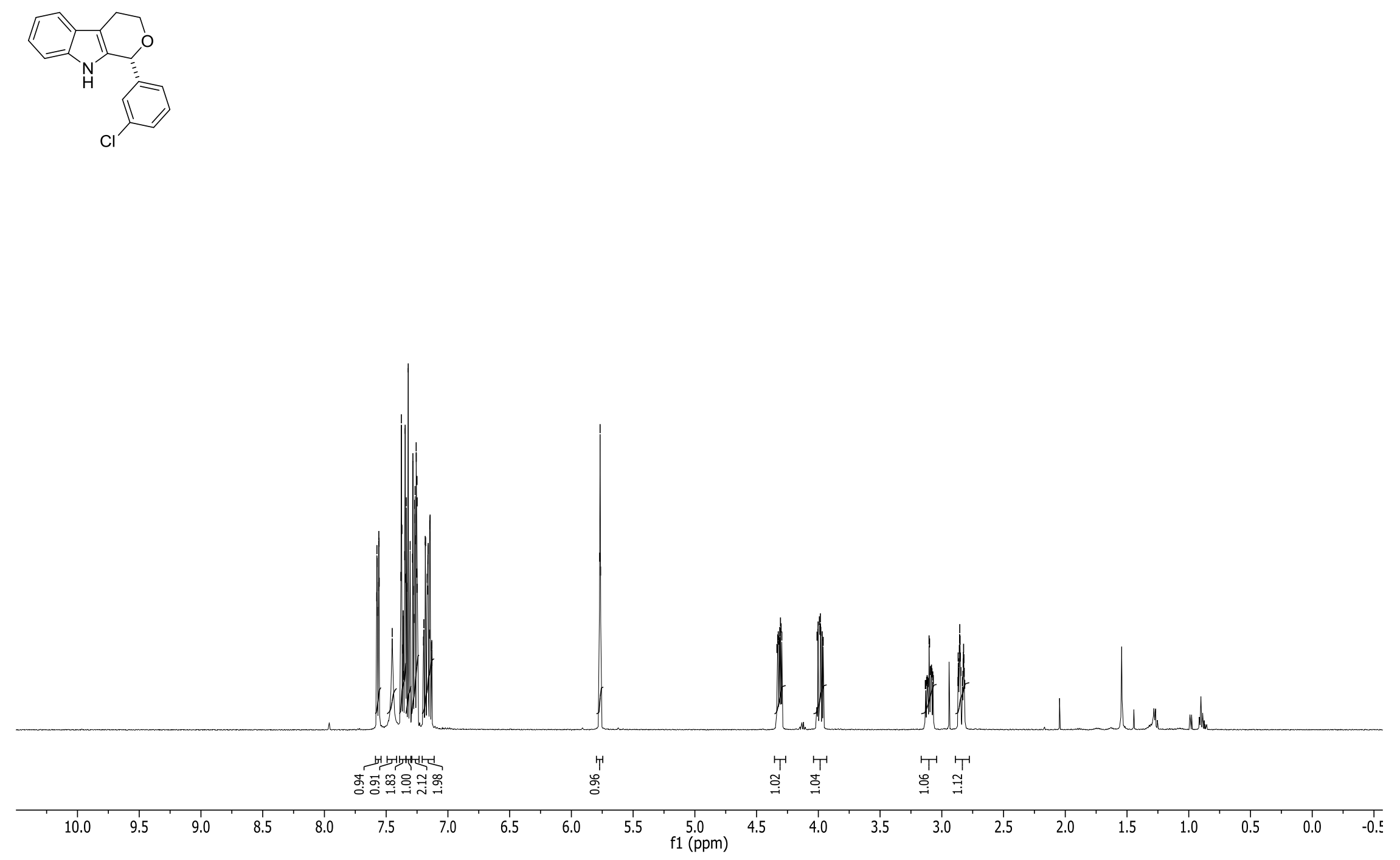
${ }^{13} \mathrm{C} \mathrm{NMR}$ of $\mathbf{3 g}$ in $\mathrm{CDCl}_{3}$

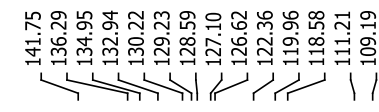

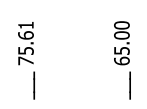

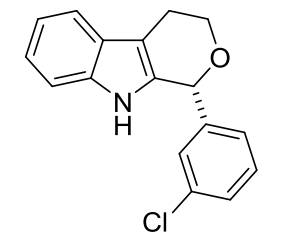

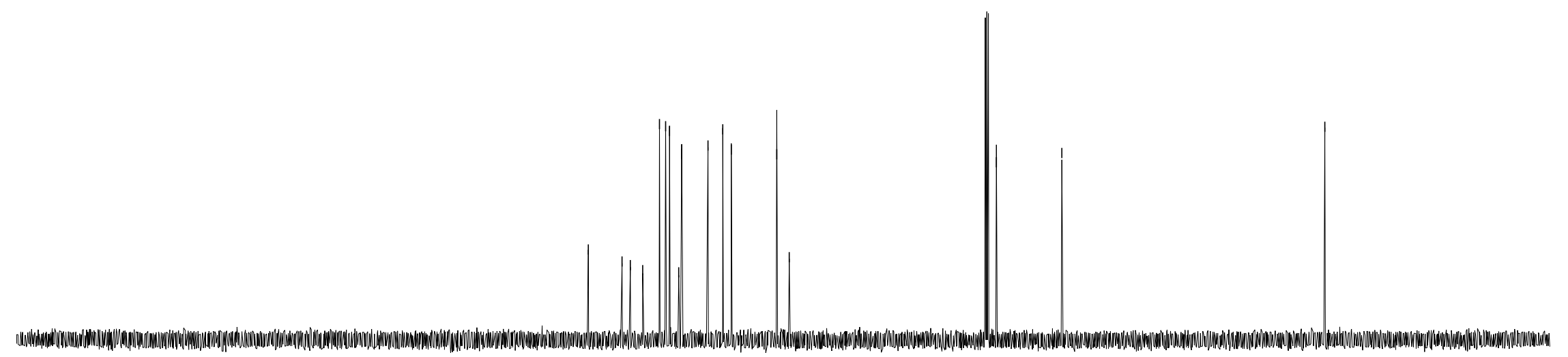

\begin{tabular}{llllllllllllllllllllllllllllll}
\hline 1 & 1 \\
230 & 220 & 210 & 200 & 190 & 180 & 170 & 160 & 150 & 140 & 130 & 120 & 110 & 100 & 90 & 80 & 70 & 60 & 50 & 40 & 30 & 20 & 10 & 0 & -10
\end{tabular}




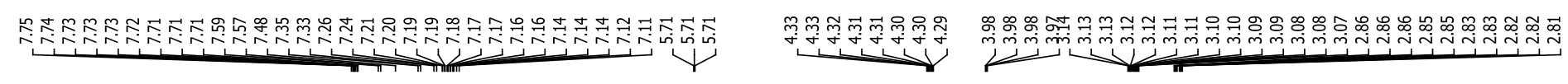
${ }^{1} \mathrm{H}$ NMR of $3 \mathbf{h}$ in $\mathrm{CDCl}_{3}$
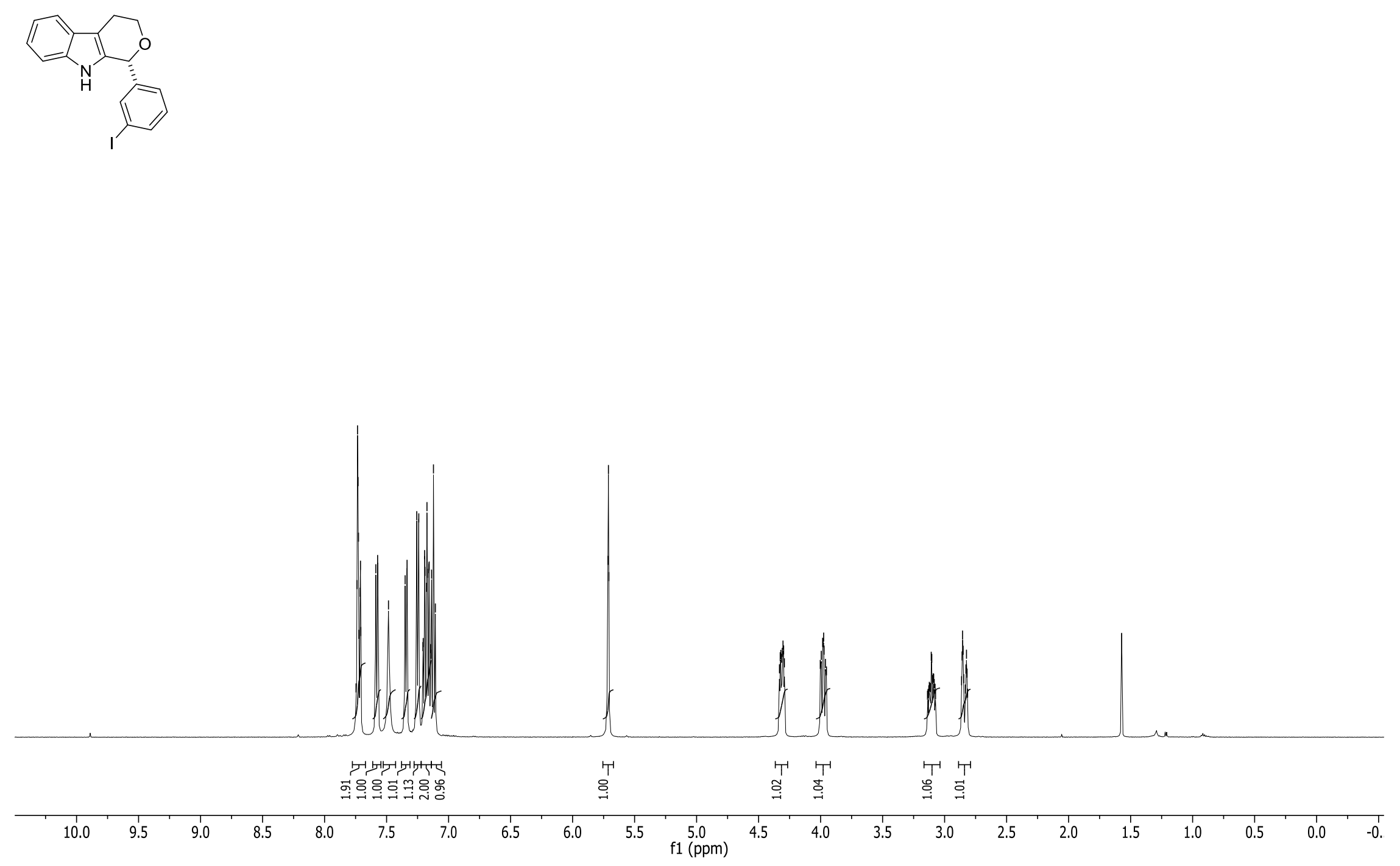
${ }^{13} \mathrm{C} \mathrm{NMR}$ of $\mathbf{3 h}$ in $\mathrm{CDCl}_{3}$

|

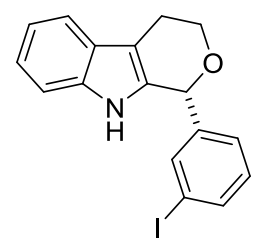

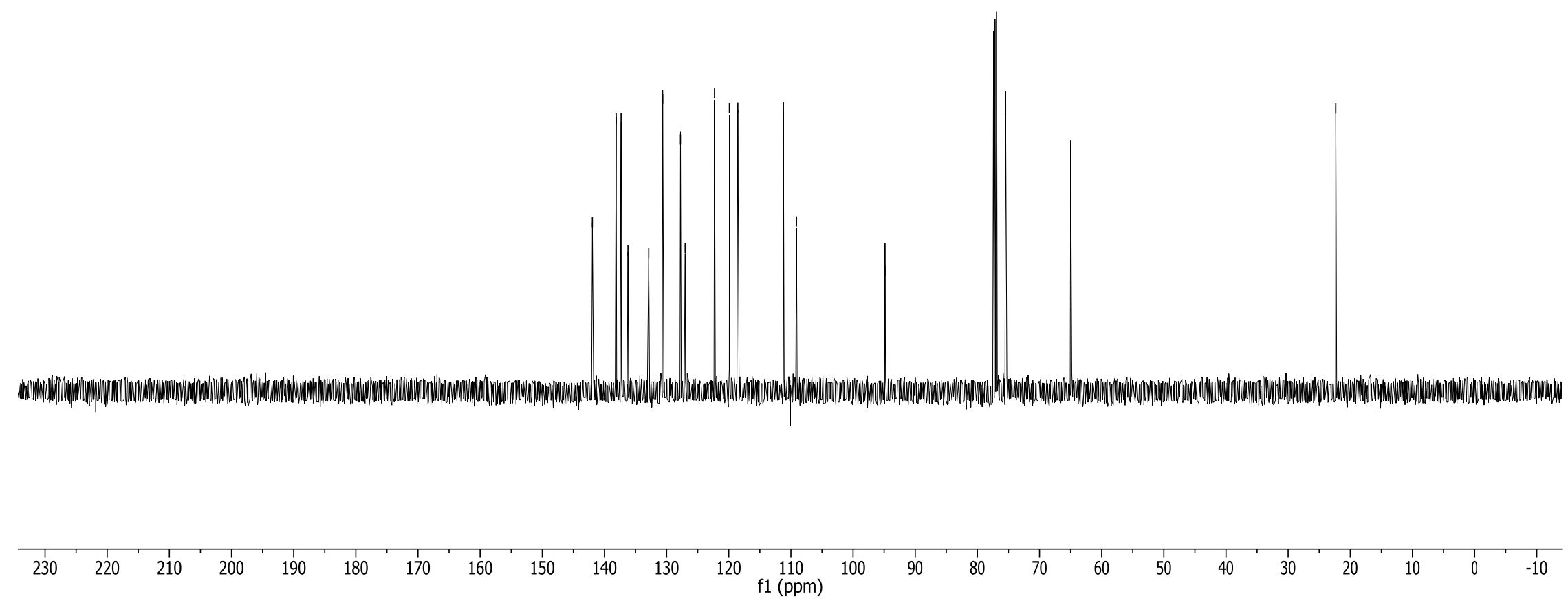




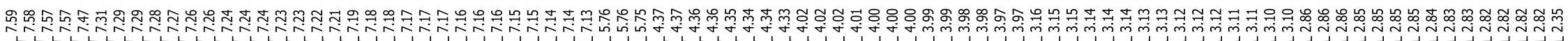
${ }^{1} \mathrm{H} \mathrm{NMR}$ of $3 \mathrm{i}$ in $\mathrm{CDCl}_{3}$
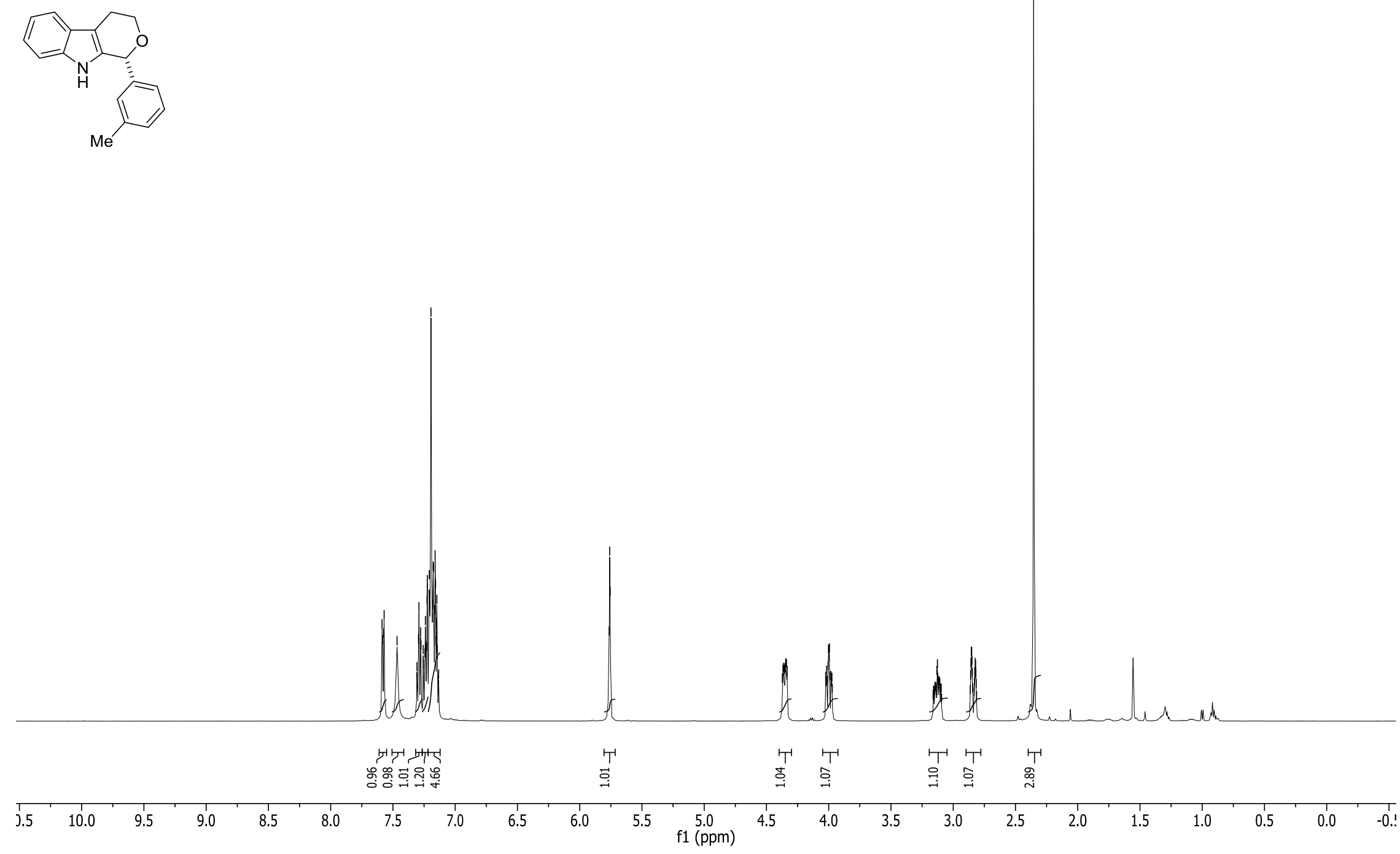
${ }^{13} \mathrm{C} \mathrm{NMR}$ of $3 \mathbf{i}$ in $\mathrm{CDCl}_{3}$

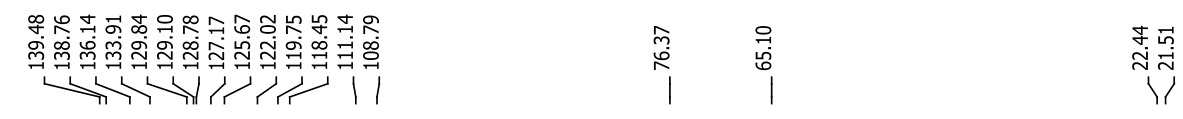

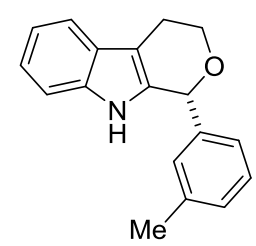

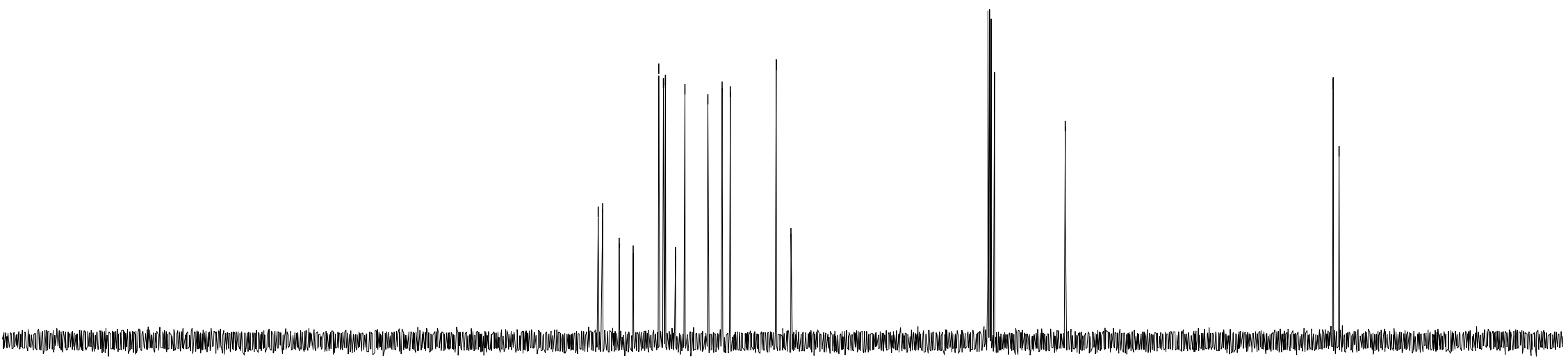

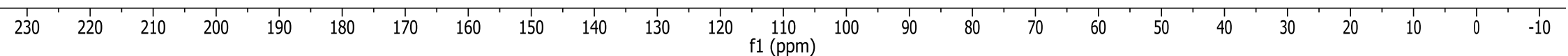




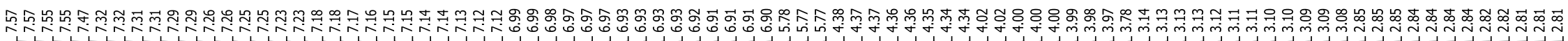

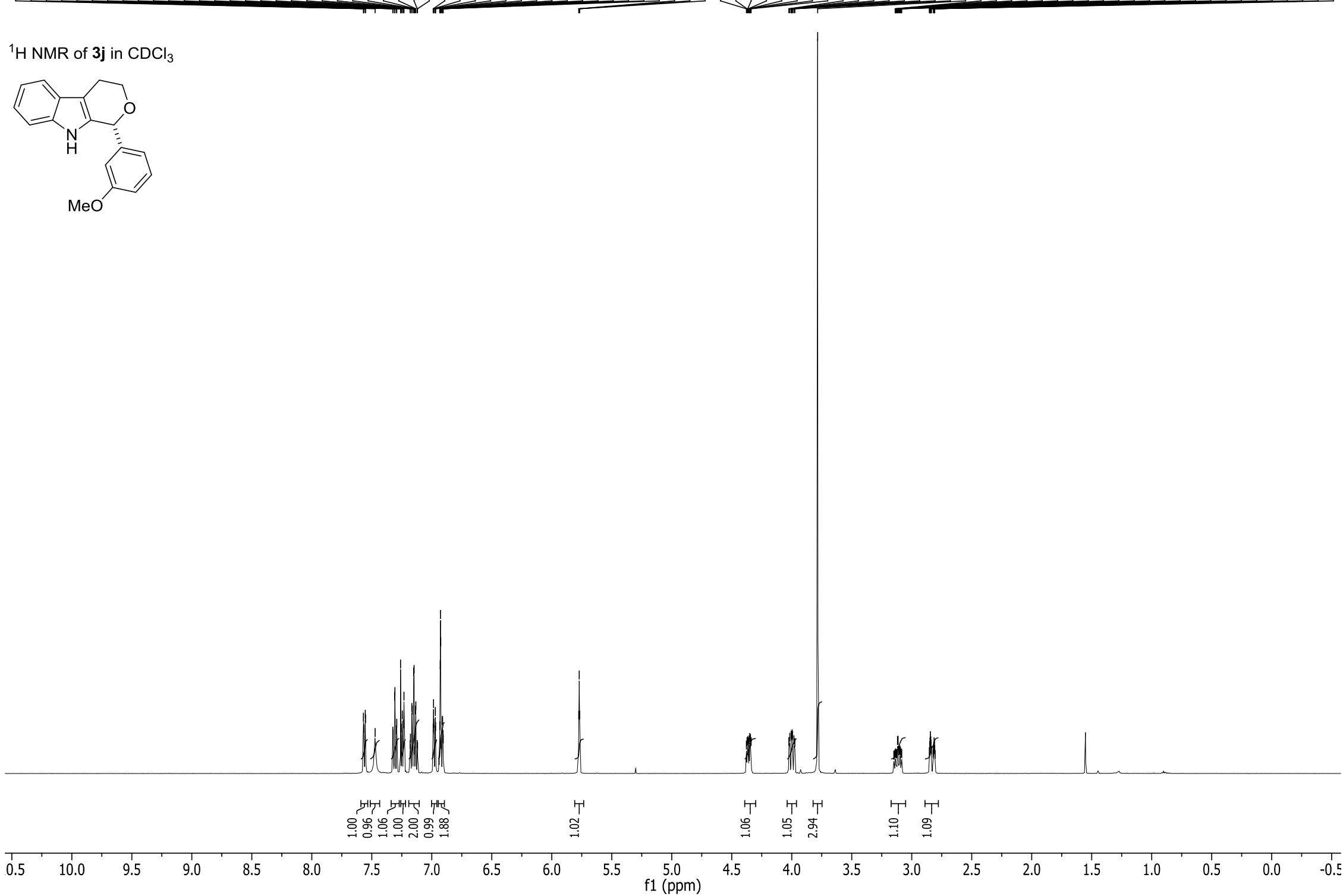


${ }^{13} \mathrm{C} \mathrm{NMR}$ of $3 \mathbf{j}$ in $\mathrm{CDCl}_{3}$

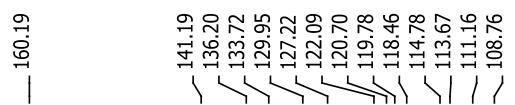

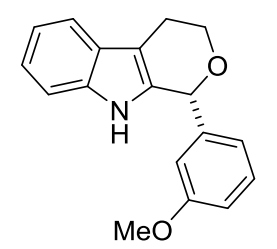

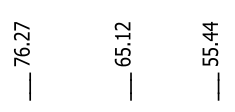

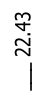

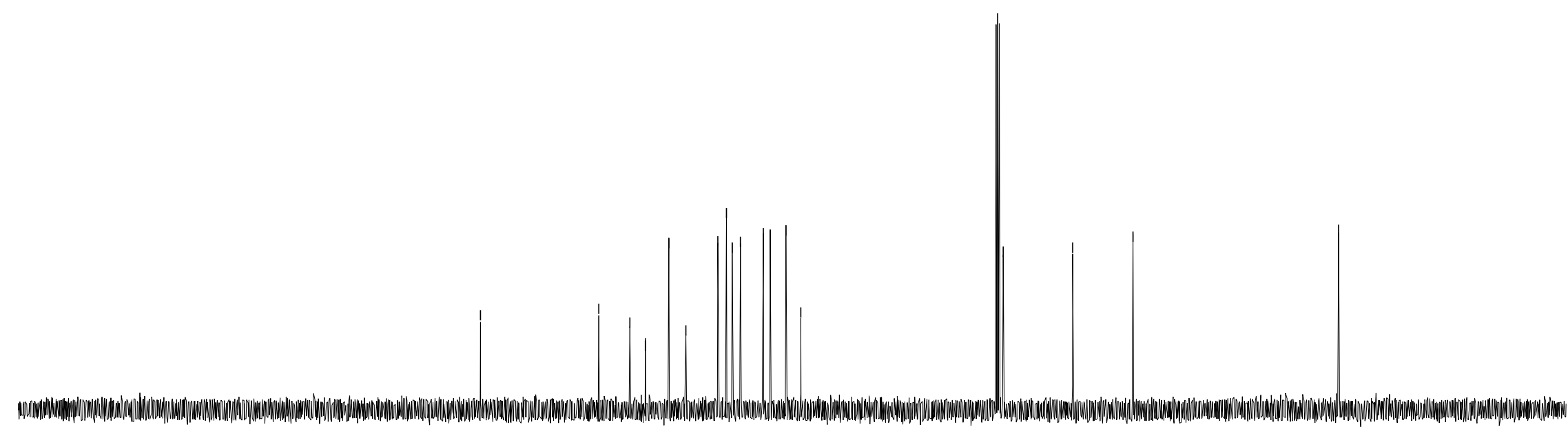

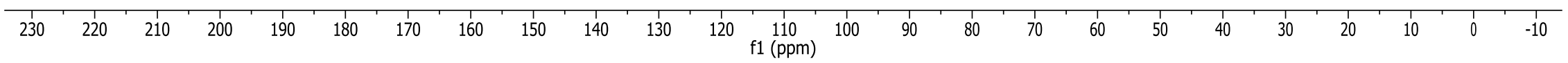


${ }^{1} \mathrm{H}$ NMR of $\mathbf{3 k}$ in $\mathrm{CDCl}_{3}$
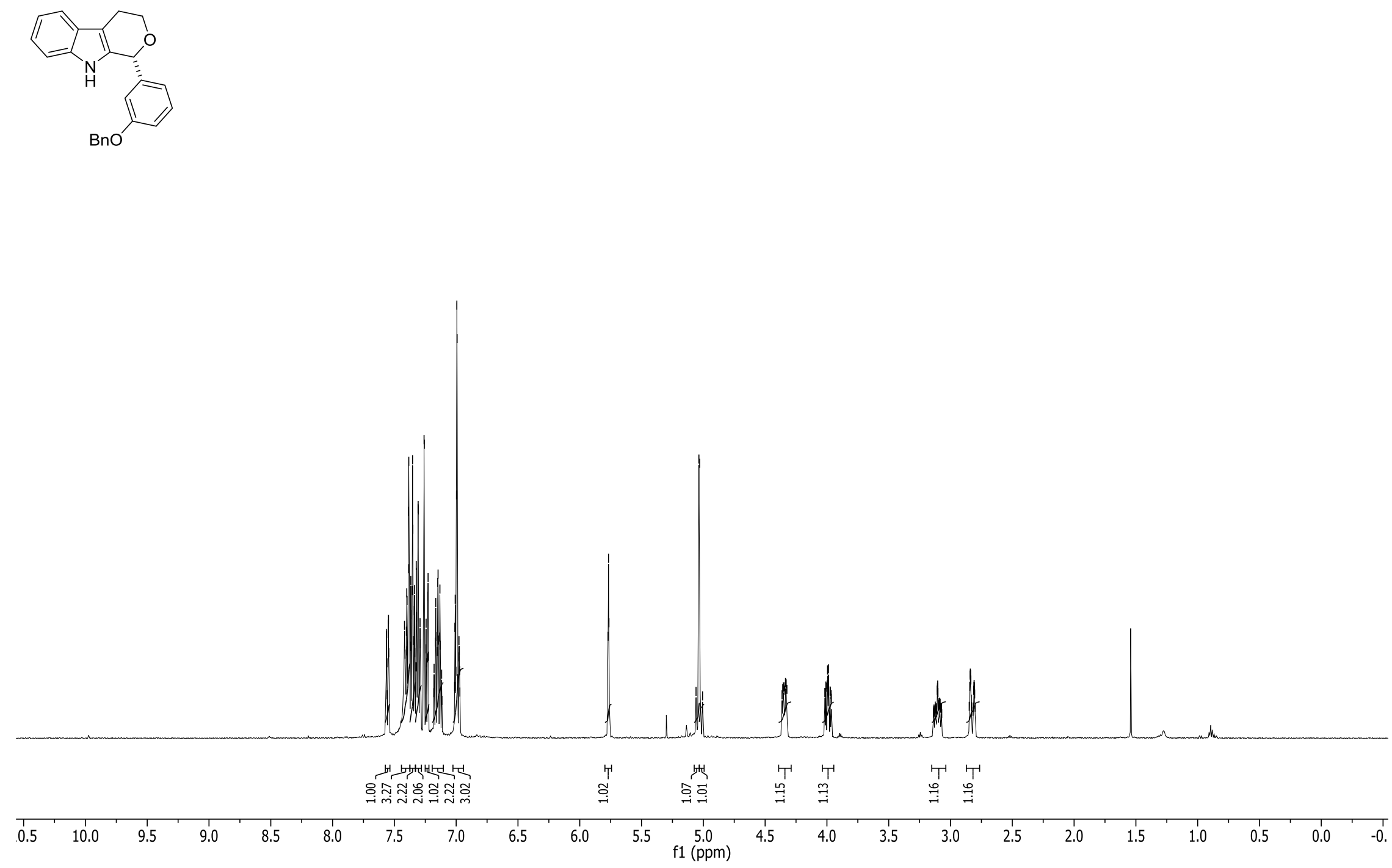
${ }^{13} \mathrm{C} \mathrm{NMR} \mathrm{of} \mathbf{3} \mathbf{k}$ in $\mathrm{CDCl}_{3}$

|

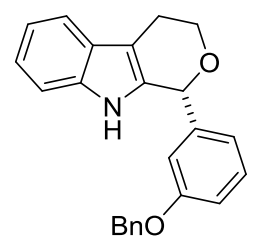

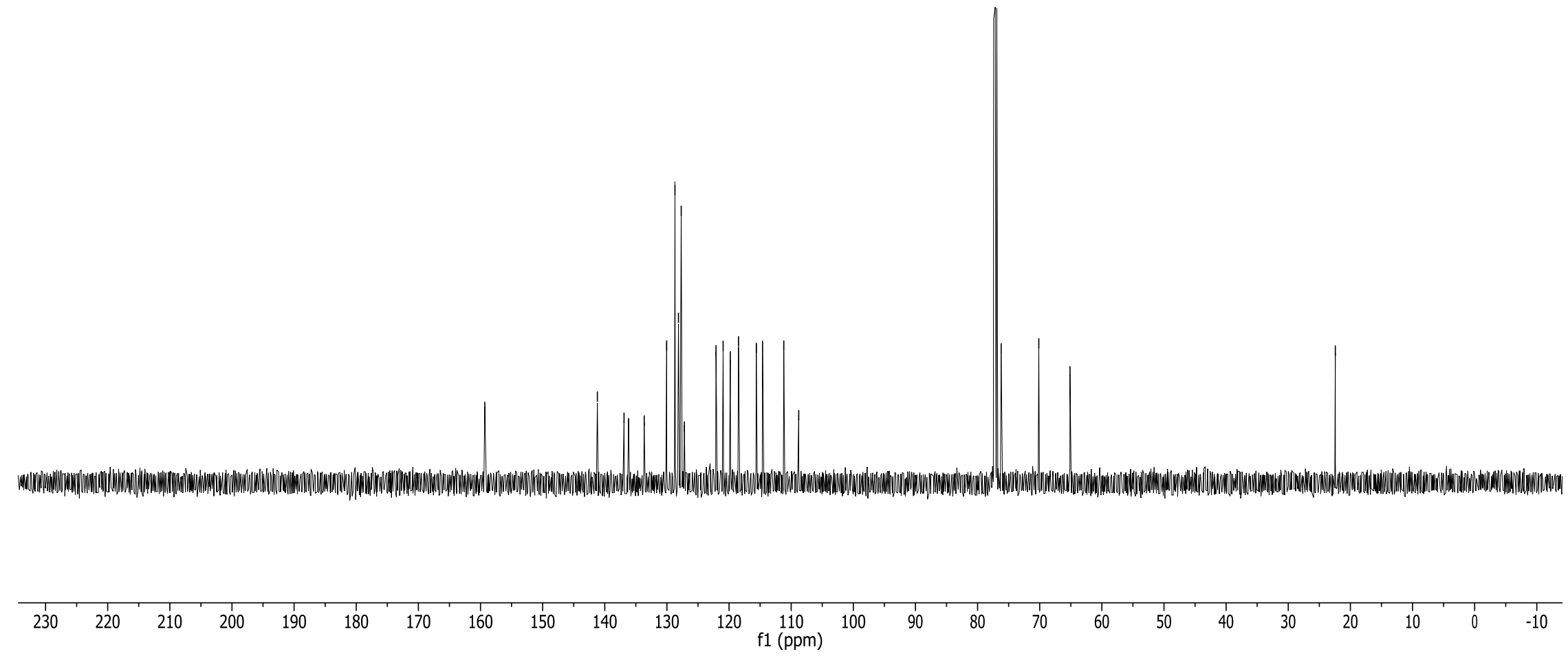




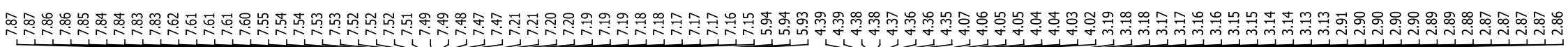
${ }^{1} \mathrm{H} \mathrm{NMR} \mathrm{of} 3 \mathrm{I}$ in $\mathrm{CDCl}_{3}$
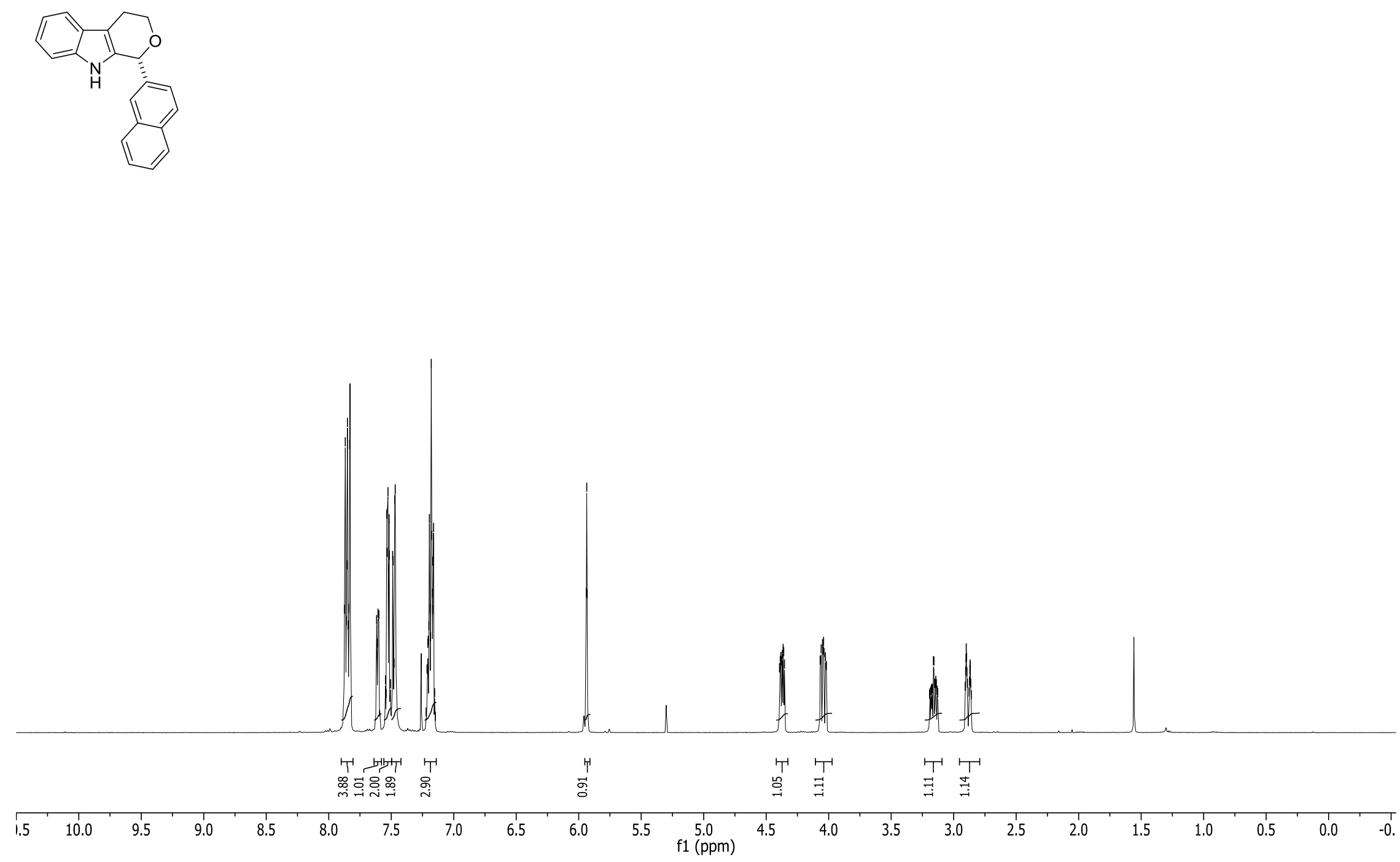
${ }^{13} \mathrm{C} \mathrm{NMR}$ of $\mathbf{3}$ in $\mathrm{CDCl}_{3}$
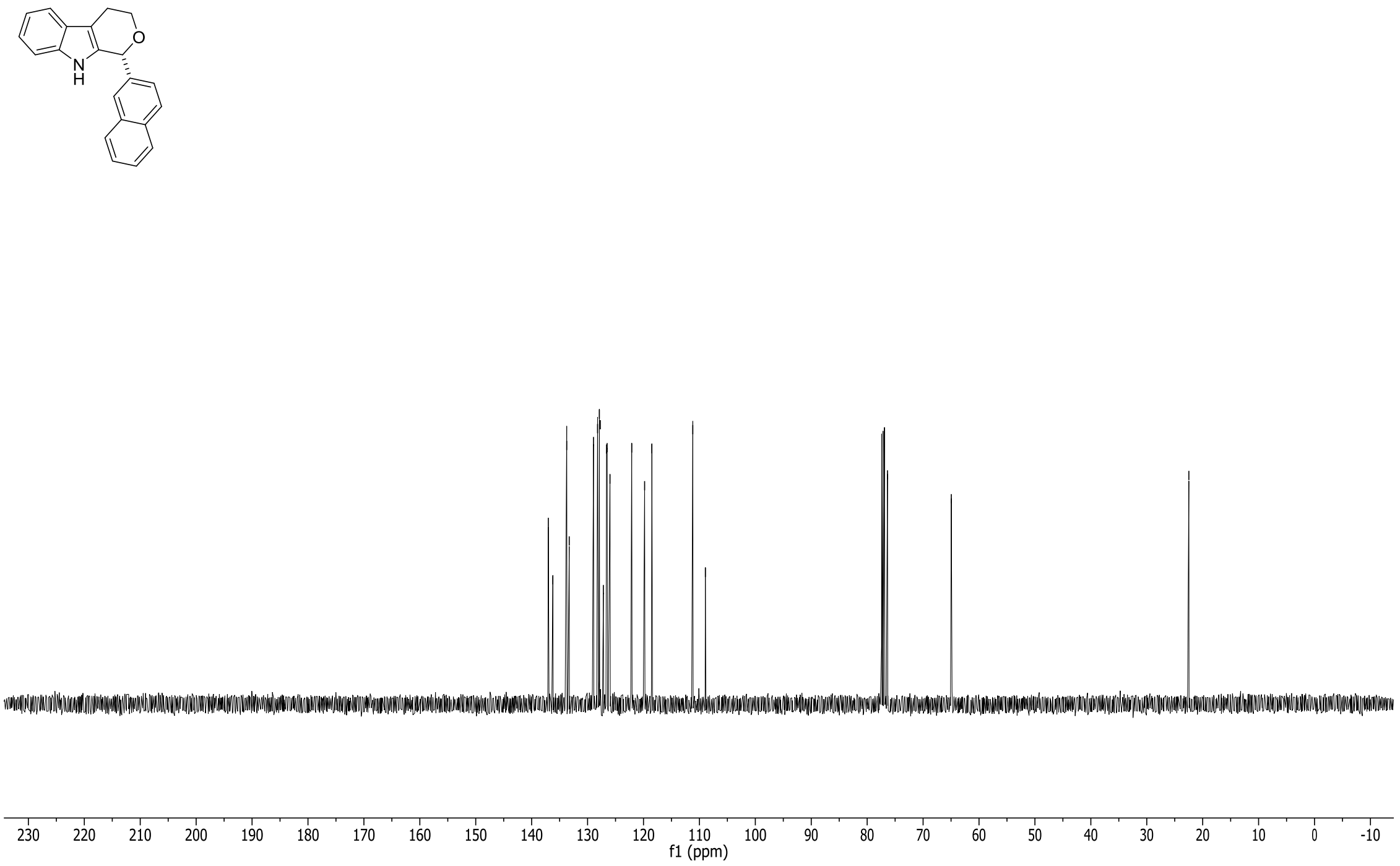


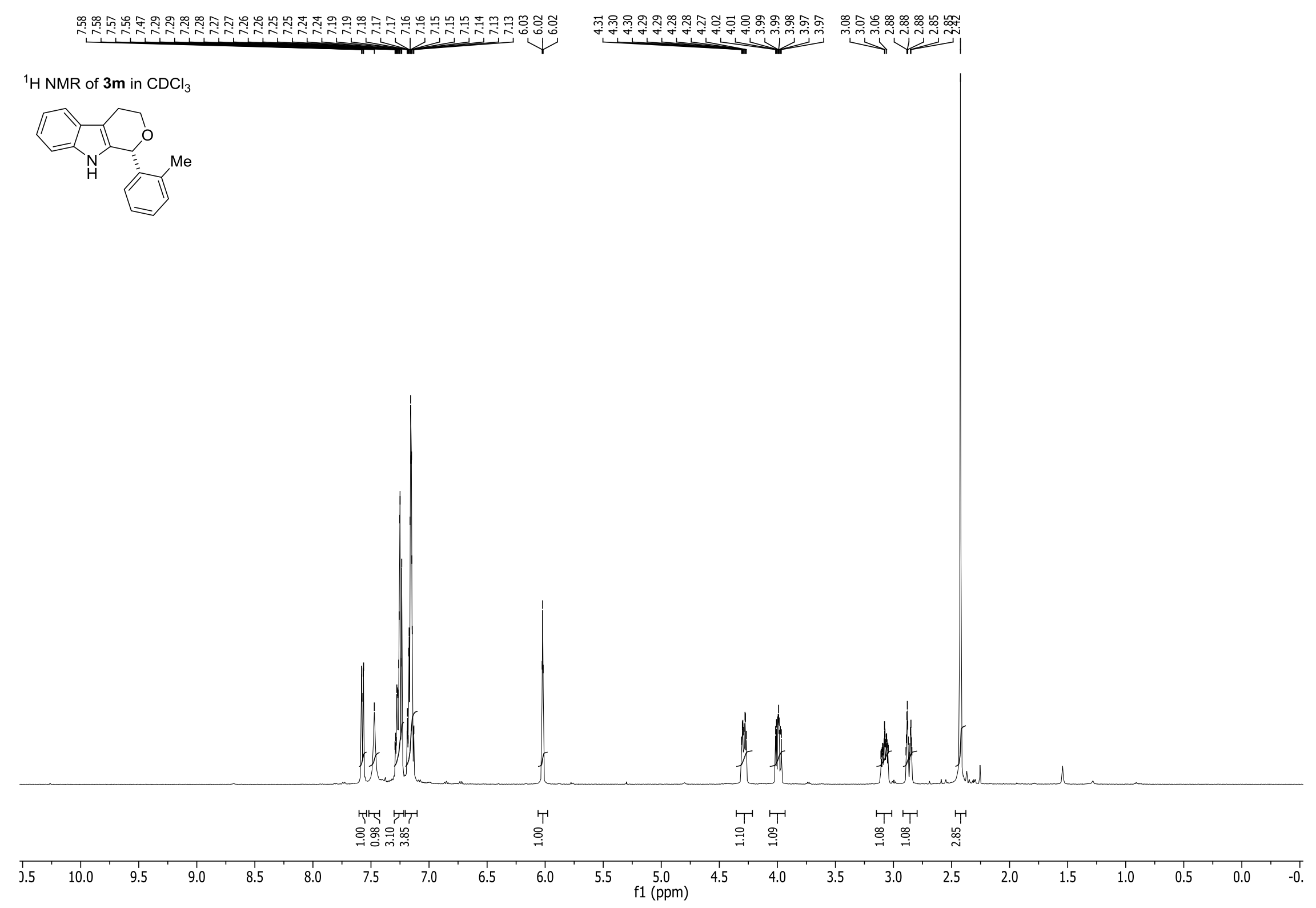




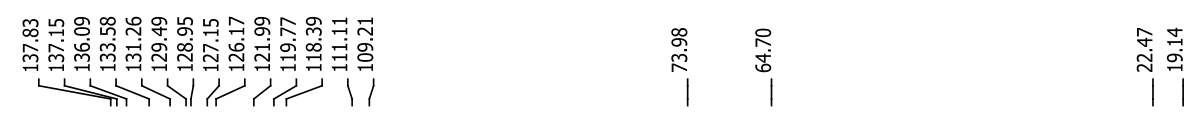

${ }^{13} \mathrm{C} \mathrm{NMR}$ of $3 \mathrm{~m}$ in $\mathrm{CDCl}_{3}$

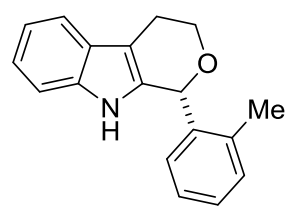

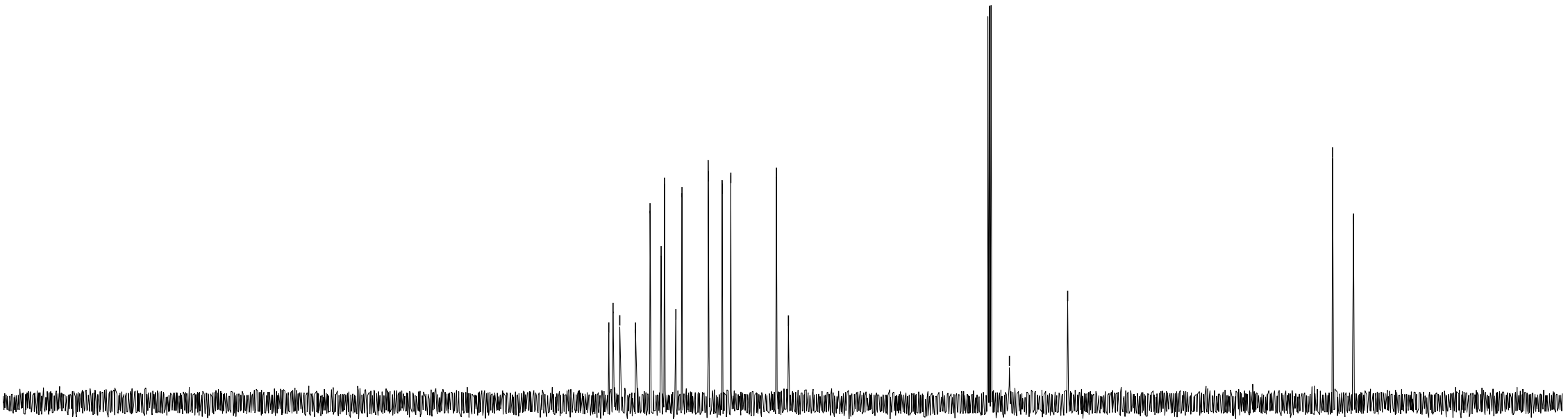

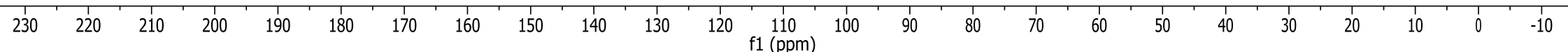




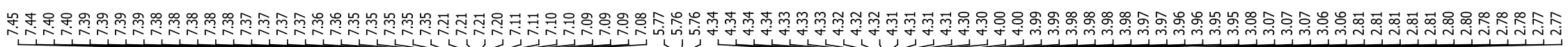
${ }^{1} \mathrm{H} \mathrm{NMR}$ of $3 \mathrm{n}$ in $\mathrm{CDCl}_{3}$

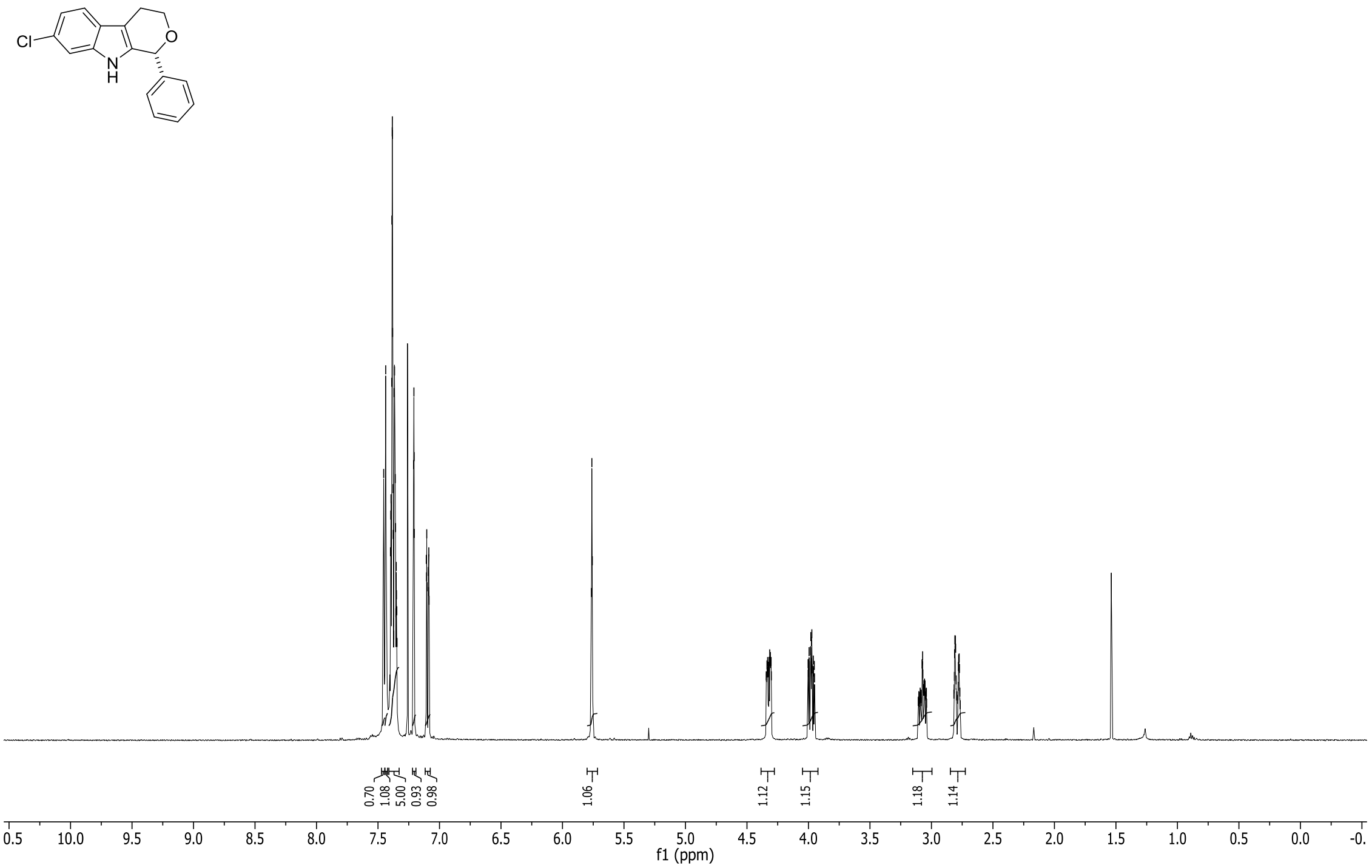




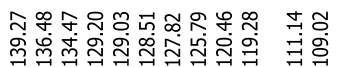

रोपiा।

${ }^{13} \mathrm{C} \mathrm{NMR}$ of $\mathbf{3 n}$ in $\mathrm{CDCl}_{3}$
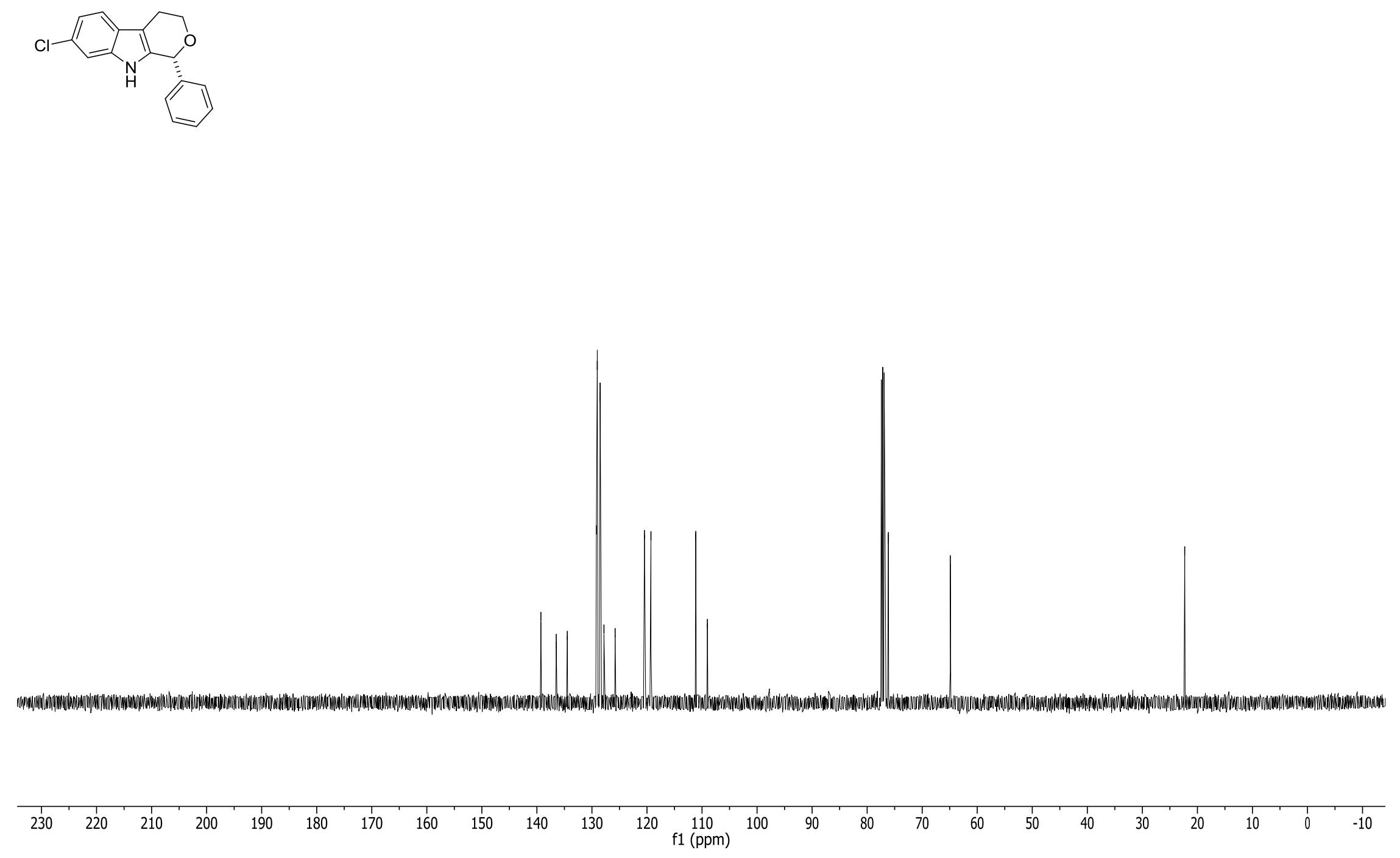


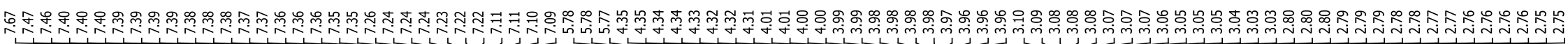
${ }^{1} \mathrm{H} \mathrm{NMR}$ of 30 in $\mathrm{CDCl}_{3}$<smiles>Brc1ccc2[nH]c3c(c2c1)CCO[C@H]3c1ccccc1</smiles>

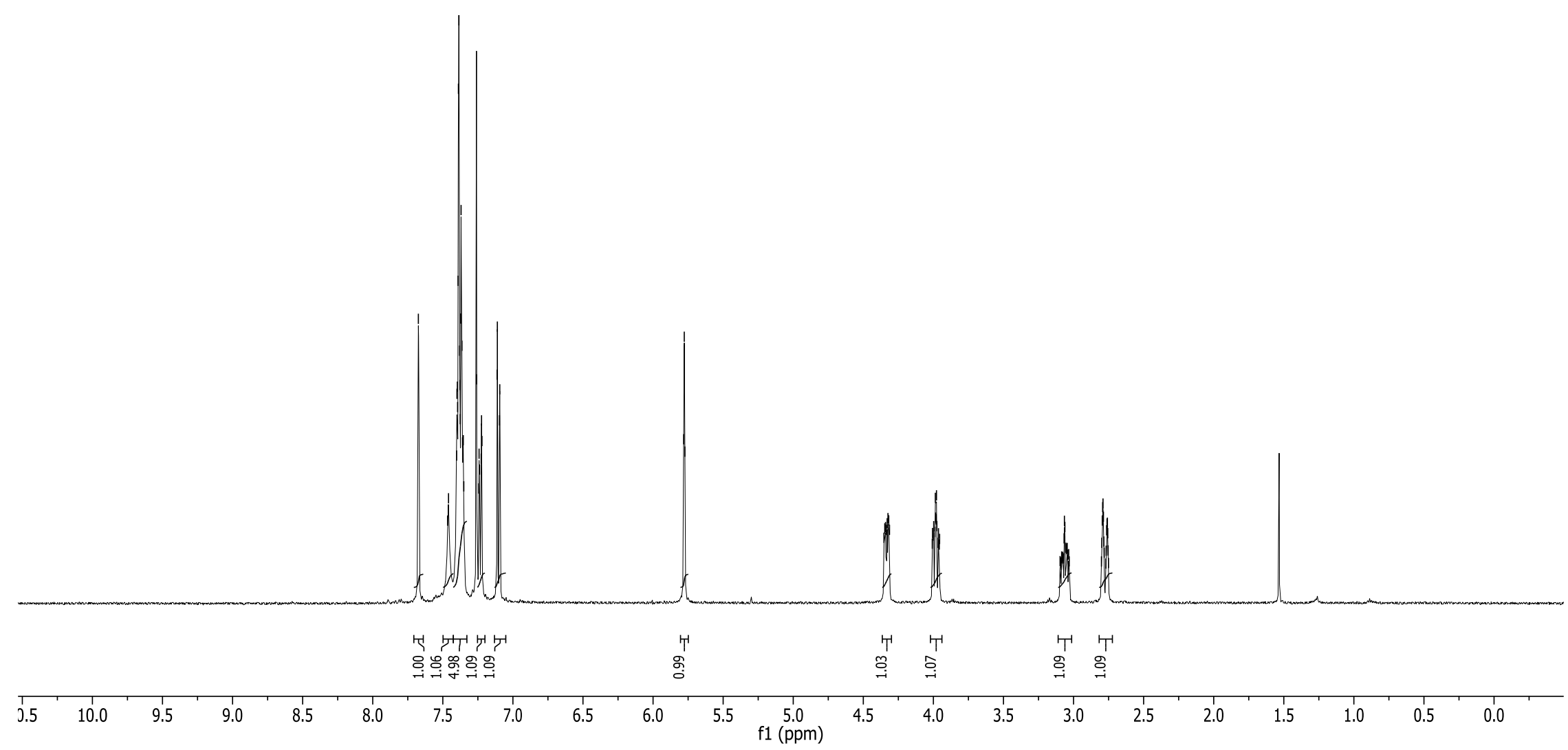


${ }^{13} \mathrm{C} \mathrm{NMR}$ of 30 in $\mathrm{CDCl}_{3}$

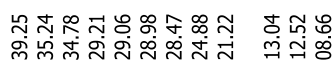

,

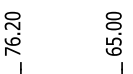

$\stackrel{\text { }}{\text { }}$

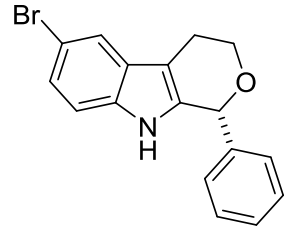

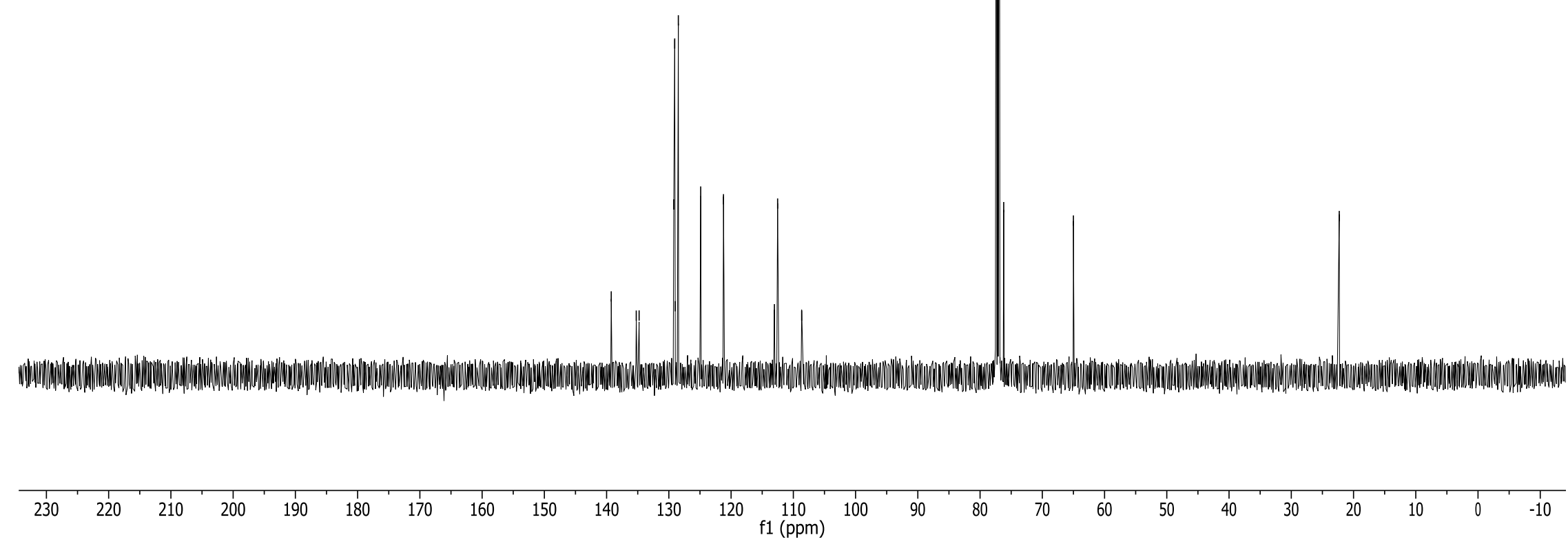




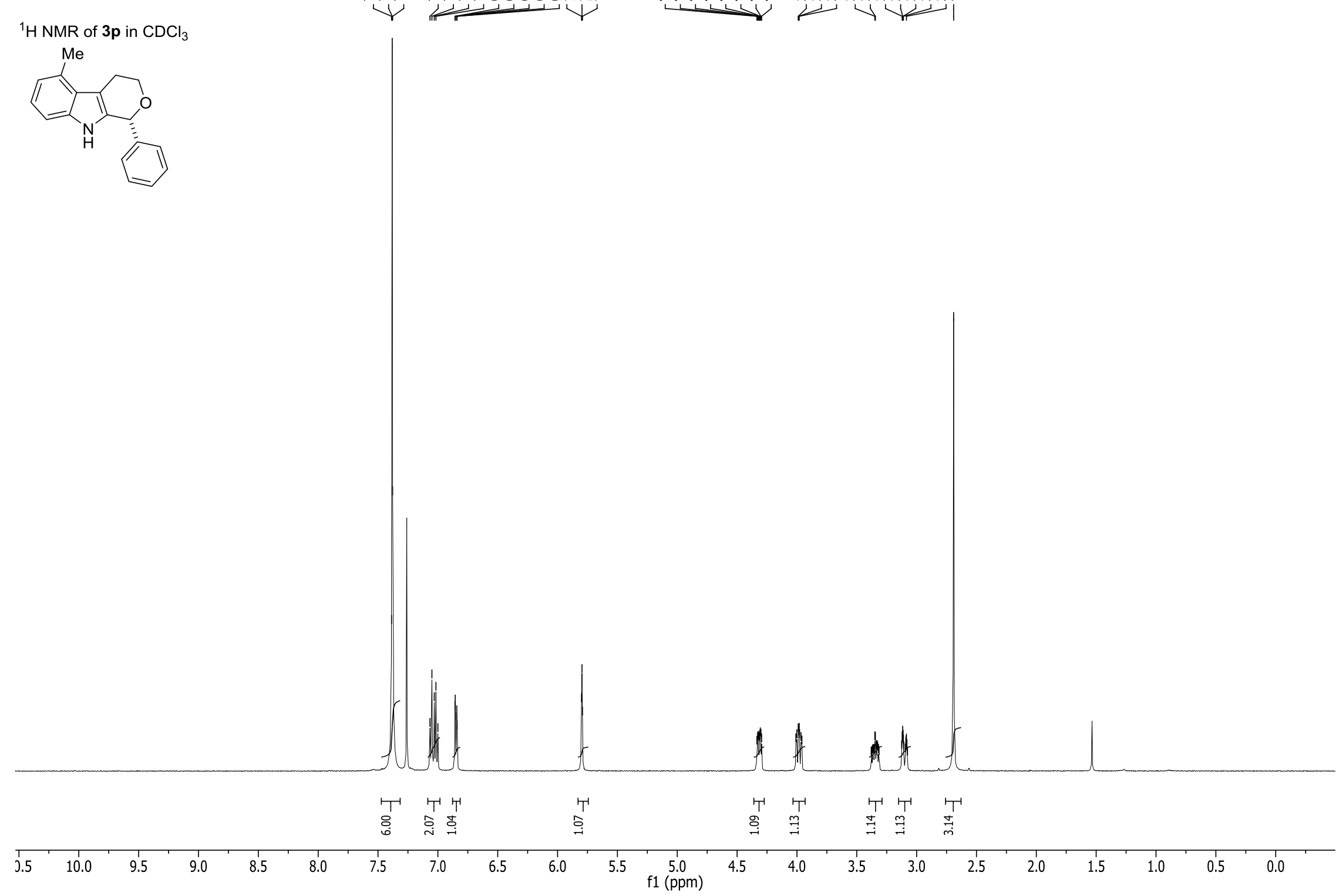




13.

${ }^{13} \mathrm{C}$ NMR of $3 p$ in $\mathrm{CDCl}_{3}$

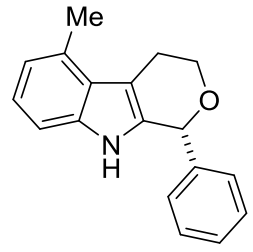

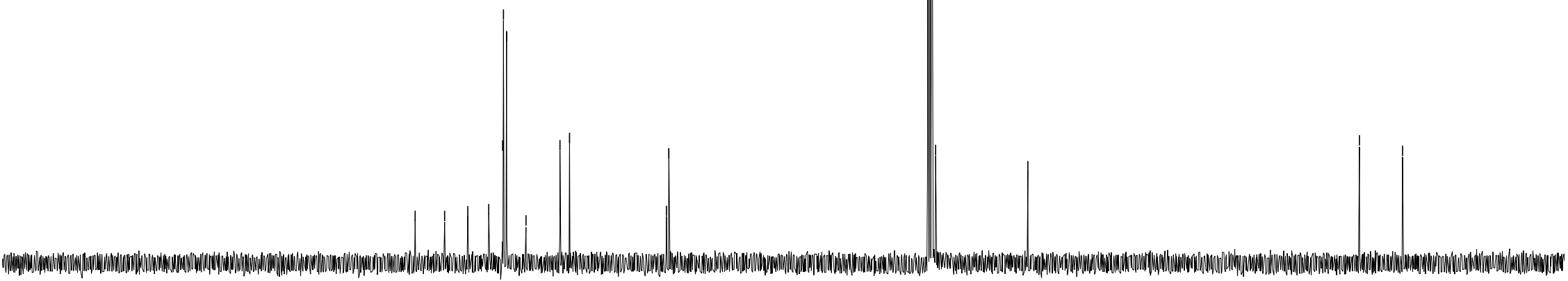

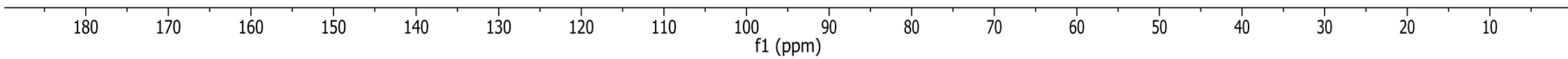


${ }^{1} \mathrm{H}$ NMR of $\mathbf{3 q}$ in $\mathrm{CDCl}_{3}$
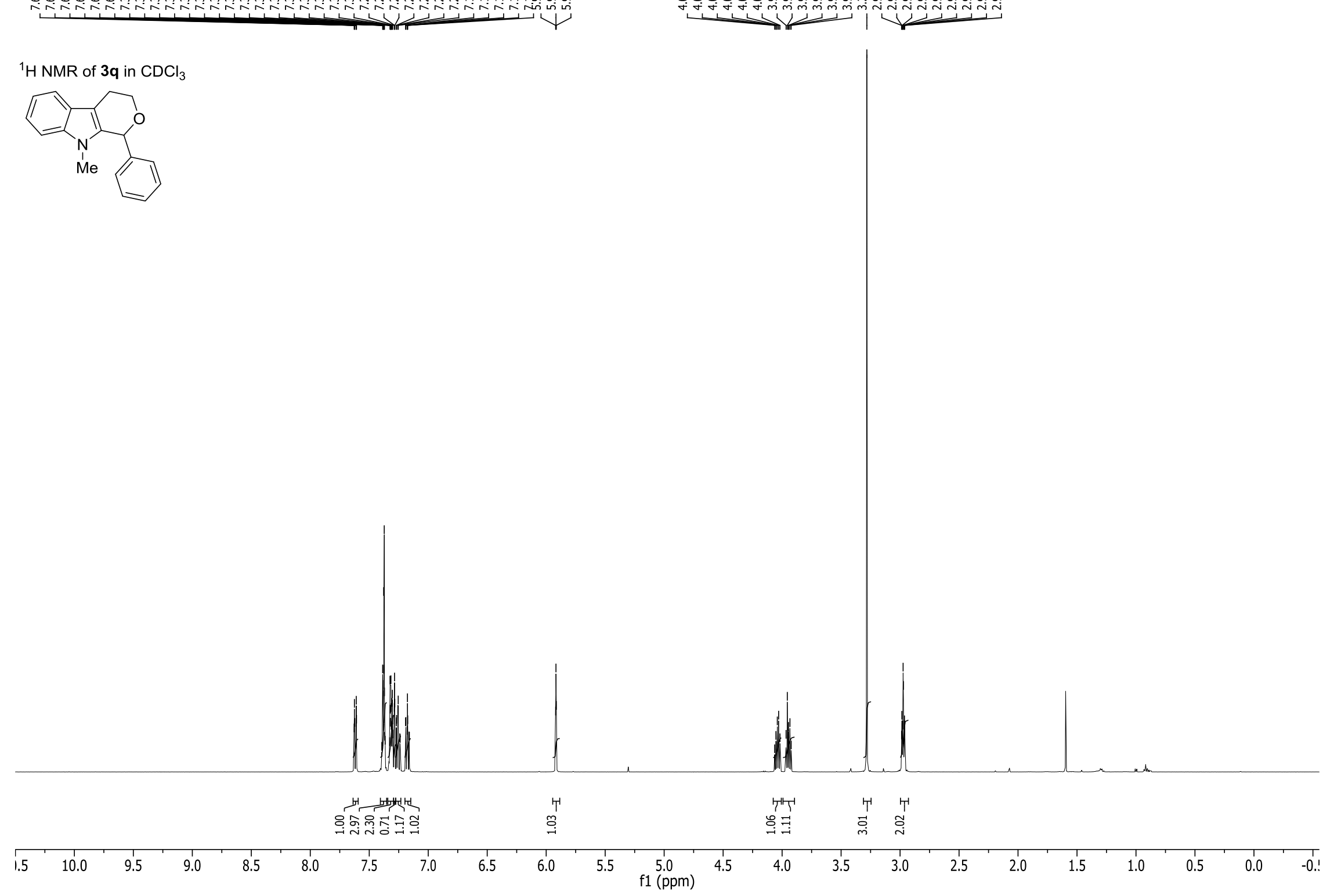
${ }^{13} \mathrm{C} \mathrm{NMR}$ of $\mathbf{3 q}$ in $\mathrm{CDCl}_{3}$

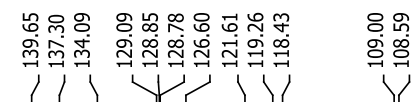

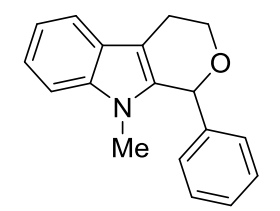

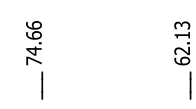

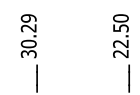

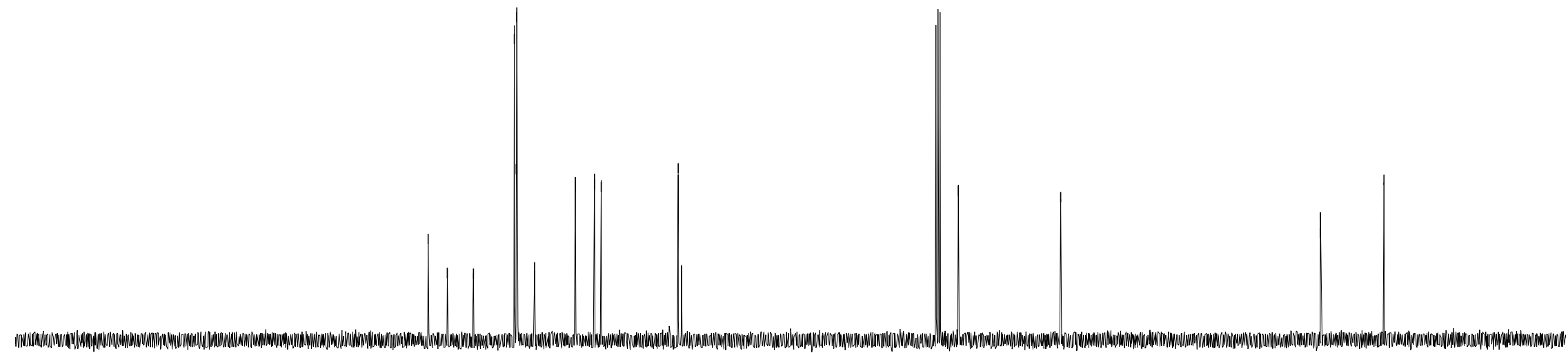

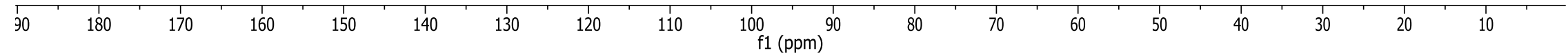



${ }^{1} \mathrm{H} \mathrm{NMR}$ of 4 in $\mathrm{CDCl}_{3}$<smiles>COC(OCCc1c[nH]c2ccccc12)c1ccccc1</smiles>

\section{"ImIII}

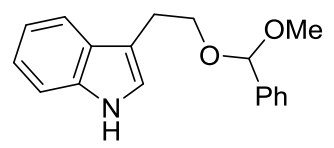

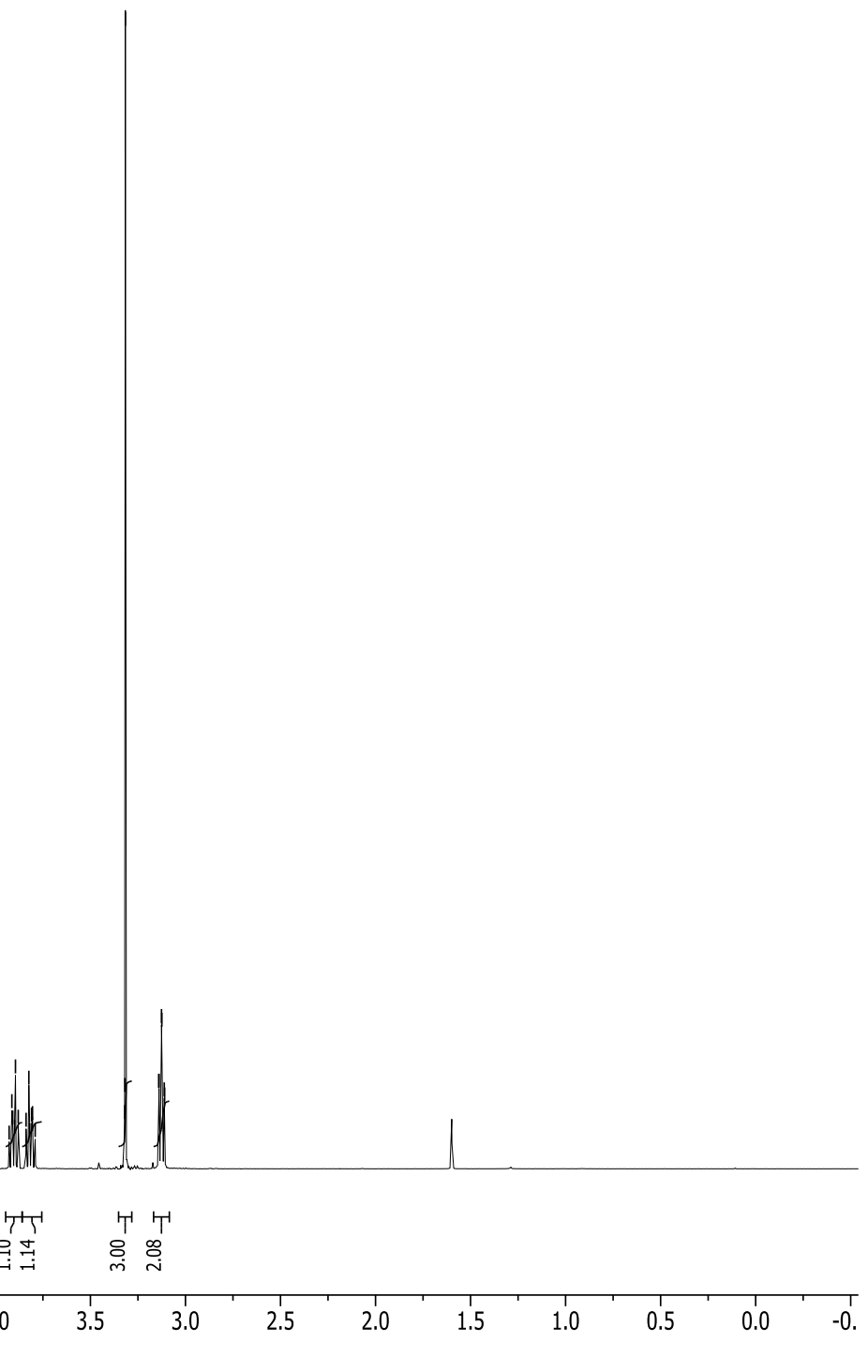




\section{${ }^{13} \mathrm{C}$ NMR of 4 in $\mathrm{CDCl}_{3}$}

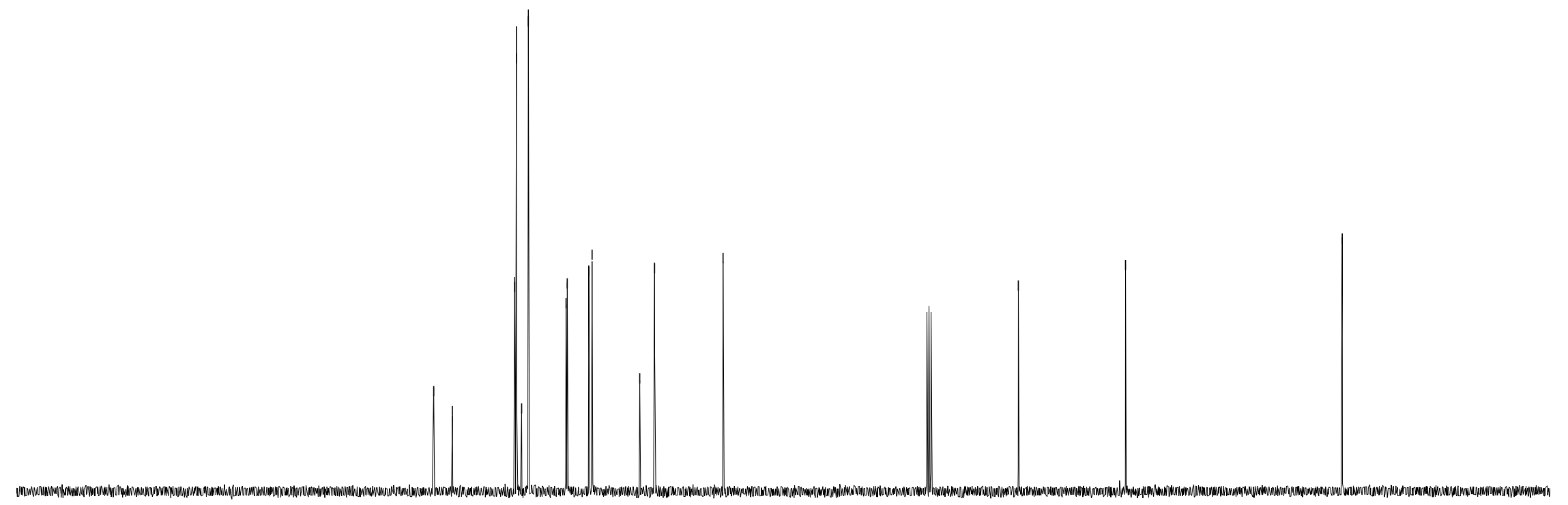

90

180

170




\section{References}

(1) Marigo, M.; Wabnitz, T. C.; Fielenbach, D.; Jørgensen, K. A. Angew. Chem. Int. Ed. 2005, 44, 794.

(2) Alatorre-Santamaría, S.; Rodriguez-Mata, M.; Gotor-Fernández, V.; de Mattos, M. C.; Sayago, F. J.; Jiménez, A. I.; Cativiela, C.; Gotor, V. Tetrahedron: Asymmetry 2008, 19, 1714.

(3) Torisu, K.; Kobayashi, K.; Iwahashi, M.; Nakai, Y.; Onoda, T.; Nagase, T.; Sugimoto, I.; Okada, Y.; Matsumoto, R.; Nanbu, F.; Ohuchida, S.; Nakai, H.; Toda, M. Bioorg. Med. Chem. 2004, 12, 5361.

(4) Sohtome, Y.; Tanatani, A.; Hashimoto, Y.; Nagasawa, K. Tetrahedron Lett. 2004, 45, 5589.

(5) Karagiannidis, L. E.; Hiscock, J. R.; Gale, P. A. Supramol. Chem. 2013, 25, 626.

(6) Kotke, M.; Schreiner, P. R. Tetrahedron 2006, 62, 434.

(7) Tommasino, M. L.; Thomazeau, C.; Touchard, F.; Lemaire, M. Tetrahedron: Asymmetry 1999, 10, 1813.

(8) Gawronski, J.; Kwit, M.; Skowronek, P. Org. Biomol. Chem. 2009, 7, 1562.

(9) Brown, H. C.; McDaniel, D. H. J. Am. Chem. Soc. 1955, 77, 3752.

(10) Chrystiuk, E.; Williams, A. J. Am. Chem. Soc. 1987, 109, 3040.

(11) Collot, A.-G.; Courtney, M.; Coyne, D.; Eustace, S. E.; More O’Ferrall, R. A. J. Org. Chem. 2009, 74, 3356.

(12) Gardner, J. N.; Katritzky, A. R. J. Chem. Soc. 1957, 4375.

(13) Brown, A. R.; Kuo, W.-H.; Jacobsen, E. N. J. Am. Chem. Soc. 2010, 132, 9286.

(14) Tan, K. L.; Jacobsen, E. N. Angew. Chem. Int. Ed. 2007, 46, 1315.

(15) Odabachian, Y.; Wang, Q.; Zhu, J. Chem.-Eur. J. 2013, 19, 12229.

(16) Zhao, C.; Seidel, D. J. Am. Chem. Soc. 2015, 137, 4650. 